\title{
Tropylium-Catalyzed O-H Insertion Reactions of Diazoalkanes with Carboxylic Acids
}

\author{
Supporting Information
}

Claire Empel, ${ }^{\#}$ Thanh Vinh Nguyen* ${ }^{*}$ and Rene M. Koenigs ${ }^{* \#}$

\footnotetext{
\# Institute of Organic Chemistry, RWTH Aachen University, Landoltweg 1, D-52074 Aachen, Germany E-mail: rene.koenigs@rwth-aachen.de

${ }^{\S}$ School of Chemistry, University of New South Wales, Sydney NSW 2052, Australia

E-mail: t.v.nguyen@unsw.edu.au
}

\section{Table of Content}

General Information $\quad$ S1

Important Safety Note $\quad$ S1

$\begin{array}{ll}\text { List of Suppliers } & \text { S1 }\end{array}$

$\begin{array}{ll}\text { Purity of Trop- } \mathrm{BF}_{4} & \mathrm{~S} 2\end{array}$

Reaction Optimization $\quad$ S5

General Procedure $\quad$ S6

1 mmol Scale Experiment $\quad$ S6

$\begin{array}{ll}\text { Preparation of benzoic acid- } d & \text { S7 }\end{array}$

$\begin{array}{ll}\text { Deuterium Labelling Experiments } & \text { S7 }\end{array}$

Control Experiments on Activation Mode for Products 8 and $9 \quad$ S9

$\begin{array}{ll}\text { Chemical Shift Perturbation Experiments } & \text { S10 }\end{array}$

$\begin{array}{ll}\text { Physical Data } & \text { S16 }\end{array}$

$\begin{array}{ll}\text { Reference } & \text { S38 }\end{array}$

$\begin{array}{ll}\text { Spectra } & \text { S39 }\end{array}$ 


\section{General Information}

Unless otherwise noted, all commercially available compounds were used as provided without further purification. Tropylium tetrafluoroborate was purchased from TCI, for the supplier of carboxylic acids, can be found in the list of suppliers. Solvents used in reactions were p.A. grade. Solvents for chromatography were technical grade and distilled prior to use. Analytical thin-layer chromatography (TLC) was performed on Macherey-Nagel silica gel aluminium plates with F-254 indicator, visualized by irradiation with UV light. Column chromatography was performed using silica gel Merck 60 (particle size $0.063-0.2 \mathrm{~mm}$ ). Solvent mixtures are understood as volume/volume. ${ }^{1} \mathrm{H}$ NMR, ${ }^{13} \mathrm{C}$ NMR and ${ }^{19} \mathrm{~F}$ NMR were recorded on a Varian AV600/AV400 or an Agilent DD2 $400 \mathrm{NMR}$ spectrometer in $\mathrm{CDCl}_{3}$. Data are reported in the following order: chemical shift $(\delta)$ in ppm; multiplicities are indicated br (broadened singlet), $\mathrm{s}$ (singlet), d (doublet), t (triplet), q (quartet), m (multiplet); coupling constants $(J)$ are in Hertz $(\mathrm{Hz})$. HRMS data were recorded on a ThermoFisher Scientific LTQ Orbitrap XL using ESI ionization or on a Finnigan MAT 95 using EI ionization at $70 \mathrm{eV}$. IR spectra were recorded on a Perkin Elmer 100 spectrometer and are reported in terms of frequency of absorption $\left(\mathrm{cm}^{-1}\right)$.

\section{Important Safety Note}

Handling of diazo compounds should only be done in a well-ventilated fume cupboard using an additional blast shield. No incidents occurred handling of diazoalkanes during the preparation of this manuscript, yet the reader should be aware of carcinogenicity and explosiveness of the herein described diazo compounds. General safety precautions when working with diazomethane and its derivatives should be followed. Any reactions described in this manuscript should not be performed without strict risk assessment and proper safety precautions.

\section{List of Suppliers for Carboxylic Acids}

\begin{tabular}{ll}
\hline Supplier & Acids or Acids for Product Synthesis \\
\hline Acros & $6 \mathrm{~d}, 6 \mathrm{e}, 7 \mathrm{q}, 7 \mathrm{v}, 12 \mathrm{~d}, 12 \mathrm{i}, 12 \mathrm{j}$ \\
Alfer Aeser & $6 \mathrm{~b}, 7 \mathrm{aa}, 12 \mathrm{f}, 12 \mathrm{~h}, 12 \mathrm{l}, 12 \mathrm{~m}$ \\
Chempure & $7 \mathrm{z}$ \\
Fluorochem & $6 \mathrm{f}, 7 \mathrm{r}, 7 \mathrm{w}, 12 \mathrm{k}$ \\
Grüssing & $12 \mathrm{~b}$ \\
Merck & $7 \mathrm{q}, 7 \mathrm{u}, 12 \mathrm{a}, 12 \mathrm{c}, 12 \mathrm{~g}$, \\
Sigma Aldrich & $6 \mathrm{a}, 6 \mathrm{c}, 7 \mathrm{a}, 7 \mathrm{n}, 7 \mathrm{p}, 7 \mathrm{~s}, 7 \mathrm{t}, 7 \mathrm{x}, 7 \mathrm{y}, 11$ \\
\hline
\end{tabular}




\section{Purity of Trop-BF 4}

Trop- $\mathrm{BF}_{4}$ is a hydroscopic compound and needs to be carefully stored under exclusion of air and moisture at $2-8{ }^{\circ} \mathrm{C}$. The purity of Trop- $\mathrm{BF}_{4} 19$ was analysed by ${ }^{1} \mathrm{H}$ and ${ }^{19} \mathrm{~F}$ NMR spectroscopy in acetonitrile- $d_{3}$.

In the ${ }^{19} \mathrm{~F}$ NMR of Trop- $\mathrm{BF}_{4} \mathbf{1 9}$ only the $\mathrm{BF}_{4}-$ species is visible.

${ }^{19}$ F NMR (376 MHz, Acetonitrile- $\left.d_{3}\right)$

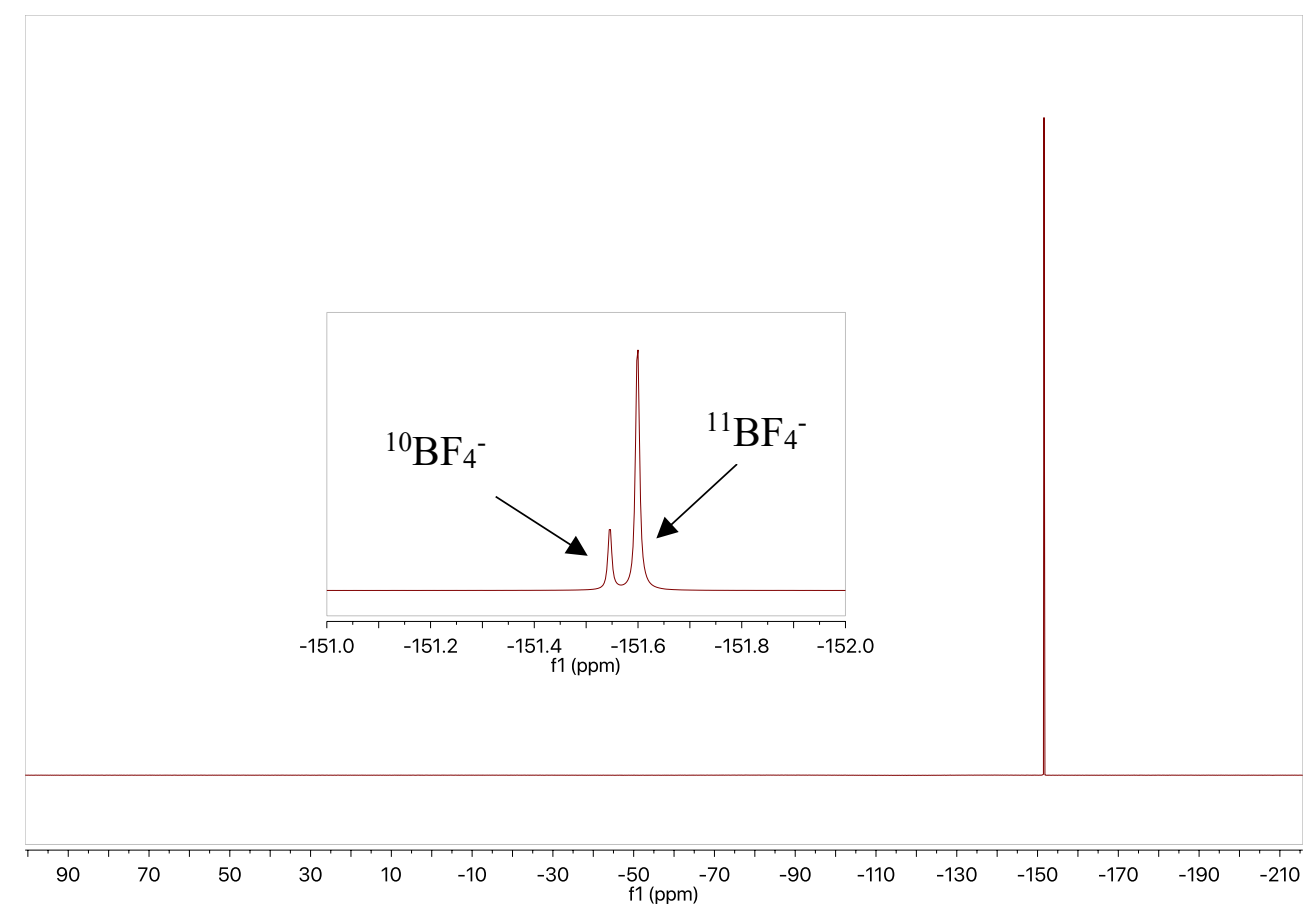

In additional experiments, we added 1, 2, 5 and 10 eq. of water, respectively and observed a shift of the signals for $\mathrm{BF}_{4}^{-}$using hexafluorobenzene as an internal standard.

${ }^{19}$ F NMR (376 MHz, Acetonitrile- $d_{3}$ )

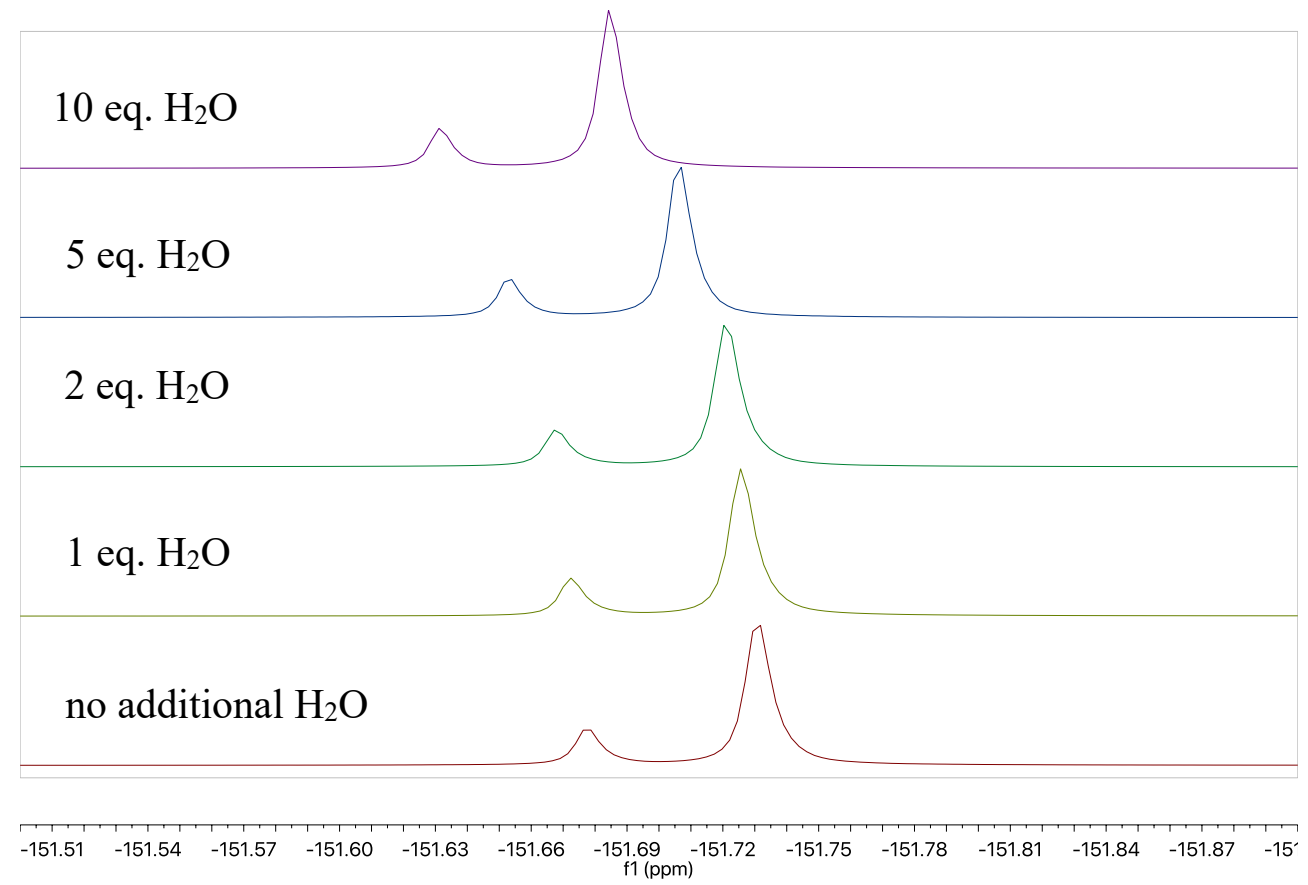


In further 19F NMR studies we compared an aq. solution of $\mathrm{HBF}_{4}$ with the $\mathrm{BF}_{4}$ species present in Trop- $\mathrm{BF}_{4}$ 19. A significant chemical shift was observed, which indicates that $\mathrm{HBF}_{4}$ is not present in Trop-BF 4 19. For details on $N M R$ of aq. solution of $\mathrm{HBF}_{4}$, please see reference 1.

${ }^{19}$ F NMR (376 MHz, Acetonitrile- $\left.d_{3}\right)$

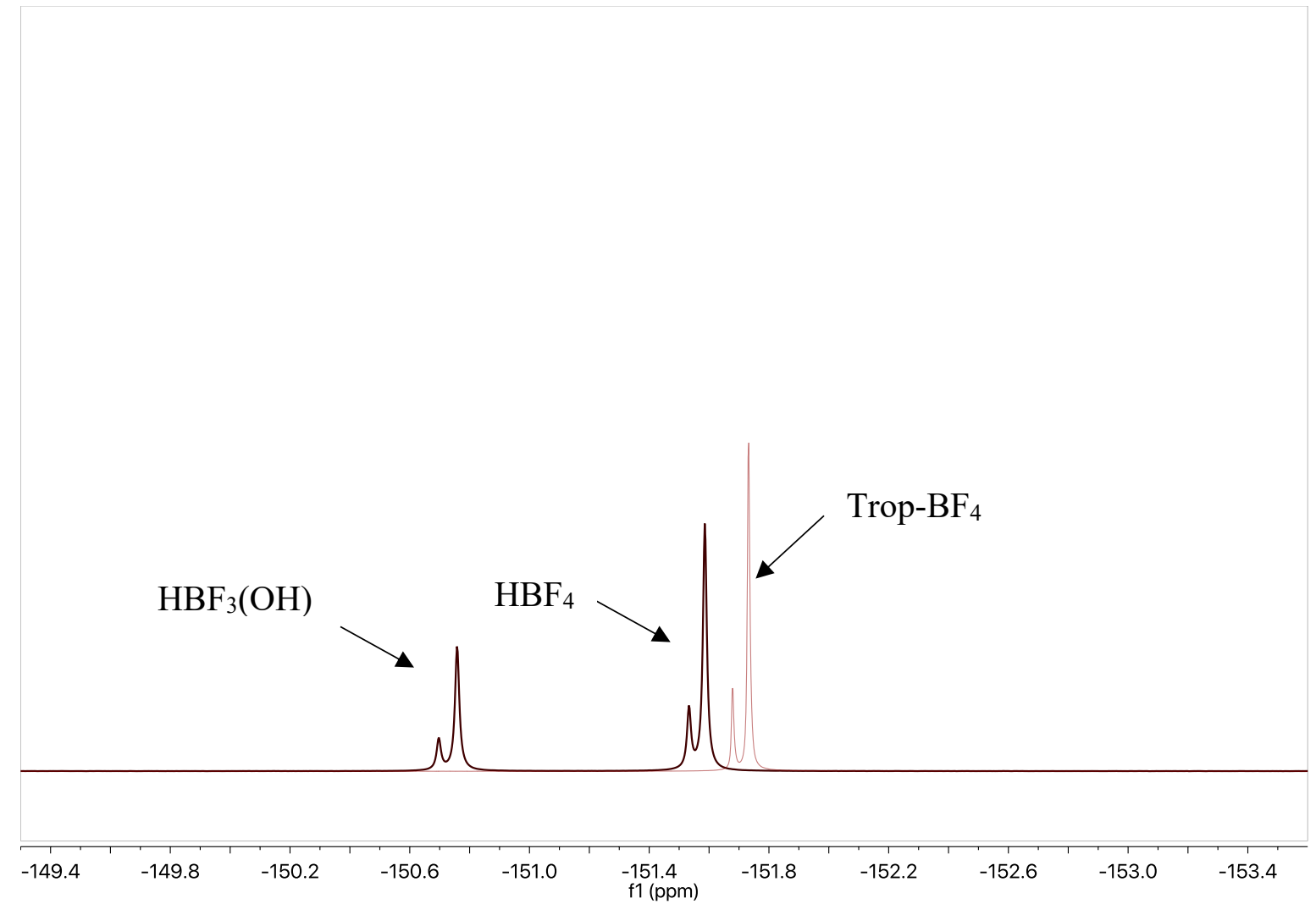

\section{Chemical Shift of Different Species}

\begin{tabular}{lll}
\hline Species & $\delta\left(\mathrm{F}^{10} \mathrm{~B}\right) \mathrm{ppm}$ & $\delta\left(\mathrm{F}^{11} \mathrm{~B}\right) \mathrm{ppm}$ \\
\hline $\mathrm{HBF}_{3}(\mathrm{OH})$ & -150.70 & -150.76 \\
$\mathrm{HBF}_{4}$ & -151.53 & -151.59 \\
$\mathrm{Trop}_{-} \mathrm{BF}$ & -151.68 & -151.73 \\
\hline
\end{tabular}


Amount of water in acetonitrile- $d_{3}$ was determined by mesitylene $(0.1 \mathrm{M})$ as internal standard. ${ }^{1}$ H NMR (400 MHz, Acetonitrile- $d_{3}$ )
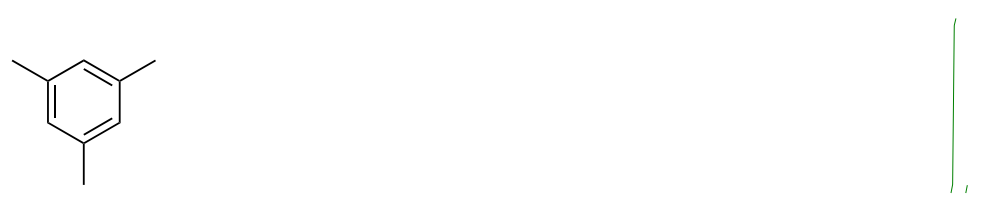

$c=0.1 \mathrm{M}$

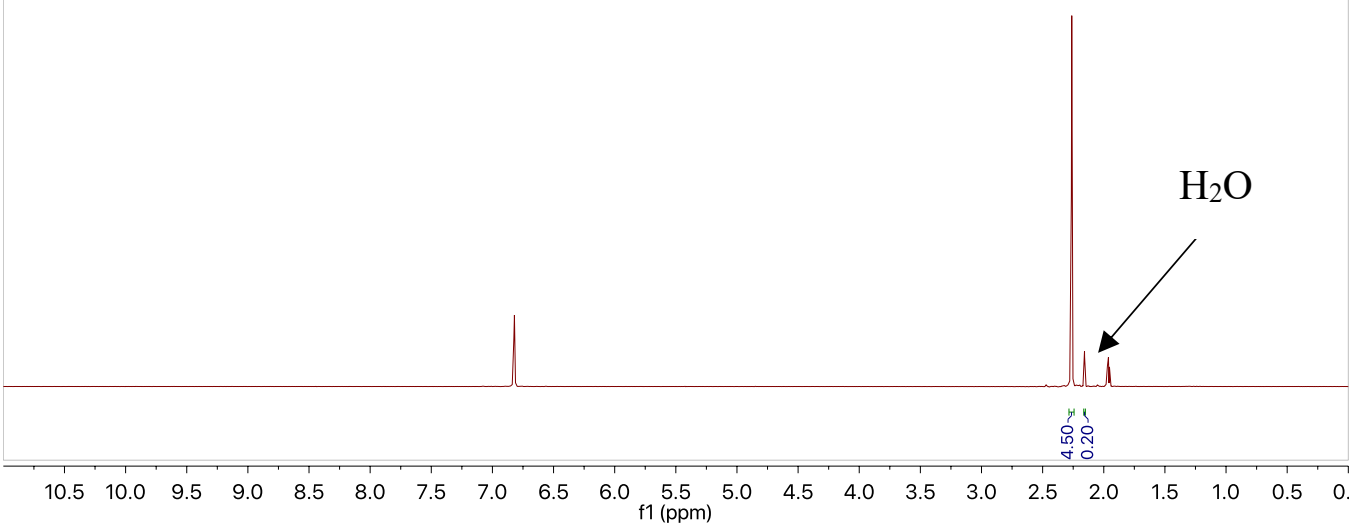

The amount water in Trop-BF $419(c=0.1 \mathrm{M})$ was determined using mesitylene $(c=0.1 \mathrm{M})$ as an internal standard. The amount of water was calculated to be $\sim 5 \%$ according to ${ }^{1} \mathrm{H}$ NMR spectroscopy.

${ }^{1}$ H NMR (400 MHz, Acetonitrile- $d_{3}$ )

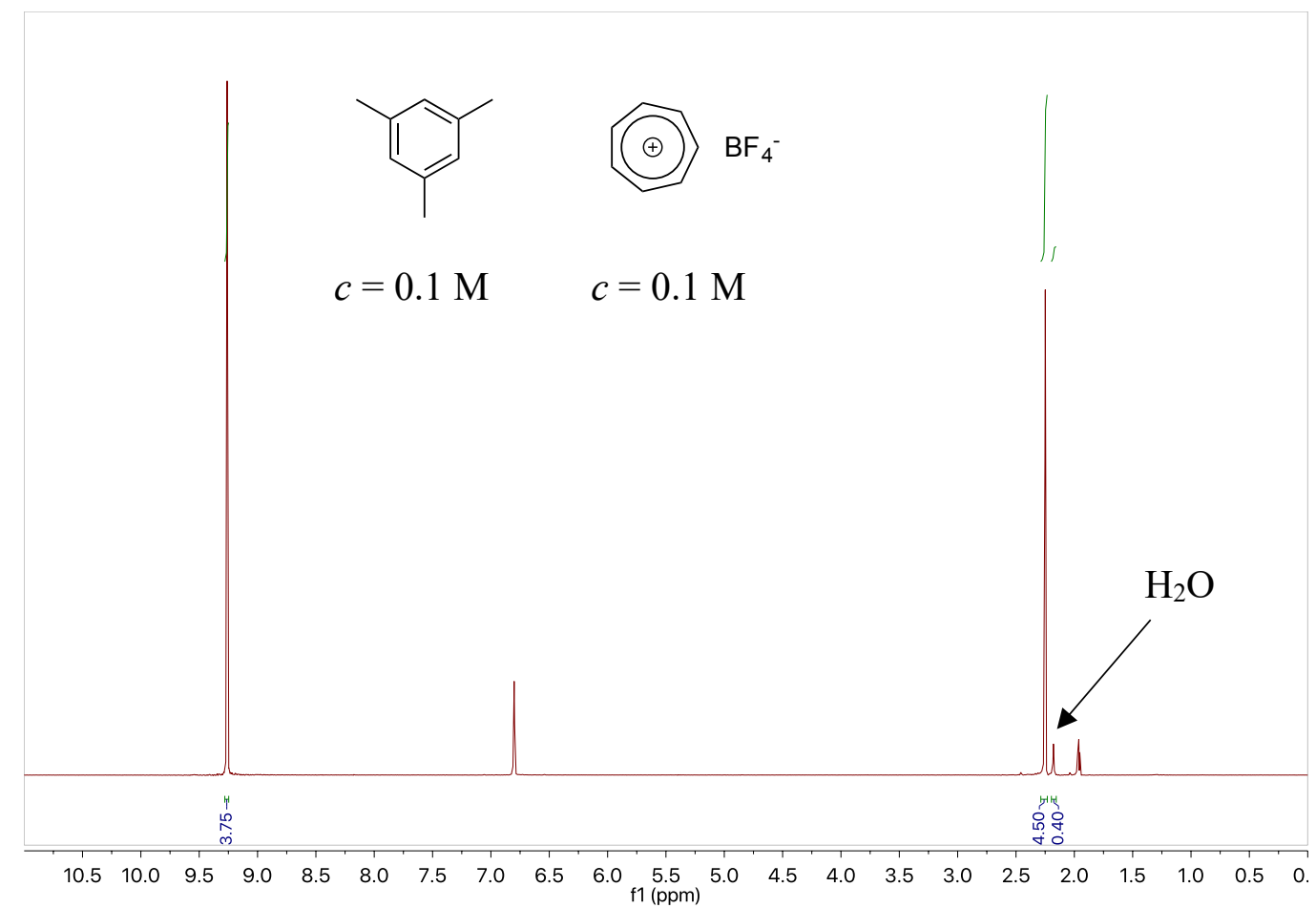




\section{Reaction Optimization}<smiles>COC(=O)C(=N)c1ccccc1</smiles>

1a<smiles>O=C(O)c1ccccc1</smiles>

$6 a$ reaction conditions<smiles>COC(=O)C(OC(=O)c1ccccc1)c1ccccc1</smiles>

\begin{tabular}{|c|c|c|c|c|}
\hline Entry $^{[a]}$ & Catalyst & Solvent & Equivalents 1a : 6a & Yield $^{[b]}$ \\
\hline 1 & $\operatorname{Trop}\left(\mathrm{BF}_{4}\right)(5 \mathrm{~mol} \%)$ & THF & $1: 1.2$ & $29 \%$ \\
\hline 2 & $\operatorname{Trop}\left(\mathrm{BF}_{4}\right)(5 \mathrm{~mol} \%)$ & $\mathrm{MeCN}$ & $1: 1.2$ & no rct. \\
\hline 3 & $\operatorname{Trop}\left(\mathrm{BF}_{4}\right)(5 \mathrm{~mol} \%)$ & acetone & $1: 1.2$ & no rct. \\
\hline 4 & $\operatorname{Trop}\left(\mathrm{BF}_{4}\right)(5 \mathrm{~mol} \%)$ & toluene & $1: 1.2$ & no rct. \\
\hline 5 & $\operatorname{Trop}\left(\mathrm{BF}_{4}\right)(5 \mathrm{~mol} \%)$ & $\mathrm{MeOH}$ & $1: 1.2$ & no rct. \\
\hline 6 & $\operatorname{Trop}\left(\mathrm{BF}_{4}\right)(5 \mathrm{~mol} \%)$ & 1,2-DCE & $1: 1.2$ & $44 \%$ \\
\hline 7 & $\operatorname{Trop}\left(\mathrm{BF}_{4}\right)(5 \mathrm{~mol} \%)$ & DCM & $1: 1.2$ & $70 \%$ \\
\hline 8 & $\operatorname{Trop}\left(\mathrm{BF}_{4}\right)(5 \mathrm{~mol} \%)$ & DCM & $1: 2$ & $78 \%$ \\
\hline 9 & $\operatorname{Trop}\left(\mathrm{BF}_{4}\right)(5 \mathrm{~mol} \%)$ & DCM & $2: 1$ & $65 \%$ \\
\hline 10 & Trop $\left(\mathrm{BF}_{4}\right)(10 \mathrm{~mol} \%)$ & DCM & $1: 1.2$ & $92 \%$ \\
\hline 11 & Trop $\left(\mathrm{BF}_{4}\right)(2 \mathrm{~mol} \%)$ & DCM & $1: 1.2$ & $68 \%$ \\
\hline 12 & $\operatorname{Trop}\left(\mathrm{BF}_{4}\right)(1 \mathrm{~mol} \%)$ & DCM & $1: 1.2$ & $64 \%$ \\
\hline 13 & $\operatorname{Trop}\left(\mathrm{BF}_{4}\right)(5 \mathrm{~mol} \%)$ & DCM & no benzoic acid & decomp. \\
\hline 14 & - & DCM & $1: 1.2$ & no rct. \\
\hline 15 & $\left(\mathrm{Ph}_{3} \mathrm{C}\right)\left(\mathrm{BF}_{4}\right)(10 \mathrm{~mol} \%)$ & DCM & $1: 1.2$ & $76 \%$ \\
\hline $16^{[\mathrm{d}]}$ & $\mathrm{HBF}_{4}(10 \mathrm{~mol} \%)$ & DCM & $1: 1.2$ & $28 \%$ \\
\hline $17^{[\mathrm{e}]}$ & $\mathrm{HBF}_{4}(10 \mathrm{~mol} \%)$ & DCM & $1: 1.2$ & $36 \%$ \\
\hline $18^{[\mathrm{d}]}$ & $\mathrm{HBF}_{4}(5 \mathrm{~mol} \%)$ & DCM & $1: 1.2$ & $14 \%$ \\
\hline $19^{[\mathrm{e}]}$ & $\mathrm{HBF}_{4}(5 \mathrm{~mol} \%)$ & DCM & $1: 1.2$ & $23 \%$ \\
\hline $20^{[\mathrm{f}]}$ & $\mathrm{HBF}_{4}(0.5 \mathrm{~mol} \%)$ & DCM & $1: 1.2$ & $\operatorname{trace} e^{[\mathrm{c}]}$ \\
\hline $21^{[\mathrm{g}]}$ & Trop(BF 4$)(10 \mathrm{~mol} \%)$ & DCM & $1: 1.2$ & $\operatorname{trace} e^{[\mathrm{c}]}$ \\
\hline $22^{[\mathrm{h}]}$ & Trop(BF 4$)(10 \mathrm{~mol} \%)$ & DCM & $1: 1.2$ & $7 \%[\mathrm{c}]$ \\
\hline $23^{[\mathrm{i}]}$ & Trop(BF 4$)\left(10 \mathrm{~mol}^{\circ} \%\right)$ & DCM & $1: 1.2$ & $9 \%[\mathrm{c}]$ \\
\hline $24^{[\mathrm{j}]}$ & Trop $\left(\mathrm{BF}_{4}\right)\left(10 \mathrm{~mol}^{\circ}\right)$ & DCM & $1: 1.2$ & $18 \%$ \\
\hline $25^{[\mathrm{k}]}$ & Trop $\left(\mathrm{BF}_{4}\right)\left(10 \mathrm{~mol}^{2} \mathrm{o}\right)$ & DCM & $1: 1.2$ & $39 \%$ \\
\hline $26^{[1]}$ & Trop $\left(\mathrm{BF}_{4}\right)(10 \mathrm{~mol} \%)$ & DCM & $1: 1.2$ & $90 \%$ \\
\hline
\end{tabular}

reaction conditions: ${ }^{[\mathrm{a}]}$ To a mixture of catalyst and benzoic acid $\mathbf{6 a}$ a solution of methyl phenyldiazoacetat 1a in $1.0 \mathrm{~mL}$ solvent was added and the resulting mixture was stirred until the orange color of the diazoalkane disappeared (approximate 10 min for DCM as solvent). ${ }^{[b]}$ isolated yield of product. ${ }^{[c]}$ NMR-yield using 0.2 mmol mesitylene as internal standard. ${ }^{[d]}$ yield after 10 min reaction time. ${ }^{[\mathrm{e}]}$ yield after full conversion of diazoalkane (approximate $45 \mathrm{~min}-1 \mathrm{~h}$ ). ${ }^{[\mathrm{f}]}$ yield after $24 \mathrm{~h}$ reaction time, no full conversion of diazoalkane observed. ${ }^{[\mathrm{g}]}$ reaction with 10 eq. water. ${ }^{[\mathrm{h}]}$ reaction with 2 eq. additional water. ${ }^{[\mathrm{i}]}$ reaction with 1 eq. water. ${ }^{[j]}$ reaction with 0.5 eq. water. ${ }^{[j]}$ reaction with 0.1 eq. water. ${ }^{[1]}$ reaction in the presence of $4 \AA$ molecular sieves. 


\section{General Procedures}

\section{General Procedure for O-H Functionalization of Benzoic Acids GP-1}

An oven-dried test tube was loaded with tropylium tetrafluoroborate $(10 \mathrm{~mol} \%)$ and the corresponding carboxylic acid derivative 6 or 11 (1.2 eq.; $0.22 \mathrm{mmol})$. The test tube was flushed and refilled with Ar for three times. Diazoalkane 1 (1.0 eq.; $0.2 \mathrm{mmol}$ ) dissolved in $1 \mathrm{~mL}$ dry, degassed DCM was added at once. The reaction mixture was stirred at room temperature until the orange color of the diazoalkane disappeared. The product was purified by silica column chromatography using $n$-hexane : ethyl acetate as eluent.

\section{General Procedure for Synthesis of Aryldiazoacetates GP-2}

A mixture of ester (1.0 eq., $5 \mathrm{mmol})$ and $p$-toluenesulfonyl azide $(1.2$ eq., $6 \mathrm{mmol})$ was dissolved in $30 \mathrm{~mL} \mathrm{MeCN}$ and cooled with an ice bath. DBU (1.4 eq., $7 \mathrm{mmol}$ ) were added. The reaction mixture was stirred overnight, while slowly warming up to room temperature. The reaction mixture was quenched by adding saturated aq. $\mathrm{NH}_{4} \mathrm{Cl}$. The solution was extracted with $\mathrm{DCM}$ and the organic layer was dried over $\mathrm{MgSO}_{4}$ and concentrated under reduced pressure. The product was purified by silica column chromatography n-pentane /Et2O $(80: 1 \rightarrow 40: 1)$.

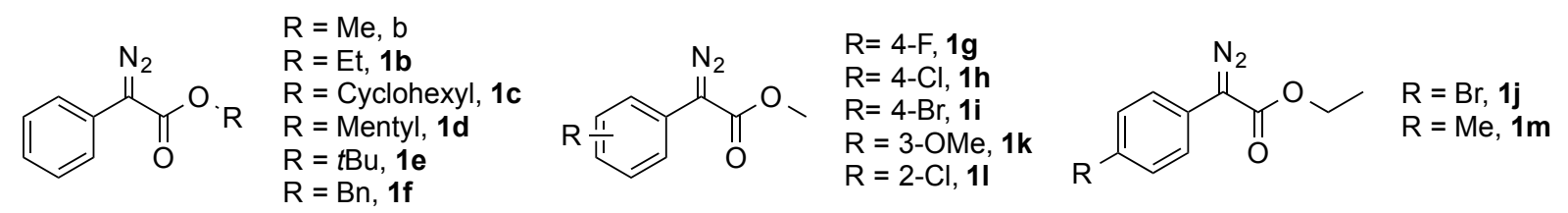

All aryldiazoacetates used in this manuscript are known in the literature.

For compound $\mathbf{1 a}, \mathbf{1 b}, \mathbf{1 f}, \mathbf{1 g}, \mathbf{1 h}, \mathbf{1 i}$ and $\mathbf{1 l}$ please see reference 2 .

For compound 1c, $1 \mathbf{f}$ and $1 \mathbf{k}$ please see reference 3.

For compound $\mathbf{1 d}$,and $\mathbf{1 m}$ please see reference 4 .

For compound $\mathbf{1} \mathbf{j}$, please see reference 5 .

\section{1 mmol Scale Experiment}

An oven-dried test tube was loaded with tropylium tetrafluoroborate $(10 \mathrm{~mol} \%, 0.1 \mathrm{mmol}, 17.8$ $\mathrm{mg}$ ) and the corresponding carboxylic acid derivative 6a (1.2 eq.; $1.1 \mathrm{mmol}, 147 \mathrm{mg})$. The test tube was flushed and refilled with Ar for three times. Diazoalkane 1a (1.0 eq.; 1 mmol, 176 $\mathrm{mg}$ ) dissolved in $10 \mathrm{~mL}$ dry, degassed DCM was added at once. The reaction mixture was stirred at room temperature until the orange color of the diazoalkane disappeared. The product 7a was purified by silica column chromatography using $n$-hexane : ethyl acetate as eluent and isolated as a colorless oil in $88 \%(239 \mathrm{mg})$. 


\section{Preparation of benzoic acid- $d(d-6 a)$}

An oven-dried test tube was loaded with benzoic acid (147 mg, $1.2 \mathrm{mmol})$ and dissolved in $0.5 \mathrm{~mL}$ methanol- $d_{1}$. The reaction mixture was stirred for 30 mins. Then the solvent was evaporated completely. In a next step another $0.5 \mathrm{~mL}$ methanol- $d_{1}$ was added and the reaction mixture was stirred for 30 mins, after evaporation, the cycle was repeated for another 5 times. A quantitative incorporation of deuterium was observed by ${ }^{1} \mathrm{H}$ NMR spectroscopy, furthermore, no residues peak of water was observed.

${ }^{1}$ H NMR (400 MHz, Chloroform- $d$ ): $\delta=8.28-8.00(\mathrm{~m}, 2 \mathrm{H}), 7.67-7.53(\mathrm{~m}, 1 \mathrm{H}), 7.54-7.38$ $(\mathrm{m}, 2 \mathrm{H}) \mathrm{ppm}$.

${ }^{1}$ H NMR (400 MHz, Chloroform- $d$ )

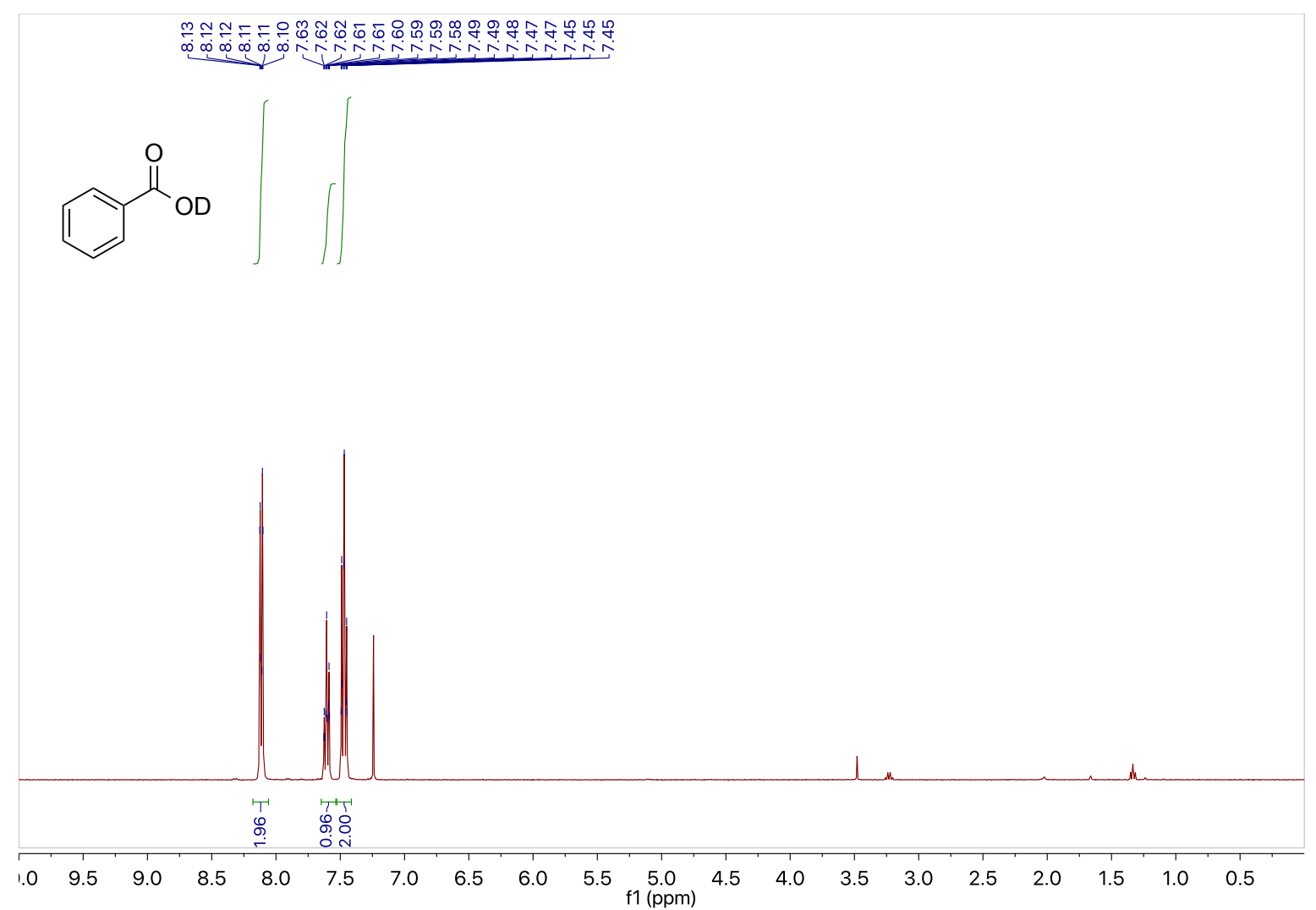

\section{Deuterium Labelling Experiments}

\section{Reaction in deuterated DCM}

An oven-dried test tube was loaded with tropylium tetrafluoroborate $(10 \mathrm{~mol} \%)$ and benzoic acid 6a (1.2 eq.; $0.22 \mathrm{mmol})$. The test tube was flushed and refilled with Ar for three times. Then methyl 2-diazo-2-phenylacetate 1a (1.0 eq.; $0.2 \mathrm{mmol})$ dissolved in $1 \mathrm{~mL}$ dry, degassed $d^{2}$-DCM was added at once. The reaction mixture was stirred at room temperature until the orange color of the diazoalkane disappeared. The product was purified by preparative silica thin layer chromatography using $n$-hexane : ethyl acetate (9:1) as eluent.

No incorporation of deuterium was observed by NMR of the crude reaction mixture. 


\section{Reaction with benzoic acid- $d(d-6 a)$}

An oven-dried test tube was loaded with tropylium tetrafluoroborate $(10 \mathrm{~mol} \%)$ and benzoic acid- $d$ (d-6a) (1.2 eq.; $0.22 \mathrm{mmol})$. The test tube was flushed and refilled with Ar for three times. Than methyl 2-diazo-2-phenylacetate 1a (1.0 eq.; $0.2 \mathrm{mmol})$ dissolved in $1 \mathrm{~mL}$ dry, degassed DCM was added at once. The reaction mixture was stirred at room temperature until the orange color of the diazoalkane disappeared. The product $\boldsymbol{d - 7} \mathbf{a}$ was purified by preparative silica thin layer chromatography using $n$-hexane : ethyl acetate $(9: 1)$ as eluent.

An incorporation of $66 \%$ deuterium was observed. $\mathrm{H}: \mathrm{D}=34: 66$

\section{2-Methoxy-2-oxo-1-phenylethyl-1- $d$ benzoate $(d-7$ a)}

${ }^{1}$ H NMR (400 MHz, Chloroform- $d$ )

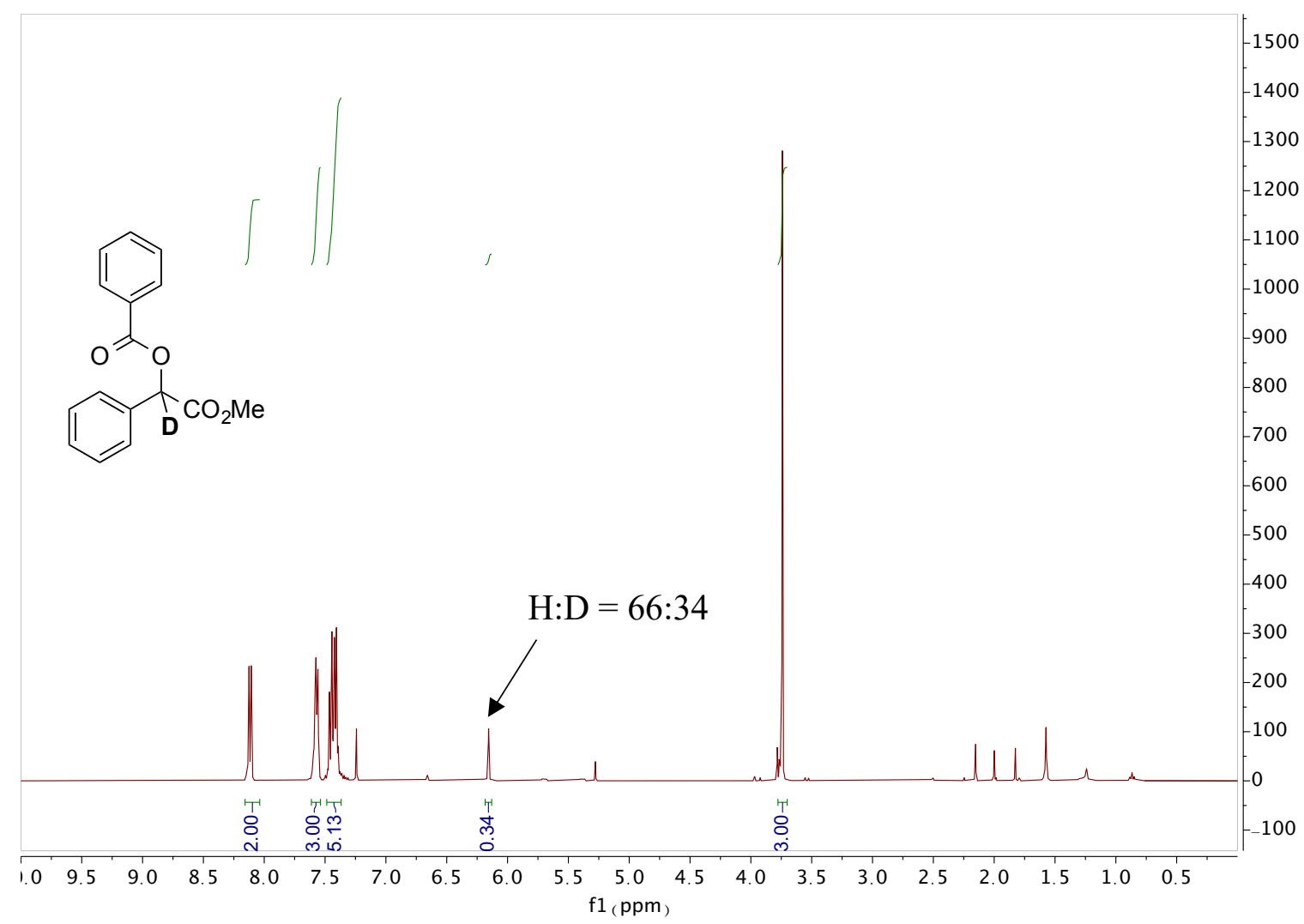




\section{Control Experiments on Activation Mode for Product 8 and 9}

An oven-dried test tube was loaded with catalyst (10 mol\%) and carboxylic acid 6a (1.2 eq.; $0.22 \mathrm{mmol})$. The test tube was flushed and refilled with Ar for three times. Diazoalkane 1n or 10 (1.0 eq.; $0.2 \mathrm{mmol}$ ) was dissolved in $1 \mathrm{~mL}$ dry, degassed DCM and was added at once. The reaction mixture was stirred at room temperature until the orange color of the diazoalkane disappeared. The product was purified by silica column chromatography using $n$-hexane : ethyl acetate as eluent.

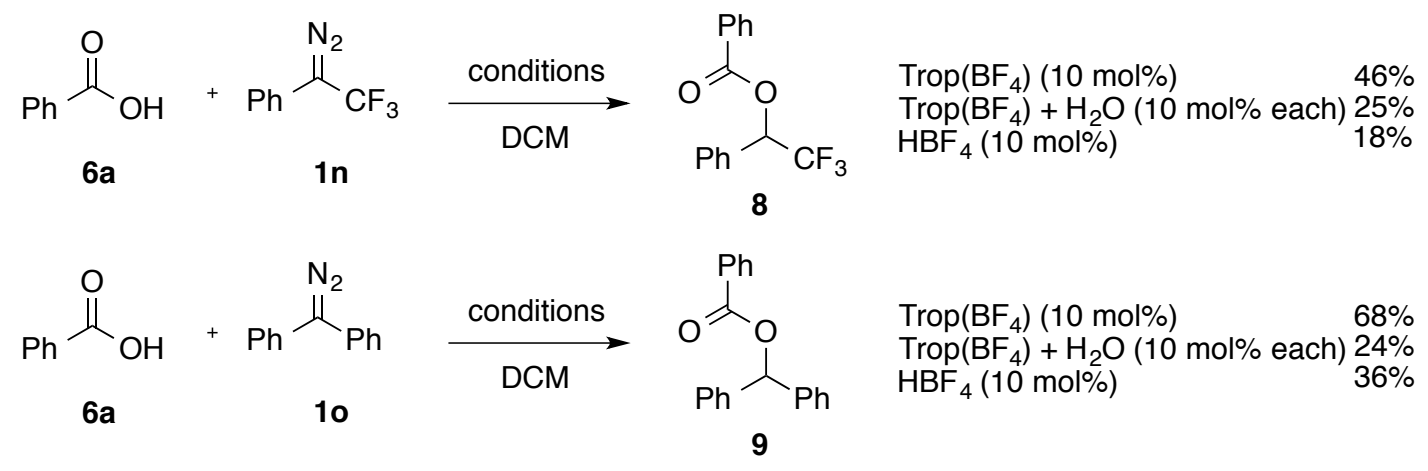




\section{Chemical Shift Perturbation Experiments}

A $0.1 \mathrm{M}$ solution in deuterated MeCN of a $1: 1$ mixture of tropylium tetrafluoroborate (19) with benzoic acid (6a) and methyl 2-diazo-2-phenylacetate (1a), respectively was investigate by ${ }^{1} \mathrm{H}$ and ${ }^{13} \mathrm{C}$ NMR spectroscopy.

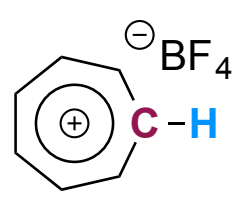

19

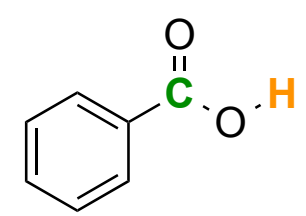

$6 a$

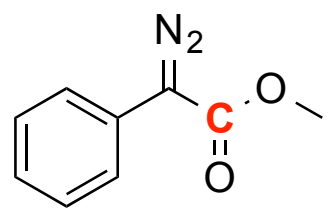

$1 \mathrm{a}$

\begin{tabular}{|c|c|c|c|c|c|c|c|c|c|c|c|}
\hline \# & substrates & $\delta(\mathrm{H}) \mathrm{ppm}$ & $\Delta \delta(\mathbf{H}) \mathrm{ppm}$ & $\delta(\mathbf{C}) \mathrm{ppm}$ & $\Delta \delta(\mathbf{C}) \mathrm{ppm}$ & $\delta(\mathbb{H}) \mathrm{ppm}$ & $\Delta \delta(\mathrm{H}) \mathrm{ppm}$ & $\delta(\mathbf{C}) \mathrm{ppm}$ & $\Delta \delta(\mathrm{C}) \mathrm{ppm}$ & $\delta(\mathrm{C}) \mathrm{ppm}$ & $\Delta \delta(\mathrm{C}) \mathrm{ppm}$ \\
\hline 1 & 19 & 9.25 & - & 156.53 & - & - & - & - & - & - & - \\
\hline 2 & $6 \mathbf{a}$ & - & - & - & - & 3.00 & - & 168.03 & - & - & - \\
\hline 3 & $1 \mathbf{a}$ & - & - & - & - & - & - & - & - & 166.45 & - \\
\hline 4 & $19+6 a$ & 9.24 & -0.01 & 156.34 & -0.19 & 2.92 & -0.08 & 168.04 & 0.01 & - & - \\
\hline 5 & $6 a+1 a$ & - & - & - & - & 3.12 & 0.12 & 168.06 & 0.03 & 166.45 & 0.00 \\
\hline
\end{tabular}

* Note: The interaction of 19 and 1a cannot be analyzed by chemical shift perturbation studies as 1a decomposes rapidly in the presence of 19. 


\section{Tropylium-BF 4 (19)}

${ }^{1}$ H NMR (400 MHz, Acetonitrile- $d_{3}$ )

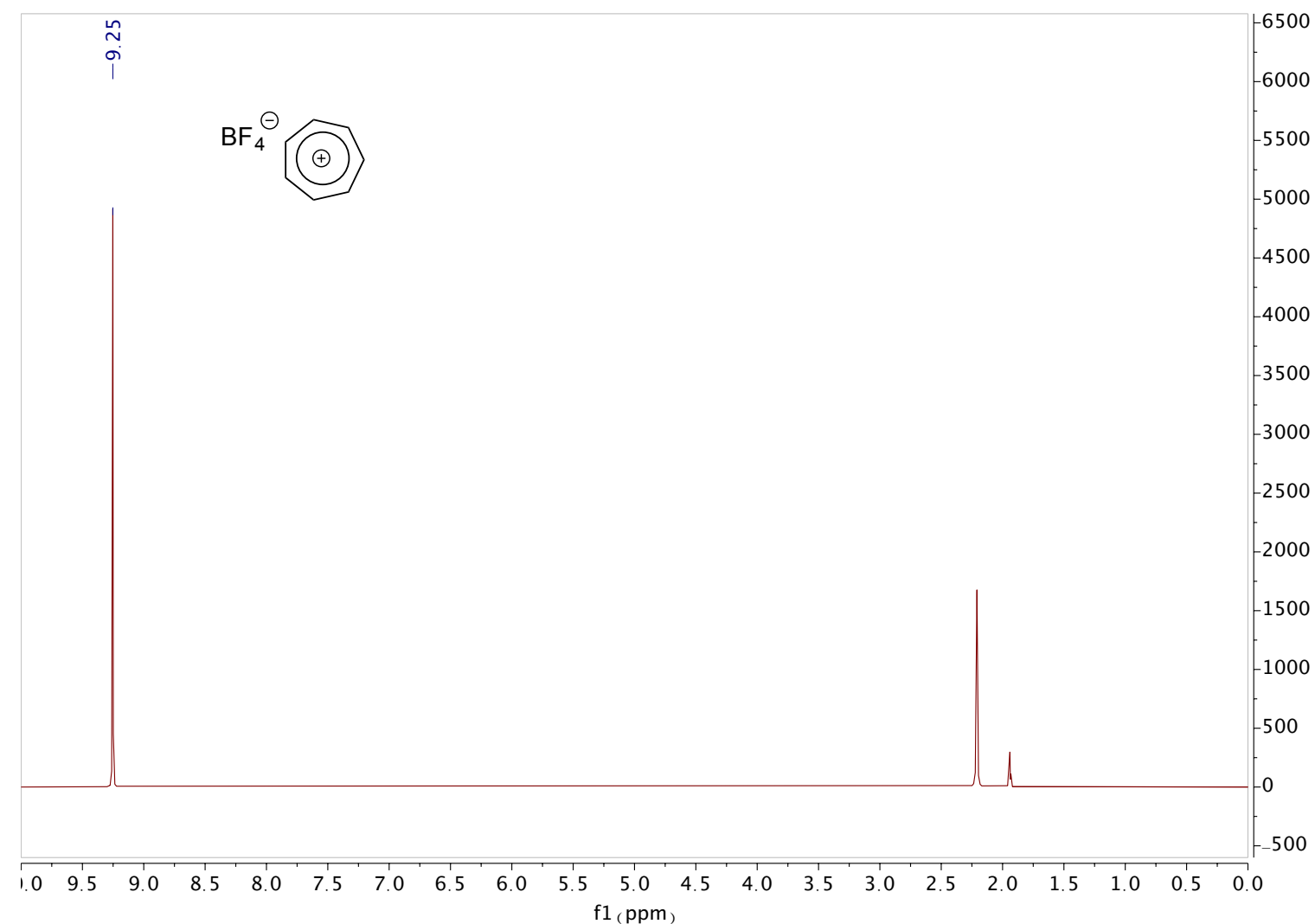

${ }^{13}$ C NMR (101 MHz, Acetonitrile- $\left.d_{3}\right)$

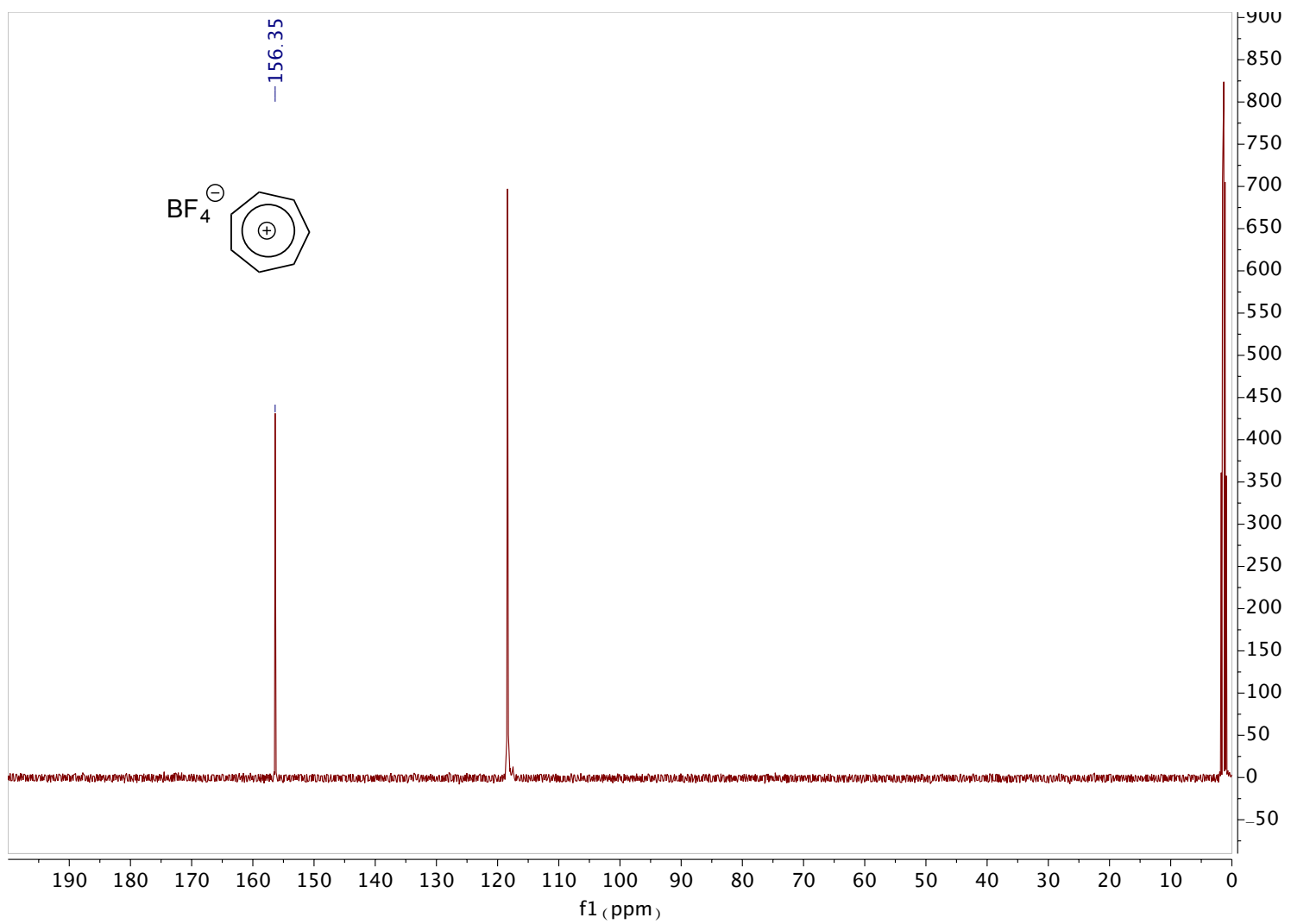




\section{Benzoic Acid (6a)}

${ }^{1}$ H NMR (400 MHz, Acetonitrile- $d_{3}$ )

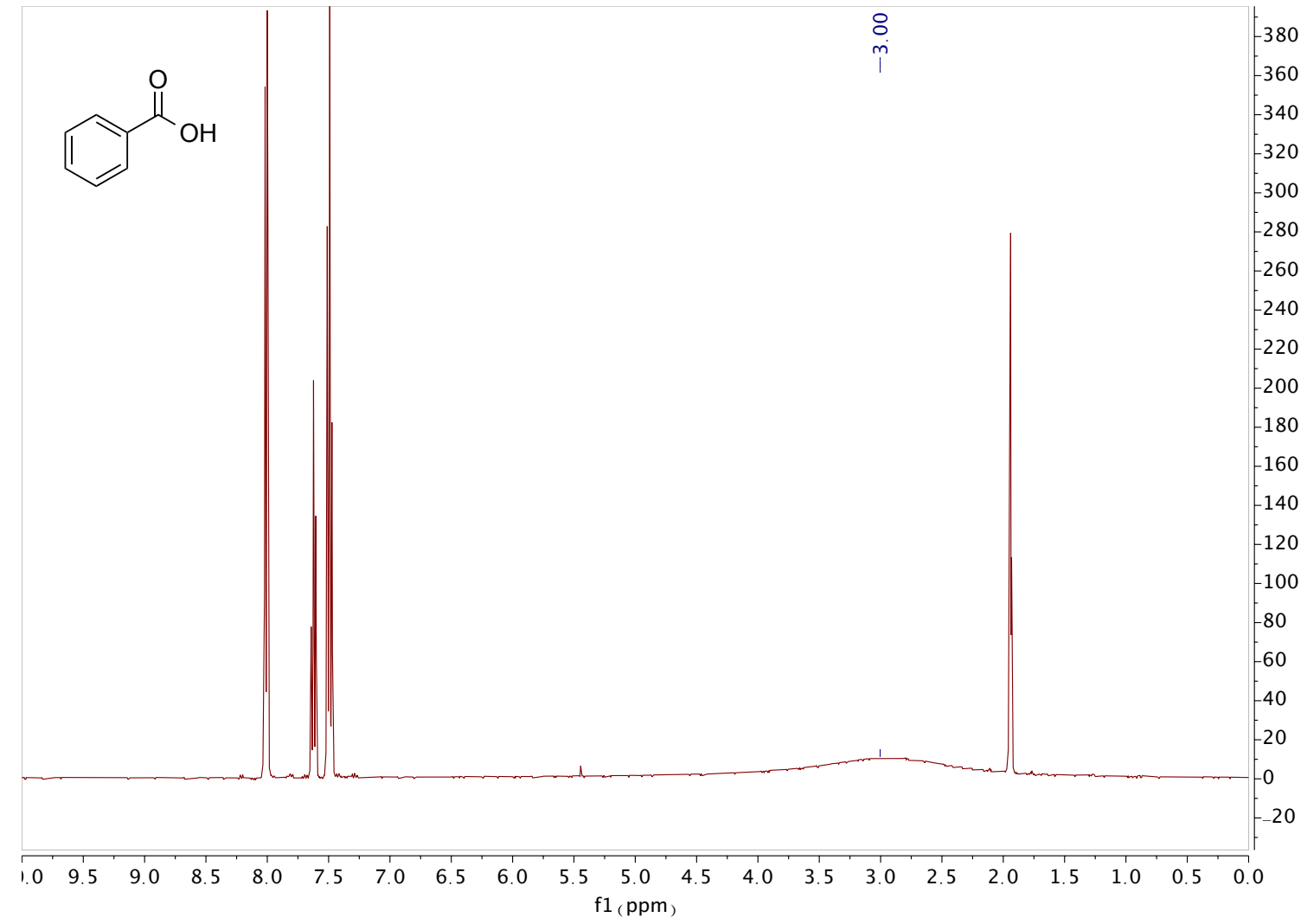

${ }^{13}$ C NMR (101 MHz, Acetonitrile- $\left.d_{3}\right)$

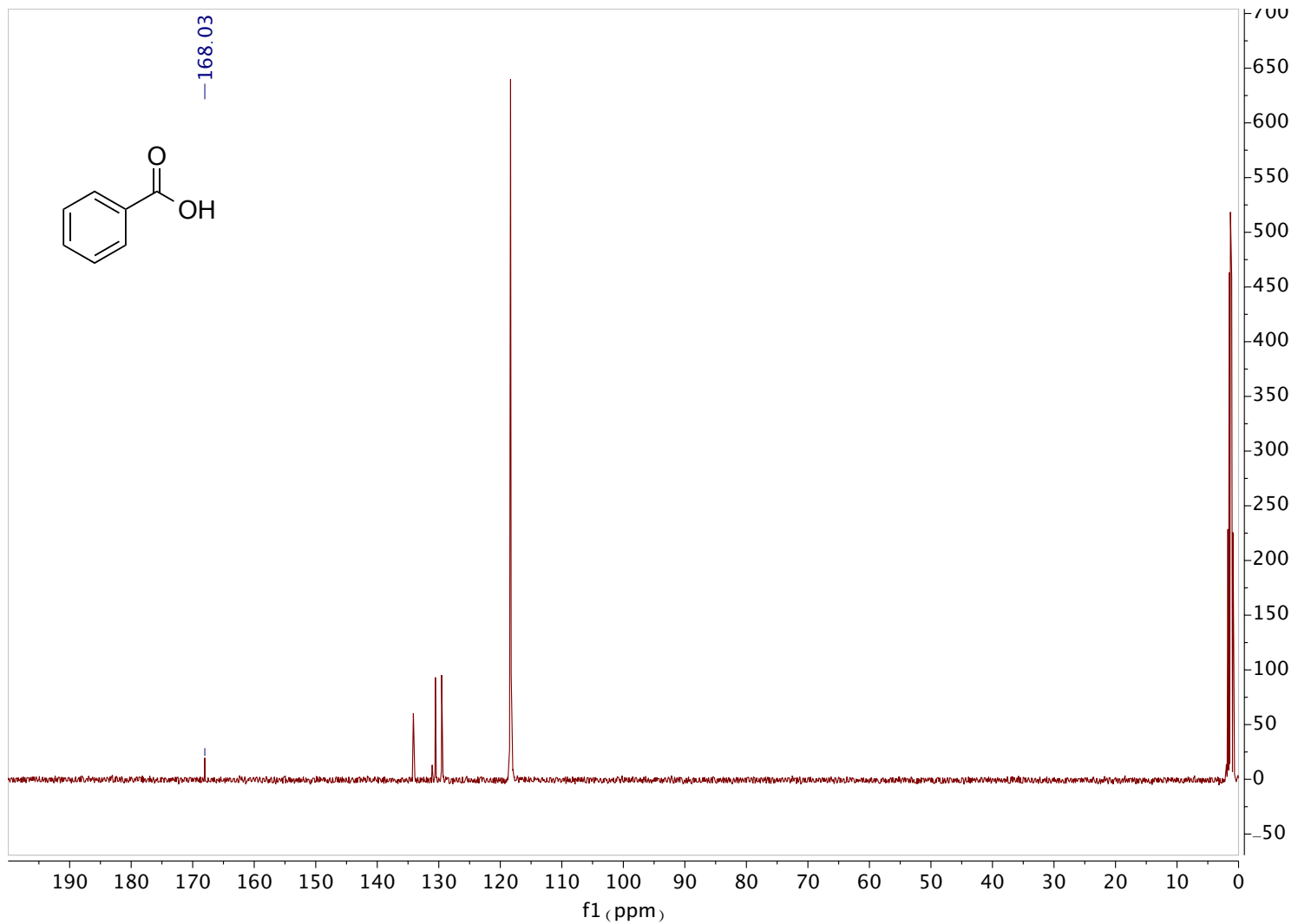


Methyl 2-diazo-2-phenylacetate (1a)

${ }^{1}$ H NMR (400 MHz, Acetonitrile- $d_{3}$ )

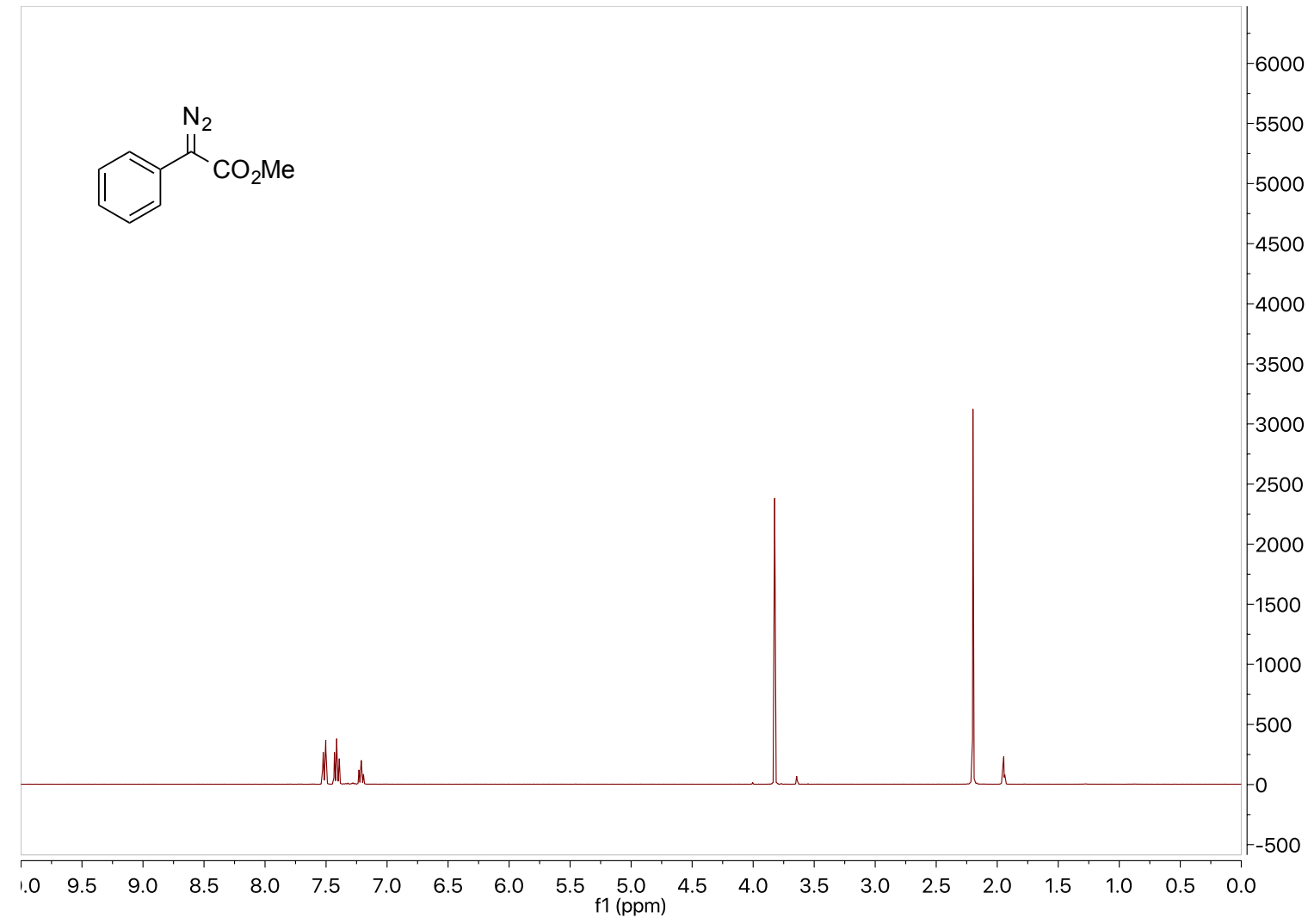

${ }^{13}$ C NMR (101 MHz, Acetonitrile- $\left.d_{3}\right)$

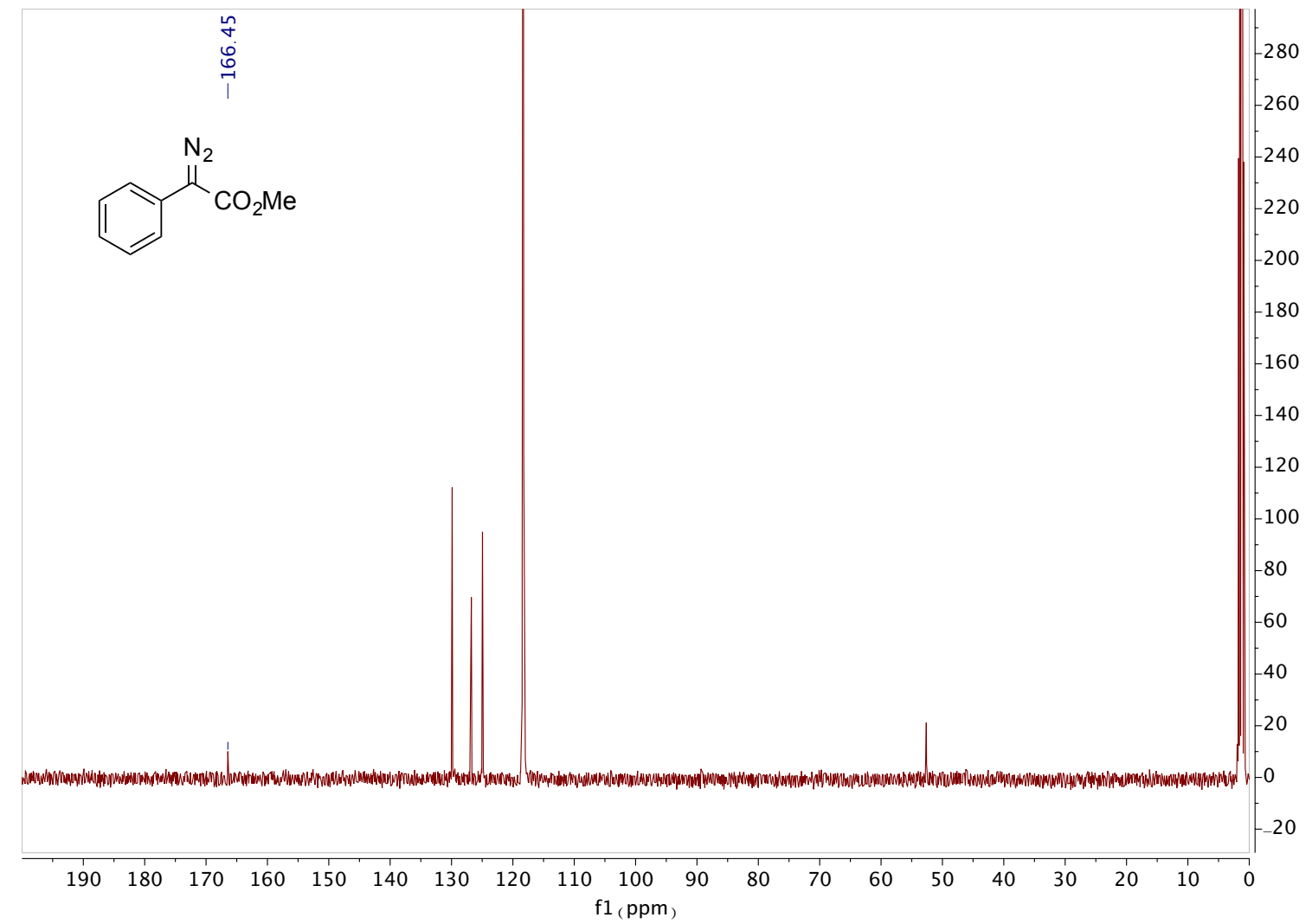


Tropylium-BF 4 (19) + Benzoic Acid (6a)

${ }^{1}$ H NMR (400 MHz, Acetonitrile- $d_{3}$ )

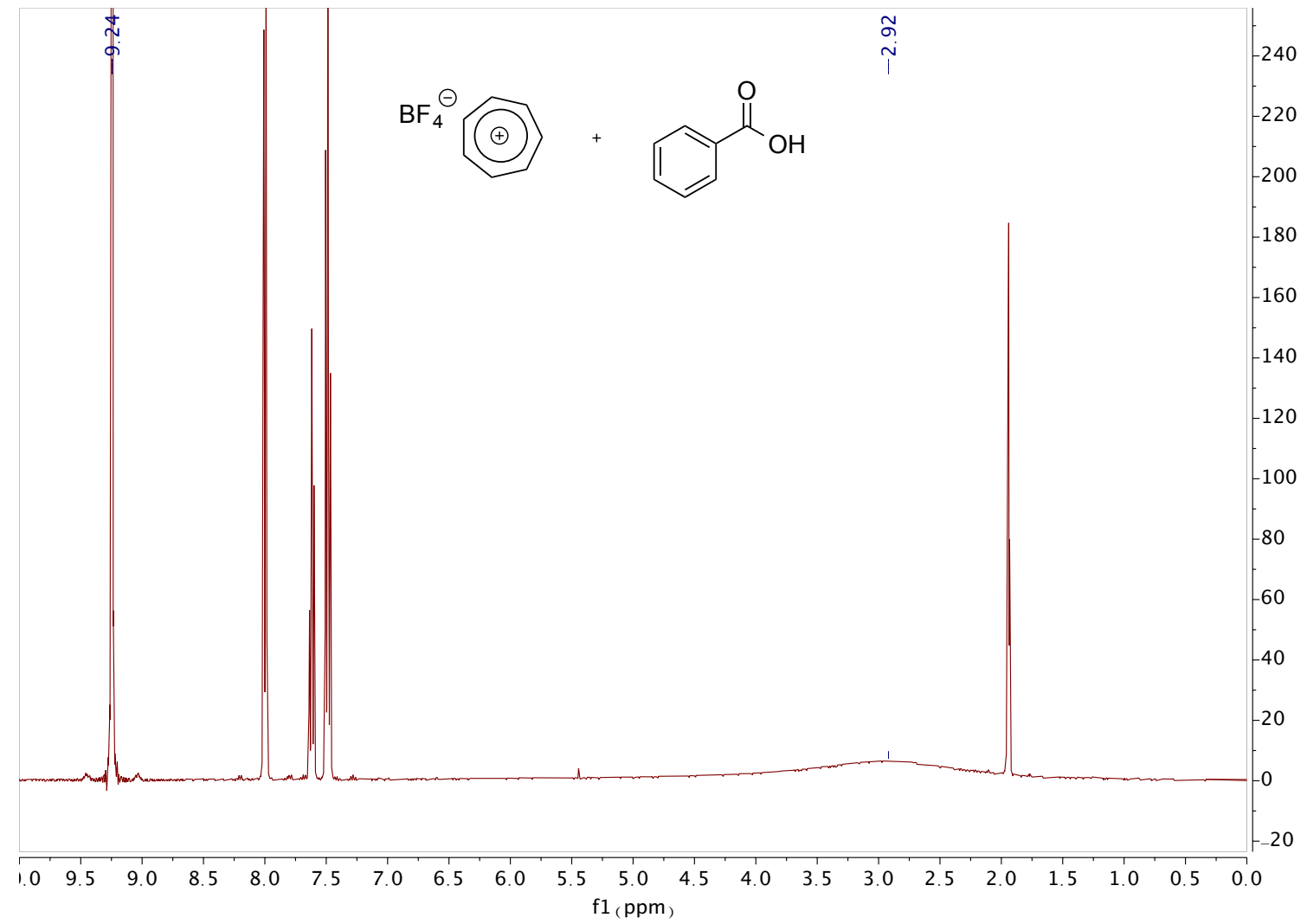

${ }^{13}$ C NMR (101 MHz, Acetonitrile- $\left.d_{3}\right)$

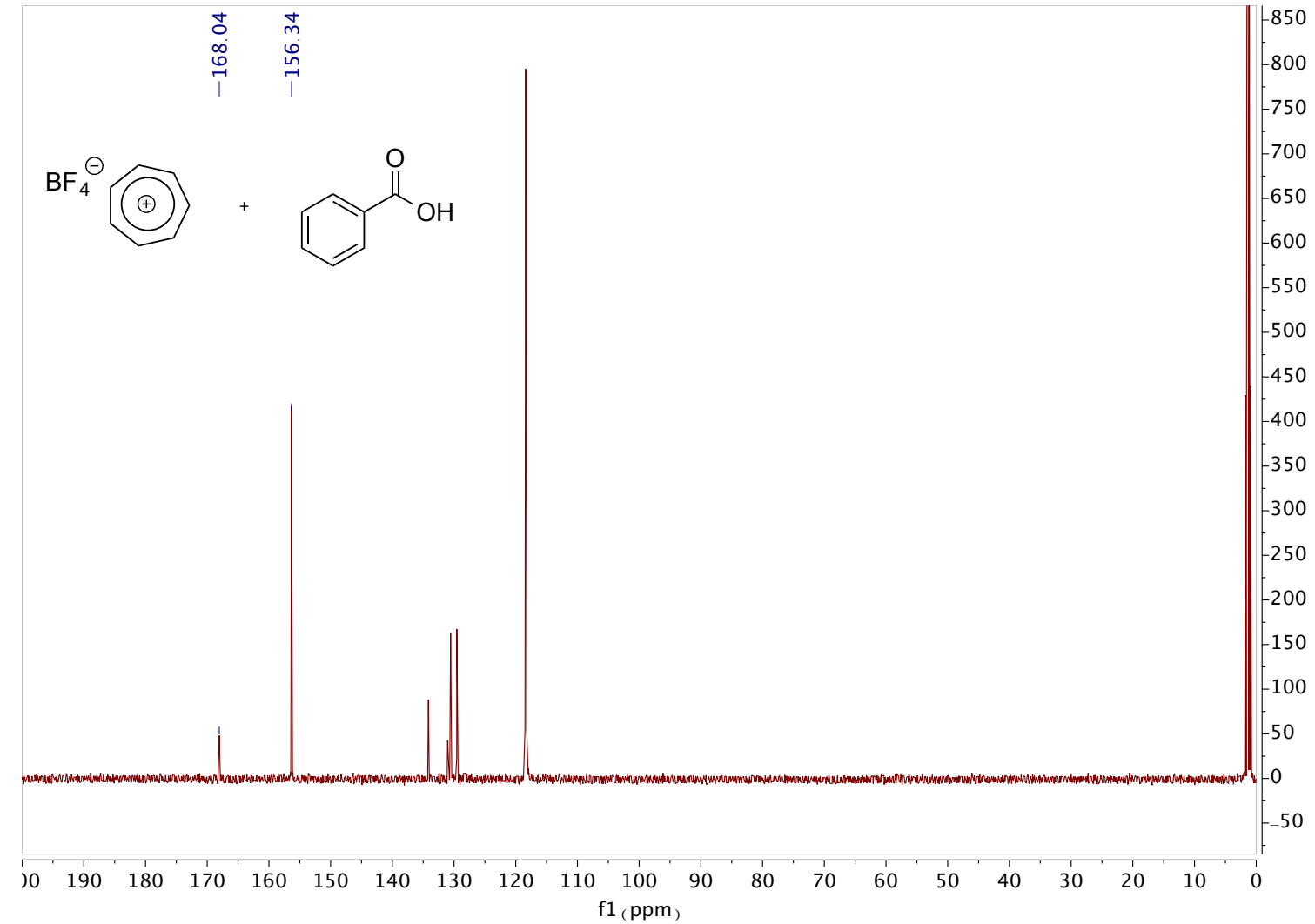


Benzoic Acid (6a) + Methyl 2-diazo-2-phenylacetate (1a)

${ }^{1}$ H NMR (400 MHz, Acetonitrile- $d_{3}$ )

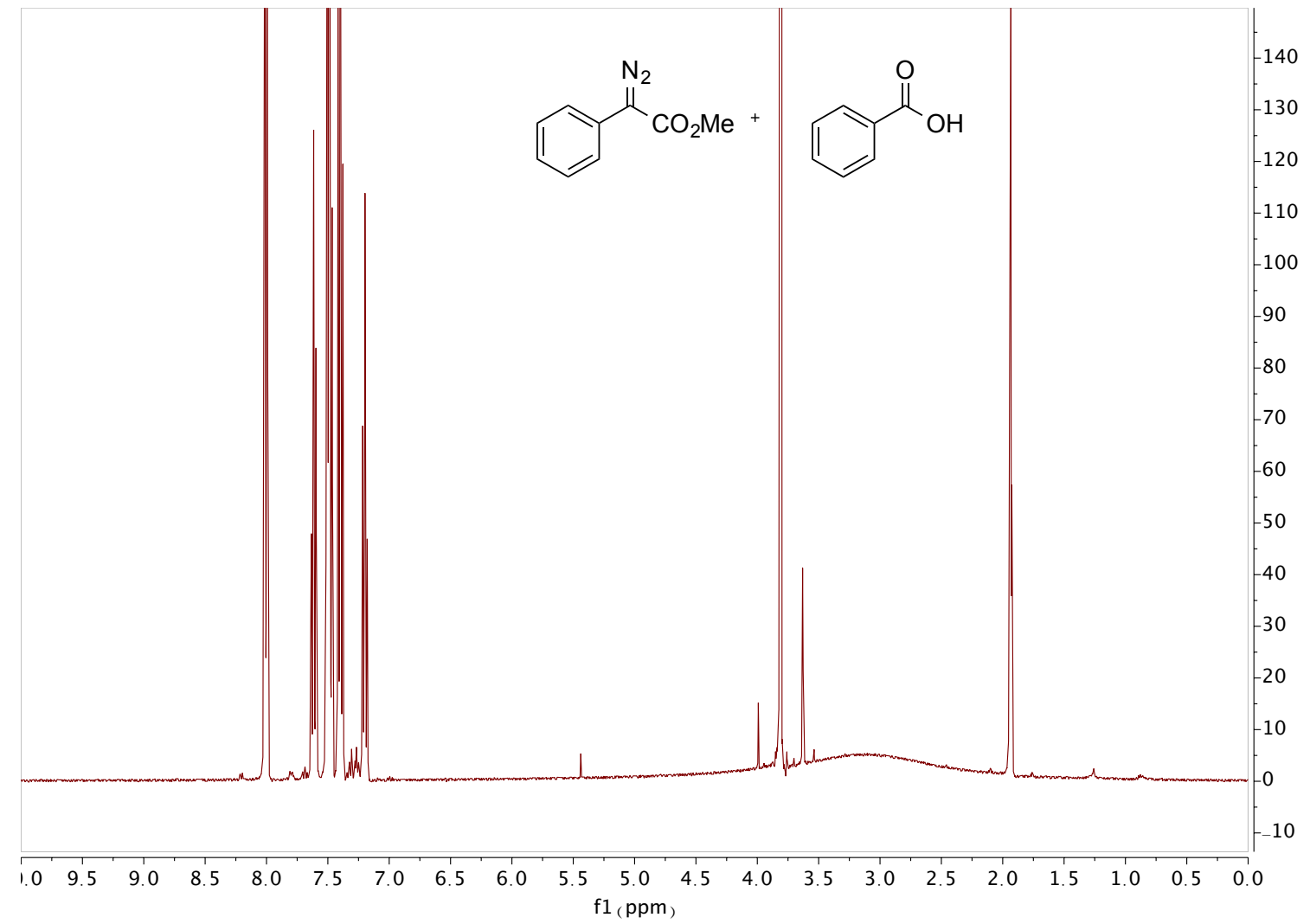

${ }^{13}$ C NMR (101 MHz, Acetonitrile- $\left.d_{3}\right)$

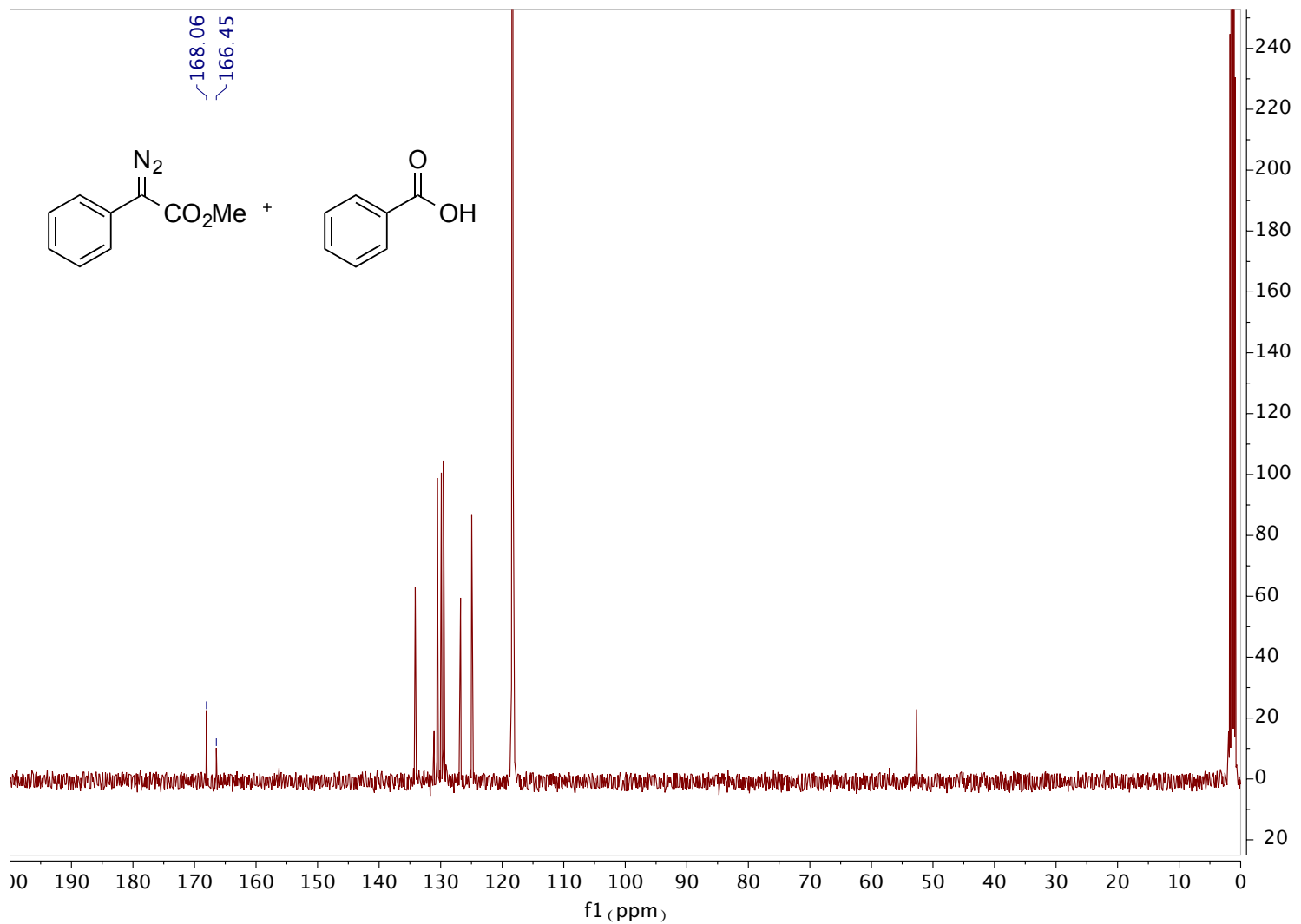




\section{Physical Data}

\section{2-Methoxy-2-oxo-1-phenylethyl benzoate (7a)}<smiles>COC(=O)C(OC(=O)c1ccccc1)c1ccccc1</smiles>

Compound 7a was prepared according to the general procedure GP-1 on a $0.2 \mathrm{mmol}$ scale and was obtained after silica column chromatography using $n$-hexane : EtOAc (40:1 $\rightarrow$ 9:1); 92\%, (49.7 mg); colorless oil.

${ }^{1}$ H NMR $(600 \mathrm{MHz}$, Chloroform- $d): \delta=\delta 8.17-8.09(\mathrm{~m}, 2 \mathrm{H}), 7.64-7.53(\mathrm{~m}, 3 \mathrm{H}), 7.51-$ $7.38(\mathrm{~m}, 5 \mathrm{H}), 6.17(\mathrm{~s}, 1 \mathrm{H}), 3.76(\mathrm{~s}, 3 \mathrm{H}) \mathrm{ppm}$.

${ }^{13}$ C NMR (151 MHz, Chloroform- $d$ ): $\delta=169.2,165.8,133.9,133.4,129.9,129.29,129.22$, $128.8,128.4,127.6,74.8,52.6 \mathrm{ppm}$.

HRMS (APCI): $m / z:[\mathrm{M}+\mathrm{H}]^{+}$Calcd. for $\mathrm{C}_{16} \mathrm{H}_{15} \mathrm{O}_{4}{ }^{+}: 271.0964$; Found: 271.0970 .

IR (KBr): 3446, 3065, 3034, 2954, 2847, 2657, 2328, 2116, 1917, 1819, 1722, 1599, 1494, $1449,1348,1316,1254,1216,1174,1104,1071,1029,970,913,851,778,704 \mathrm{~cm}^{-1}$.

\section{2-Ethoxy-2-oxo-1-phenylethyl benzoate (7b)}<smiles>CCOC(=O)C(OC(=O)c1ccccc1)c1ccccc1</smiles>

Compound 7b was prepared according to the general procedure GP-1 on a $0.2 \mathrm{mmol}$ scale and was obtained after silica column chromatography using $n$-hexane : EtOAc (40:1 $\rightarrow$ 9:1); 97\%, (55.1 mg); colorless oil.

${ }^{1}$ H NMR (400 MHz, Chloroform- $d$ ): $\delta=8.26-7.83(\mathrm{~m}, 2 \mathrm{H}), 7.69-7.50(\mathrm{~m}, 3 \mathrm{H}), 7.50-7.27$ $(\mathrm{m}, 5 \mathrm{H}), 4.48-3.98(\mathrm{~m}, 2 \mathrm{H}), 1.15(\mathrm{t}, J=7.1 \mathrm{~Hz}, 3 \mathrm{H}) \mathrm{ppm}$.

${ }^{13}$ C NMR (101 MHz, Chloroform- $d$ ): $\delta=168.8,165.9,134.1,133.4,129.9,129.3,129.2,128.8$, 128.4, 127.6, 75.0, 61.7, $14.0 \mathrm{ppm}$.

HRMS (ESI): $m / z$ : $[\mathrm{M}+\mathrm{Na}]^{+}$Calcd. for $\mathrm{C}_{17} \mathrm{H}_{16} \mathrm{NaO}_{4}{ }^{+}: 307.0940$; Found: 307.0937 .

IR (KBr): 3348, 2161, 1979, 1914, 1723, 1642, 1495, 1452, 1370, 1252, 1210, 1175, 1099 , $1035,943,907,702 \mathrm{~cm}^{-1}$. 


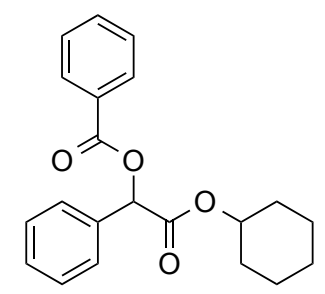

Compound 7c was prepared according to the general procedure GP-1 on a $0.2 \mathrm{mmol}$ scale and was obtained after silica column chromatography using $n$-hexane : EtOAc $(40: 1 \rightarrow 9: 1) ; 83 \%$, (56.2 mg); colorless oil.

${ }^{1}$ H NMR (400 MHz, Chloroform- $d$ ): $\delta=\delta 8.32-8.05(\mathrm{~m}, 2 \mathrm{H}), 7.68-7.54(\mathrm{~m}, 3 \mathrm{H}), 7.54-$ $7.35(\mathrm{~m}, 5 \mathrm{H}), 6.16(\mathrm{~s}, 1 \mathrm{H}), 4.88(\mathrm{dt}, J=8.6,4.4 \mathrm{~Hz}, 1 \mathrm{H}), 2.04-1.82(\mathrm{~m}, 1 \mathrm{H}), 1.76-1.65(\mathrm{~m}$, $2 \mathrm{H}), 1.59-1.44(\mathrm{~m}, 3 \mathrm{H}), 1.40-1.16(\mathrm{~m}, 4 \mathrm{H}) \mathrm{ppm}$.

${ }^{13}$ C NMR (101 MHz, Chloroform- $d$ ): $\delta=168.2,165.9,134.3,133.3,129.9,129.4,129.1,128.7$, $128.4,127.5,75.1,74.0,31.3,31.0,25.2,23.3,23.2 \mathrm{ppm}$.

HRMS (ESI): $m / z:[\mathrm{M}+\mathrm{K}]^{+}$Calcd. for $\mathrm{C}_{21} \mathrm{H}_{22} \mathrm{KO}_{4}{ }^{+}: 377.1149$; Found: 377.1144 .

IR (KBr): 3445, 3066, 3036, 2936, 2859, 2662, 2327, 2159, 2026, 1912, 1724, 1601, 1495, $1451,1381,1254,1212,1176,1104,1070,1030,968,909,831,806,705 \mathrm{~cm}^{-1}$.

\section{2-(((1R,2S,5R)-2-Isopropyl-5-methylcyclohexyl)oxy)-2-oxo-1-phenylethyl benzoate (7d)}<smiles>CC1CC[C@H](OC(=O)C(OC(=O)c2ccccc2)c2ccccc2)C(C(C)C)C1</smiles>

Compound 7d was prepared according to the general procedure GP-1 on a $0.2 \mathrm{mmol}$ scale and was obtained after silica column chromatography using $n$-hexane : EtOAc $(40: 1 \rightarrow 9: 1) ; 69 \%$, (54.2 mg); colorless oil; d.r. $\sim 1: 1.2$

${ }^{1}$ H NMR (400 MHz, Chloroform- $d$ ): $\delta=8.12-8.00(\mathrm{~m}, 2 \mathrm{H}), 7.61-7.46(\mathrm{~m}, 3 \mathrm{H}), 7.46-7.31$ $(\mathrm{m}, 5 \mathrm{H}), 6.06(\mathrm{~s}, 0.47 \mathrm{H})$ and $6.04(\mathrm{~s}, 0.53 \mathrm{H}), 4.67(\mathrm{td}, J=10.9,4.4 \mathrm{~Hz}, 0.50 \mathrm{H})$ and $4.59(\mathrm{td}, J$ $=10.9,4.4 \mathrm{~Hz}, 0.58 \mathrm{H}), 2.07-1.95(\mathrm{~m}, 0-58 \mathrm{H}), 1.94-1.84(\mathrm{~m}, 0.55 \mathrm{H}), 1.79-1.69(\mathrm{~m}, 0.53 \mathrm{H})$, $1.64-1.43(\mathrm{~m}, 2.62 \mathrm{H}), 1.44-1.28(\mathrm{~m}, 1.68 \mathrm{H}), 1.25-1.12(\mathrm{~m}, 1.32 \mathrm{H}), 1.07-0.86(\mathrm{~m}, 1.80 \mathrm{H})$, $0.82(\mathrm{~d}, J=6.8 \mathrm{~Hz}, 3.49 \mathrm{H}), 0.79-0.72(\mathrm{~m}, 2.62 \mathrm{H}), 0.72-0.65(\mathrm{~m}, 2.32 \mathrm{H}), 0.53(\mathrm{~d}, J=6.9$ $\mathrm{Hz}, 1.79 \mathrm{H})$ and $0.39(\mathrm{~d}, J=6.8 \mathrm{~Hz}, 1.75 \mathrm{H}) \mathrm{ppm}$

${ }^{13}$ C NMR (101 MHz, Chloroform- $\left.d\right)$ : $\delta=168.4,168.3,165.9,165.7,134.3,133.37,133.34$, $129.9,129.5,129.4,129.1,129.0,128.7,128.6,128.4,127.7,127.5,76.0,75.9,75.2,75.0,47.0$, $46.9,40.6,40.1,34.1,31.4,31.3,26.2,25.5,23.4,23.0,21.96,21.92,20.6,20.5,16.3,15.6$ ppm.

HRMS (ESI): $m / z$ : $[\mathrm{M}+\mathrm{K}]^{+}$Calcd. for $\mathrm{C}_{25} \mathrm{H}_{30} \mathrm{KO}_{4}{ }^{+}: 433.1775$; Found: 433.1767.

IR (KBr): 3448, 3066, 2954, 2869, 2661, 2330, 2186, 2109, 1988, 1913, 1726, 1601, 1494, $1453,1317,1255,1213,1176,1104,1030,980,957,911,842,791,735,705 \mathrm{~cm}^{-1}$. 
<smiles>CC(C)(C)OC(=O)C(OC(=O)c1ccccc1)c1ccccc1</smiles>

Compound 7e was prepared according to the general procedure GP-1 on a $0.2 \mathrm{mmol}$ scale and was obtained after silica column chromatography using $n$-hexane : EtOAc $(40: 1 \rightarrow 9: 1) ; 57 \%$, (35.7 mg); colorless oil.

${ }^{1}$ H NMR (400 MHz, Chloroform- $d$ ): $\delta=8.17-8.00(\mathrm{~m}, 2 \mathrm{H}), 7.57$ - $7.46(\mathrm{~m}, 3 \mathrm{H}), 7.45-7.32$ $(\mathrm{m}, 5 \mathrm{H}), 5.98(\mathrm{~s}, 1 \mathrm{H}), 1.34(\mathrm{~s}, 9 \mathrm{H}) \mathrm{ppm}$.

${ }^{13}$ C NMR (101 MHz, Chloroform- $d$ ): $\delta=167.8,165.8,134.6,133.3,129.9,129.5,128.9,128.7$, 128.4, 127.5, 82.5, 75.3, $27.8 \mathrm{ppm}$.

HRMS (ESI): $m / z:[\mathrm{M}+\mathrm{K}]^{+}$Calcd. for $\mathrm{C}_{19} \mathrm{H}_{20} \mathrm{KO}_{4}{ }^{+}: 351.0993$; Found: 351.0992.

IR (KBr): 3444, 3065, 3036, 2979, 2934, 2659, 2327, 2188, 2090, 1991, 1920, 1724, 1600, $1492,1452,1365,1254,1152,1105,1070,1027,956,910,843,794,740,706 \mathrm{~cm}^{-1}$.

\section{2-(Benzyloxy)-2-oxo-1-phenylethyl benzoate (7f)}<smiles>O=C(OC(C(=O)OCc1ccccc1)c1ccccc1)c1ccccc1</smiles>

Compound 7f was prepared according to the general procedure GP- 1 on a $0.2 \mathrm{mmol}$ scale and was obtained silica column chromatography using $n$-hexane : EtOAc $(40: 1 \rightarrow 9: 1) ; 70 \%,(48.2$ $\mathrm{mg})$; colorless oil.

${ }^{1}$ H NMR (400 MHz, Chloroform- $d$ ): $\delta=8.26-7.99(\mathrm{~m}, 2 \mathrm{H}), 7.61-7.42(\mathrm{~m}, 3 \mathrm{H}), 7.42-7.28$ (m, 5H), $7.26-7.09(\mathrm{~m}, 5 \mathrm{H}), 6.15(\mathrm{~s}, 1 \mathrm{H}), 5.31-4.92(\mathrm{~m}, 2 \mathrm{H}) \mathrm{ppm}$.

${ }^{13}$ C NMR (101 MHz, Chloroform- $d$ ): $\delta=168.6,165.8,135.2,133.9,133.4,130.0,129.2,128.8$, $128.49,128.47,128.2,127.8,127.7,74.9,67.2 \mathrm{ppm}$.

HRMS (ESI): $m / z$ : $[\mathrm{M}+\mathrm{K}]^{+}$Calcd. for $\mathrm{C}_{22} \mathrm{H}_{18} \mathrm{KO}_{4}{ }^{+}$: 385.0836 ; Found: 385.0834 .

IR (KBr): 3444, 3065, 3035, 2955, 2332, 2182, 1889, 1752, 1723, 1600, 1495, 1452, 1379, $1318,1254,1206,1171,1103,1071,1028,911,829,698 \mathrm{~cm}^{-1}$. 


\section{1-(4-Fluorophenyl)-2-methoxy-2-oxoethyl benzoate (7g)}<smiles>COC(=O)C(OC(=O)c1ccccc1)c1ccc(F)cc1</smiles>

Compound $7 \mathbf{g}$ was prepared according to the general procedure GP-1 on a $0.2 \mathrm{mmol}$ scale and was obtained after silica column chromatography using $n$-hexane : EtOAc (40:1 $\rightarrow$ 9:1); 68\%, (38.9 mg); colorless oil.

${ }^{1}$ H NMR $(600 \mathrm{MHz}$, Chloroform- $d$ ): $\delta=8.17-8.10(\mathrm{~m}, 2 \mathrm{H}), 7.68-7.53(\mathrm{~m}, 3 \mathrm{H}), 7.50-7.40$ (m, 2H), $7.17-7.08(\mathrm{~m}, 2 \mathrm{H}), 6.15(\mathrm{~s}, 1 \mathrm{H}), 3.76(\mathrm{~s}, 2 \mathrm{H}) \mathrm{ppm}$.

${ }^{13}$ C NMR (151 MHz, Chloroform- $d$ ): $\delta=169.1,165.7,163.2\left(\mathrm{~d}, \mathrm{C}-\mathrm{F},{ }^{1} J_{\mathrm{C}-\mathrm{F}}=248.7 \mathrm{~Hz}\right), 133.5$, $129.9,129.8\left(\mathrm{~d}, \mathrm{C}-\mathrm{F},{ }^{4} J_{\mathrm{C}-\mathrm{F}}=3.5 \mathrm{~Hz}\right), 129.5\left(\mathrm{~d}, \mathrm{C}-\mathrm{F},{ }^{3} J_{\mathrm{C}-\mathrm{F}}=8.4 \mathrm{~Hz}\right), 129.0,128.4,115.9$, (d, C$\left.\mathrm{F},{ }^{2} J_{\mathrm{C}-\mathrm{F}}=22.6 \mathrm{~Hz}\right), 74.1,52.7 \mathrm{ppm}$.

${ }^{19}$ F NMR (564 MHz, Chloroform- $d$ ): $\delta=-100.24--132.15$ (m) ppm.

HRMS (ESI): $m / z$ : $[\mathrm{M}+\mathrm{Na}]^{+}$Calcd. for $\mathrm{C}_{16} \mathrm{H}_{13} \mathrm{FNaO}_{4}{ }^{+}: 311.0690$; Found: 311.0685 .

IR (KBr): 3442, 3069, 2956, 2848, 2665, 2326, 2086, 1992, 1911, 1753, 1723, 1604, 1510, 1446, 1346, 1315, 1254, 1222, 1173, 1102, 1070, 1030, 970, 909, 837, 803, 755, $710 \mathrm{~cm}^{-1}$.

\section{1-(4-Chlorophenyl)-2-methoxy-2-oxoethyl benzoate (7h)}<smiles>COC(=O)C(OC(=O)c1ccccc1)c1ccc(Cl)cc1</smiles>

Compound $7 \mathbf{h}$ was prepared according to the general procedure GP-1 on a $0.2 \mathrm{mmol}$ scale and was obtained after silica column chromatography using $n$-hexane : EtOAc (40:1 $\rightarrow$ 9:1); 99\%, $(60.2 \mathrm{mg})$; colorless oil.

${ }^{1}$ H NMR (400 MHz, Chloroform- $d$ ): $\delta=8.17-8.09(\mathrm{~m}, 2 \mathrm{H}), 7.68-7.59(\mathrm{~m}, 1 \mathrm{H}), 7.58-7.52$ (m, 2H), $7.53-7.45(\mathrm{~m}, 2 \mathrm{H}), 7.46-7.40(\mathrm{~m}, 2 \mathrm{H}), 6.17(\mathrm{~s}, 1 \mathrm{H}), 3.78(\mathrm{~s}, 3 \mathrm{H}) \mathrm{ppm}$.

${ }^{13}$ C NMR (101 MHz, Chloroform- $d$ ): $\delta=168.9,165.7,135.3,133.6,132.5,129.9,129.1,129.0$, $128.9,128.5,74.1,52.8 \mathrm{ppm}$.

HRMS (APCI): $m / z$ : $[\mathrm{M}+\mathrm{H}]^{+}$Calcd. for $\mathrm{C}_{16} \mathrm{H}_{14} \mathrm{ClO}_{4}{ }^{+}$: 305.0575 ; Found: 305.0578.

IR (KBr): 3444, 3066, 2955, 2848, 2323, 2161, 2035, 1914, 1753, 1723, 1599, 1491, 1447, $1410,1345,1253,1217,1174,1095,1017,970,909,816,769,709 \mathrm{~cm}^{-1}$. 


\section{1-(4-Bromophenyl)-2-methoxy-2-oxoethyl benzoate (7i)}<smiles>COC(=O)C(OC(=O)c1ccccc1)c1ccc(Br)cc1</smiles>

Compound 7i was prepared according to the general procedure GP-1 on a $0.2 \mathrm{mmol}$ scale and was obtained after silica column chromatography using $n$-hexane : EtOAc $(40: 1 \rightarrow 9: 1) ; 80 \%$, (55.8 mg); colorless oil.

${ }^{1}$ H NMR (400 MHz, Chloroform- $d$ ): $\delta=8.10-7.95(\mathrm{~m}, 2 \mathrm{H}), 7.60-7.45(\mathrm{~m}, 3 \mathrm{H}), 7.41-7.36$ (m, 4H), 6.05 (s, 1H), 3.68 (s, 3H) ppm.

${ }^{13}$ C NMR (101 MHz, Chloroform- $d$ ): $\delta=168.8,165.7,133.6,133.0,132.0,129.9,129.2,129.0$, $128.5,123.5,74.1,52.8 \mathrm{ppm}$.

HRMS (ESI): $m / z:[\mathrm{M}+\mathrm{K}]^{+}$Calcd. for $\mathrm{C}_{16} \mathrm{H}_{13} \mathrm{BrKO}_{4}{ }^{+}: 386.9628$; Found: 386.9627.

IR (KBr): 3444, 3065, 2954, 2848, 2326, 2190, 2116, 1990, 1914, 1753, 1723, 1596, 1488, $1447,1405,1345,1254,1217,1174,1103,1070,1012,970,909,813,766,710 \mathrm{~cm}^{-1}$.

\section{1-(4-Bromophenyl)-2-ethoxy-2-oxoethyl benzoate (7j)}<smiles>CCOC(=O)C(OC(=O)c1ccccc1)c1ccc(Br)cc1</smiles>

Compound $7 \mathbf{j}$ was prepared according to the general procedure GP-1 on a $0.2 \mathrm{mmol}$ scale and was obtained after silica column chromatography using $n$-hexane : EtOAc $(40: 1 \rightarrow 9: 1) ; 75 \%$, $(54.3 \mathrm{mg})$; colorless oil.

${ }^{1}$ H NMR (400 MHz, Chloroform- $d$ ): $\delta=8.16-7.97(\mathrm{~m}, 2 \mathrm{H}), 7.62-7.43(\mathrm{~m}, 3 \mathrm{H}), 7.43-7.32$ (m, 4H), $6.03(\mathrm{~s}, 1 \mathrm{H}), 4.97-3.88(\mathrm{~m}, 2 \mathrm{H}), 1.16(\mathrm{t}, J=7.1 \mathrm{~Hz}, 3 \mathrm{H}) \mathrm{ppm}$.

${ }^{13}$ C NMR (101 MHz, Chloroform- $d$ ): $\delta=168.3,165.7,133.5,133.1,132.0,129.9,129.2,129.1$, $128.5,123.4,74.3,61.9,14.0 \mathrm{ppm}$.

HRMS (ESI): $m / z:[\mathrm{M}+\mathrm{K}]^{+}$Calcd. for $\mathrm{C}_{17} \mathrm{H}_{15} \mathrm{BrKO}_{4}{ }^{+}$: 400.9785 ; Found: 400.9783.

IR (KBr): 3445, 3066, 2982, 2327, 2172, 1912, 1724, 1596, 1488, 1451, 1401, 1369, 1340, $1253,1211,1175,1101,1070,1033,942,909,811,764,710 \mathrm{~cm}^{-1}$. 
<smiles>COC(=O)C(OC(=O)c1ccccc1)c1cccc(OC)c1</smiles>

Compound 7k was prepared according to the general procedure GP-1 on a $0.2 \mathrm{mmol}$ scale and was obtained after silica column chromatography using $n$-hexane : EtOAc $(40: 1 \rightarrow 9: 1)$; 94\%, $(56.7 \mathrm{mg})$; colorless oil.

${ }^{1}$ H NMR (400 MHz, Chloroform- $d$ ): $\delta=8.22-8.09(\mathrm{~m}, 2 \mathrm{H}), 7.67-7.56(\mathrm{~m}, 1 \mathrm{H}), 7.54-7.44$ $(\mathrm{m}, 2 \mathrm{H}), 7.37$ (t, $J=7.9 \mathrm{~Hz}, 1 \mathrm{H}), 7.19(\mathrm{dd}, J=7.6,1.3 \mathrm{~Hz}, 1 \mathrm{H}), 7.18-7.12(\mathrm{~m}, 1 \mathrm{H}), 7.02-$ $6.94(\mathrm{~m}, 1 \mathrm{H}), 6.17(\mathrm{~s}, 1 \mathrm{H}), 3.87(\mathrm{~s}, 3 \mathrm{H}), 3.79(\mathrm{~s}, 3 \mathrm{H}) \mathrm{ppm}$.

${ }^{13}$ C NMR (101 MHz, Chloroform- $d$ ): $\delta=169.2,165.8,159.9,135.3,133.5,130.0,129.9,129.2$, 128.4, 119.9, 114.8, 113.2, 74.7, 55.3, 52.7 ppm.

HRMS (ESI): $\mathrm{m} / z$ : $[\mathrm{M}+\mathrm{K}]^{+}$Calcd. for $\mathrm{C}_{17} \mathrm{H}_{16} \mathrm{KO}_{5}{ }^{+}: 339.0629$; Found: 339.0624.

IR (KBr): 3444, 3005, 2955, 2840, 2161, 2027, 1753, 1722, 1598, 1490, 1451, 1347, 1315 , $1260,1209,1173,1103,1034,975,861,780,743,708 \mathrm{~cm}^{-1}$.

\section{1-(2-Chlorophenyl)-2-methoxy-2-oxoethyl benzoate (7l)}<smiles>COC(=O)C(OC(=O)c1ccccc1)c1ccccc1Cl</smiles>

Compound $7 \mathbf{l}$ was prepared according to the general procedure GP-1 on a $0.2 \mathrm{mmol}$ scale and was obtained after silica column chromatography using $n$-hexane : EtOAc (40:1 $\rightarrow$ 9:1); 55\%, (33.4 mg); colorless oil.

${ }^{1}$ H NMR $(600 \mathrm{MHz}$, Chloroform- $d)$ : $\delta=8.23-7.92(\mathrm{~m}, 2 \mathrm{H}), 7.71-7.51(\mathrm{~m}, 2 \mathrm{H}), 7.51-7.38$ $(\mathrm{m}, 3 \mathrm{H}), 7.37-7.30(\mathrm{~m}, 2 \mathrm{H}), 6.71(\mathrm{~s}, 1 \mathrm{H}), 3.79(\mathrm{~s}, 3 \mathrm{H}) \mathrm{ppm}$.

${ }^{13}$ C NMR (151 MHz, Chloroform- $d$ ): $\delta=168.8,165.5,134.2,133.5,132.3,130.5,130.06$, 130.02, 129.6, 129.1, 128.4, 127.2, 71.4, $52.8 \mathrm{ppm}$.

HRMS (ESI): $m / z:[\mathrm{M}+\mathrm{K}]^{+}$Calcd. for $\mathrm{C}_{16} \mathrm{H}_{13} \mathrm{ClKO}_{4}{ }^{+}: 343.0133$; Found: 343.0132.

IR (KBr): 3447, 3067, 2954, 2160, 2025, 1755, 1724, 1599, 1476, 1444, 1346, 1318, 1249, $1218,1177,1097,1033,969,783,754,710 \mathrm{~cm}^{-1}$. 


\section{2-Ethoxy-2-oxo-1-(p-tolyl)ethyl benzoate $(7 \mathrm{~m})$}<smiles>CCOC(=O)C(OC(=O)c1ccccc1)c1ccc(C)cc1</smiles>

Compound $7 \mathbf{m}$ was prepared according to the general procedure GP- 1 on a $0.2 \mathrm{mmol}$ scale and was obtained after silica column chromatography using $n$-hexane : EtOAc $(40: 1 \rightarrow 9: 1) ; 78 \%$, (46.3 mg); colorless oil.

${ }^{1}$ H NMR $(600 \mathrm{MHz}$, Chloroform- $d)$ : $\delta=8.22-7.96(\mathrm{~m}, 2 \mathrm{H}), 7.66-7.53(\mathrm{~m}, 1 \mathrm{H}), 7.53-7.39$ $(\mathrm{m}, 4 \mathrm{H}), 7.24(\mathrm{~d}, J=7.8 \mathrm{~Hz}, 2 \mathrm{H}), 6.11(\mathrm{~s}, 1 \mathrm{H}), 4.30-4.13(\mathrm{~m}, 2 \mathrm{H}), 2.38(\mathrm{~s}, 3 \mathrm{H}), 1.36-1.05$ $(\mathrm{m}, 3 \mathrm{H}) \mathrm{ppm}$.

${ }^{13}$ C NMR (151 MHz, Chloroform- $d$ ): $\delta=168.9,165.9,139.1,133.3,131.1,129.9,129.5,129.3$, 128.4, 127.6, 74.9, 61.6, 21.2, $14.0 \mathrm{ppm}$.

HRMS (ESI): $m / z$ : $[\mathrm{M}+\mathrm{Na}]^{+}$Calcd. for $\mathrm{C}_{18} \mathrm{H}_{18} \mathrm{NaO}_{4}{ }^{+}: 321.1097$; Found: 321.1095 .

IR (KBr): 3444, 2982, 2328,2189, 2122, 1995, 1912, 1723, 1602, 1514, 1451, 1370, 1340, $1314,1254,1211,1176,1102,1070,1031,942,908,852,810,744,710 \mathrm{~cm}^{-1}$.

\section{2-Methoxy-2-oxo-1-phenylethyl 4-methylbenzoate (7n)}<smiles>COC(=O)C(OC(=O)c1ccc(C)cc1)c1ccccc1</smiles>

Compound 7n was prepared according to the general procedure GP-1 on a 0.2 mmol scale and was obtained after silica column chromatography using $n$-hexane : EtOAc $(40: 1 \rightarrow 9: 1) ; 61 \%$, (34.6 mg); colorless oil.

${ }^{1}$ H NMR $(600 \mathrm{MHz}$, Chloroform- $d)$ : $\delta=8.08-7.91(\mathrm{~m}, 2 \mathrm{H}), 7.65-7.56(\mathrm{~m}, 2 \mathrm{H}), 7.51-7.36$ $(\mathrm{m}, 3 \mathrm{H}), 7.30-7.22(\mathrm{~m}, 2 \mathrm{H}), 6.16(\mathrm{~s}, 1 \mathrm{H}), 3.75(\mathrm{~s}, 3 \mathrm{H}), 2.42(\mathrm{~s}, 3 \mathrm{H}) \mathrm{ppm}$.

${ }^{13}$ C NMR (151 MHz, Chloroform- $d$ ): $\delta=169.3,165.9,144.2,134.0,130.0,129.2,129.1,128.8$, 127.6, 126.4, 74.7, 52.6, $21.7 \mathrm{ppm}$.

HRMS (ESI): $m / z$ : $[\mathrm{M}+\mathrm{K}]^{+}$Calcd. for $\mathrm{C}_{17} \mathrm{H}_{16} \mathrm{KO}_{4}{ }^{+}: 323.0680$; Found: 323.0679 .

IR (KBr): 3345, 2158, 2115, 1926, 1750, 1714, 1639, 1498, 1437, 1410, 1348, 1330, 1283, $1252,1212,1172,1096,1030,965,897,838,751,694 \mathrm{~cm}^{-1}$. 
<smiles>COC(=O)C(OC(=O)c1ccc(C(C)(C)C)cc1)c1ccccc1</smiles>

Compound 7o was prepared according to the general procedure GP-1 on a 0.2 mmol scale and was obtained after silica column chromatography using $n$-hexane : EtOAc $(40: 1 \rightarrow 9: 1) ; 69 \%$, (45.1 mg); colorless oil.

${ }^{1}$ H NMR $(600 \mathrm{MHz}$, Chloroform- $d): \delta=8.10-7.93(\mathrm{~m}, 2 \mathrm{H}), 7.62-7.46(\mathrm{~m}, 2 \mathrm{H}), 7.46-7.37$ $(\mathrm{m}, 2 \mathrm{H}), 7.37-7.27(\mathrm{~m}, 3 \mathrm{H}), 6.09(\mathrm{~s}, 1 \mathrm{H}), 3.67(\mathrm{~s}, 3 \mathrm{H}), 1.26(\mathrm{~s}, 9 \mathrm{H}) \mathrm{ppm}$.

${ }^{13}$ C NMR (151 MHz, Chloroform- $d$ ): $\delta=169.3,165.8,157.2,134.1,129.8,129.2,128.8,127.6$, 126.4, 125.4, 74.7, 52.6, 35.1, $31.1 \mathrm{ppm}$.

HRMS (ESI): $m / z$ : $[\mathrm{M}+\mathrm{K}]^{+}$Calcd. for $\mathrm{C}_{20} \mathrm{H}_{22} \mathrm{KO}_{4}{ }^{+}: 365.1149$; Found: 365.1140 .

IR (KBr): 3428, 3036, 2960, 2871, 2711, 2326, 2170, 2078, 2000, 1927, 1811, 1755, 1721, $1608,1497,1457,1408,1352,1260,1215,1181,1108,1020,970,853,774,733,699 \mathrm{~cm}^{-1}$.

\section{2-Methoxy-2-oxo-1-phenylethyl 4-fluorobenzoate (7p)}<smiles>COC(=O)C(OC(=O)c1ccc(F)cc1)c1ccccc1</smiles>

Compound 7p was prepared according to the general procedure GP-1 on a $0.2 \mathrm{mmol}$ scale and was obtained after silica column chromatography using $n$-hexane : EtOAc $(40: 1 \rightarrow 9: 1) ; 75 \%$, (43.3 mg); colorless oil.

${ }^{1}$ H NMR $(600 \mathrm{MHz}$, Chloroform- $d$ ): $\delta=\delta 8.22-8.07(\mathrm{~m}, 2 \mathrm{H}), 7.61-7.51(\mathrm{~m}, 2 \mathrm{H}), 7.46-$ $7.39(\mathrm{~m}, 3 \mathrm{H}), 7.18-7.11(\mathrm{~m}, 2 \mathrm{H}), 6.15(\mathrm{~s}, 1 \mathrm{H}), 3.76(\mathrm{~s}, 3 \mathrm{H}) \mathrm{ppm}$.

${ }^{13}$ C NMR (151 MHz, Chloroform- $d$ ): $\delta=169.2,166.1\left(\mathrm{~d}, \mathrm{C}-\mathrm{F},{ }^{1} J_{\mathrm{C}-\mathrm{F}}=254.7 \mathrm{~Hz}\right), 164.8,133.8$, $132.5\left(\mathrm{~d}, \mathrm{C}-\mathrm{F},{ }^{3} J_{\mathrm{C}-\mathrm{F}}=9.5 \mathrm{~Hz}\right), 129.3,128.9,127.6,125.4\left(\mathrm{~d}, \mathrm{C}-\mathrm{F},{ }^{4} J_{\mathrm{C}-\mathrm{F}}=3.1 \mathrm{~Hz}\right), 115.6(\mathrm{~d}, \mathrm{C}-$ $\left.\mathrm{F},{ }^{2} J_{\mathrm{C}-\mathrm{F}}=22.1 \mathrm{~Hz}\right), 74.9,52.7 \mathrm{ppm}$.

${ }^{19}$ F NMR (565 MHz, Chloroform- $d$ ): $\delta=-95.38--117.71(\mathrm{~m}) \mathrm{ppm}$.

HRMS (ESI): $m / z$ : $[\mathrm{M}+\mathrm{K}]^{+}$Calcd. for $\mathrm{C}_{16} \mathrm{H}_{13} \mathrm{FKO}_{4}{ }^{+}: 327.0429$; Found: 327.0427.

IR (KBr): 3447, 3070, 3036, 2956, 2847, 2321, 2176, 2075, 1993, 1917, 1812, 1753, 1724, $1602,1505,1438,1413,1348,1255,1219,1179,1154,1103,1032,969,916,853,803,765$, $734,694 \mathrm{~cm}^{-1}$. 
<smiles>COC(=O)C(OC(=O)c1cccc(C)c1)c1ccccc1</smiles>

Compound 7q was prepared according to the general procedure GP-1 on a 0.2 mmol scale and was obtained after silica column chromatography using $n$-hexane : EtOAc $(40: 1 \rightarrow 9: 1) ; 75 \%$, (42.8 mg); colorless oil.

${ }^{1}$ H NMR (600 MHz, Chloroform- $\left.d\right): \delta=8.01-7.77(\mathrm{~m}, 2 \mathrm{H}), 7.58-7.48(\mathrm{~m}, 2 \mathrm{H}), 7.41-7.22$ $(\mathrm{m}, 5 \mathrm{H}), 6.09(\mathrm{~s}, 1 \mathrm{H}), 3.67(\mathrm{~s}, 3 \mathrm{H}), 2.33(\mathrm{~s}, 3 \mathrm{H}) \mathrm{ppm}$.

${ }^{13}$ C NMR (151 MHz, Chloroform- $d$ ): $\delta=169.3$, 166.0, 138.2, 134.2, 134.0, 130.4, 129.2, 129.1, $128.8,128.3,127.6,127.1,74.8,52.6,21.2 \mathrm{ppm}$.

HRMS (ESI): $m / z$ : $[\mathrm{M}+\mathrm{K}]^{+}$Calcd. for $\mathrm{C}_{17} \mathrm{H}_{16} \mathrm{KO}_{4}{ }^{+}: 323.0680$; Found: 323.0673 .

IR (KBr): 3859, 3427, 3034, 2954, 2666, 2323, 2160, 2091, 1992, 1016, 1754, 1721, 1591, $1492,1437,1349,1265,1191,1104,1082,1036,972,926,851,804,744,695 \mathrm{~cm}^{-1}$.

\section{2-Methoxy-2-oxo-1-phenylethyl 3-bromobenzoate (7r)}<smiles>COC(=O)C(OC(=O)c1cccc(Br)c1)c1ccccc1</smiles>

Compound 7r was prepared according to the general procedure GP-1 on a $0.2 \mathrm{mmol}$ scale and was obtained after silica column chromatography using $n$-hexane : EtOAc $(40: 1 \rightarrow 9: 1) ; 82 \%$, $(56.9 \mathrm{mg})$; colorless oil.

${ }^{1}$ H NMR (600 MHz, Chloroform- $d$ ): $\delta=8.24(\mathrm{t}, J=1.8 \mathrm{~Hz}, 1 \mathrm{H}), 8.10-7.97(\mathrm{~m}, 1 \mathrm{H}), 7.72$ (ddd, $J=7.9,2.1,1.1 \mathrm{~Hz}, 1 \mathrm{H}), 7.60-7.54(\mathrm{~m}, 2 \mathrm{H}), 7.48-7.39(\mathrm{~m}, 3 \mathrm{H}), 7.34(\mathrm{t}, J=7.9 \mathrm{~Hz}$, $1 \mathrm{H}), 6.16(\mathrm{~s}, 1 \mathrm{H}), 3.76(\mathrm{~s}, 3 \mathrm{H}) \mathrm{ppm}$.

${ }^{13}$ C NMR (151 MHz, Chloroform- $d$ ): $\delta=169.0,164.5,136.4,133.6,132.8,131.1,130.0,129.4$, $128.9,128.5,127.7,122.5,75.1,52.7 \mathrm{ppm}$.

HRMS (ESI): $m / z:[\mathrm{M}+\mathrm{K}]^{+}$Calcd. for $\mathrm{C}_{16} \mathrm{H}_{13} \mathrm{BrKO}_{4}{ }^{+}: 386.9628$; Found: 386.9628.

IR (KBr): 3449, 3068, 2954, 2159, 2016, 1726, 1570, 1495, 1429, 1349, 1284, 1246, 1215 , $1173,1034,967,841,806,787,742,696 \mathrm{~cm}^{-1}$. 
<smiles>COC(=O)C(OC(=O)c1cccc(OC)c1)c1ccccc1</smiles>

Compound 7s was prepared according to the general procedure GP-1 on a 0.2 mmol scale and was obtained after silica column chromatography using $n$-hexane : EtOAc (40:1 $\rightarrow$ 9:1); 54\%, (32.5 mg); colorless oil.

${ }^{1}$ H NMR $(600 \mathrm{MHz}$, Chloroform- $d): \delta=7.74(\mathrm{dt}, J=7.7,1.3 \mathrm{~Hz}, 1 \mathrm{H}), 7.65-7.61(\mathrm{~m}, 1 \mathrm{H})$, $7.61-7.53(\mathrm{~m}, 2 \mathrm{H}), 7.47-7.39(\mathrm{~m}, 3 \mathrm{H}), 7.37(\mathrm{t}, J=7.9 \mathrm{~Hz}, 1 \mathrm{H}), 7.21-7.11(\mathrm{~m}, 1 \mathrm{H}), 6.16(\mathrm{~s}$, $1 \mathrm{H}), 3.85(\mathrm{~s}, 3 \mathrm{H}), 3.76(\mathrm{~s}, 3 \mathrm{H}) \mathrm{ppm}$.

${ }^{13}$ C NMR (151 MHz, Chloroform- $d$ ): $\delta=169.2,165.7,159.5,133.9,130.4,129.5,129.3,128.8$, 127.6, 122.4, 119.9, 114.4, 74.9, 55.4, $52.6 \mathrm{ppm}$.

HRMS (ESI): $m / z$ : $[\mathrm{M}+\mathrm{K}]^{+}$Calcd. for $\mathrm{C}_{17} \mathrm{H}_{16} \mathrm{KO}_{5}{ }^{+}$: 339.0629; Found: 339.0628 .

IR (KBr): 3442, 3067, 3006, 2954, 2839, 2656, 2327, 2193, 2092, 1993, 1903, 1753, 1722, $1590,1487,1435,1268,1215,1176,1102,1039,972,921,874,800,753,695 \mathrm{~cm}^{-1}$.

\section{2-Methoxy-2-oxo-1-phenylethyl 2-methylbenzoate (7t)}<smiles>COC(=O)C(OC(=O)c1ccccc1C)c1ccccc1</smiles>

Compound $7 \mathbf{t}$ was prepared according to the general procedure GP-1 on a $0.2 \mathrm{mmol}$ scale and was obtained after silica column chromatography using $n$-hexane : EtOAc (40:1 $\rightarrow$ 9:1); 70\%, (40.0 mg); colorless oil.

${ }^{1}$ H NMR $(600 \mathrm{MHz}$, Chloroform- $d)$ : $\delta=8.13-8.04(\mathrm{~m}, 1 \mathrm{H}), 7.66-7.58(\mathrm{~m}, 2 \mathrm{H}), 7.53-7.38$ (m, 4H), $7.34-7.22(\mathrm{~m}, 2 \mathrm{H}), 6.19(\mathrm{~s}, 1 \mathrm{H}), 3.79(\mathrm{~s}, 3 \mathrm{H}), 2.67(\mathrm{~s}, 3 \mathrm{H}) \mathrm{ppm}$.

${ }^{13}$ C NMR (151 MHz, Chloroform- $d$ ): $\delta=169.4,166.7,140.7,134.0,132.5,131.7,131.0,129.2$, 128.8, 128.6, 127.7, 125.8, 74.8, 52.6, $21.7 \mathrm{ppm}$.

HRMS (ESI): $\mathrm{m} / z$ : $[\mathrm{M}+\mathrm{K}]^{+}$Calcd. for $\mathrm{C}_{17} \mathrm{H}_{16} \mathrm{KO}_{4}{ }^{+}: 323.0680$; Found: 323.0671 .

IR (KBr): 3865, 3443, 3065, 3032, 2955, 2660, 2322, 2086, 1994, 1910, 1753, 1721, 1601, 1577, 1492, 1438, 1383, 1348, 1245, 1216, 1177, 1136, 1076, 1030, 968, 914, 842, 738, 697 $\mathrm{cm}^{-1}$. 
<smiles>COC(=O)C(OC(=O)c1ccccc1F)c1ccccc1</smiles>

Compound $7 \mathbf{u}$ was prepared according to the general procedure GP-1 on a $0.2 \mathrm{mmol}$ scale and was obtained after silica column chromatography using $n$-hexane : EtOAc $(40: 1 \rightarrow 9: 1)$; 66\%, (38.1 mg); colorless oil.

${ }^{1}$ H NMR $(600 \mathrm{MHz}$, Chloroform- $d): \delta=8.11-7.97(\mathrm{~m}, 1 \mathrm{H}), 7.66-7.58(\mathrm{~m}, 2 \mathrm{H}), 7.56-7.52$ $(\mathrm{m}, 1 \mathrm{H}), 7.47-7.37(\mathrm{~m}, 3 \mathrm{H}), 7.23(\mathrm{t}, J=7.6 \mathrm{~Hz}, 1 \mathrm{H}), 7.19-7.11(\mathrm{~m}, 1 \mathrm{H}), 6.18(\mathrm{~s}, 1 \mathrm{H}), 3.76$ (s, 3H) ppm.

${ }^{13}$ C NMR (151 MHz, Chloroform- $\left.d\right): \delta=169.0,163.4\left(\mathrm{~d}, \mathrm{C}-\mathrm{F},{ }^{4} J_{\mathrm{C}-\mathrm{F}}=3.8 \mathrm{~Hz}\right), 162.3(\mathrm{~d}, \mathrm{C}-\mathrm{F}$, $\left.{ }^{1} J_{\mathrm{C}-\mathrm{F}}=261.6 \mathrm{~Hz}\right), 135.1\left(\mathrm{~d}, \mathrm{C}-\mathrm{F},{ }^{3} J_{\mathrm{C}-\mathrm{F}}=9.2 \mathrm{~Hz}\right), 133.6,132.4,129.2,128.8,127.5,124.0(\mathrm{~d}$, $\left.\mathrm{C}-\mathrm{F},{ }^{4} J_{\mathrm{C}-\mathrm{F}}=3.9 \mathrm{~Hz}\right), 117.7\left(\mathrm{~d}, \mathrm{C}-\mathrm{F},{ }^{3} J_{\mathrm{C}-\mathrm{F}}=9.2 \mathrm{~Hz}\right), 117.1\left(\mathrm{~d}, \mathrm{C}-\mathrm{F},{ }^{2} J_{\mathrm{C}-\mathrm{F}}=22.2 \mathrm{~Hz}\right), 75.0,52.7$ ppm.

${ }^{19}$ F NMR (565 MHz, Chloroform- $d$ ): $\delta=-99.86--116.54(\mathrm{~m}) \mathrm{ppm}$.

HRMS (ESI): $m / z$ : $[\mathrm{M}+\mathrm{K}]^{+}$Calcd. for $\mathrm{C}_{16} \mathrm{H}_{13} \mathrm{FKO}_{4}{ }^{+}: 327.0429$; Found: 327.0421.

IR (KBr): 3858, 3451, 3036, 2955, 2850, 2686, 2324, 2090, 1999, 1913, 1727, 1612, 1584, $1489,1454,1349,1294,1245,1218,1179,1123,1080,1032,966,916,846,808,755,696$, $658 \mathrm{~cm}^{-1}$.

\section{2-Methoxy-2-oxo-1-phenylethyl 2-bromobenzoate (7v)}<smiles>COC(=O)C(OC(=O)c1ccccc1Br)c1ccccc1</smiles>

Compound $7 \mathbf{v}$ was prepared according to the general procedure GP-1 on a $0.2 \mathrm{mmol}$ scale and was obtained after silica column chromatography using $n$-hexane : EtOAc $(40: 1 \rightarrow 9: 1) ; 55 \%$, (38.3 mg); colorless oil.

${ }^{1}$ H NMR $(600 \mathrm{MHz}$, Chloroform- $d)$ : $\delta=8.02-7.95(\mathrm{~m}, 1 \mathrm{H}), 7.71-7.62(\mathrm{~m}, 1 \mathrm{H}), 7.60-7.51$ $(\mathrm{m}, 2 \mathrm{H}), 7.45-7.29(\mathrm{~m}, 5 \mathrm{H}), 6.19(\mathrm{~s}, 1 \mathrm{H}), 3.77$ (s, 3H) ppm.

${ }^{13}$ C NMR (151 MHz, Chloroform- $d$ ): $\delta=169.0,165.1,134.5,133.4,133.1,132.0,130.8,129.3$, $128.8,127.7,127.2,122.2,75.3,52.7 \mathrm{ppm}$.

HRMS (ESI): $m / z$ : $[\mathrm{M}+\mathrm{K}]^{+}$Calcd. for $\mathrm{C}_{16} \mathrm{H}_{13} \mathrm{BrKO}_{4}{ }^{+}: 386.9628$; Found: 386.9647.

IR (KBr): 3456, 3066, 3034, 2954, 2847, 2329, 2161, 2084, 1736, 1588, 1495, 1434, 1346, $1243,1216,1177,1104,1026,966,916,833,742,696 \mathrm{~cm}^{-1}$. 
<smiles>COC(=O)C(OC(=O)c1cc(C(F)(F)F)cc(C(F)(F)F)c1)c1ccccc1</smiles>

Compound $7 \mathbf{w}$ was prepared according to the general procedure GP-1 on a 0.2 mmol scale and was obtained after silica column chromatography using $n$-hexane : EtOAc (40:1 $\rightarrow$ 9:1); 53\%, (43.2 mg); colorless oil.

${ }^{1}$ H NMR $(600 \mathrm{MHz}$, Chloroform- $d)$ : $\delta=8.47(\mathrm{~s}, 2 \mathrm{H}), 8.03(\mathrm{~s}, 1 \mathrm{H}), 7.61-7.45(\mathrm{~m}, 2 \mathrm{H}), 7.45$ $-7.33(\mathrm{~m}, 3 \mathrm{H}), 6.14(\mathrm{~s}, 1 \mathrm{H}), 3.71(\mathrm{~s}, 3 \mathrm{H}) \mathrm{ppm}$

${ }^{13}$ C NMR (151 MHz, Chloroform- $d$ ): $\delta=168.6,163.3,133.0,132.3$ (q, C-F, ${ }^{2} J_{\mathrm{C}-\mathrm{F}}=34.2 \mathrm{~Hz}$ ), 131.4, 130.1 - 129.9 (m, C-F), 129.7, 129.1, $127.8,127.2$ - 126.5 (m, C-F), 122.7 (q, C-F, ${ }^{1} J_{\mathrm{C}-}$ $\mathrm{F}=273.1 \mathrm{~Hz}), 75.7,52.9 \mathrm{ppm}$.

${ }^{19}$ F NMR (565 MHz, Chloroform- $d$ ): $\delta=-62.97$ ppm.

HRMS (ESI): $m / z:[\mathrm{M}+\mathrm{K}]^{+}$Calcd. for $\mathrm{C}_{18} \mathrm{H}_{12} \mathrm{~F}_{6} \mathrm{KO}_{4}{ }^{+}: 445.0271$; Found: 445.0261 .

IR (KBr): 3070, 2959, 1737, 1622, 1498, 1440, 1389, 1348, 1276, 1241, 1176, 1132, 1032, $969,912,846,765,735,697 \mathrm{~cm}^{-1}$.

\section{2-Methoxy-2-oxo-1-phenylethyl 2-naphthoate (7x)}<smiles>COC(=O)C(OC(=O)c1ccc2ccccc2c1)c1ccccc1</smiles>

Compound 7x was prepared according to the general procedure GP-1 on a $0.2 \mathrm{mmol}$ scale and was obtained after silica column chromatography using $n$-hexane : EtOAc (40:1 $\rightarrow$ 9:1); 65\%, (41.8 mg); colorless oil.

${ }^{1}$ H NMR $(600 \mathrm{MHz}$, Chloroform- $d$ ): $\delta=8.73(\mathrm{~s}, 1 \mathrm{H}), 8.16(\mathrm{dd}, J=8.6,1.7 \mathrm{~Hz}, 1 \mathrm{H}), 8.04-$ $7.98(\mathrm{~m}, 1 \mathrm{H}), 7.98-7.90(\mathrm{~m}, 2 \mathrm{H}), 7.72-7.64(\mathrm{~m}, 2 \mathrm{H}), 7.66-7.59(\mathrm{~m}, 1 \mathrm{H}), 7.61-7.54(\mathrm{~m}$, $1 \mathrm{H}), 7.54-7.45(\mathrm{~m}, 3 \mathrm{H}), 6.28(\mathrm{~s}, 1 \mathrm{H}), 3.81(\mathrm{~s}, 3 \mathrm{H}) \mathrm{ppm}$.

${ }^{13}$ C NMR (151 MHz, Chloroform- $d$ ): $\delta=169.3,166.0,135.8,134.0,132.4,131.7,129.4,129.3$, $128.9,128.5,128.3,127.8,127.7,126.7,126.4,125.3,75.0,52.7 \mathrm{ppm}$.

HRMS (ESI): $m / z$ : $[\mathrm{M}+\mathrm{K}]^{+}$Calcd. for $\mathrm{C}_{20} \mathrm{H}_{16} \mathrm{KO}_{4}{ }^{+}: 359.0680$; Found: 359.0672 .

IR (KBr): 3424, 3061, 2954, 2258, 2164, 1934, 1753, 1718, 1630, 1598, 1498, 1438, 1353, $1265,1218,1189,1131,1091,1032,962,911,866,827,772,731,696 \mathrm{~cm}^{-1}$. 
<smiles>COC(=O)C(OC(=O)c1cccc2ccccc12)c1ccccc1</smiles>

Compound $7 \mathbf{y}$ was prepared according to the general procedure GP-1 on a $0.2 \mathrm{mmol}$ scale and was obtained after silica column chromatography using $n$-hexane : EtOAc $(40: 1 \rightarrow 9: 1) ; 63 \%$, (40.6 mg); colorless oil.

${ }^{1} \mathbf{H}$ NMR $(600 \mathrm{MHz}$, Chloroform- $d)$ : $\delta={ }^{1} \mathrm{H}$ NMR $\left(600 \mathrm{MHz}, \mathrm{CDCl}_{3}\right) \delta 9.02-8.85(\mathrm{~m}, 1 \mathrm{H})$, $8.36(\mathrm{dd}, J=7.3,1.3 \mathrm{~Hz}, 1 \mathrm{H}), 8.09-8.00(\mathrm{~m}, 1 \mathrm{H}), 7.93-7.83(\mathrm{~m}, 1 \mathrm{H}), 7.68-7.60(\mathrm{~m}, 3 \mathrm{H})$, $7.58-7.49(\mathrm{~m}, 2 \mathrm{H}), 7.46-7.37(\mathrm{~m}, 3 \mathrm{H}), 6.30(\mathrm{~s}, 1 \mathrm{H}), 3.80(\mathrm{~s}, 3 \mathrm{H}) \mathrm{ppm}$

${ }^{13}$ C NMR (151 MHz, Chloroform- $d$ ): $\delta=169.5,166.7,133.9,133.8,131.4,130.8,129.3,128.9$, $128.5,127.9,127.7,126.3,126.0,125.7,124.5,74.9,52.7 \mathrm{ppm}$.

HRMS (ESI): $m / z:[\mathrm{M}+\mathrm{Na}]^{+}$Calcd. for $\mathrm{C}_{20} \mathrm{H}_{16} \mathrm{NaO}_{4}{ }^{+}: 343.0940$; Found: 343.0951.

IR (KBr): 3421, 3056, 2953, 2846, 2662, 2323, 1900, 1752, 1717, 1588, 1508, 1438, 1397 , 1346, 1276, 1238, 1216, 1189, 1128, 1076, 1044, 1001, 913, 853, 779, 734, 696, $658 \mathrm{~cm}^{-1}$.

\section{2-Methoxy-2-oxo-1-phenylethyl 2,3,4,5,6-pentafluorobenzoate (7z)}<smiles>COC(=O)C(OC(=O)c1c(F)c(F)c(F)c(F)c1F)c1ccccc1</smiles>

Compound $7 \mathbf{z}$ was prepared according to the general procedure GP-1 on a 0.2 mmol scale and was obtained after silica column chromatography using $n$-hexane : EtOAc $(40: 1 \rightarrow 9: 1) ; 69 \%$, (49.6 mg); colorless oil.

${ }^{1}$ H NMR (600 MHz, Chloroform- $d$ ): $\delta=7.58-7.42(\mathrm{~m}, 2 \mathrm{H}), 7.42-7.29(\mathrm{~m}, 3 \mathrm{H}), 6.12(\mathrm{~s}, 1 \mathrm{H})$, $3.70(\mathrm{~s}, 3 \mathrm{H}) \mathrm{ppm}$.

${ }^{13}$ C NMR (151 MHz, Chloroform- $d$ ): $\delta=168.1,158.4,147.4-144.8$ (m, C-F), $144.9-142.5$ (m, C-F), 139.5 - 136.3 (m, C-F), 132.7, 129.6, 128.9, 127.6, 108.1 - 105.6 (m, C-F), 76.16, $52.9 \mathrm{ppm}$.

${ }^{19}$ F NMR (565 MHz, Chloroform- $d$ ): $\delta=-135.94--137.41$ (m, 3F), -145.77 - -148.77 (m, 1F), $-159.39--161.87(\mathrm{~m}, 1 \mathrm{~F}) \mathrm{ppm}$.

HRMS (ESI): $m / z$ : $[\mathrm{M}+\mathrm{Na}]^{+}$Calcd. for $\mathrm{C}_{16} \mathrm{H}_{9} \mathrm{~F}_{5} \mathrm{NaO}_{4}{ }^{+}: 383.0313$; Found: 383.0301.

IR (KBr): 3482, 3036, 2959, 2851, 2667, 2325, 2085, 1916, 1742, 1651, 1499, 1428, 1331, $1274,1214,1177,1104,1000,872,809,757,734,696 \mathrm{~cm}^{-1}$. 
<smiles>COC(=O)C(OC(=O)c1cccs1)c1ccccc1</smiles>

Compound 7aa was prepared according to the general procedure GP- 1 on a 0.2 mmol scale and was obtained after silica column chromatography using $n$-hexane : EtOAc $(40: 1 \rightarrow 9: 1)$; 48\%, (26.3 mg); colorless oil.

${ }^{1}$ H NMR $(600 \mathrm{MHz}$, Chloroform- $d): \delta=8.00-7.87(\mathrm{~m}, 1 \mathrm{H}), 7.69-7.58(\mathrm{~m}, 1 \mathrm{H}), 7.59-7.49$ $(\mathrm{m}, 2 \mathrm{H}), 7.49-7.32(\mathrm{~m}, 3 \mathrm{H}), 7.19-7.02(\mathrm{~m}, 1 \mathrm{H}), 6.14(\mathrm{~s}, 1 \mathrm{H}), 3.75(\mathrm{~d}, J=0.8 \mathrm{~Hz}, 3 \mathrm{H}) \mathrm{ppm}$. ${ }^{13}$ C NMR (151 MHz, Chloroform- $d$ ): $\delta=169.1,161.4,134.4,133.7,133.2,132.5,129.3,128.8$, $127.8,127.6,74.8,52.7 \mathrm{ppm}$.

HRMS (ESI): $m / z$ : $[\mathrm{M}+\mathrm{Na}]^{+}$Calcd. for $\mathrm{C}_{14} \mathrm{H}_{12} \mathrm{NaO}_{4} \mathrm{~S}^{+}: 299.0348$; Found: 299.0342.

IR (KBr): 3415, 3101, 3034, 2954, 2846, 2667, 2324, 2083, 1993, 1914, 1752, 1710, 1586, 1523, 1496, 1415, 1360, 1254, 1216, 1177, 1086, 1019, 966, 917, 858, 810, 746, $697 \mathrm{~cm}^{-1}$.

\section{2,2,2-Trifluoro-1-phenylethyl benzoate (8)}<smiles>O=C(OC(c1ccccc1)C(F)(F)F)c1ccccc1</smiles>

Compound 8 was prepared according to the general procedure GP- 1 on a 0.2 mmol scale and was obtained after silica column chromatography using $n$-hexane : EtOAc $(40: 1 \rightarrow 9: 1) ; 45.7 \%$, (25.6 mg); colorless oil.

${ }^{1}$ H NMR (600 MHz, Chloroform- $\left.d\right): \delta=8.19-7.90(\mathrm{~m}, 2 \mathrm{H}), 7.71-7.62(\mathrm{~m}, 1 \mathrm{H}), 7.62-7.54$ $(\mathrm{m}, 2 \mathrm{H}), 7.52(\mathrm{t}, J=7.8 \mathrm{~Hz}, 2 \mathrm{H}), 7.49-7.42(\mathrm{~m}, 3 \mathrm{H}), 6.40(\mathrm{q}, J=6.9 \mathrm{~Hz}, 1 \mathrm{H}) \mathrm{ppm}$.

${ }^{13}$ C NMR (151 MHz, Chloroform- $\left.d\right)$ : $\delta=164.3,133.8,131.3,130.0,129.9,128.76,128.74$, 128.6, 128.0, $123.3(\mathrm{q}, J=280.4 \mathrm{~Hz}), 72.4(\mathrm{q}, J=33.1 \mathrm{~Hz}) \mathrm{ppm}$.

${ }^{19}$ F NMR (565 MHz, Chloroform- $d$ ): $\delta=-75.82(\mathrm{~d}, J=7.0 \mathrm{~Hz}) \mathrm{ppm}$.

HRMS (ESI): $m / z:[\mathrm{M}+\mathrm{K}]^{+}$Calcd. for $\mathrm{C}_{15} \mathrm{H}_{11} \mathrm{~F}_{3} \mathrm{KO}_{2}{ }^{+}: 319.0342$; Found: 319.0340.

IR (KBr): 3458, 3068, 3040, 2962, 2704, 2325, 2187, 2088, 1994, 1910, 1736, 1600, 1496, $1453,1350,1314,1255,1179,1131,1094,1026,902,871,803,758,703 \mathrm{~cm}^{-1}$. 


\section{Benzhydryl benzoate (9)}<smiles>O=C(OC(c1ccccc1)c1ccccc1)c1ccccc1</smiles>

Compound 9 was prepared according to the general procedure GP-1 on a $0.2 \mathrm{mmol}$ scale and was obtained after silica column chromatography using $n$-hexane : EtOAc $(40: 1 \rightarrow 9: 1) ; 79 \%$, (45.4 mg); colorless crystals.

${ }^{1}$ H NMR (400 MHz, Chloroform- $d$ ): $\delta=8.24-8.01(\mathrm{~m}, 2 \mathrm{H}), 7.68-7.55(\mathrm{~m}, 1 \mathrm{H}), 7.49-7.42$ (m, 6H), $7.42-7.24(\mathrm{~m}, 6 \mathrm{H}), 7.14(\mathrm{~s}, 1 \mathrm{H}) \mathrm{ppm}$.

${ }^{13}$ C NMR (101 MHz, Chloroform- $d$ ): $\delta=165.5,140.2,133.1,129.7,128.5,128.4,127.9,127.1$, $77.4 \mathrm{ppm}$.

HRMS (ESI): $m / z$ : [M $+\mathrm{K}]^{+}$Calcd. for $\mathrm{C}_{20} \mathrm{H}_{16} \mathrm{KO}_{2}{ }^{+}: 327.0791$; Found: 327.0780 .

IR (KBr): 3414, 3058, 3013, 2927, 2328, 2189, 2084, 1909, 1785, 1710, 1592, 1493, 1451, $1316,1262,1177,1104,1023,968,932,898,847,753,698 \mathrm{~cm}^{-1}$.

\section{2-Ethoxy-2-oxoethyl benzoate (10)}<smiles>CCOC(=O)COC(=O)c1ccccc1</smiles>

Compound 10 was prepared according to the general procedure GP-1 on a $0.2 \mathrm{mmol}$ scale and was obtained after silica column chromatography using $n$-hexane : EtOAc $(40: 1 \rightarrow 9: 1) ; 35 \%$, (14.5 mg); colorless oil.

${ }^{1}$ H NMR (600 MHz, Chloroform- $d$ ): $\delta=8.23-8.07$ (m, 2H), $7.68-7.53(\mathrm{~m}, 1 \mathrm{H}), 7.49$ (t, $J=$ $7.8 \mathrm{~Hz}, 2 \mathrm{H}), 4.87(\mathrm{~s}, 2 \mathrm{H}), 4.29(\mathrm{q}, J=7.1 \mathrm{~Hz}, 2 \mathrm{H}), 1.32(\mathrm{t}, J=7.1 \mathrm{~Hz}, 3 \mathrm{H}) \mathrm{ppm}$.

${ }^{13}$ C NMR (151 MHz, Chloroform- $d$ ): $\delta=167.8,166.0,133.4,129.9,129.2,128.4,61.4,61.2$, $14.1 \mathrm{ppm}$.

HRMS (ESI): $m / z$ : $[\mathrm{M}+\mathrm{K}]^{+}$Calcd. for $\mathrm{C}_{11} \mathrm{H}_{12} \mathrm{KO}_{4}{ }^{+}: 247.0367$; Found: 247.0363 .

IR (KBr): 3452, 2983, 2187, 2100, 1965, 1728, 1601, 1451, 1423, 1381, 1279, 1209, 1177 , $1115,1034,938,858,813,713 \mathrm{~cm}^{-1}$. 
<smiles>COC(=O)C(OC(=O)c1ccccc1-c1ccccc1C(=O)OC(C(=O)OC)c1ccccc1)c1ccccc1</smiles>

An oven-dried test tube was loaded with tropylium tetrafluoroborate $(20 \mathrm{~mol} \%)$ and the corresponding benzoic acid derivative (1.0 eq.; $0.2 \mathrm{mmol}$ ). The test tube was flushed and refilled with Ar for three times. Than diazoalkane (3.0 eq.; $0.6 \mathrm{mmol})$ dissolved in $1 \mathrm{~mL}$ dry, degassed DCM was added at once. The reaction mixture was stirred at room temperature until the orange color of the diazoalkane disappeared. The product was purified by silica column chromatography using $n$-hexane : EtOAc $(40: 1 \rightarrow 4: 1) ; 73 \%$, (78.5 mg); colorless solid; d.r. $\sim 1: 1.06$; atropisomer ratio $1: 1$.

${ }^{1}$ H NMR $(600 \mathrm{MHz}$, Chloroform- $d)$ : $\delta=8.15(\mathrm{~d}, J=1.4 \mathrm{~Hz}, 0.49 \mathrm{H}), 8.08(\mathrm{dd}, J=7.9,1.4 \mathrm{~Hz}$, $0.52 \mathrm{H}), 7.95(\mathrm{~d}, J=7.8 \mathrm{~Hz}, 1 \mathrm{H}), 7.59-7.45(\mathrm{~m}, 2 \mathrm{H}), 7.43-7.18(\mathrm{~m}, 12 \mathrm{H}), 7.17-7.12(\mathrm{~m}$, $1.01 \mathrm{H}), 7.08(\mathrm{~d}, J=7.3 \mathrm{~Hz}, 0.98 \mathrm{H}), 5.88(\mathrm{~d}, J=50.0 \mathrm{~Hz}, 0.93 \mathrm{H}), 5.88(\mathrm{~d}, J=50.0 \mathrm{~Hz}, 1 \mathrm{H})$, $3.65(\mathrm{~d}, J=31.4 \mathrm{~Hz}, 3.04 \mathrm{H}), 3.62(\mathrm{~d}, J=37.1 \mathrm{~Hz}, 2.92 \mathrm{H}) \mathrm{ppm}$.

${ }^{13}$ C NMR (151 MHz, Chloroform- $d$ ): $\delta=169.09,169.07,169.0,166.23,166.21,166.1,165.9$, 143.36, 143.31, 143.2, 143.1, 133.7, 133.6, 133.48, 133.40, 132.0, 131.9, 131.8, 131.7, 131.0, $130.6,130.5,130.4,130.3,129.0,128.9,128.8,128.58,128.53,128.5,128.4,128.3,128.2$, $128.0,127.77,127.70,127.67,127.65,127.29,127.25,127.23,74.9,74.7,74.7,52.47,52.46$, 52.42, $52.3 \mathrm{ppm}$.

HRMS (APCI): $m / z:[\mathrm{M}+\mathrm{H}]^{+}$Calcd. for $\mathrm{C}_{32} \mathrm{H}_{27} \mathrm{O}_{8}{ }^{+}:$539.1700; Found: 539.1706 .

IR (KBr): 3447, 3064, 3034, 2954, 2848, 2668, 2330, 2256, 2158, 1946, 1726, 1597, 1496, $1439,1348,1244,1216,1176,1122,1080,1046,967,910,835,730,699 \mathrm{~cm}^{-1}$.

\section{Methyl 2-acetoxy-2-phenylacetate (12a)}<smiles>COC(=O)C(OC(C)=O)c1ccccc1</smiles>

Compound 12a was prepared according to the general procedure GP- 1 on a 0.2 mmol scale and was obtained after silica column chromatography using $n$-hexane : EtOAc $(40: 1 \rightarrow 9: 1) ; 74 \%$, (30.6 mg); colorless oil.

${ }^{1}$ H NMR (400 MHz, Chloroform- $d$ ): $\delta=7.54-7.42$ (m, 2H), $7.42-7.29(\mathrm{~m}, 3 \mathrm{H}), 5.92(\mathrm{~s}, 1 \mathrm{H})$, $3.71(\mathrm{~s}, 3 \mathrm{H}), 2.18(\mathrm{~s}, 3 \mathrm{H}) \mathrm{ppm}$.

${ }^{13}$ C NMR (101 MHz, Chloroform- $d$ ): $\delta=170.3,169.3,133.7,129.2,128.7,127.6,74.4,52.6$, $20.6 \mathrm{ppm}$.

HRMS (ESI): $m / z$ : $[\mathrm{M}+\mathrm{Na}]^{+}$Calcd. for $\mathrm{C}_{11} \mathrm{H}_{12} \mathrm{NaO}_{4}{ }^{+}: 231.0627$; Found: 231.0625 .

IR (KBr): 3482, 3035, 2955, 2847, 2669, 2329, 2094, 1994, 1744, 1587, 1496, 1436, 1371, $1274,1220,1177,1053,977,928,853,780,737,697 \mathrm{~cm}^{-1}$. 
<smiles>CCC(=O)OC(C(=O)OC)c1ccccc1</smiles>

Compound 12b was prepared according to the general procedure GP- 1 on a 0.2 mmol scale and was obtained after silica column chromatography using $n$-hexane : EtOAc $(40: 1 \rightarrow 9: 1) ; 82 \%$, (36.6 mg); colorless oil.

${ }^{1}$ H NMR $(400 \mathrm{MHz}$, Chloroform- $d): \delta=7.55-7.41(\mathrm{~m}, 2 \mathrm{H}), 7.41-7.31(\mathrm{~m}, 3 \mathrm{H}), 5.93(\mathrm{~s}, 1 \mathrm{H})$, $3.70(\mathrm{~s}, 3 \mathrm{H}), 2.69-2.30(\mathrm{~m}, 2 \mathrm{H}), 1.18(\mathrm{t}, J=7.5 \mathrm{~Hz}, 3 \mathrm{H}) \mathrm{ppm}$.

${ }^{13}$ C NMR (101 MHz, Chloroform- $d$ ): $\delta=173.7,169.3,133.8,129.1,128.7,127.5,74.2,52.5$, 27.3, $8.8 \mathrm{ppm}$.

HRMS (ESI): $m / z$ : [M + Na $]^{+}$Calcd. for $\mathrm{C}_{12} \mathrm{H}_{14} \mathrm{NaO}_{4}{ }^{+}: 245.0784$; Found: 245.0781.

IR (KBr): 3483, 3035, 2984, 2951, 2672, 2326, 2174, 2086, 2011, 1743, 1589, 1497, 1437, $1351,1271,1214,1163,1084,1040,968,919,850,807,780,736,697 \mathrm{~cm}^{-1}$.

\section{2-Methoxy-2-oxo-1-phenylethyl hexanoate (12c)}<smiles>CCCCCC(=O)OC(C(=O)OC)c1ccccc1</smiles>

Compound 12c was prepared according to the general procedure GP- 1 on a 0.2 mmol scale and was obtained after silica column chromatography using $n$-hexane : EtOAc $(40: 1 \rightarrow 9: 1) ; 88 \%$, (46.3 mg); colorless oil.

${ }^{1}$ H NMR (400 MHz, Chloroform- $d$ ): $\delta=7.48-7.41(\mathrm{~m}, 2 \mathrm{H}), 7.43-7.33(\mathrm{~m}, 3 \mathrm{H}), 5.92(\mathrm{~s}, 1 \mathrm{H})$, $3.70(\mathrm{~s}, 3 \mathrm{H}), 2.61-2.29(\mathrm{~m}, 2 \mathrm{H}), 1.78-1.61(\mathrm{~m}, 2 \mathrm{H}), 1.40-1.26(\mathrm{~m}, 4 \mathrm{H}), 1.02-0.63(\mathrm{~m}$, 3H) $\mathrm{ppm}$.

${ }^{13}$ C NMR (101 MHz, Chloroform- $d$ ): $\delta=173.1,169.3,133.8,129.1,128.7,127.5,74.2,52.5$, $33.9,31.1,24.4,22.2,13.8 \mathrm{ppm}$.

HRMS (ESI): $m / z$ : $[\mathrm{M}+\mathrm{Na}]^{+}$Calcd. for $\mathrm{C}_{15} \mathrm{H}_{20} \mathrm{NaO}_{4}{ }^{+}: 287.1253$; Found: 287.1250 .

IR (KBr): 3482, 3067, 3025, 2954, 2865 2664, 2325, 2202, 2094, 1994, 1954, 1743, 1588, $1496,1438,1350,1271,1215,1158,1103,1046,979,945,913,851,735,697 \mathrm{~cm}^{-1}$. 
<smiles>CCCCCCC(=O)OC(C(=O)OC)c1ccccc1</smiles>

Compound 12d was prepared according to the general procedure GP- 1 on a 0.2 mmol scale and was obtained after silica column chromatography using $n$-hexane : EtOAc $(40: 1 \rightarrow 9: 1) ; 79 \%$, (46.0 mg); colorless oil.

${ }^{1}$ H NMR $(400 \mathrm{MHz}$, Chloroform- $d)$ : $\delta=7.48-7.41(\mathrm{~m}, 2 \mathrm{H}), 7.41-7.27(\mathrm{~m}, 3 \mathrm{H}), 5.92(\mathrm{~s}, 1 \mathrm{H})$, $3.70(\mathrm{~s}, 3 \mathrm{H}), 2.62-2.34(\mathrm{~m}, 2 \mathrm{H}), 1.67(\mathrm{p}, J=7.4 \mathrm{~Hz}, 2 \mathrm{H}), 1.49-1.22(\mathrm{~m}, 6 \mathrm{H}), 0.93-0.78(\mathrm{~m}$, 3H) ppm.

${ }^{13}$ C NMR (101 MHz, Chloroform- $d$ ): $\delta=173.1,169.3,133.9,129.1,128.7,127.5,74.2,52.5$, $33.9,31.3,28.6,24.7,22.4,13.9 \mathrm{ppm}$.

HRMS (ESI): $m / z:[\mathrm{M}+\mathrm{Na}]^{+}$Calcd. for $\mathrm{C}_{16} \mathrm{H}_{22} \mathrm{NaO}_{4}{ }^{+}: 301.1410$; Found: 301.1407 .

IR (KBr): 3484, 3035, 2930, 2861, 2663, 2327, 2173, 2094, 1990, 1744, 1589, 1496, 1455, $1349,1269,1214,1156,1105,1045,981,924,853,733,696 \mathrm{~cm}^{-1}$.

\section{Methyl 2-phenyl-2-(2-phenylacetoxy)acetate (12e)}

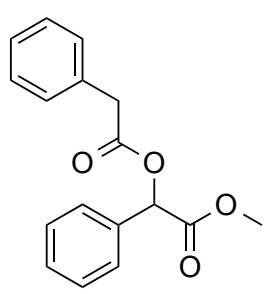

Compound 12e was prepared according to the general procedure GP- 1 on a $0.2 \mathrm{mmol}$ scale and was obtained after silica column chromatography using $n$-hexane : EtOAc $(40: 1 \rightarrow 9: 1) ; 65 \%$, (36.9 mg); colorless oil.

${ }^{1}$ H NMR $(400 \mathrm{MHz}$, Chloroform- $d): \delta=7.55-7.16(\mathrm{~m}, 10 \mathrm{H}), 5.94(\mathrm{~s}, 1 \mathrm{H}), 3.91-3.73(\mathrm{~m}$, $2 \mathrm{H}), 3.68(\mathrm{~s}, 3 \mathrm{H}) \mathrm{ppm}$.

${ }^{13}$ C NMR (101 MHz, Chloroform- $d$ ): $\delta=170.9,169.1,133.6,133.3,129.3,129.2,128.7,128.5$, $127.5,127.2,74.7,52.6,40.8 \mathrm{ppm}$.

HRMS (ESI): $m / z$ : $[\mathrm{M}+\mathrm{Na}]^{+}$Calcd. for $\mathrm{C}_{17} \mathrm{H}_{16} \mathrm{NaO}_{4}{ }^{+}: 307.0940$; Found: 307.0936 .

IR (KBr): 3484, 3064, 3033, 2954, 2846, 2670, 2327, 2094, 2958,1742, 1602, 1496, 1437 , $1353,1139,1079,1041,978,912,847,698 \mathrm{~cm}^{-1}$. 
<smiles>COC(=O)C(OC(=O)C(C)C)c1ccccc1</smiles>

Compound $\mathbf{1 2 f}$ was prepared according to the general procedure GP- 1 on a 0.2 mmol scale and was obtained after silica column chromatography using $n$-hexane : EtOAc $(40: 1 \rightarrow 9: 1) ; 71 \%$, (33.5 mg); colorless oil.

${ }^{1}$ H NMR (400 MHz, Chloroform- $d$ ): $\delta=7.59-7.41(\mathrm{~m}, 2 \mathrm{H}), 7.41-7.34(\mathrm{~m}, 3 \mathrm{H}), 5.90(\mathrm{~s}, 1 \mathrm{H})$, $3.70(\mathrm{~s}, 3 \mathrm{H}), 2.70(\mathrm{p}, J=7.0 \mathrm{~Hz}, 1 \mathrm{H}), 1.26(\mathrm{~d}, J=7.1 \mathrm{~Hz}, 3 \mathrm{H}), 1.20(\mathrm{~d}, J=7.0 \mathrm{~Hz}, 3 \mathrm{H}) \mathrm{ppm}$.

${ }^{13}$ C NMR (101 MHz, Chloroform- $d$ ): $\delta=176.3,169.3,133.9,129.1,128.7,127.5,74.1,52.5$, $33.7,18.8,18.6 \mathrm{ppm}$.

HRMS (ESI): $m / z:[\mathrm{M}+\mathrm{Na}]^{+}$Calcd. for $\mathrm{C}_{13} \mathrm{H}_{16} \mathrm{NaO}_{4}{ }^{+}: 259.0940$; Found: 259.0937.

IR (KBr): 3482, 3035, 2976, 2879, 2662, 2328, 2087, 1999, 1740, 1589, 1496, 1456, 1387 , $1346,1249,1215,1183,1039,975,935,885,831,734,696 \mathrm{~cm}^{-1}$.

\section{2-Methoxy-2-oxo-1-phenylethyl pivalate (12g)}<smiles>COC(=O)C(OC(=O)C(C)(C)C)c1ccccc1</smiles>

Compound $12 \mathrm{~g}$ was prepared according to the general procedure GP-1 on a $0.2 \mathrm{mmol}$ scale and was obtained after silica column chromatography using $n$-hexane : EtOAc $(40: 1 \rightarrow 9: 1) ; 60 \%$, (30.1 mg); colorless oil.

${ }^{1}$ H NMR (400 MHz, Chloroform- $d$ ): $\delta=7.52-7.43(\mathrm{~m}, 2 \mathrm{H}), 7.41-7.35$ (m, 3H), $5.88(\mathrm{~s}, 1 \mathrm{H})$, $3.69(\mathrm{~s}, 3 \mathrm{H}), 1.27(\mathrm{~s}, 9 \mathrm{H}) \mathrm{ppm}$.

${ }^{13}$ C NMR (101 MHz, Chloroform- $d$ ): $\delta=177.8,169.4,134.0,129.0,128.7,127.4,74.2,52.5$, 38.7, $27.0 \mathrm{ppm}$.

HRMS (ESI): $m / z:[\mathrm{M}+\mathrm{Na}]^{+}$Calcd. for $\mathrm{C}_{14} \mathrm{H}_{18} \mathrm{NaO}_{4}{ }^{+}: 273.1097$; Found: 273.1088.

IR (KBr): 3652, 2454, 3067, 3036, 2971, 2875, 2662, 2327, 2093, 1993, 1958, 1885, 1735, $1588,1539,1479,1455,1397,1356,1270,1215,1140,1083,1047,974,928,856,800,769$, $732,696 \mathrm{~cm}^{-1}$. 
<smiles>COC(=O)C(OC(=O)C1CCCC1)c1ccccc1</smiles>

Compound $12 \mathrm{~h}$ was prepared according to the general procedure GP- 1 on a 0.2 mmol scale and was obtained after silica column chromatography using $n$-hexane : EtOAc $(40: 1 \rightarrow 9: 1) ; 31.4 \%$, (62 mg); colorless oil.

${ }^{1}$ H NMR $(400 \mathrm{MHz}$, Chloroform- $d$ ): $\delta=7.52-7.40(\mathrm{~m}, 2 \mathrm{H}), 7.42-7.27(\mathrm{~m}, 3 \mathrm{H}), 5.91(\mathrm{~s}, 1 \mathrm{H})$, $3.70(\mathrm{~s}, 3 \mathrm{H}), 2.88(\mathrm{p}, J=7.9 \mathrm{~Hz}, 1 \mathrm{H}), 2.03-1.92(\mathrm{~m}, 2 \mathrm{H}), 1.92-1.80(\mathrm{~m}, 2 \mathrm{H}), 1.78-1.66(\mathrm{~m}$, 2H), $1.65-1.51(\mathrm{~m}, 2 \mathrm{H}) \mathrm{ppm}$.

${ }^{13}$ C NMR (101 MHz, Chloroform- $d$ ): $\delta=176.0,169.4,133.9,129.1,128.7,127.5,74.1,52.5$, 43.4, 30.0, 29.7, $25.8 \mathrm{ppm}$.

HRMS (ESI): $m / z:[\mathrm{M}+\mathrm{Na}]^{+}$Calcd. for $\mathrm{C}_{15} \mathrm{H}_{18} \mathrm{NaO}_{4}{ }^{+}: 285.1097$; Found: 285.1092 .

IR (KBr): 3462, 2956, 2872, 2328, 2172, 2091, 1991, 1738, 1588, 1495, 1448, 1347, 1268 , $1215,1146,1083,1046,986,896,850,734,696 \mathrm{~cm}^{-1}$.

\section{2-Methoxy-2-oxo-1-phenylethyl cyclohexanecarboxylate (12i)}<smiles>COC(=O)C(OC(=O)C1CCCCC1)c1ccccc1</smiles>

Compound 12i was prepared according to the general procedure GP- 1 on a $0.2 \mathrm{mmol}$ scale and was obtained after silica column chromatography using $n$-hexane : EtOAc $(40: 1 \rightarrow 9: 1) ; 69 \%$, (38.1 mg); colorless oil.

${ }^{1}$ H NMR $(400 \mathrm{MHz}$, Chloroform- $d): \delta=7.66-7.42(\mathrm{~m}, 2 \mathrm{H}), 7.42-7.18(\mathrm{~m}, 3 \mathrm{H}), 5.90(\mathrm{~s}, 1 \mathrm{H})$, $3.69(\mathrm{~s}, 3 \mathrm{H}), 2.52-2.30(\mathrm{~m}, 1 \mathrm{H}), 2.10-1.98(\mathrm{~m}, 1 \mathrm{H}), 1.92(\mathrm{~d}, J=13.2 \mathrm{~Hz}, 1 \mathrm{H}), 1.83-1.69$ $(\mathrm{m}, 2 \mathrm{H}), 1.64-1.58(\mathrm{~m}, 1 \mathrm{H}), 1.51(\mathrm{td}, J=13.6,12.8,6.0 \mathrm{~Hz}, 2 \mathrm{H}), 1.36-1.11(\mathrm{~m}, 3 \mathrm{H}) \mathrm{ppm}$.

${ }^{13}$ C NMR (101 MHz, Chloroform- $d$ ): $\delta=175.3,169.4,134.0,129.1,128.7,127.5,74.0,52.5$, 42.7, 28.9, 28.7, 25.6, 25.3, $25.2 \mathrm{ppm}$.

HRMS (ESI): $m / z$ : $[\mathrm{M}+\mathrm{Na}]^{+}$Calcd. for $\mathrm{C}_{16} \mathrm{H}_{20} \mathrm{NaO}_{4}{ }^{+}: 299.1253$; Found: 299.1250 .

IR (KBr): 3462, 3035, 2932, 2856, 2664, 2327, 2088, 1738, 1590, 1496, 1447, 1379, 1327, $1214,1161,1130,1046,975,941,893,844,734,696 \mathrm{~cm}^{-1}$. 
<smiles>COC(=O)C(OC(=O)C1CC1)c1ccccc1</smiles>

Compound $\mathbf{1 2} \mathbf{j}$ was prepared according to the general procedure GP- 1 on a $0.2 \mathrm{mmol}$ scale and was obtained after silica column chromatography using $n$-hexane : EtOAc $(40: 1 \rightarrow 9: 1) ; 63 \%$, (39.2 mg); colorless oil; d.r. $=1: 1.04$

${ }^{1}$ H NMR (400 MHz, Chloroform- $d$ ): $\delta=7.58-7.48(\mathrm{~m}, 2 \mathrm{H}), 7.42(\operatorname{td}, J=4.5,4.1,2.0 \mathrm{~Hz}$, $3 \mathrm{H}), 7.36-7.20(\mathrm{~m}, 3 \mathrm{H}), 7.19-7.08(\mathrm{~m}, 2 \mathrm{H}), 6.02(\mathrm{~s}, 0.43 \mathrm{H}), 6.01(\mathrm{~s}, 0.48 \mathrm{H}), 3.77(\mathrm{~s}, 1.50 \mathrm{H})$, $3.76(\mathrm{~s}, 1.57 \mathrm{H}), 2.82-2.46(\mathrm{~m}, 1 \mathrm{H}), 2.20-2.05(\mathrm{~m}, 1 \mathrm{H}), 1.89-1.66(\mathrm{~m}, 1 \mathrm{H}), 1.56-1.29(\mathrm{~m}$, 1H) $\mathrm{ppm}$

${ }^{13}$ C NMR (101 MHz, Chloroform- $d$ ): $\delta=172.69,172.66,169.3,169.2,139.68,139.65,133.88$, $133.81,129.2,128.8,128.52,128,51,127.66,127.65 .126 .66,126.65,126.3,126.2,74.6,74.5$, 52.66, 52.64, 26.8, 26.7, 23.9, 23.8, 17.5, $17.4 \mathrm{ppm}$.

HRMS (ESI): $m / z$ : $[\mathrm{M}+\mathrm{Na}]^{+}$Calcd. for $\mathrm{C}_{19} \mathrm{H}_{18} \mathrm{NaO}_{4}{ }^{+}: 333.1097$; Found: 333.1093.

IR (KBr): 3451, 3033, 2954, 2328, 2154, 1730, 1604, 1497, 1438, 1404, 1345, 1257, 1216, $1157,1079,1046,1024,977,931,841,780,751,696 \mathrm{~cm}^{-1}$.

\section{2-Methoxy-2-oxo-1-phenylethyl 5-(1,2-dithiolan-3-yl)pentanoate (12k)}<smiles>COC(=O)C(OC(=O)CCCCC1CCCS1)c1ccccc1</smiles>

Compound 12k was prepared according to the general procedure GP-1 on a $0.2 \mathrm{mmol}$ scale and was obtained after silica column chromatography using $n$-hexane : EtOAc (40:1 $\rightarrow$ 9:1); 25\%, (12.5 mg); colorless solid.

${ }^{1}$ H NMR $(400 \mathrm{MHz}$, Chloroform- $d): \delta=7.59-7.44(\mathrm{~m}, 2 \mathrm{H}), 7.46-7.27(\mathrm{~m}, 3 \mathrm{H}), 5.96(\mathrm{~s}, 1 \mathrm{H})$, $3.75(\mathrm{~s}, 3 \mathrm{H}), 3.66-3.49(\mathrm{~m}, 1 \mathrm{H}), 3.30-2.83(\mathrm{~m}, 2 \mathrm{H}), 2.57-2.35(\mathrm{~m}, 3 \mathrm{H}), 2.04-1.86(\mathrm{~m}$, $1 \mathrm{H}), 1.82-1.69(\mathrm{~m}, 4 \mathrm{H}), 1.58-1.46(\mathrm{~m}, 2 \mathrm{H}) \mathrm{ppm}$.

${ }^{13}$ C NMR (101 MHz, Chloroform- $d$ ): $\delta=172.8,169.3,133.8,129.2,128.8,127.6,74.3,56.2$, 52.6, 40.1, 38.4, 34.5, 33.7, 28.62, 28.60, 24.56, $24.54 \mathrm{ppm}$.

HRMS (ESI): $m / z$ : $[\mathrm{M}+\mathrm{Na}]^{+}$Calcd. for $\mathrm{C}_{17} \mathrm{H}_{22} \mathrm{NaO}_{4} \mathrm{~S}_{2}^{+}: 377.0851$; Found: 377.0848 .

IR (KBr): 3482, 3034, 2931, 2857, 2672, 2326, 2092, 1741, 1588, 1496, 1436, 1352, 1268 , $1215,1158,1082,1040,978,940,852,735,696 \mathrm{~cm}^{-1}$. 


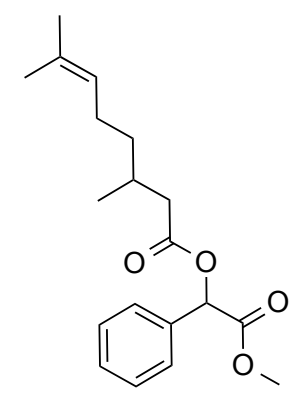

Compound 121 was prepared according to the general procedure GP- 1 on a $0.2 \mathrm{mmol}$ scale and was obtained after silica column chromatography using $n$-hexane : EtOAc $(40: 1 \rightarrow 9: 1) ; 85 \%$, (54.4 mg); d.r. 1: 1.1; colorless oil.

${ }^{1}$ H NMR (400 MHz, Chloroform- $d$ ): $\delta=7.50-7.42(\mathrm{~m}, 2 \mathrm{H}), 7.41-7.33(\mathrm{~m}, 3 \mathrm{H}), 5.91$ (s, $0.40 \mathrm{H}), 5.91(\mathrm{~s}, 0.44 \mathrm{H}), 5.19-5.00(\mathrm{~m}, 1 \mathrm{H}), 3.70(\mathrm{~s}, 3 \mathrm{H}), 2.56-2.38(\mathrm{~m}, 1 \mathrm{H}), 2.34-2.16(\mathrm{~m}$, $1 \mathrm{H}), 2.11-1.89(\mathrm{~m}, 3 \mathrm{H}), 1.65(\mathrm{~s}, 3 \mathrm{H}), 1.57(\mathrm{~s}, 3 \mathrm{H}), 1.45-1.30(\mathrm{~m}, 1 \mathrm{H}), 1.29-1.19(\mathrm{~m}, 1 \mathrm{H})$, $0.99(\mathrm{~s}, 3 \mathrm{H}) \mathrm{ppm}$.

${ }^{13}$ C NMR (101 MHz, Chloroform- $\left.d\right): \delta=172.57,172.51,169.3,133.8,131.5,129.1,128.7$, $127.5,124.2,74.28,74.22,52.5,41.34,41.30,36.67,36.63,30.0,29.9,25.6,25.3,19.5,19.4$, $17.6 \mathrm{ppm}$.

HRMS (ESI): $m / z$ : $[\mathrm{M}+\mathrm{Na}]^{+}$Calcd. for $\mathrm{C}_{19} \mathrm{H}_{26} \mathrm{NaO}_{4}{ }^{+}: 341.1723$; Found: 341.1721.

IR (KBr): 3483, 3034, 2959, 2919, 2729, 2323, 2163, 2087, 1902, 1743, 1605, 1496, 1440, $1374,1351,1260,1214,1145,1083,1043,983,947,828,782,734,695 \mathrm{~cm}^{-1}$. 


\section{References}

[1] Ochoa, G.; Pilgrim, C. D.; Kerr, J.; Augustine, M. P.; Casey, W. H. Aqueous geochemistry at gigapascal pressures: NMR spectroscopy of fluoro- borate solutions, Geochimica et Cosmochimica Acta, 2019, 244, 173-181.

[2] Keipour, H.; Ollevier, T. Iron-Catalyzed Carbene Insertion Reactions of $\alpha$-Diazoesters into Si-H Bonds, Org. Lett. 2017, 19, 5736-5739.

[3] Thurow, S.; Fernandes, A. A. G.; Quevedo-Acosta, Y.; Oliveira de, M. F.; Oliveria de, M. G. Jurberg, I. D. Preparation of Organic Nitrates from Aryldiazoacetates and $\mathrm{Fe}\left(\mathrm{NO}_{3}\right)_{3} \cdot 9 \mathrm{H}_{2} \mathrm{O}$, Org. Lett. 2019, 17, 6909-6913.

[4] Green, P. S.; Wheelhouse, K. M.; Payne, A. D.; Hallett, J. P.; Miller, W. P.; Bull, J. A. Thermal Stability and Explosive Hazard Assessment of Diazo Compounds and Diazo Transfer Reagents, Org. Process Res. Dev. 2020, 24, 67-84.

[5] Boody, A. J.; Affron, D. P.; Cordier, C. J.; Rivers, E. L.; Spivey, A. C.; Bull, J. A. Rapid Assembly of Saturated Nitrogen Heterocycles in One-Pot: Diazo-Heterocycle "Stitching" by $\mathrm{N}-\mathrm{H}$ Insertion and Cyclization, Angew. Chem. Int. Ed. 2019, 58, 1458-1462. 


\section{Spectra}

\section{2-Methoxy-2-oxo-1-phenylethyl benzoate (7a)}

${ }^{1}$ H NMR (600 MHz, Chloroform- $d$ )

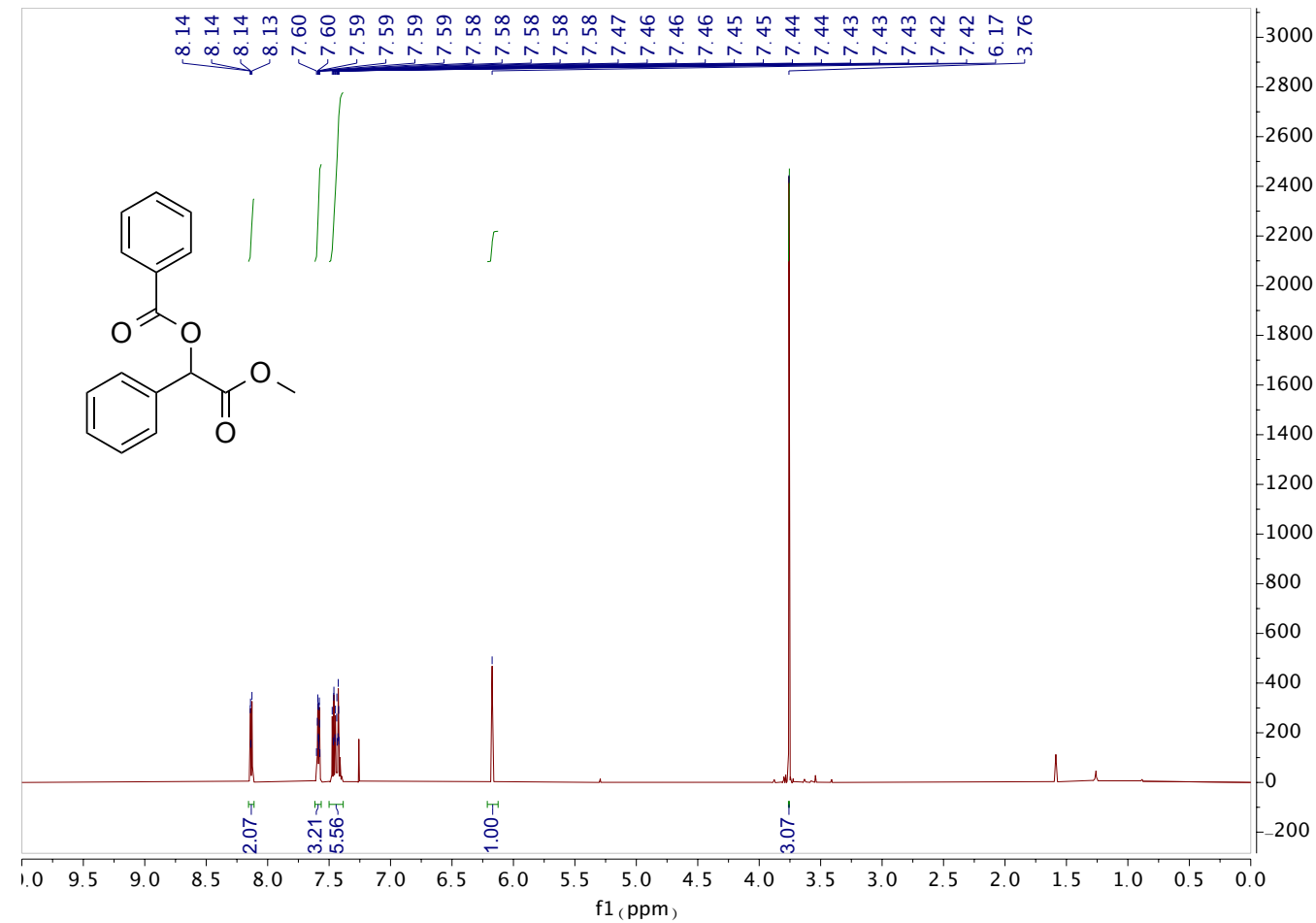

${ }^{13}$ C NMR (151 MHz, Chloroform- $d$ )

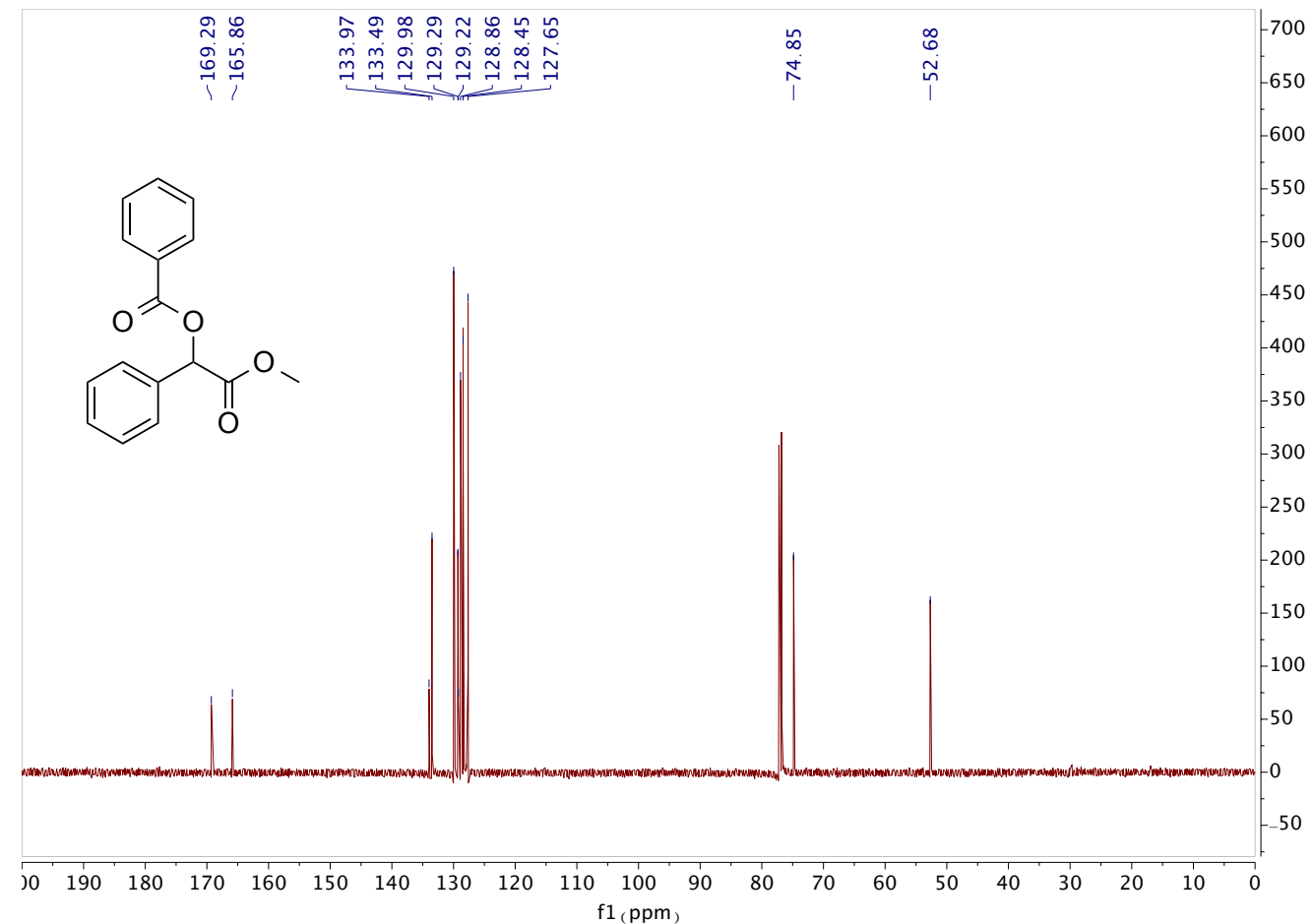


2-Ethoxy-2-oxo-1-phenylethyl benzoate (7b)

${ }^{1}$ H NMR (400 MHz, Chloroform- $d$ )

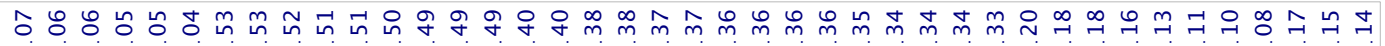

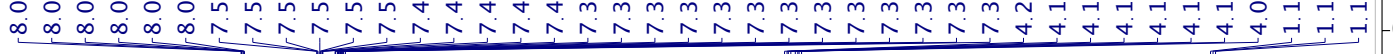

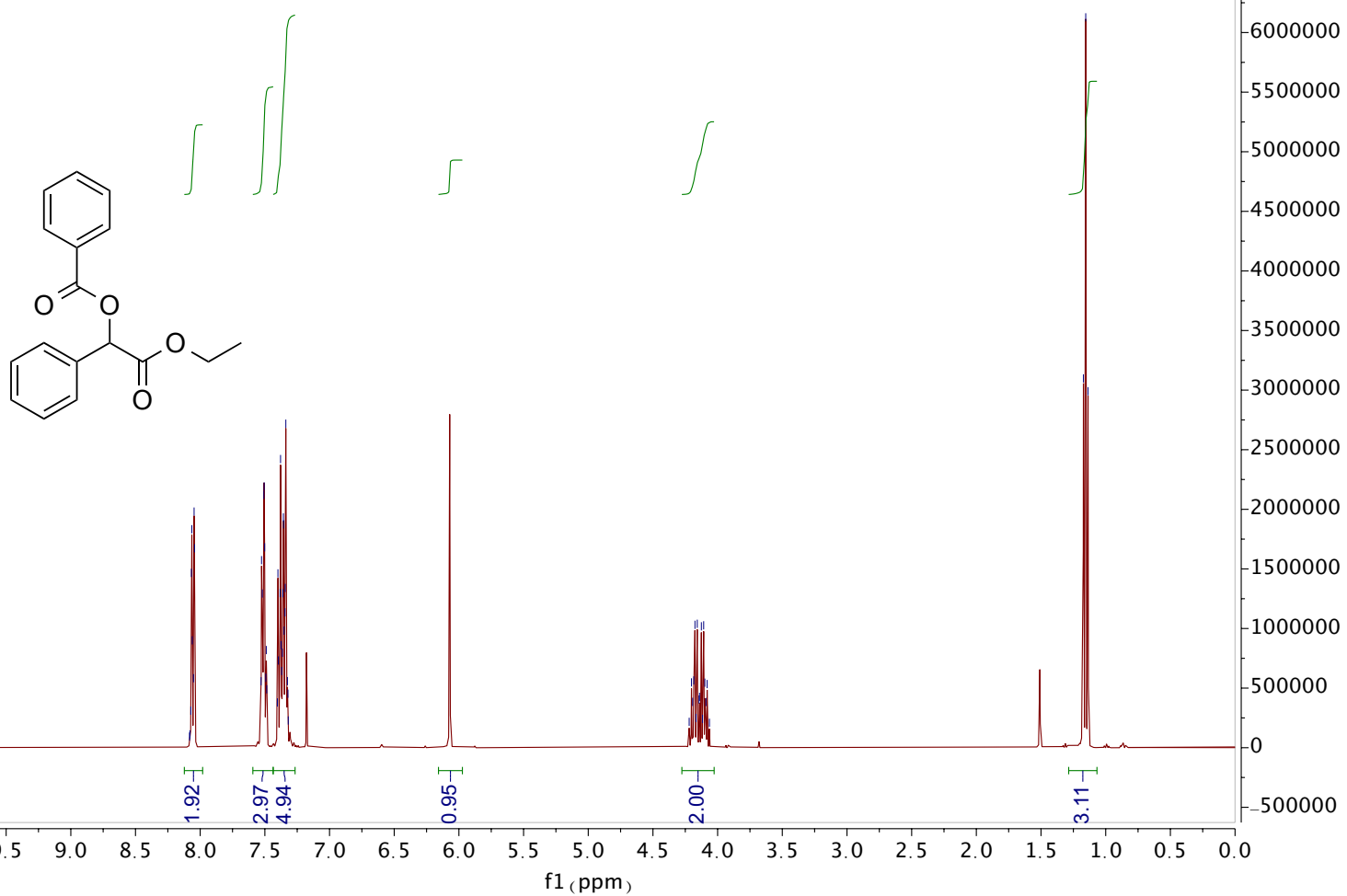

${ }^{13}$ C NMR (101 MHz, Chloroform- $d$ )

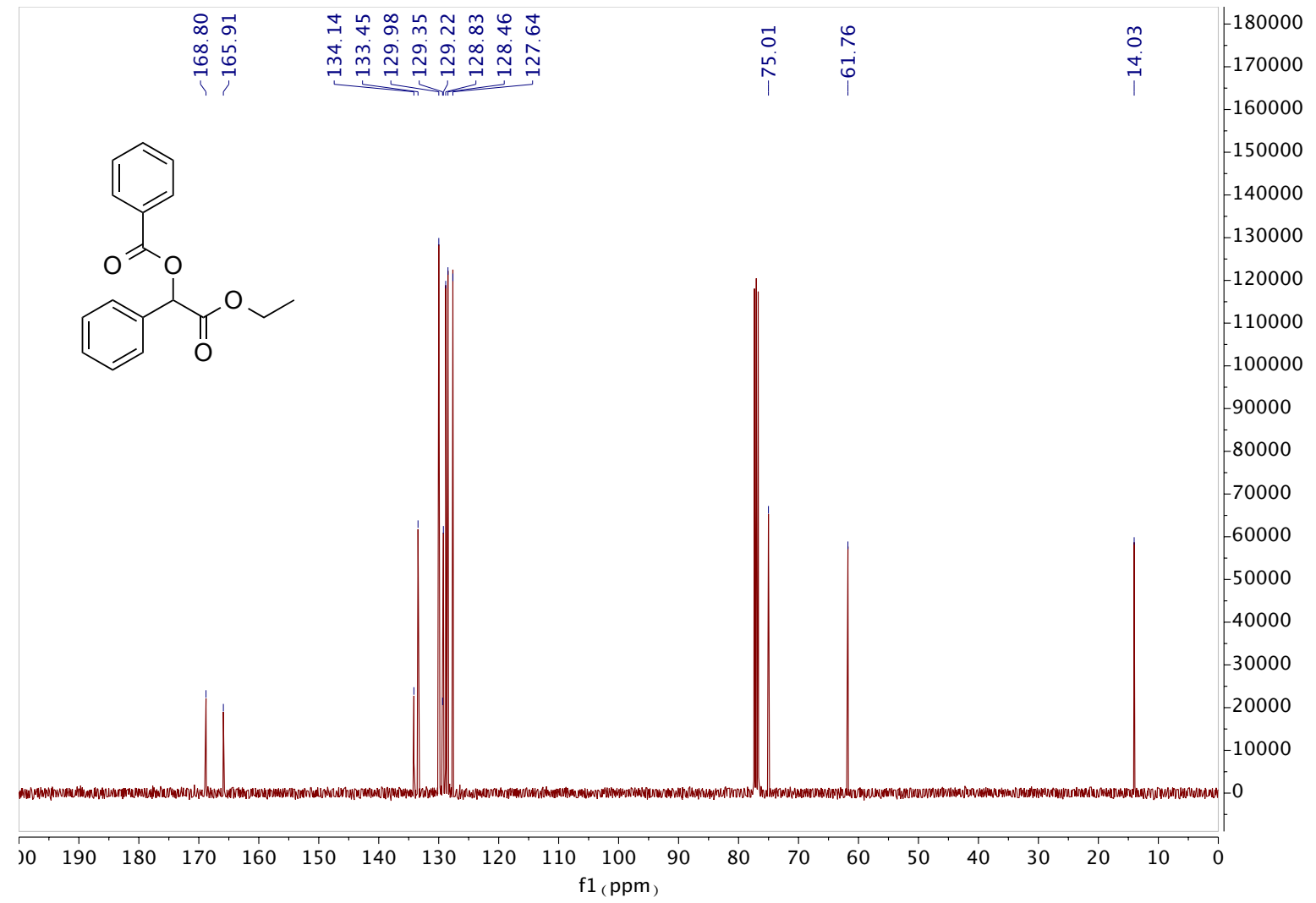




\section{2-(Cyclohexyloxy)-2-oxo-1-phenylethyl benzoate (7c)}

${ }^{1}$ H NMR (400 MHz, Chloroform- $d$ )

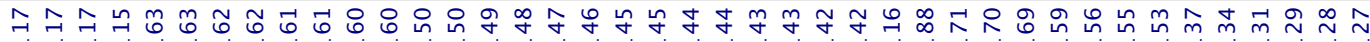

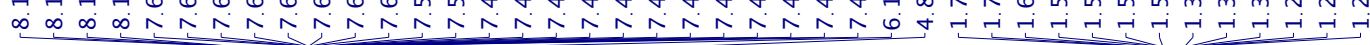

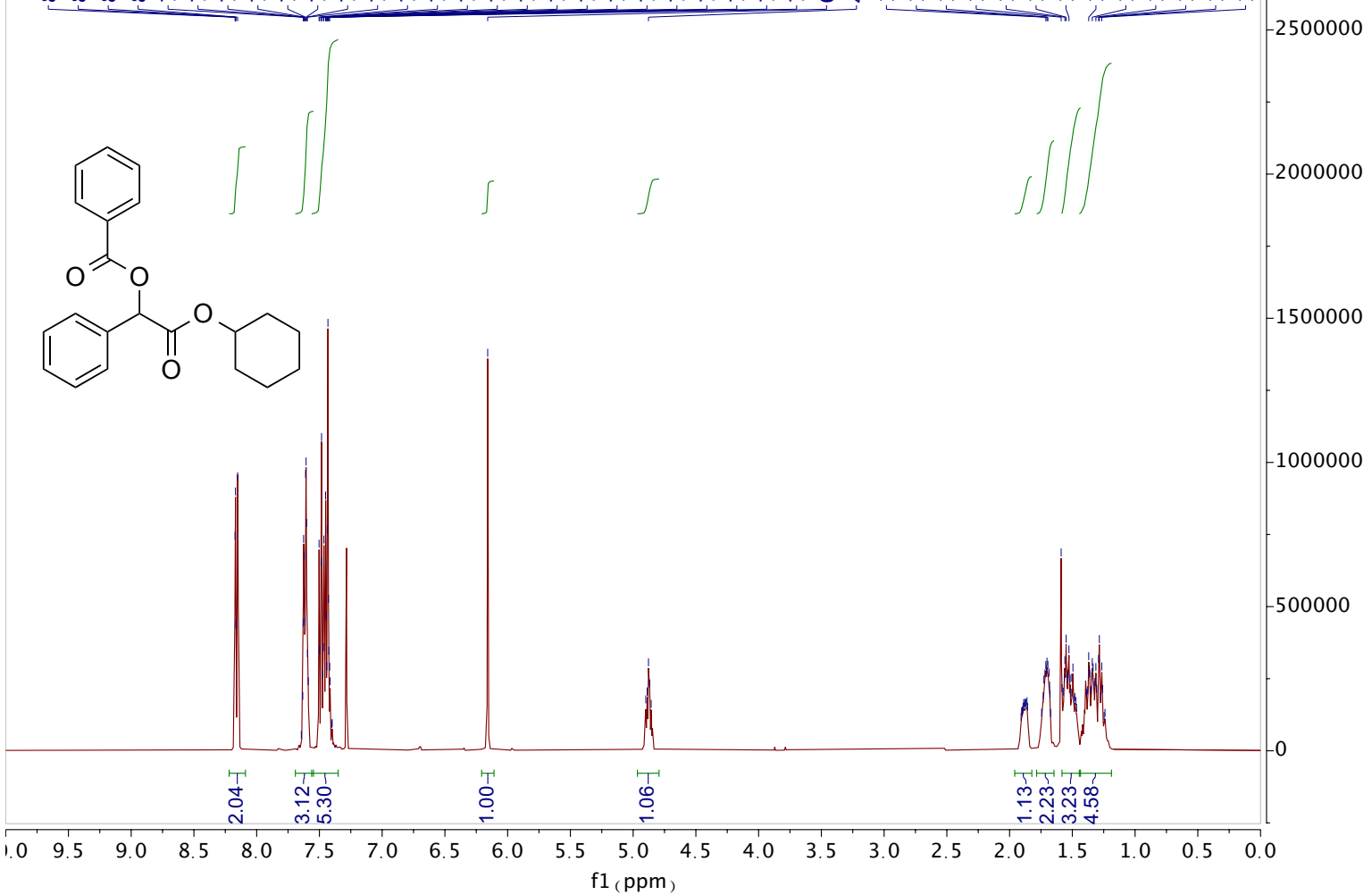

${ }^{13}$ C NMR (101 MHz, Chloroform- $d$ )

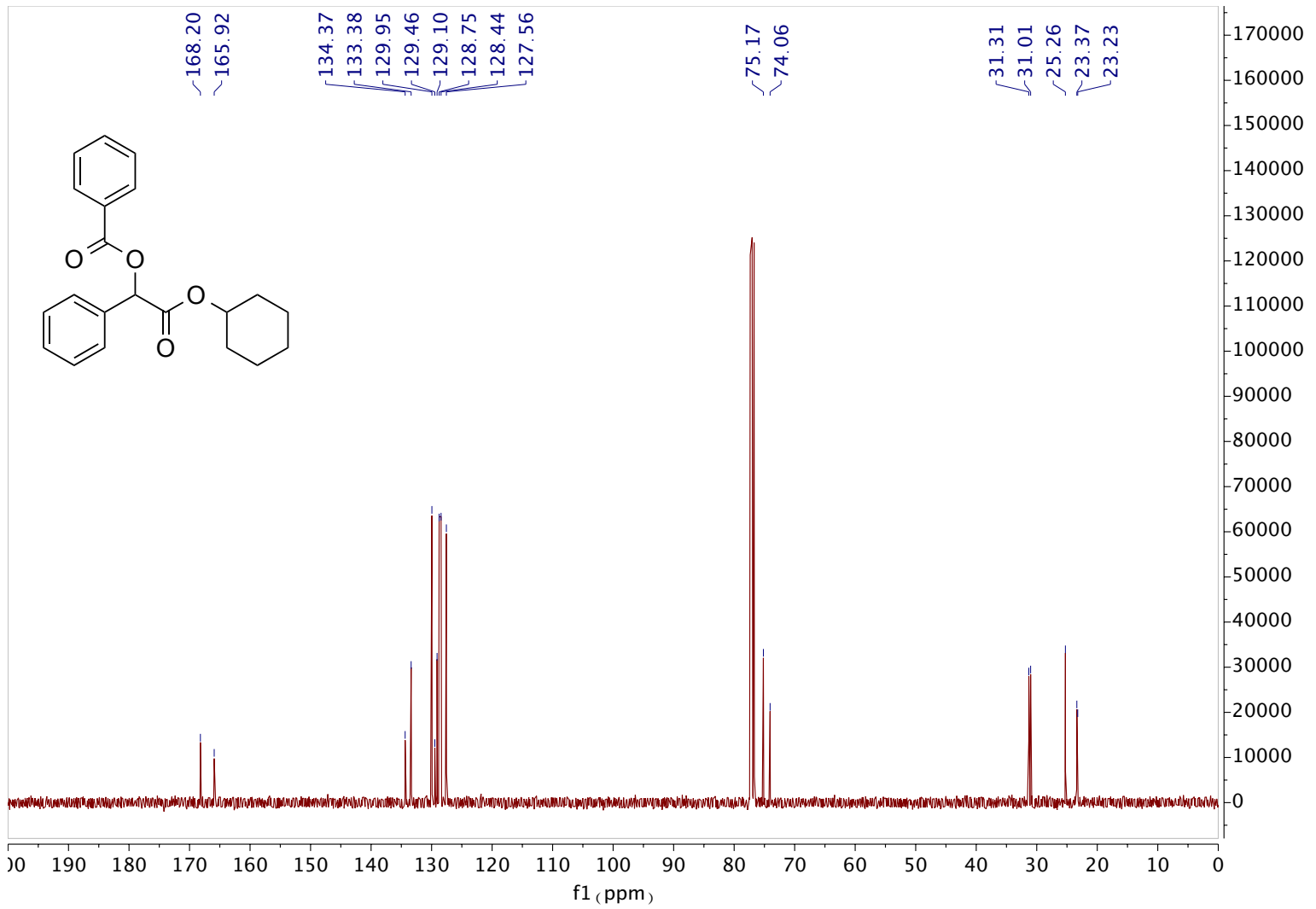


${ }^{1}$ H NMR (400 MHz, Chloroform- $d$ )

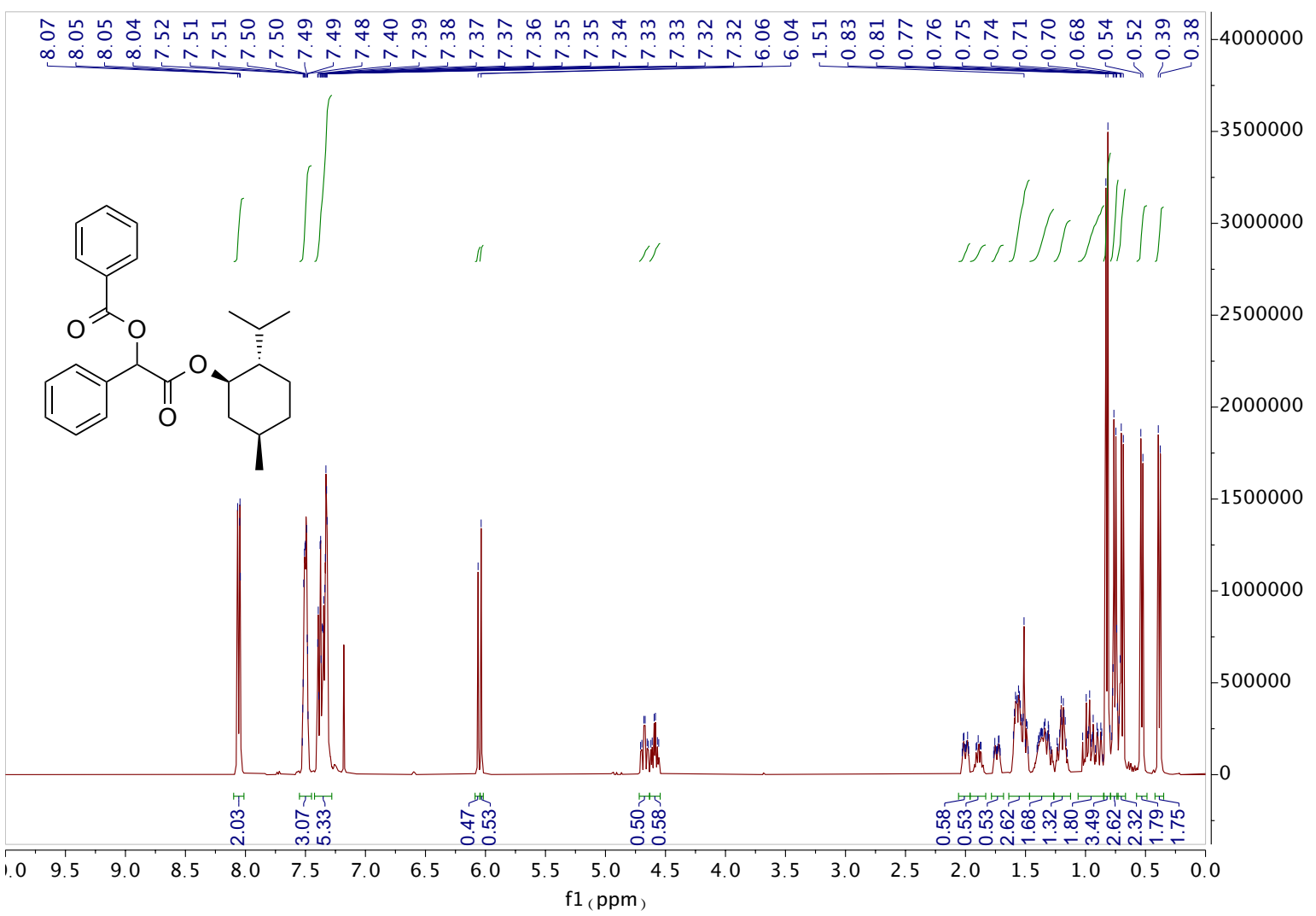

${ }^{13}$ C NMR (101 MHz, Chloroform- $d$ )

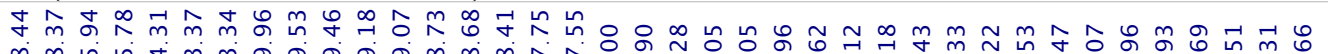

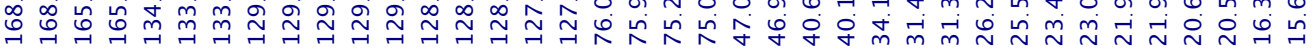

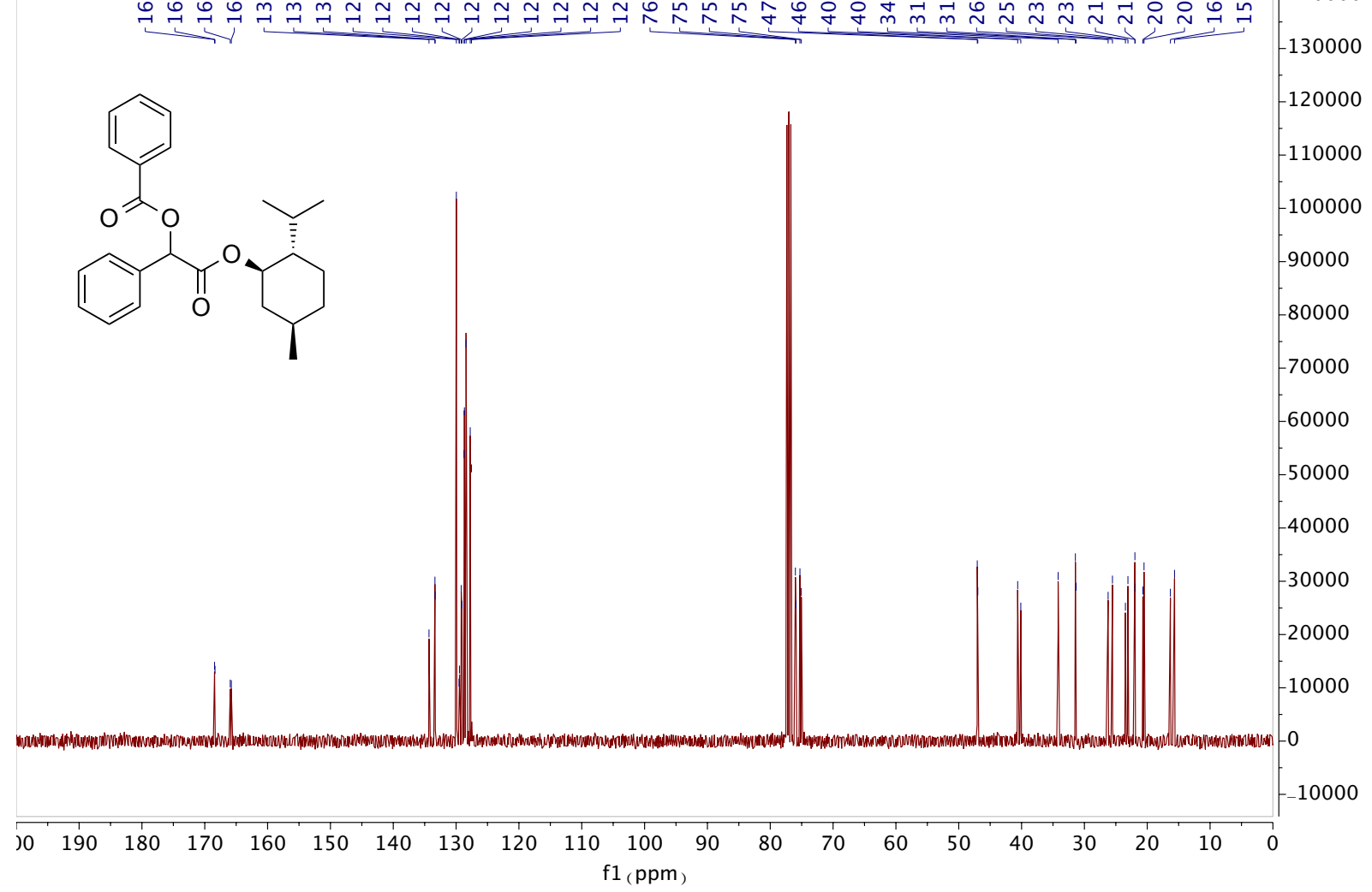




\section{2-(tert-Butoxy)-2-oxo-1-phenylethyl benzoate (7e)}

${ }^{1}$ H NMR (400 MHz, Chloroform- $d$ )

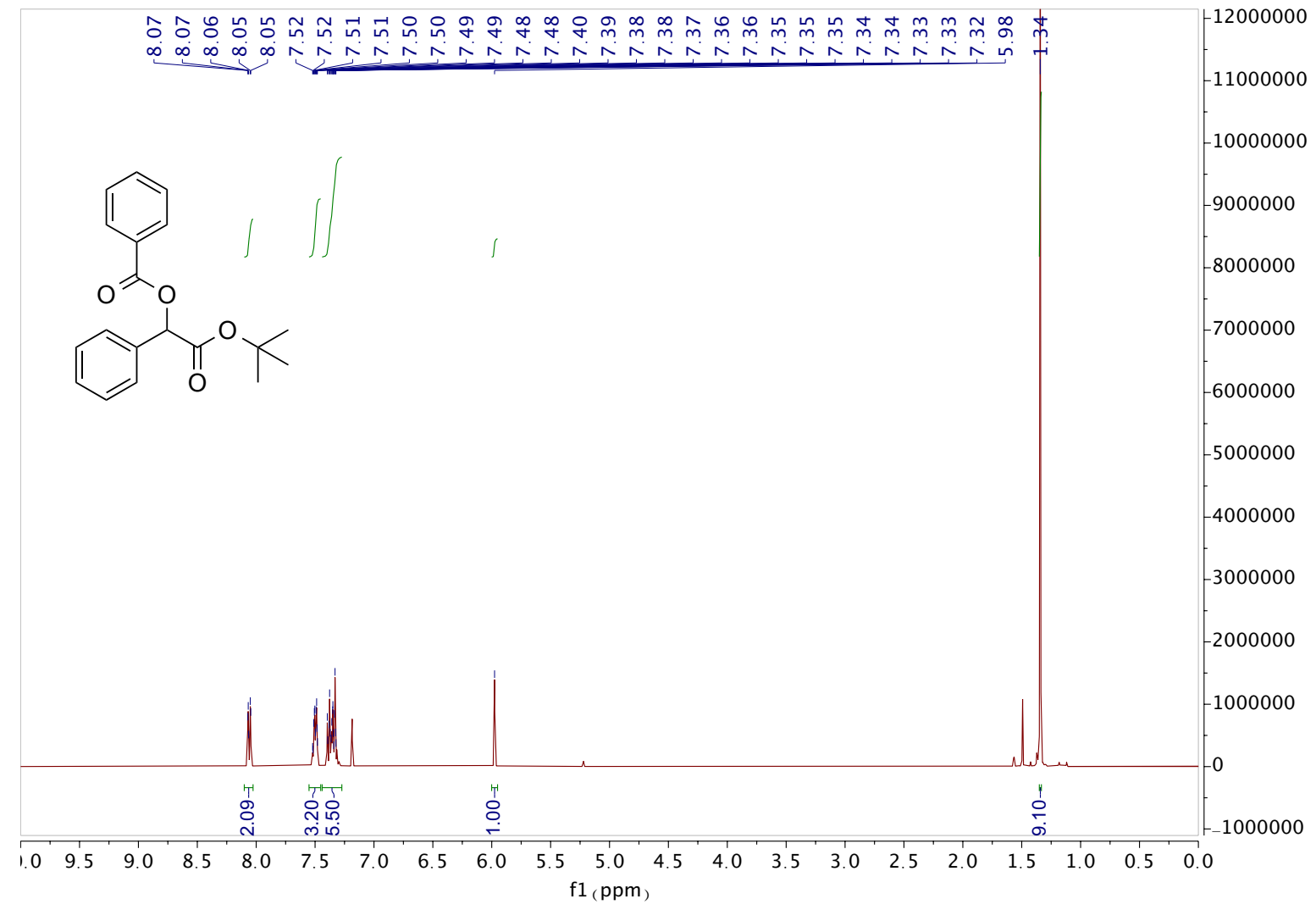

${ }^{13}$ C NMR (101 MHz, Chloroform- $d$ )

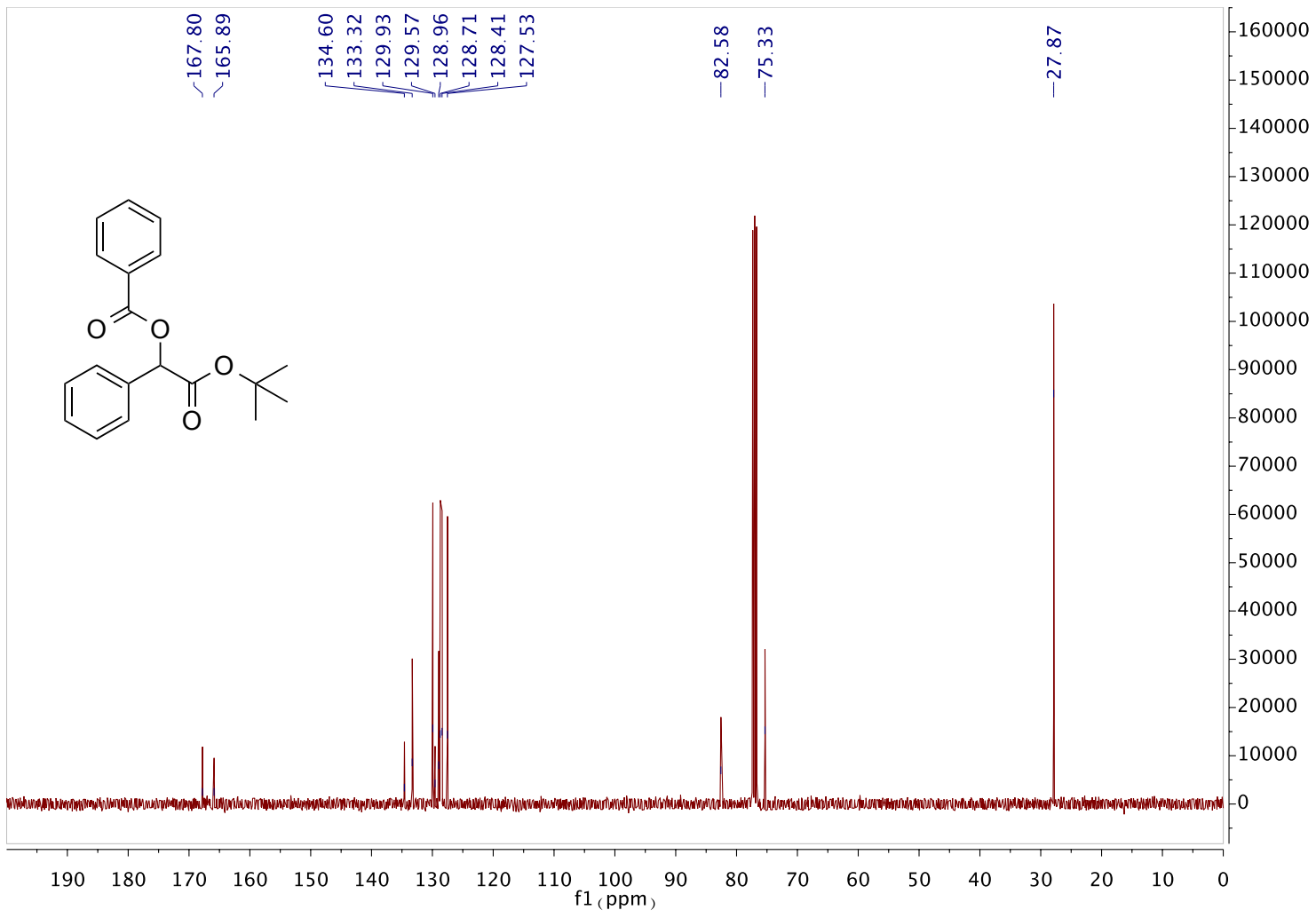


${ }^{1}$ H NMR (400 MHz, Chloroform- $d$ )

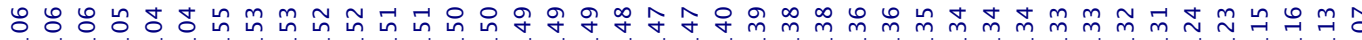

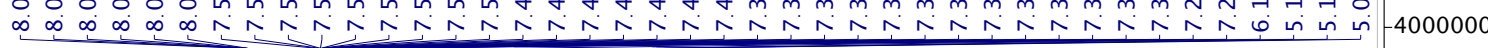
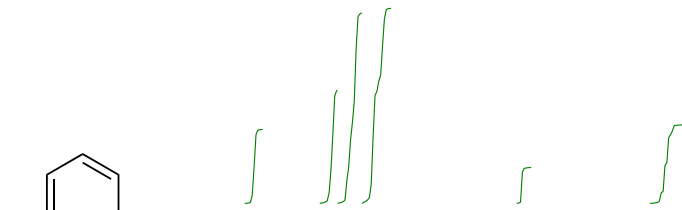

3500000

1

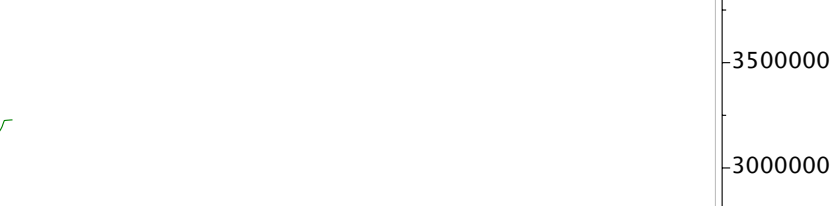<smiles>CC(C(=O)OCC1=CCC=C1)c1ccccc1</smiles><smiles>c1ccc(C23CCCCC2C3)cc1</smiles>

2500000

2000000

1500000

1000000

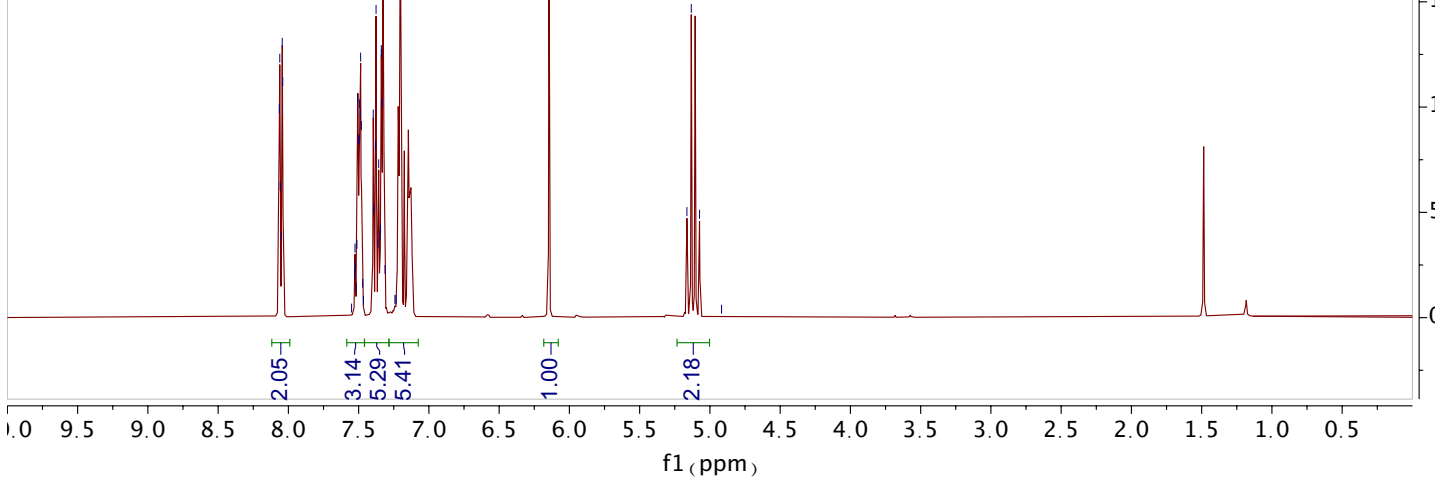

${ }^{13}$ C NMR (101 MHz, Chloroform- $d$ )

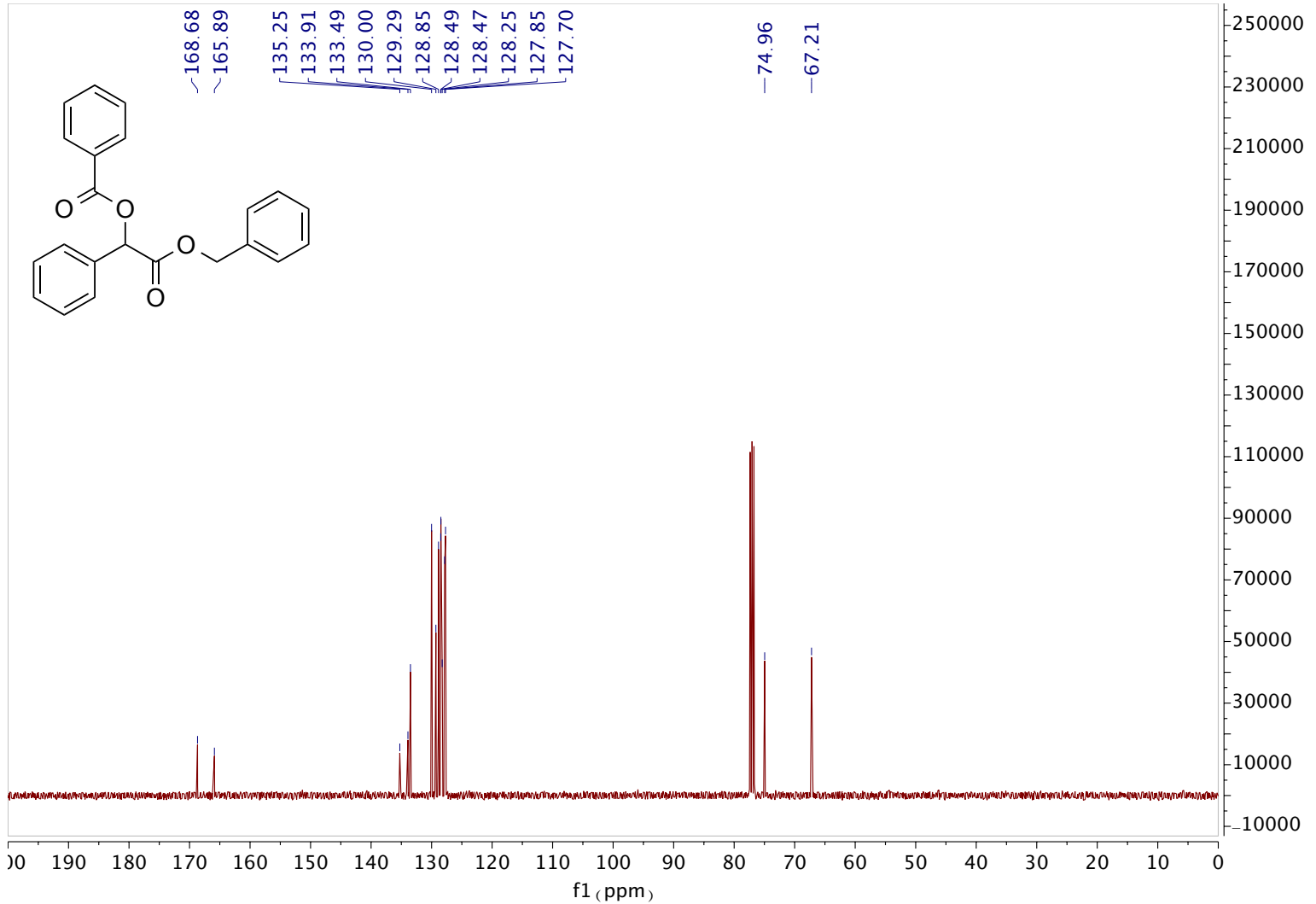




\subsection{1-(4-Fluorophenyl)-2-methoxy-2-oxoethyl benzoate (7g)}

${ }^{1}$ H NMR (600 MHz, Chloroform- $d$ )

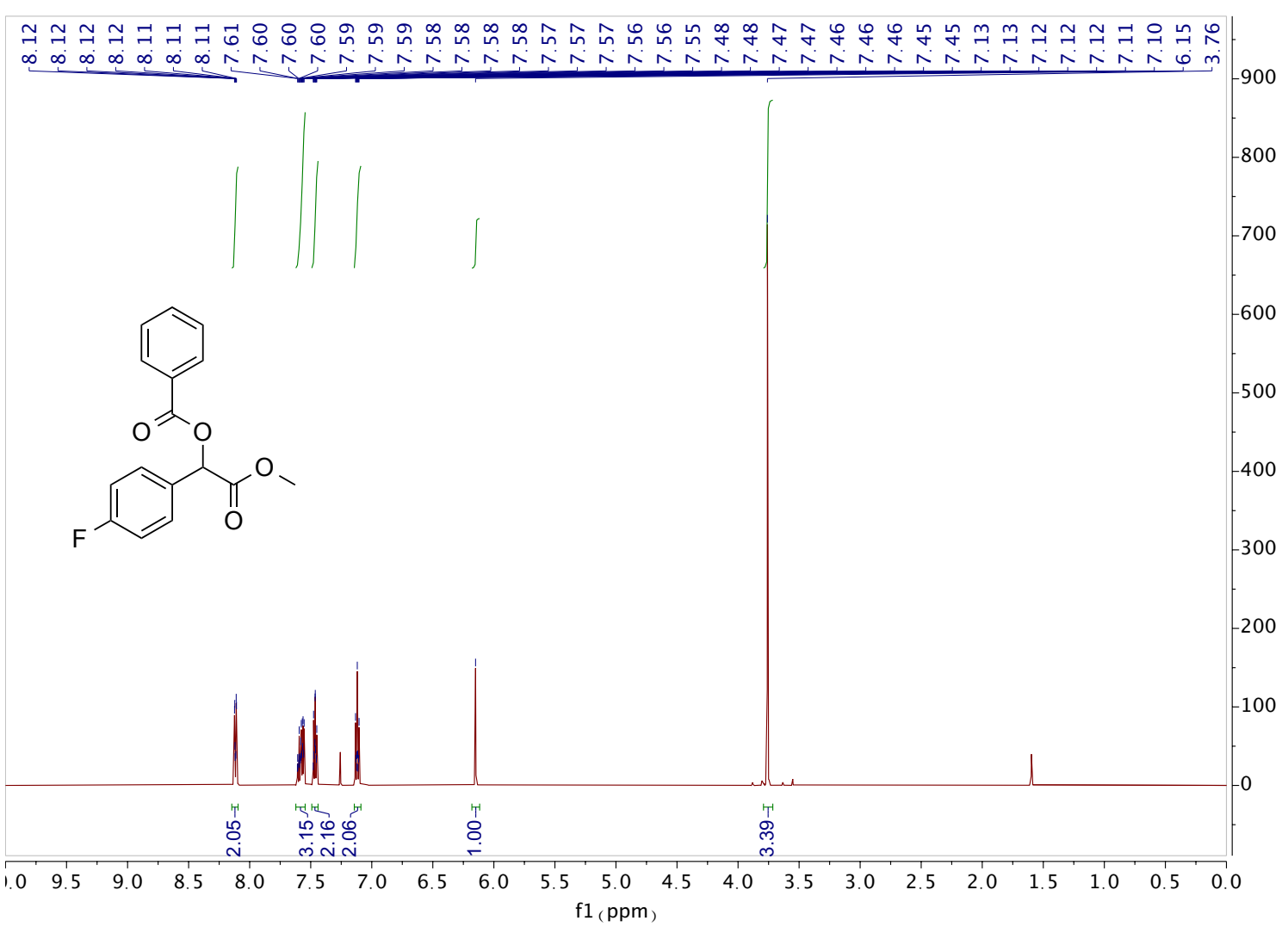

${ }^{13}$ C NMR (151 MHz, Chloroform- $d$ )

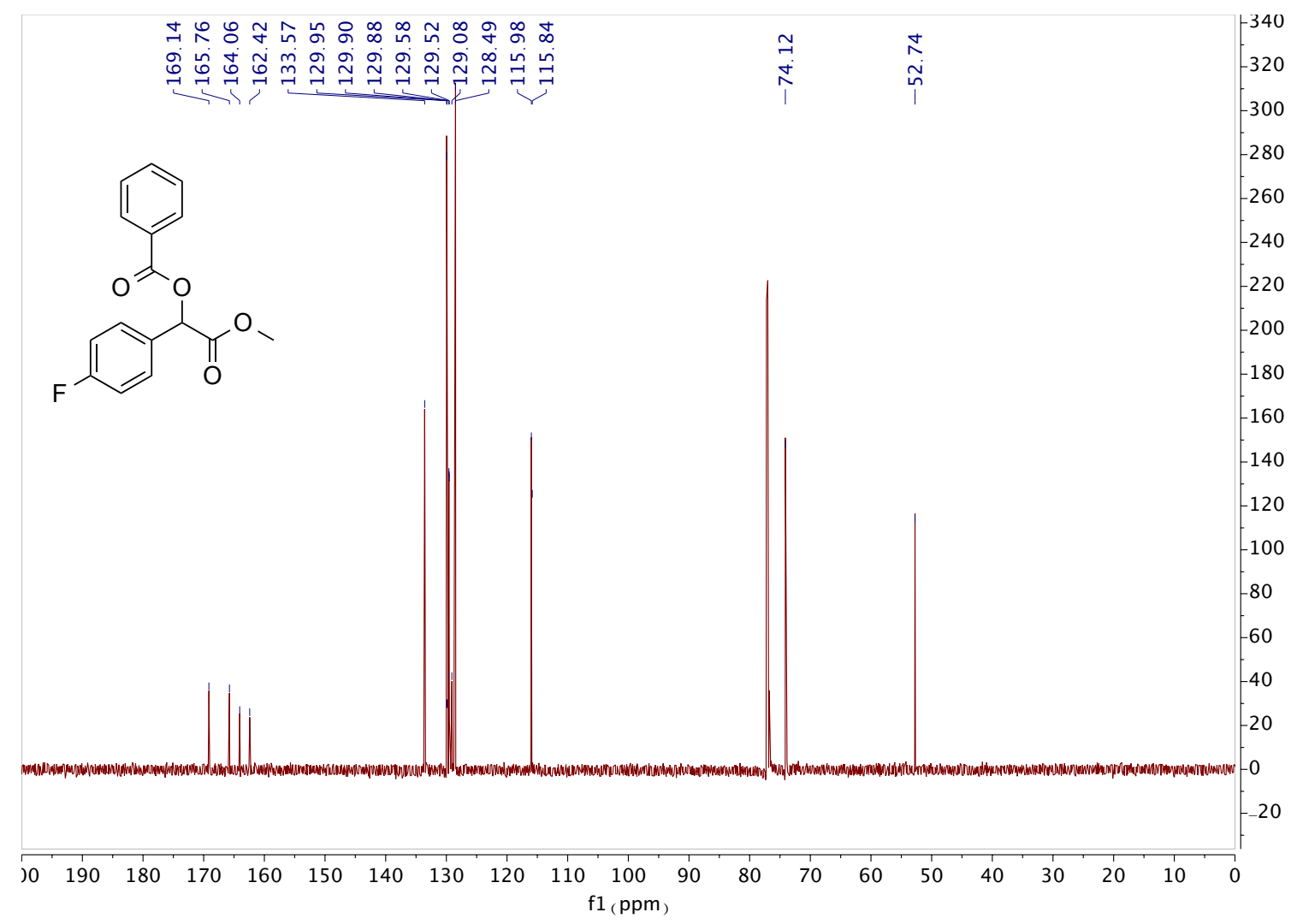


${ }^{19}$ F NMR (565 MHz, Chloroform- $d$ )

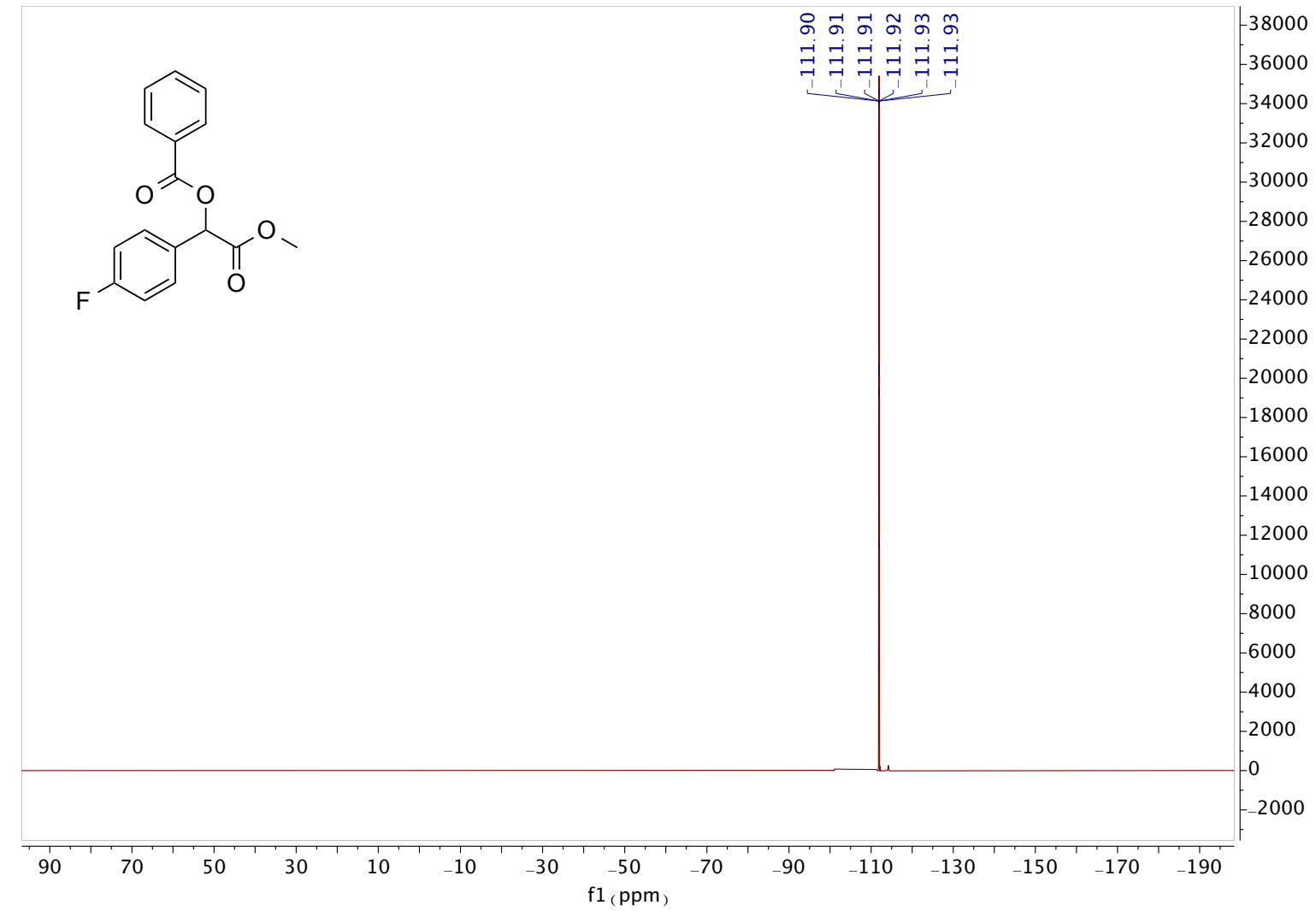




\section{1-(4-Chlorophenyl)-2-methoxy-2-oxoethyl benzoate (7h)}

${ }^{1}$ H NMR (400 MHz, Chloroform- $d$ )

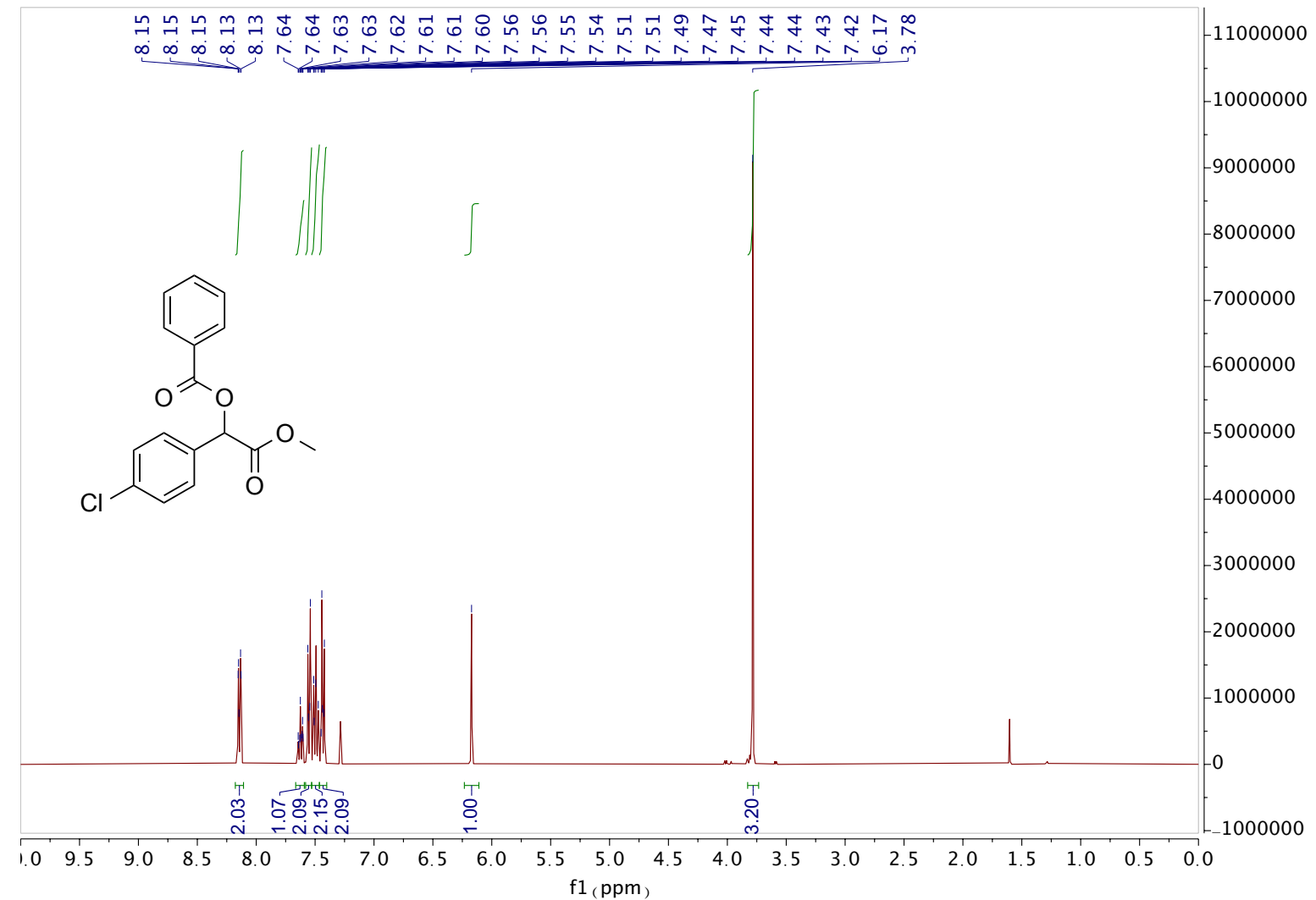

${ }^{13}$ C NMR (101 MHz, Chloroform- $d$ )

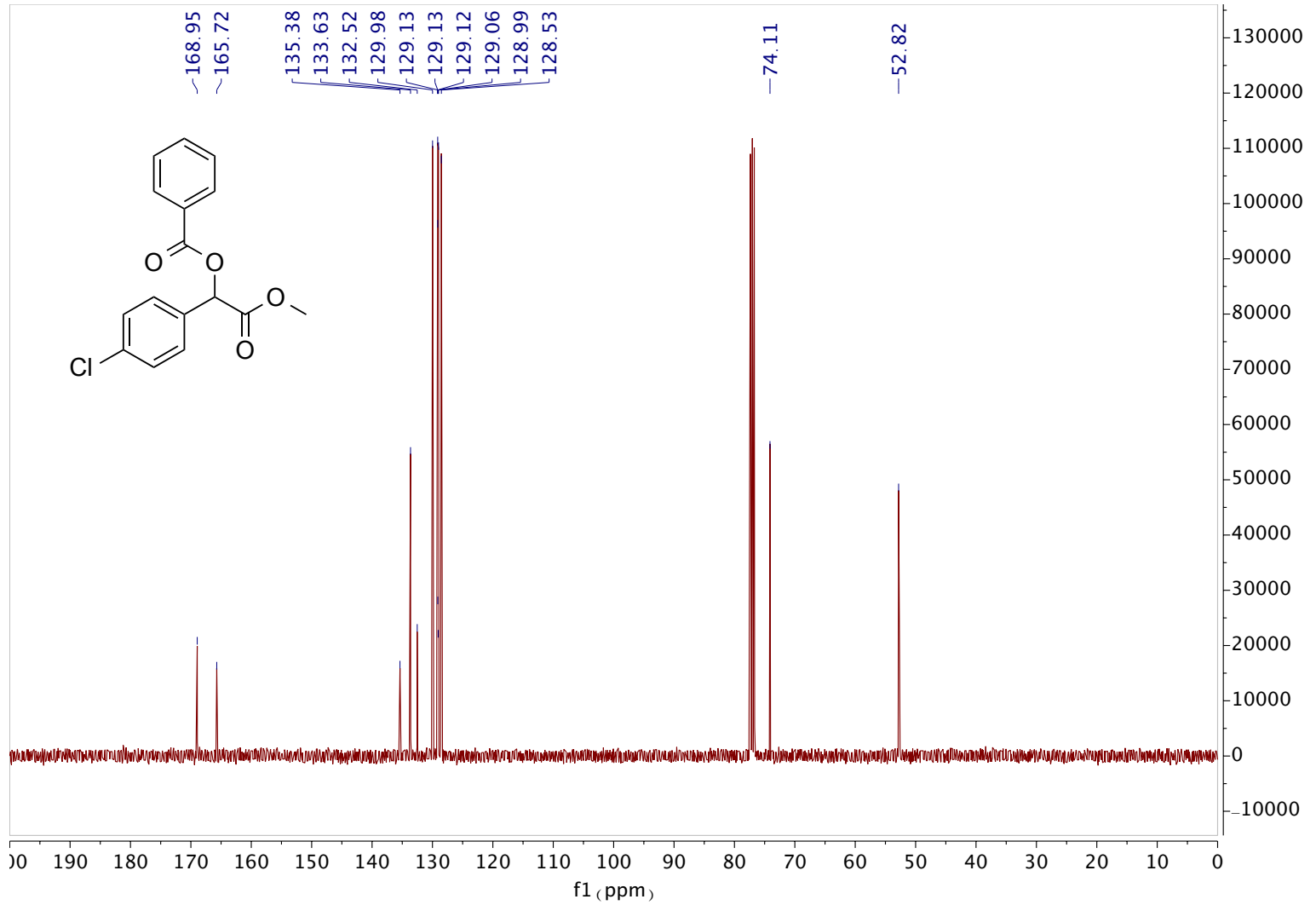




\section{1-(4-Bromophenyl)-2-methoxy-2-oxoethyl benzoate (7i)}

${ }^{1}$ H NMR (400 MHz, Chloroform- $d$ )

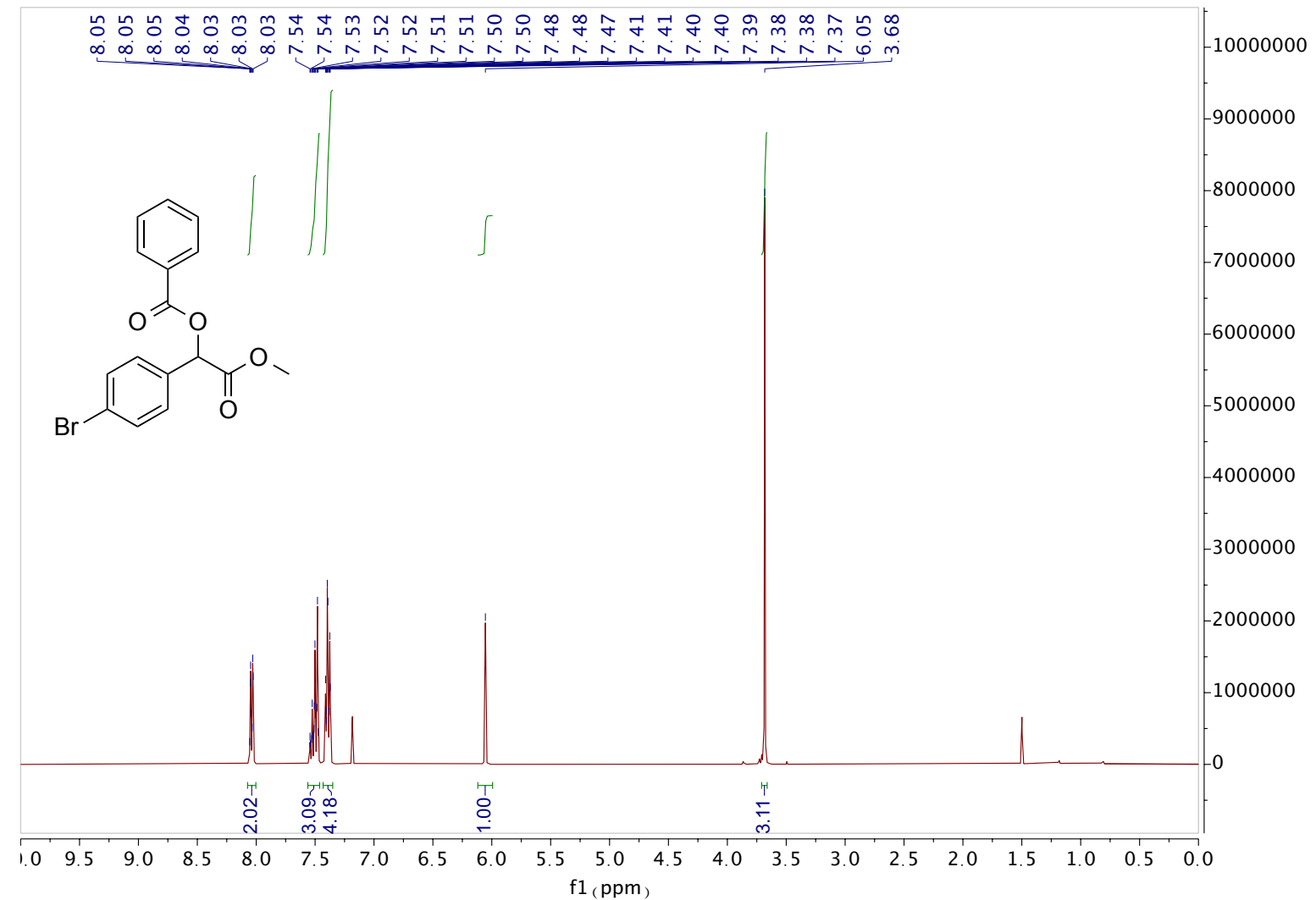

${ }^{13}$ C NMR (101 MHz, Chloroform- $d$ )

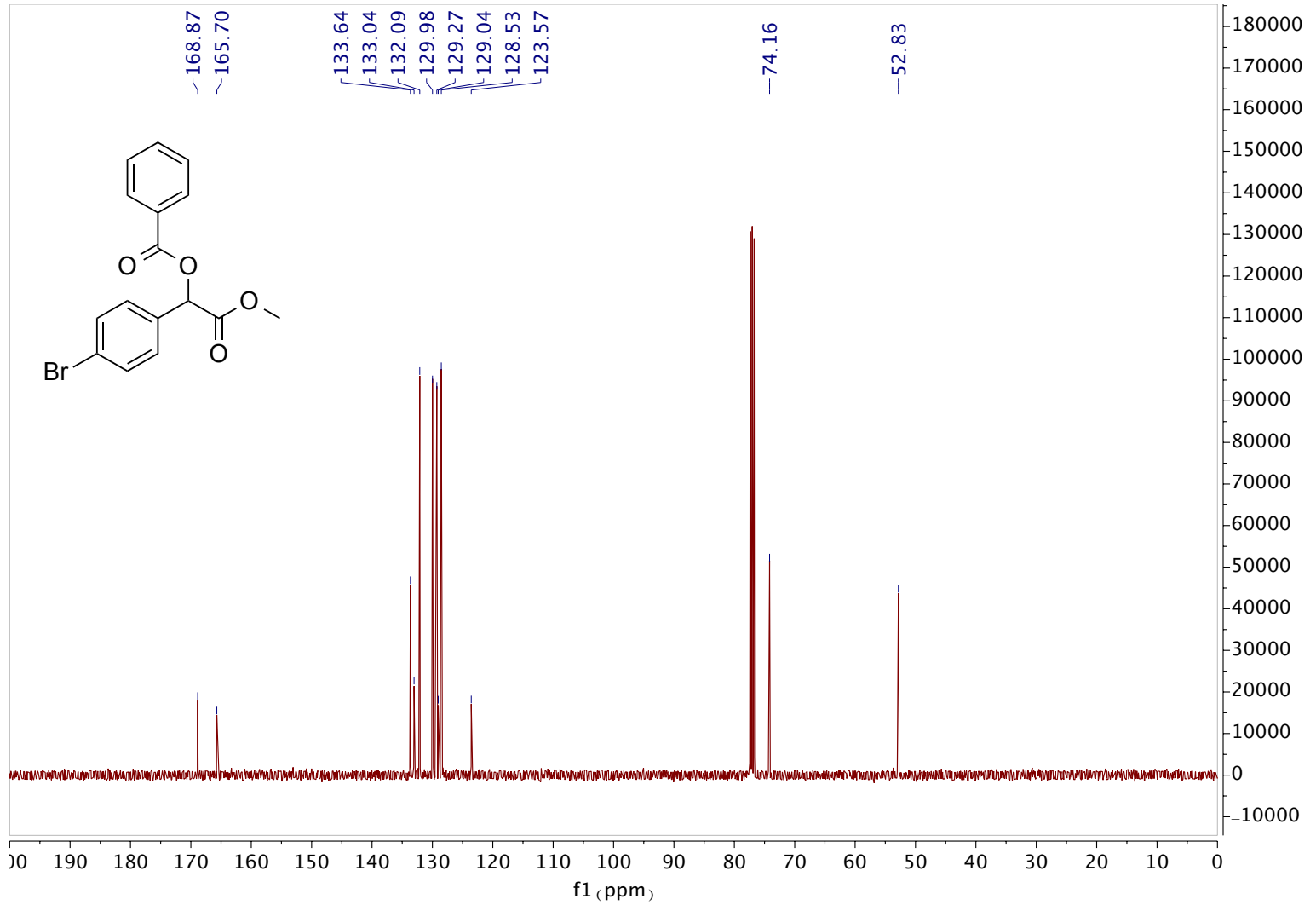


1-(4-Bromophenyl)-2-ethoxy-2-oxoethyl benzoate $(7 \mathbf{j})$

${ }^{1}$ H NMR (400 MHz, Chloroform- $d$ )

능 농

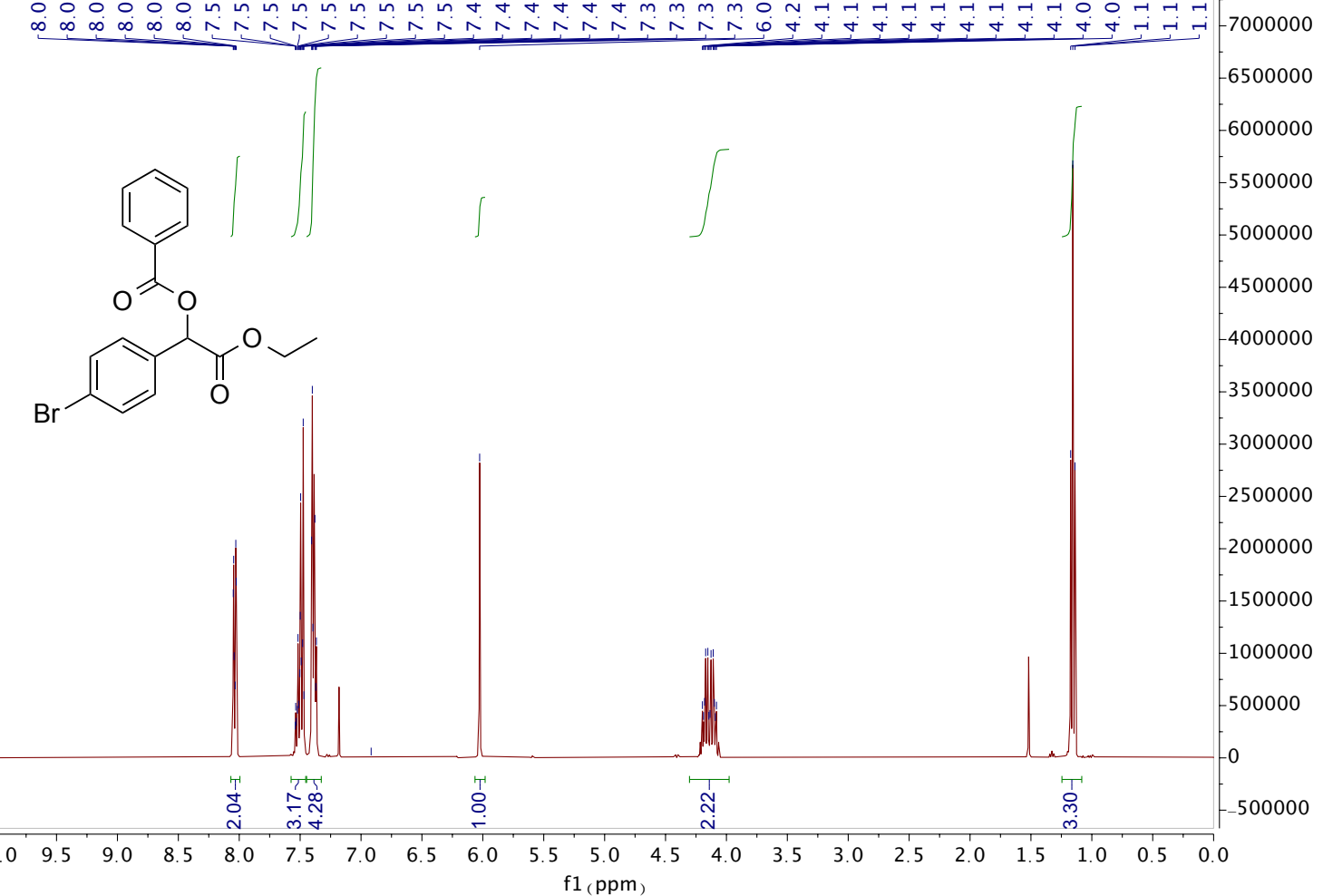

${ }^{13}$ C NMR (151 MHz, Chloroform- $d$ )

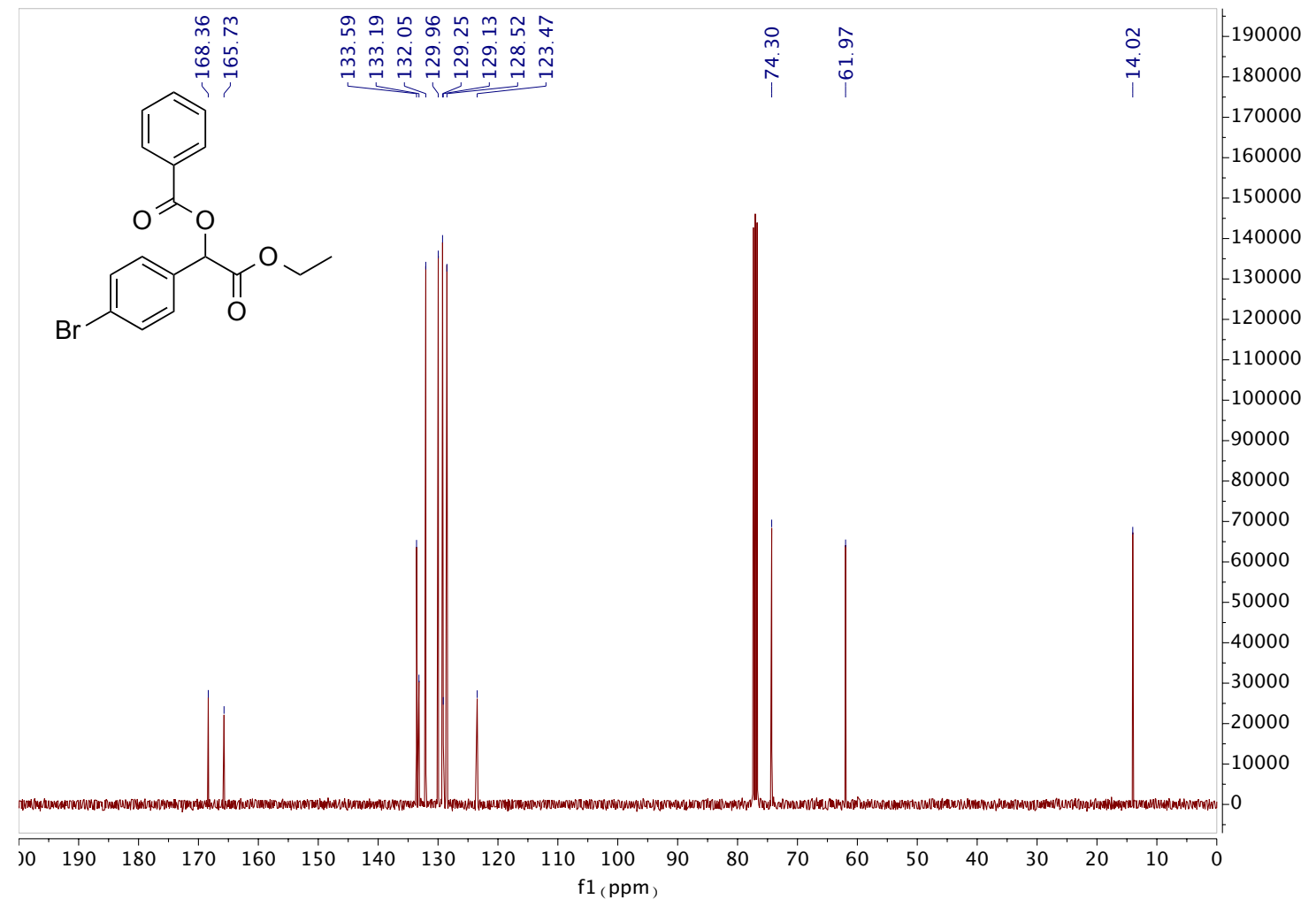


${ }^{1}$ H NMR (400 MHz, Chloroform- $d$ )

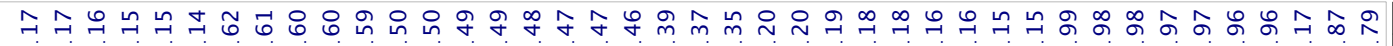

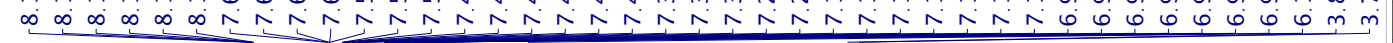

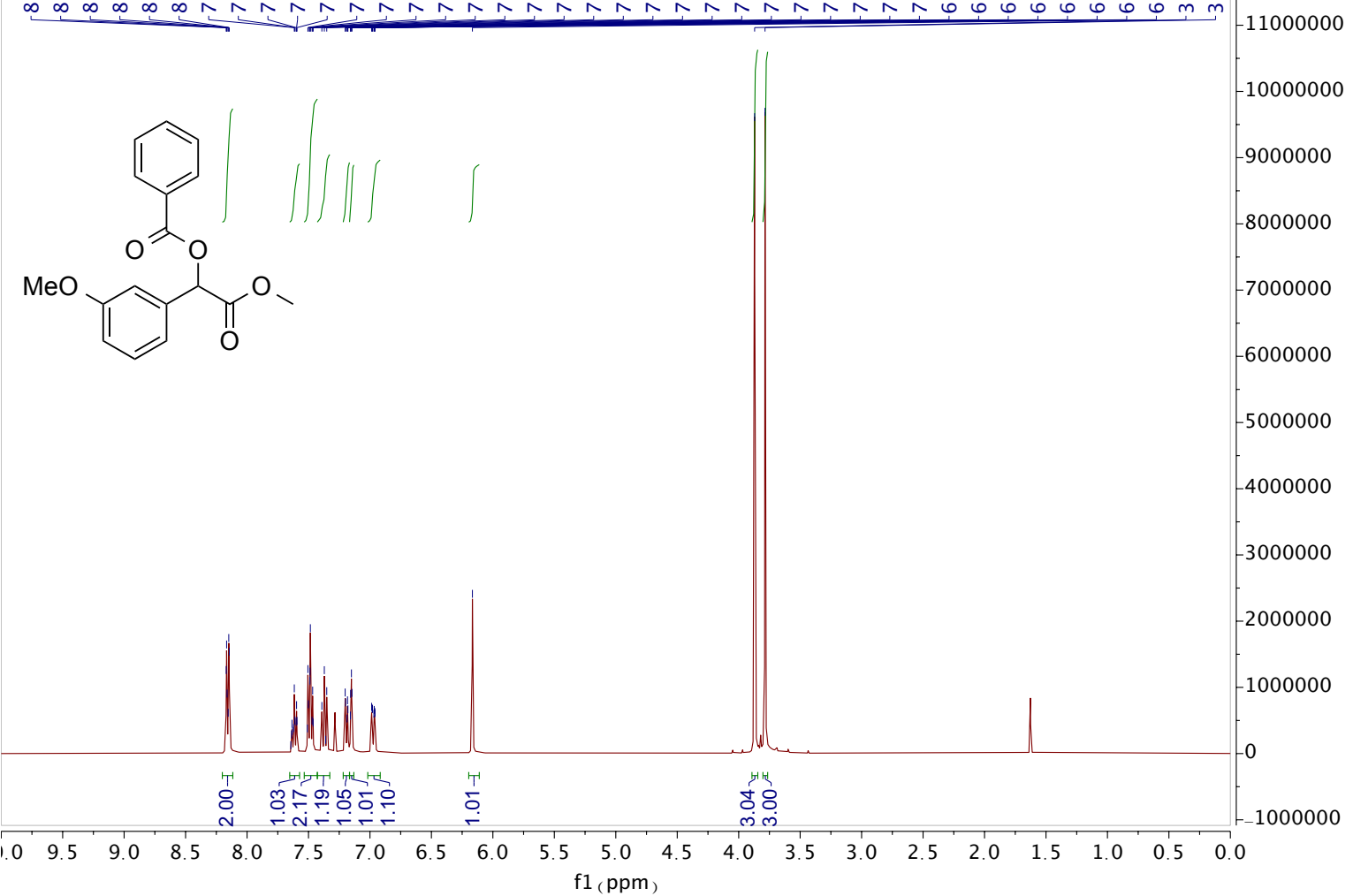

${ }^{13}$ C NMR (101 MHz, Chloroform- $d$ )

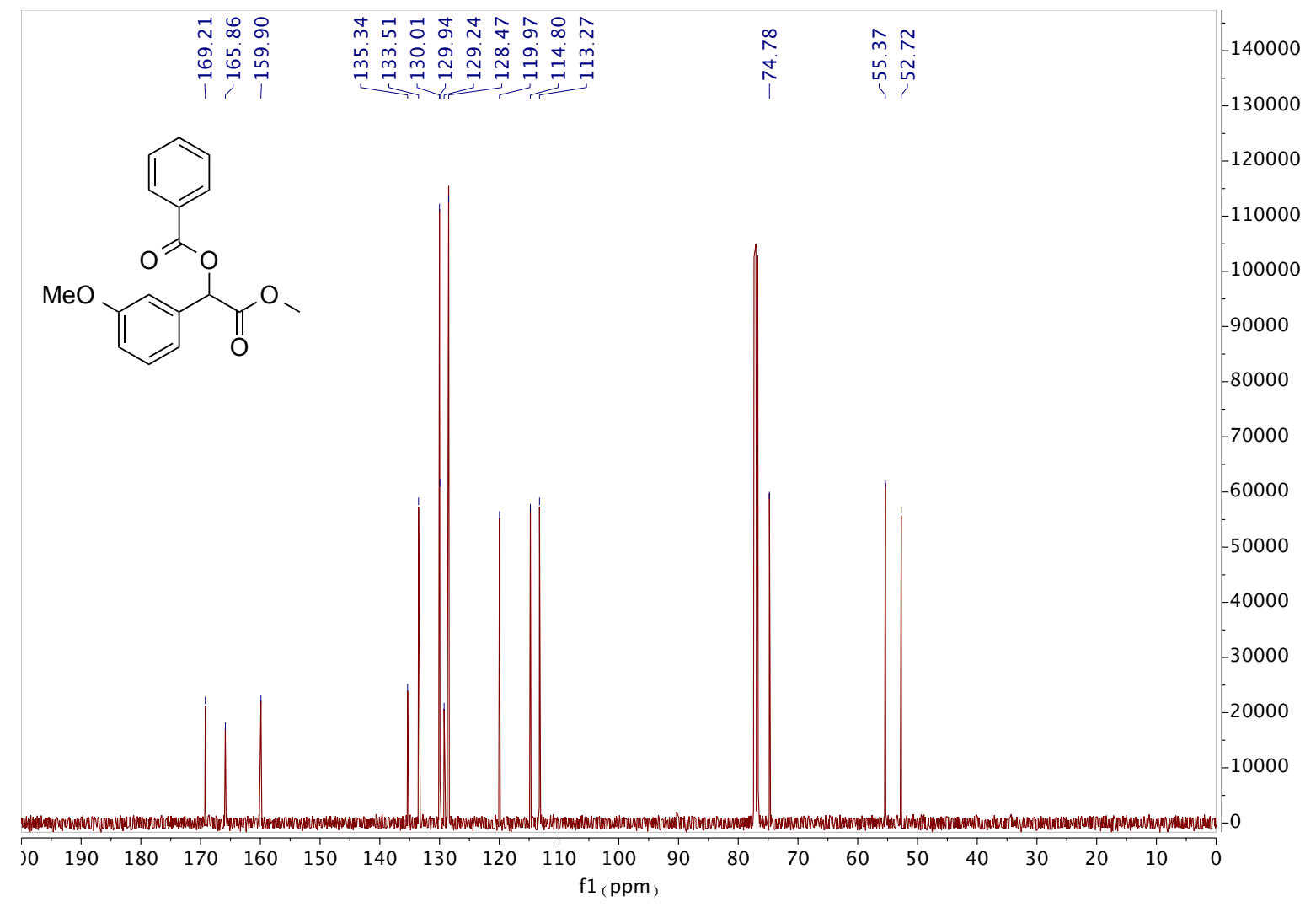




\section{1-(2-Chlorophenyl)-2-methoxy-2-oxoethyl benzoate (7l)}

${ }^{1}$ H NMR $(600 \mathrm{MHz}$, Chloroform- $d$ )

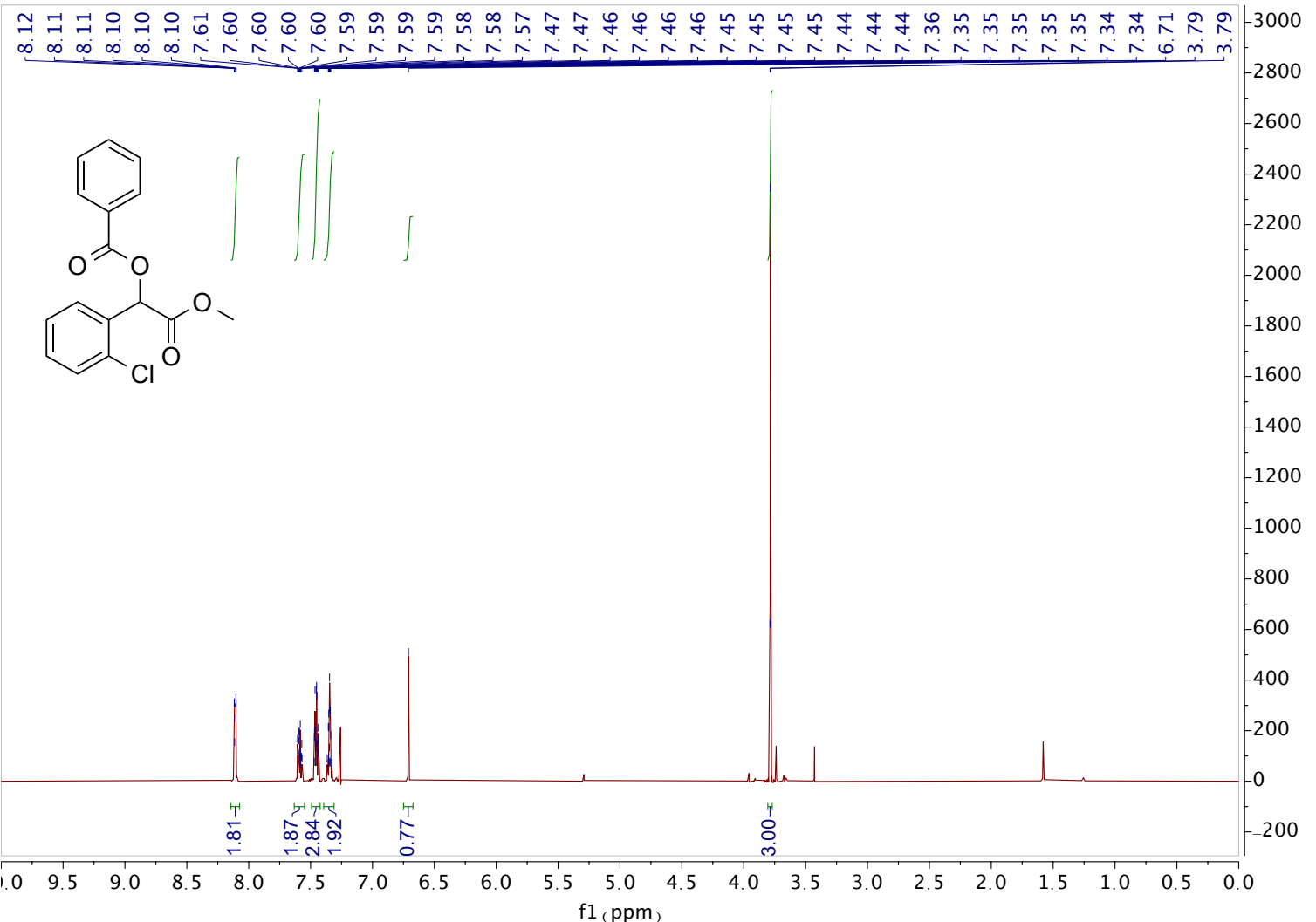

${ }^{13}$ C NMR (151 MHz, Chloroform- $d$ )

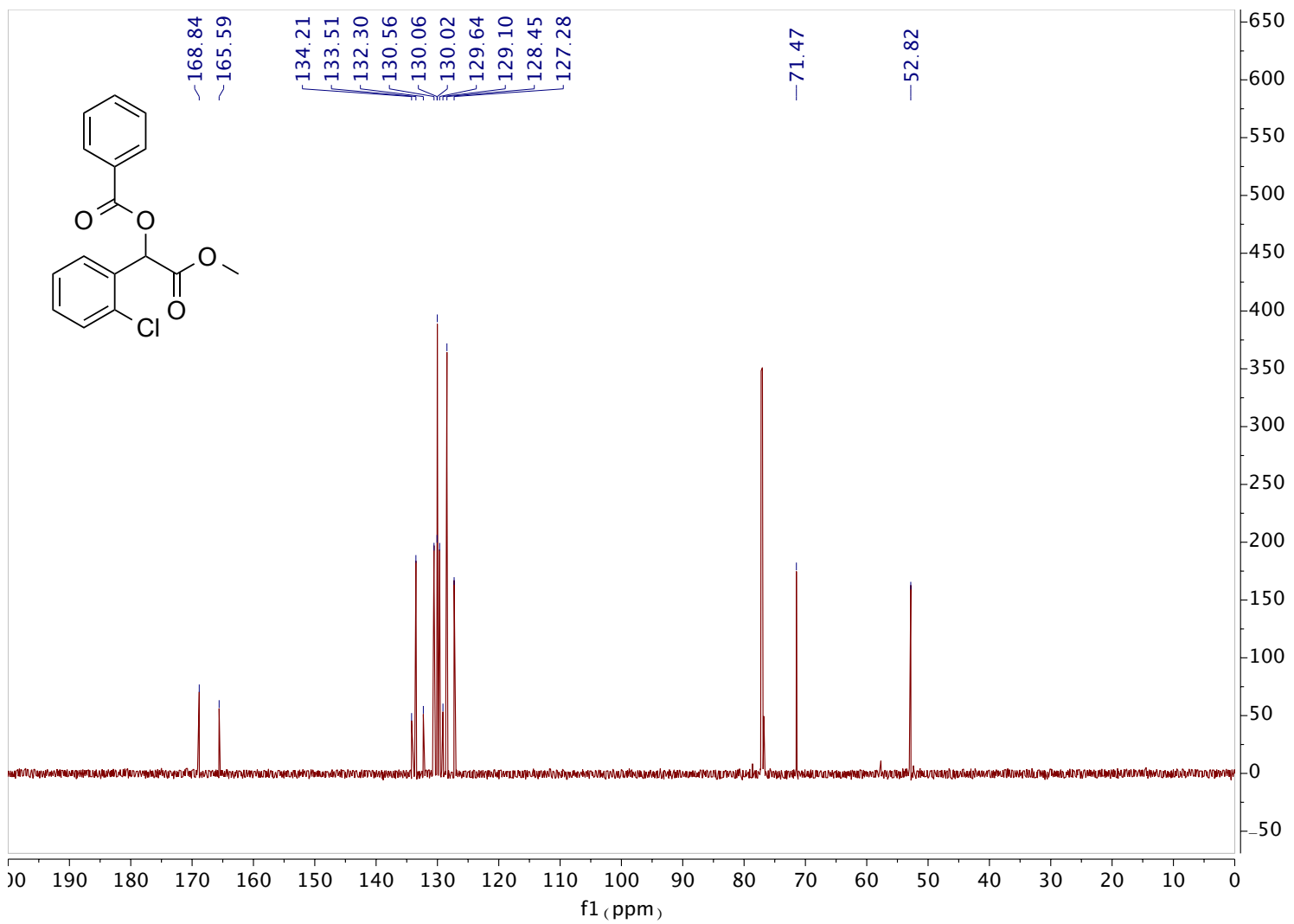


2-Ethoxy-2-oxo-1-(p-tolyl)ethyl benzoate $(7 \mathrm{~m})$

${ }^{1}$ H NMR (600 MHz, Chloroform- $d$ )

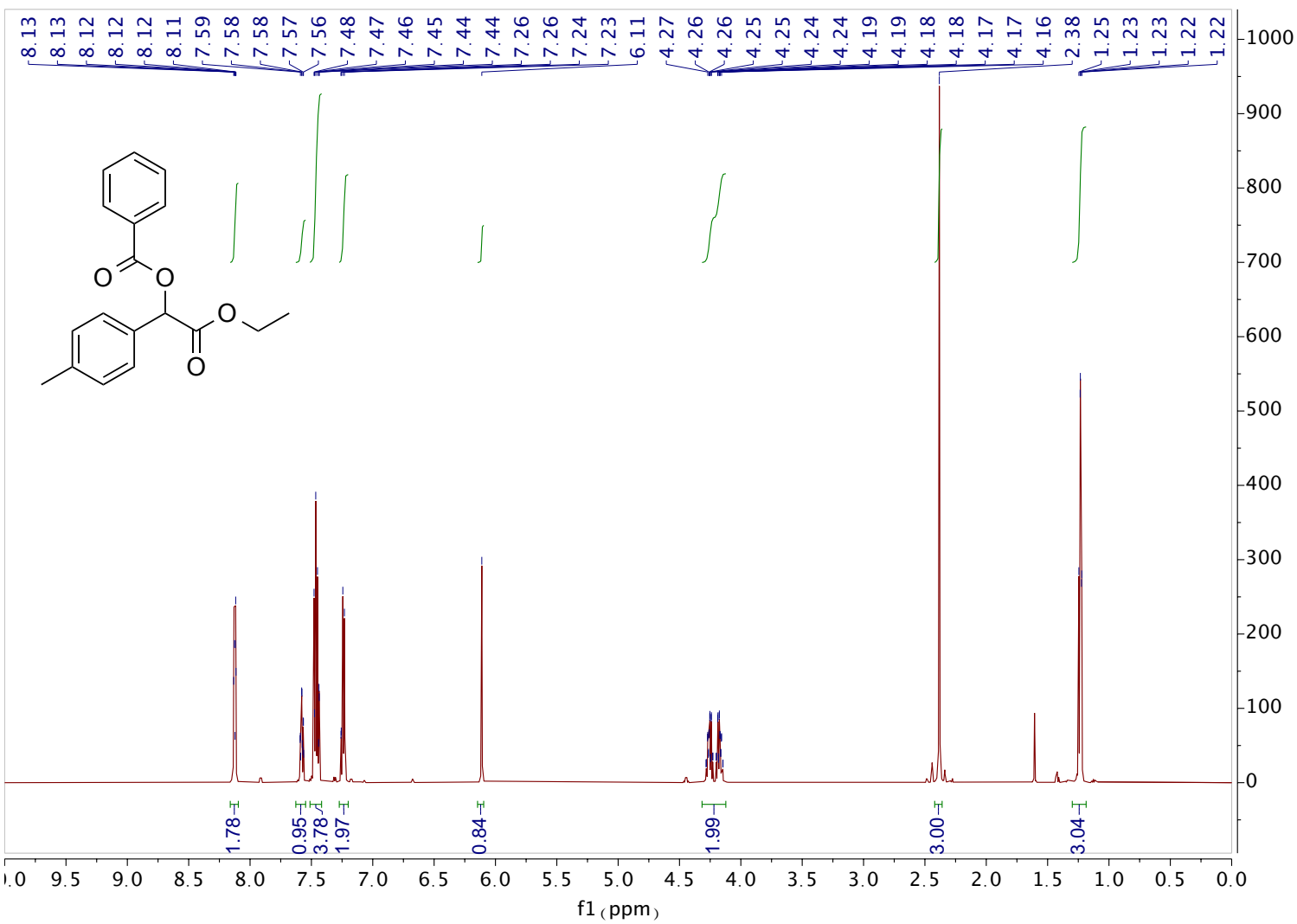

${ }^{13}$ C NMR (151 MHz, Chloroform- $d$ )

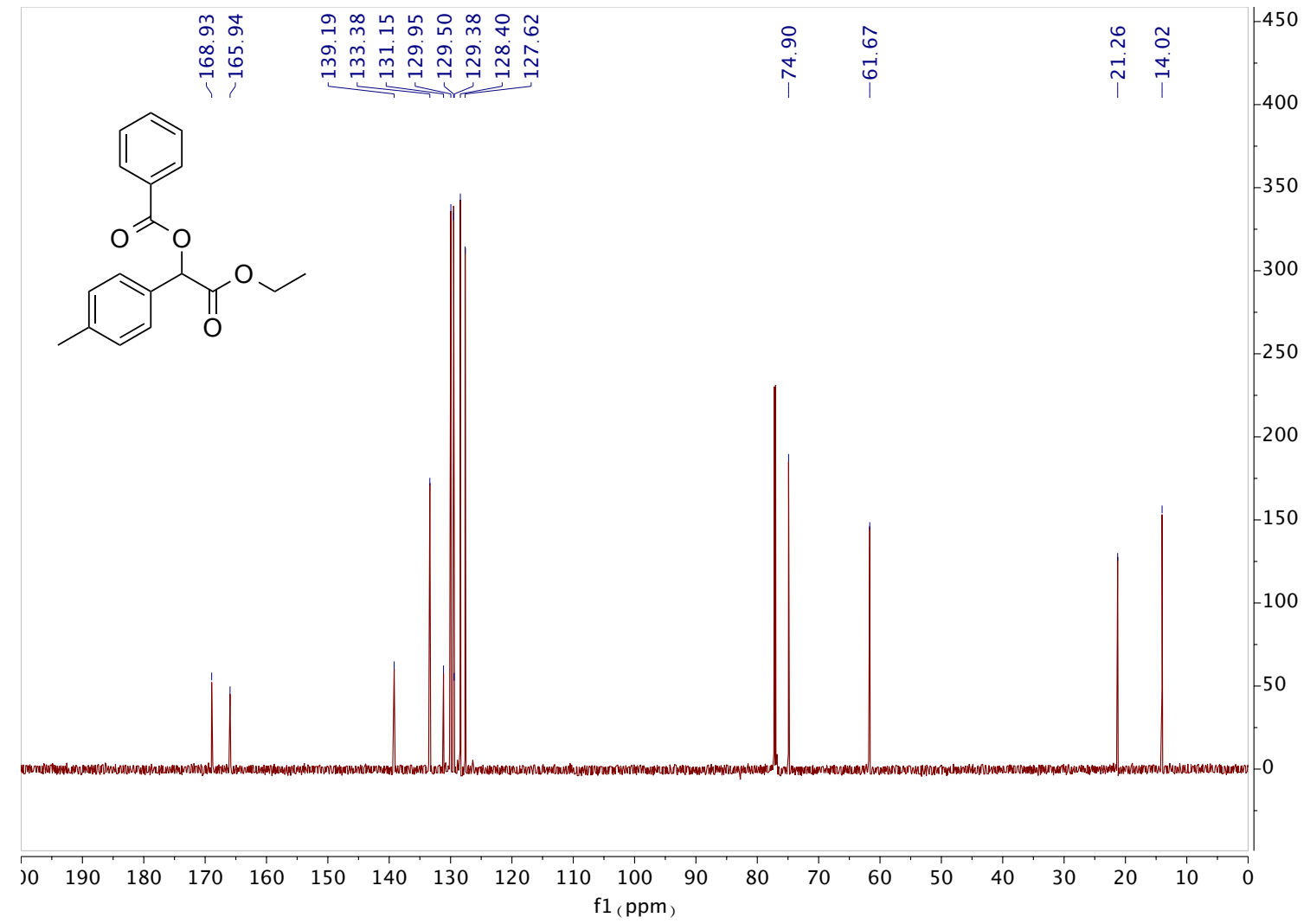


2-Methoxy-2-oxo-1-phenylethyl 4-methylbenzoate (7n)

${ }^{1}$ H NMR (600 MHz, Chloroform- $d$ )

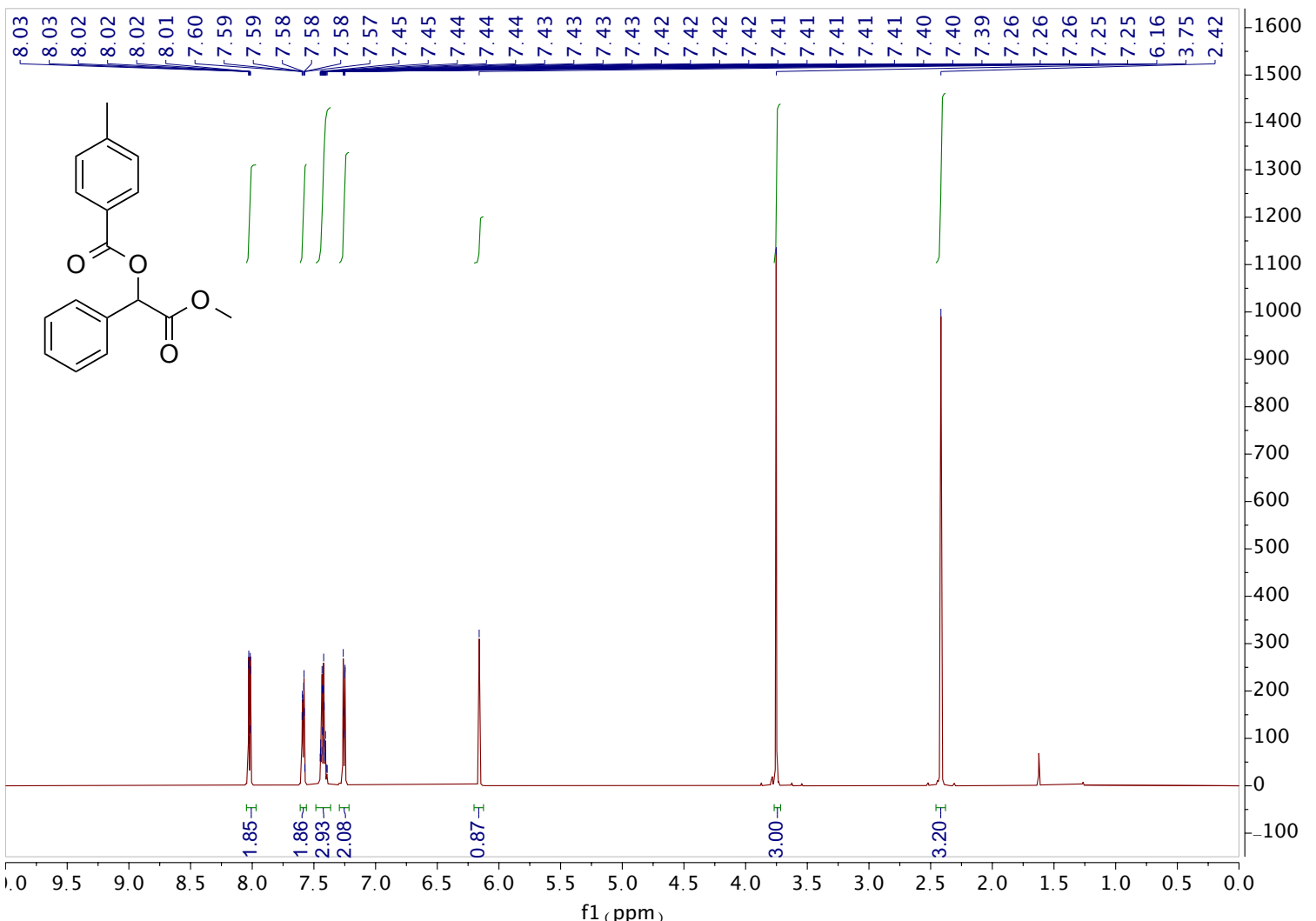

${ }^{13}$ C NMR (151 MHz, Chloroform- $d$ )

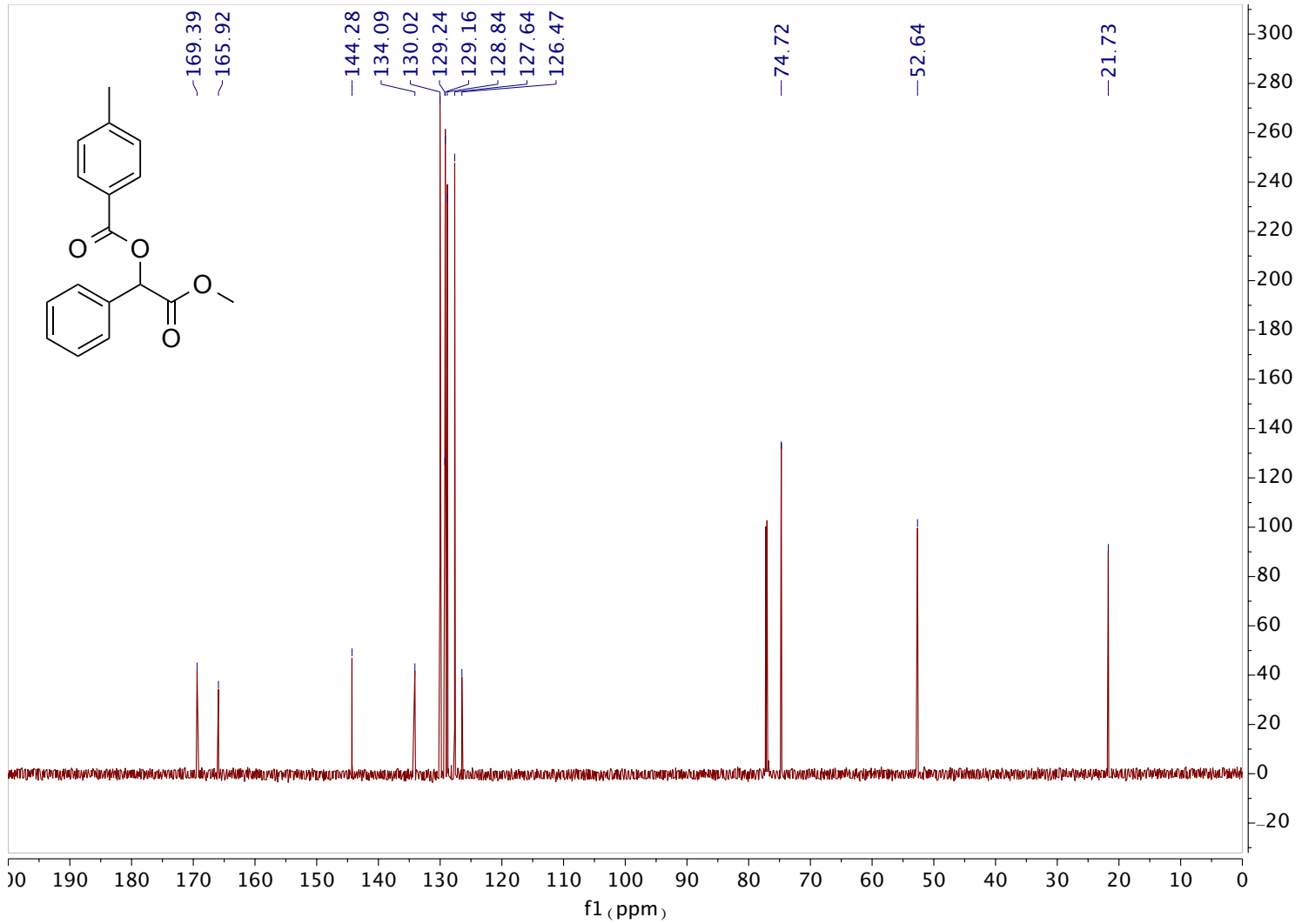


${ }^{1}$ H NMR (600 MHz, Chloroform- $d$ )

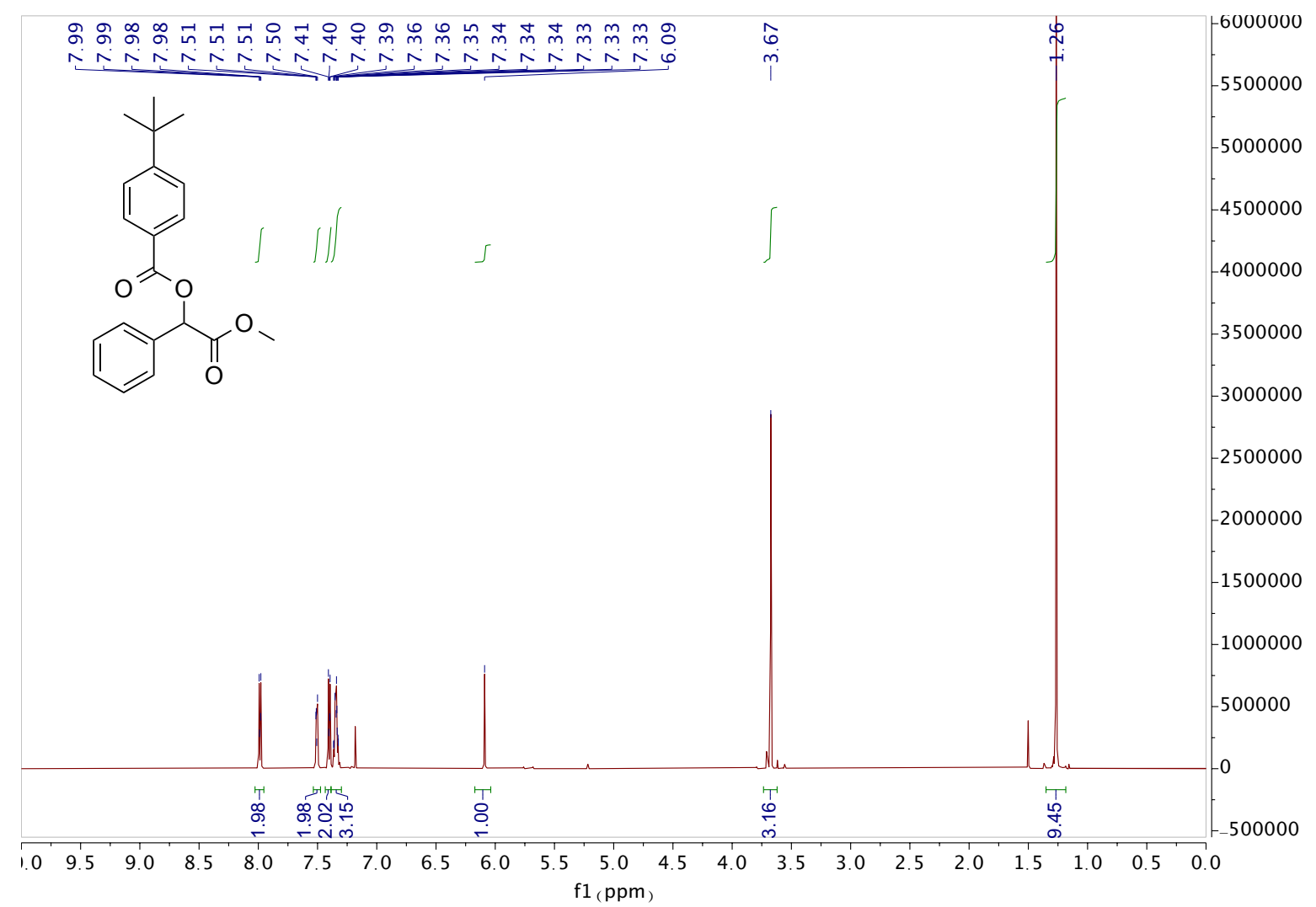

${ }^{13}$ C NMR (151 MHz, Chloroform- $d$ )

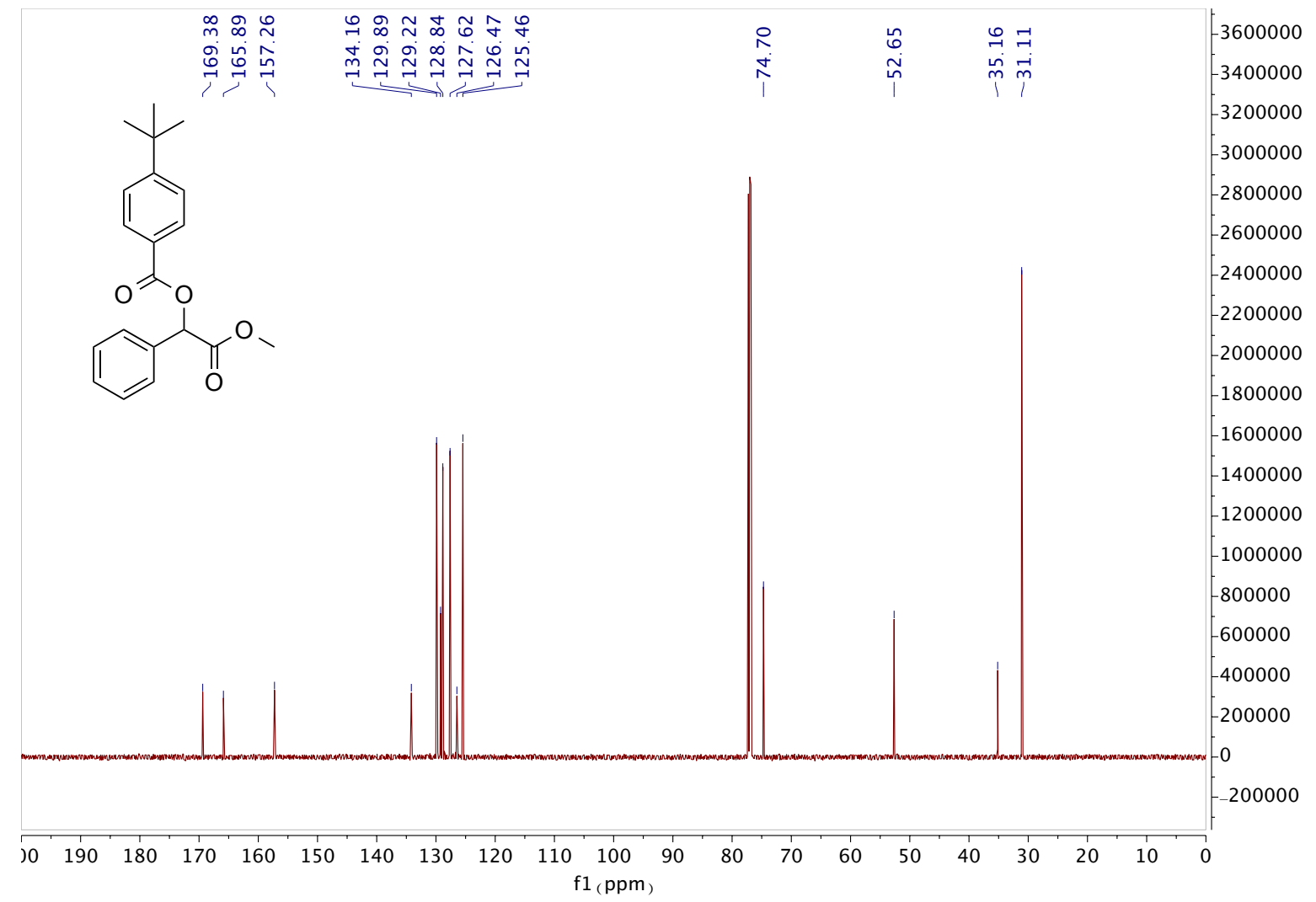


${ }^{1}$ H NMR (600 MHz, Chloroform- $d$ )

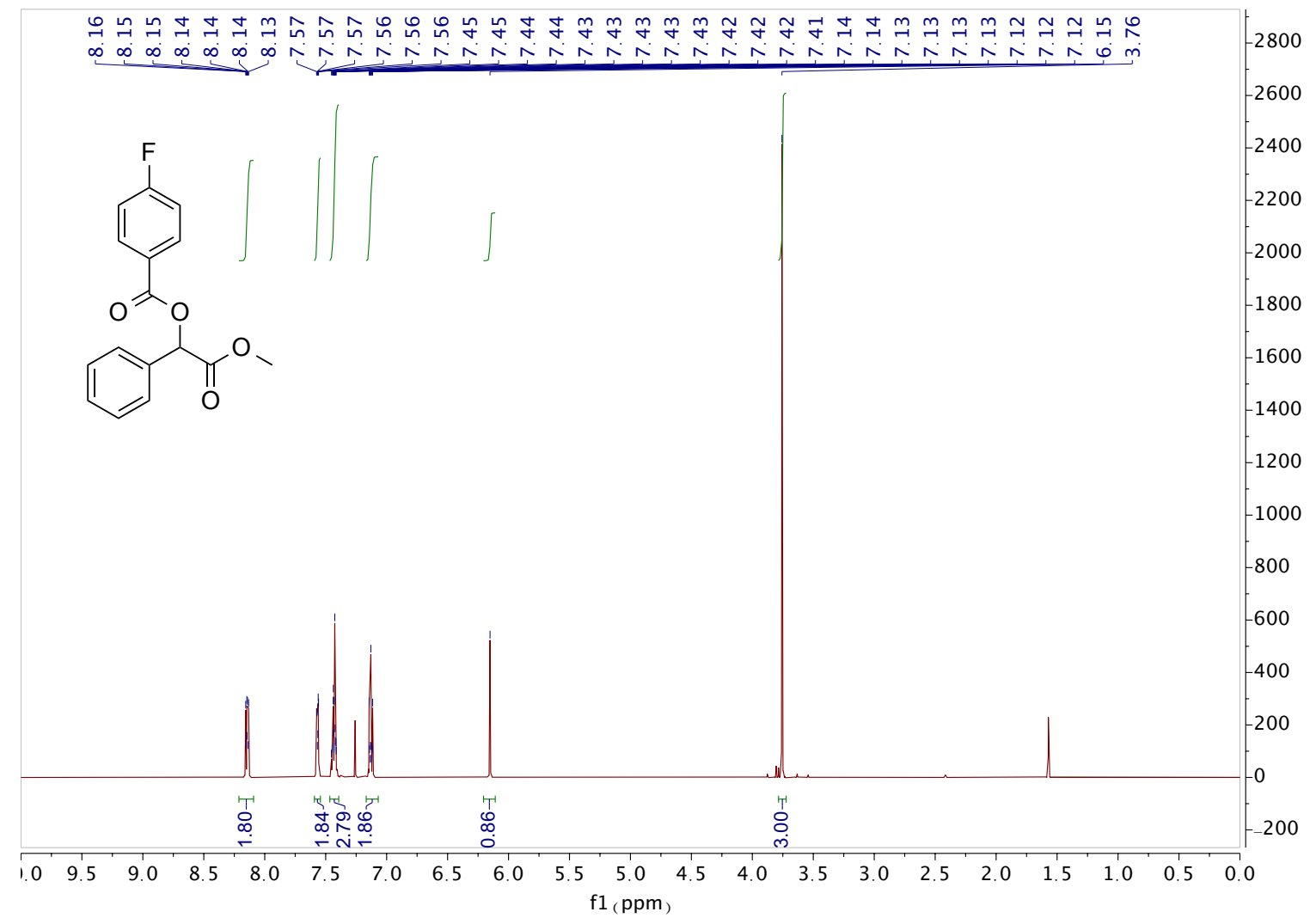

${ }^{13}$ C NMR (151 MHz, Chloroform- $d$ )

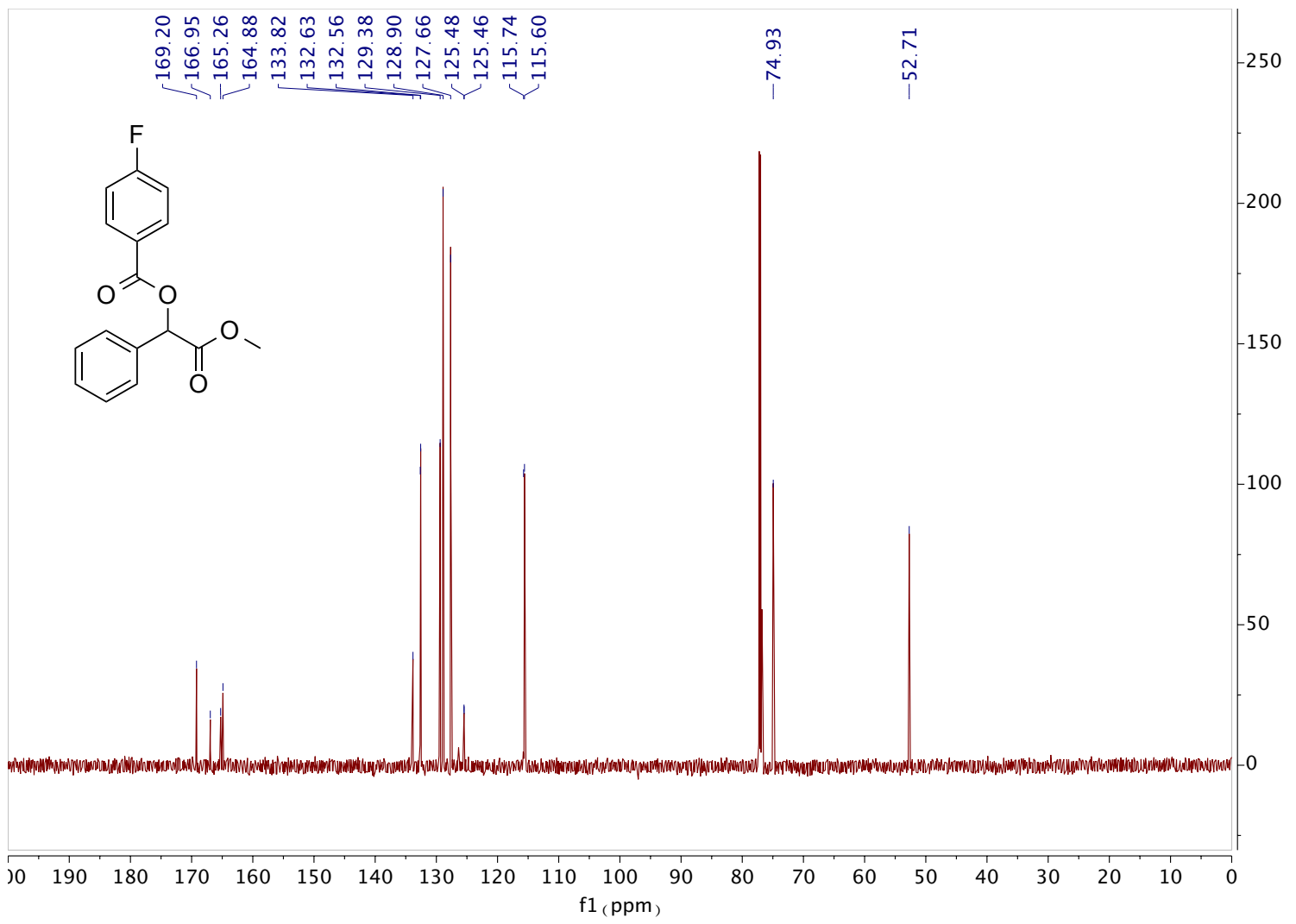


${ }^{19}$ F NMR (565 MHz, Chloroform- $d$ )

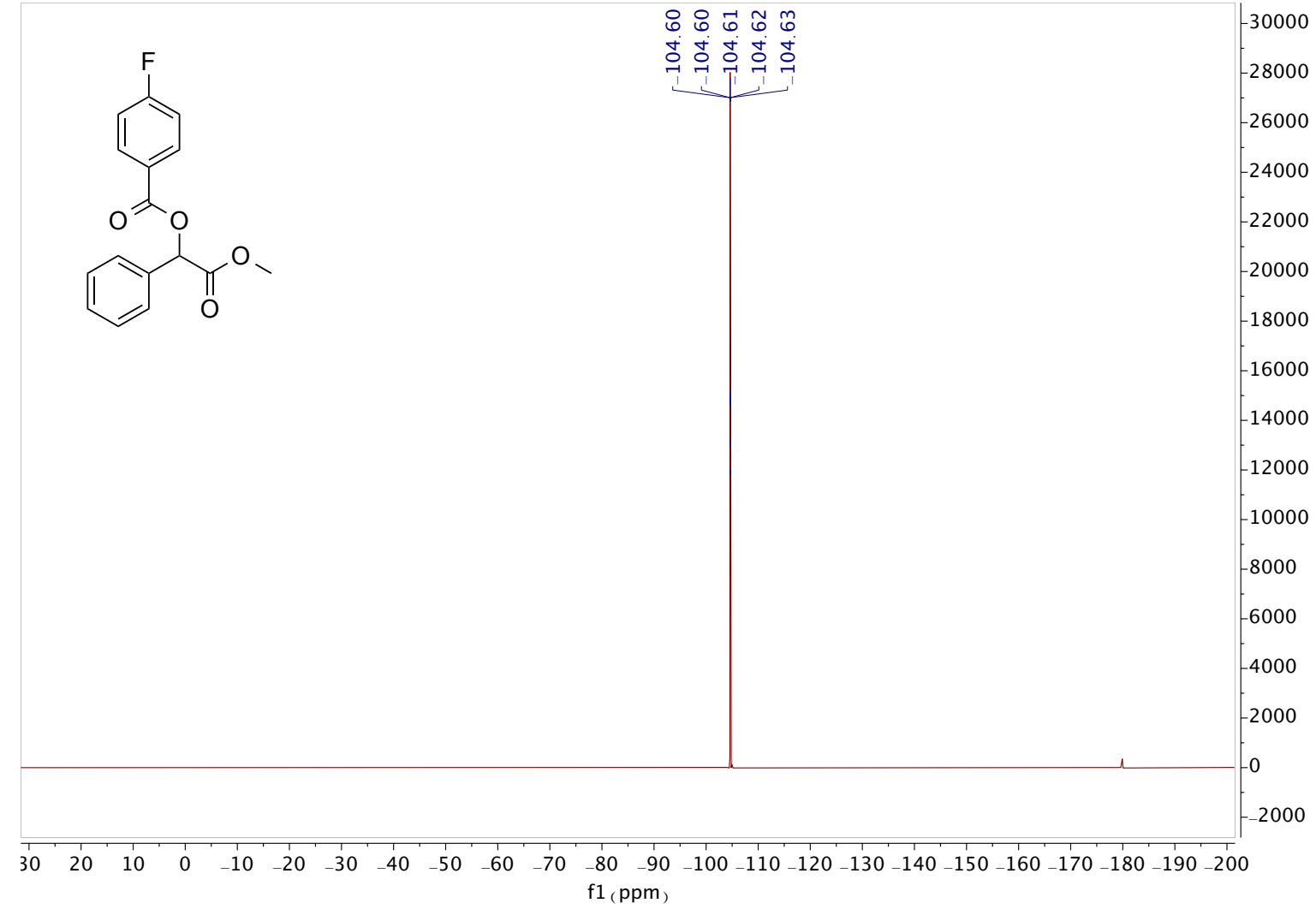


2-Methoxy-2-oxo-1-phenylethyl 3-methylbenzoate (7q)

${ }^{1}$ H NMR (600 MHz, Chloroform- $d$ )

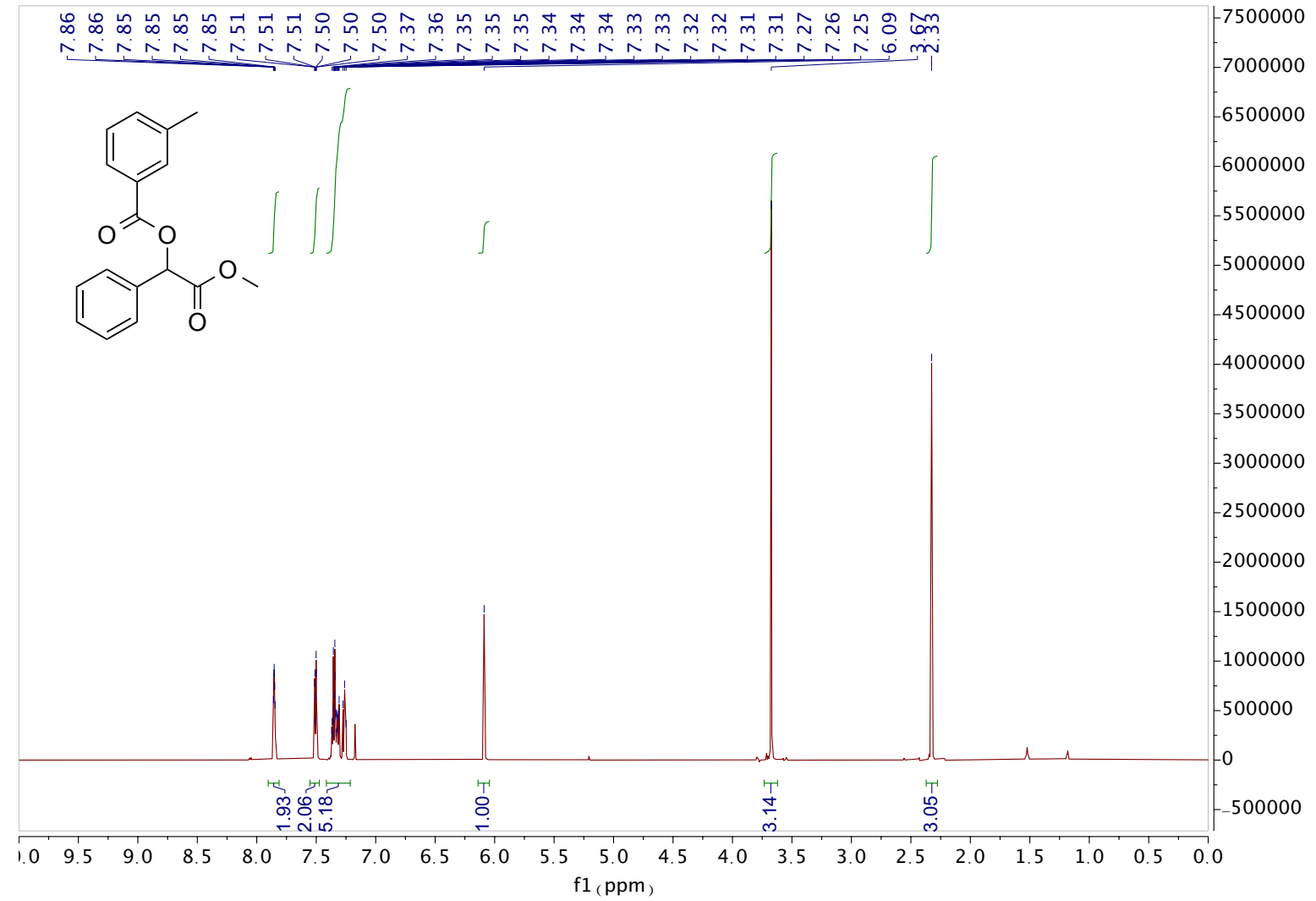

${ }^{13}$ C NMR (151 MHz, Chloroform- $d$ )

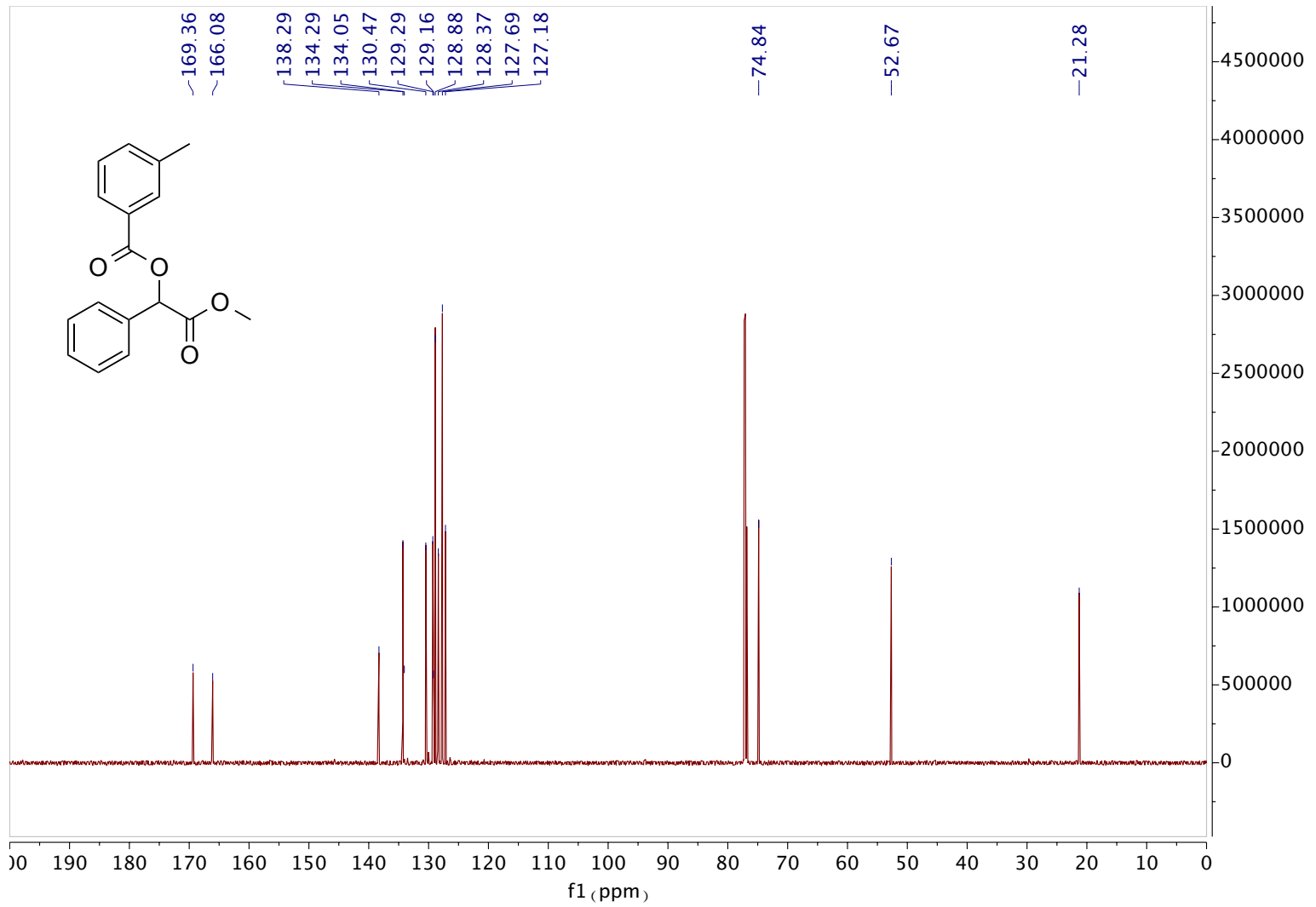


${ }^{1}$ H NMR (600 MHz, Chloroform- $d$ )

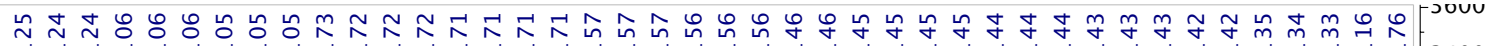

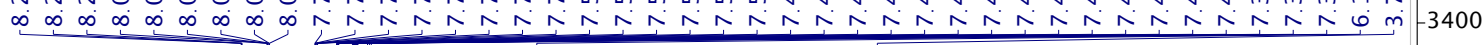

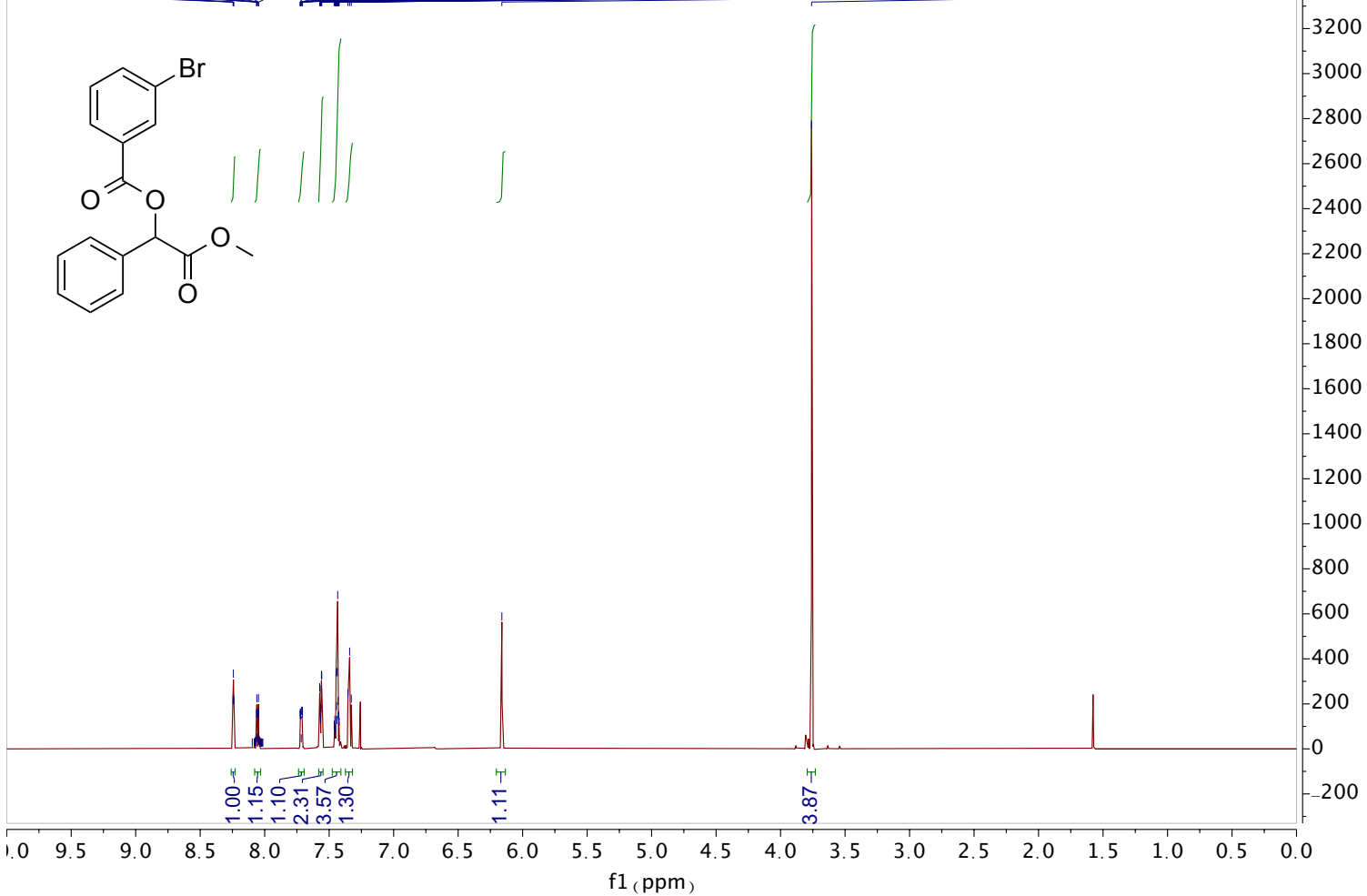

${ }^{13}$ C NMR (151 MHz, Chloroform- $d$ )

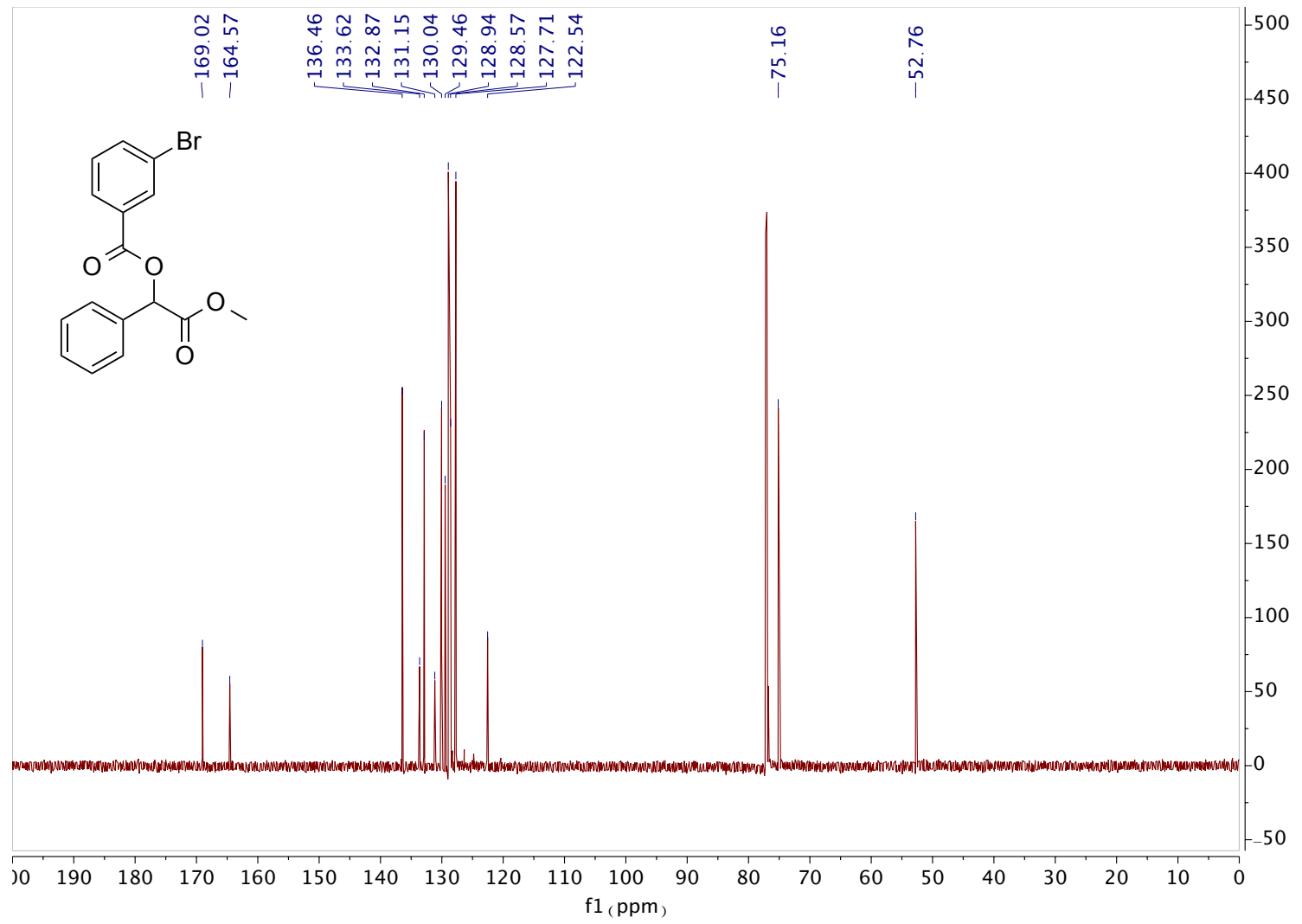


${ }^{1}$ H NMR (600 MHz, Chloroform- $d$

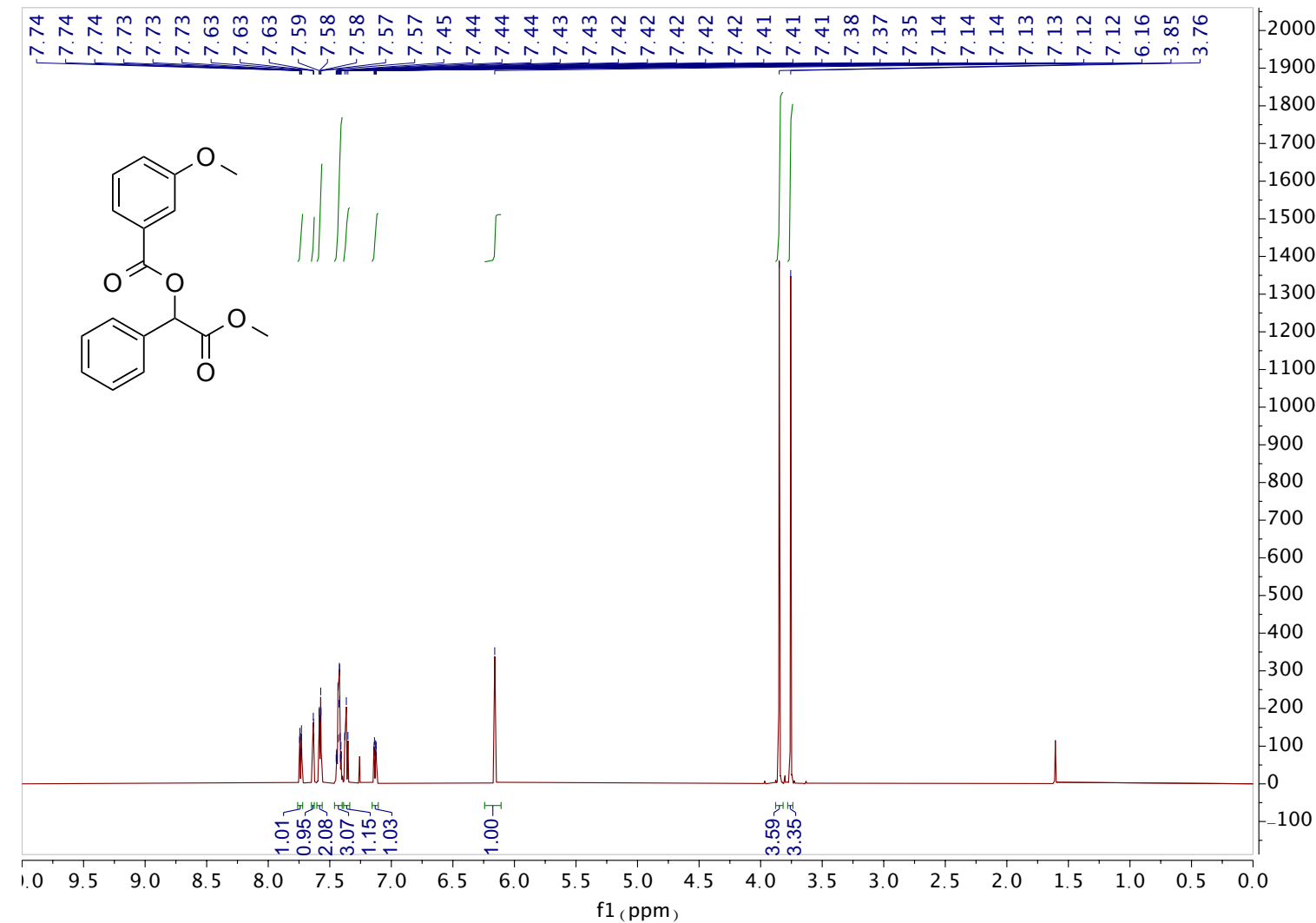

${ }^{13}$ C NMR (151 MHz, Chloroform- $d$ )

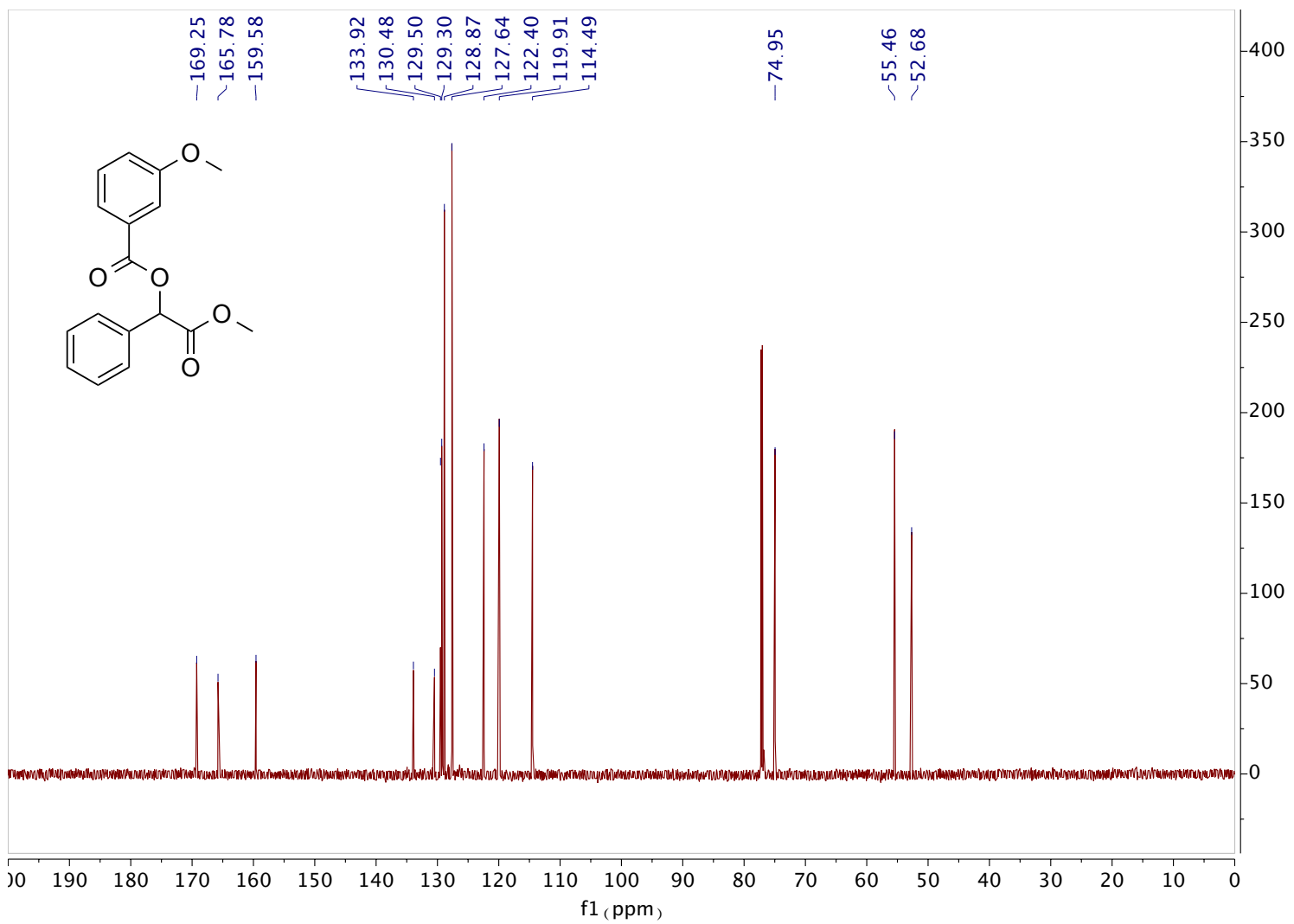


2-Methoxy-2-oxo-1-phenylethyl 2-methylbenzoate (7t)

${ }^{1}$ H NMR (600 MHz, Chloroform- $d$ )

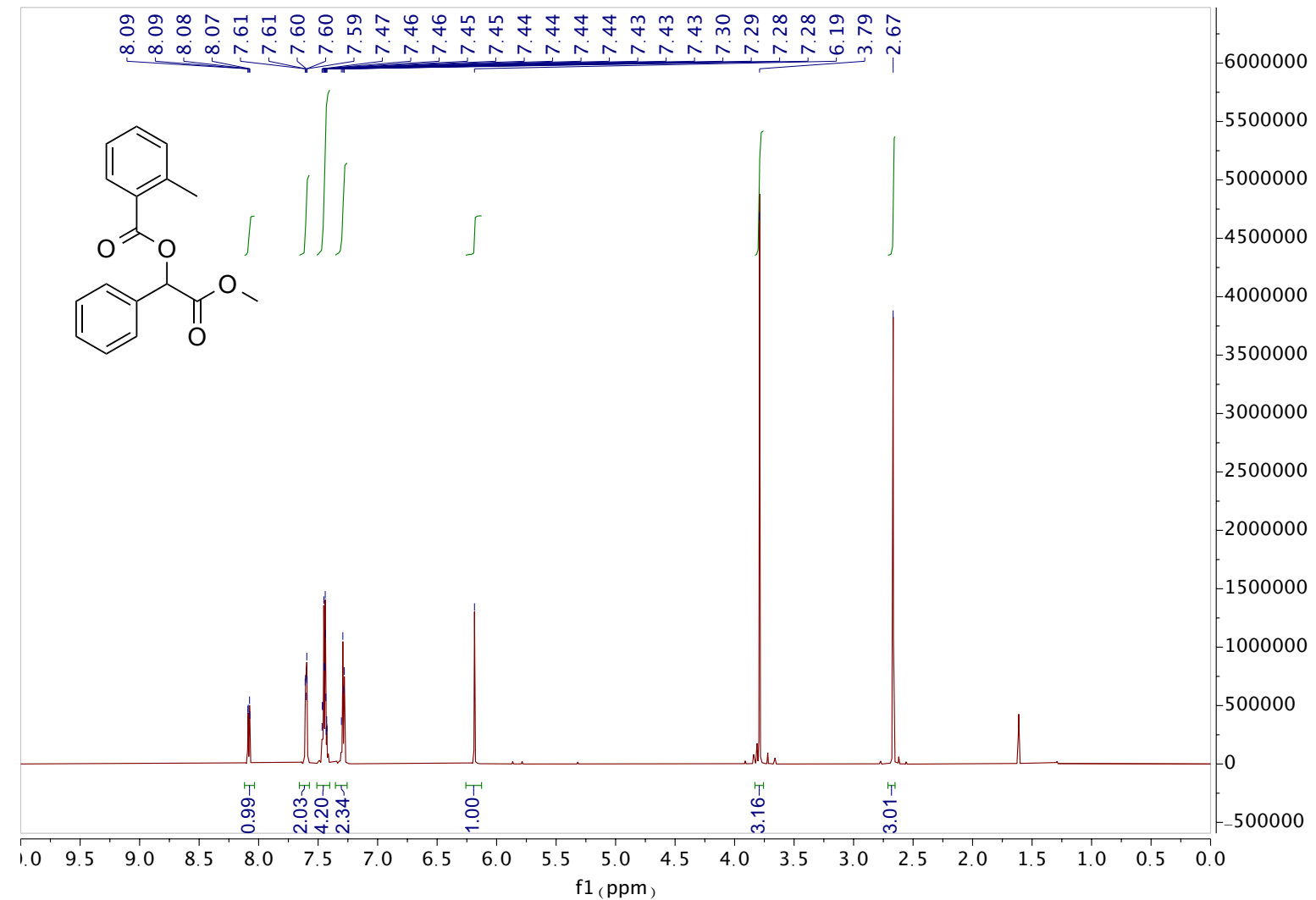

${ }^{13}$ C NMR (151 MHz, Chloroform- $d$ )

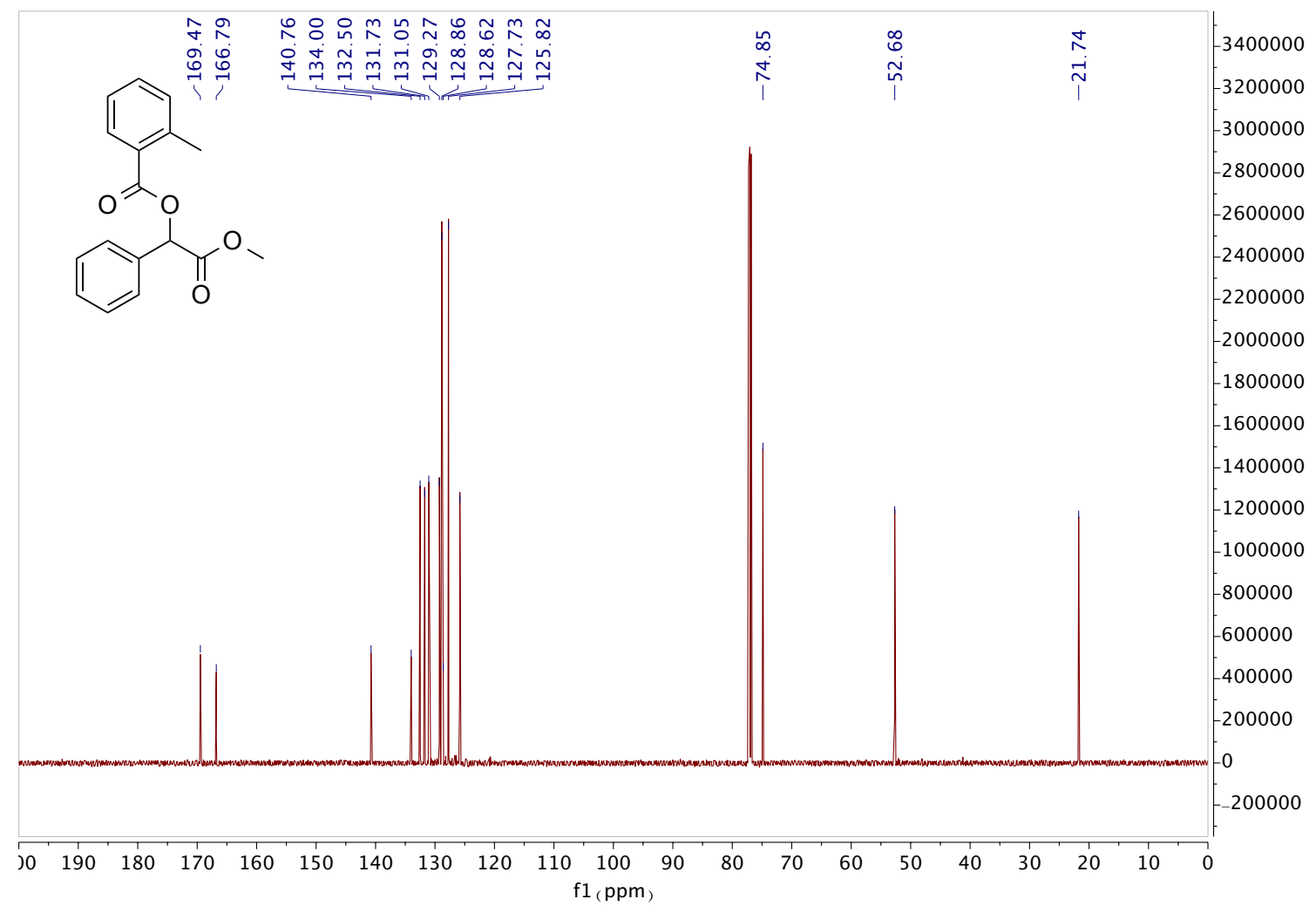


2-Methoxy-2-oxo-1-phenylethyl 2-fluorobenzoate (7u)

${ }^{1}$ H NMR (600 MHz, Chloroform- $d$ )

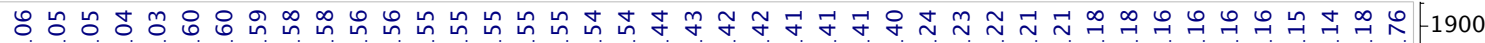

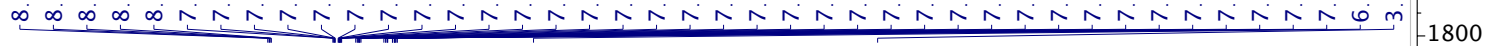

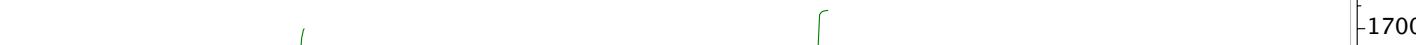<smiles>O=C(O)c1ccccc1F</smiles>

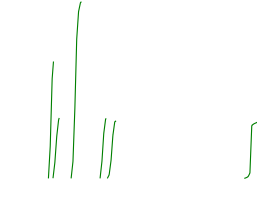<smiles>COC(=O)C(C)c1ccccc1</smiles>
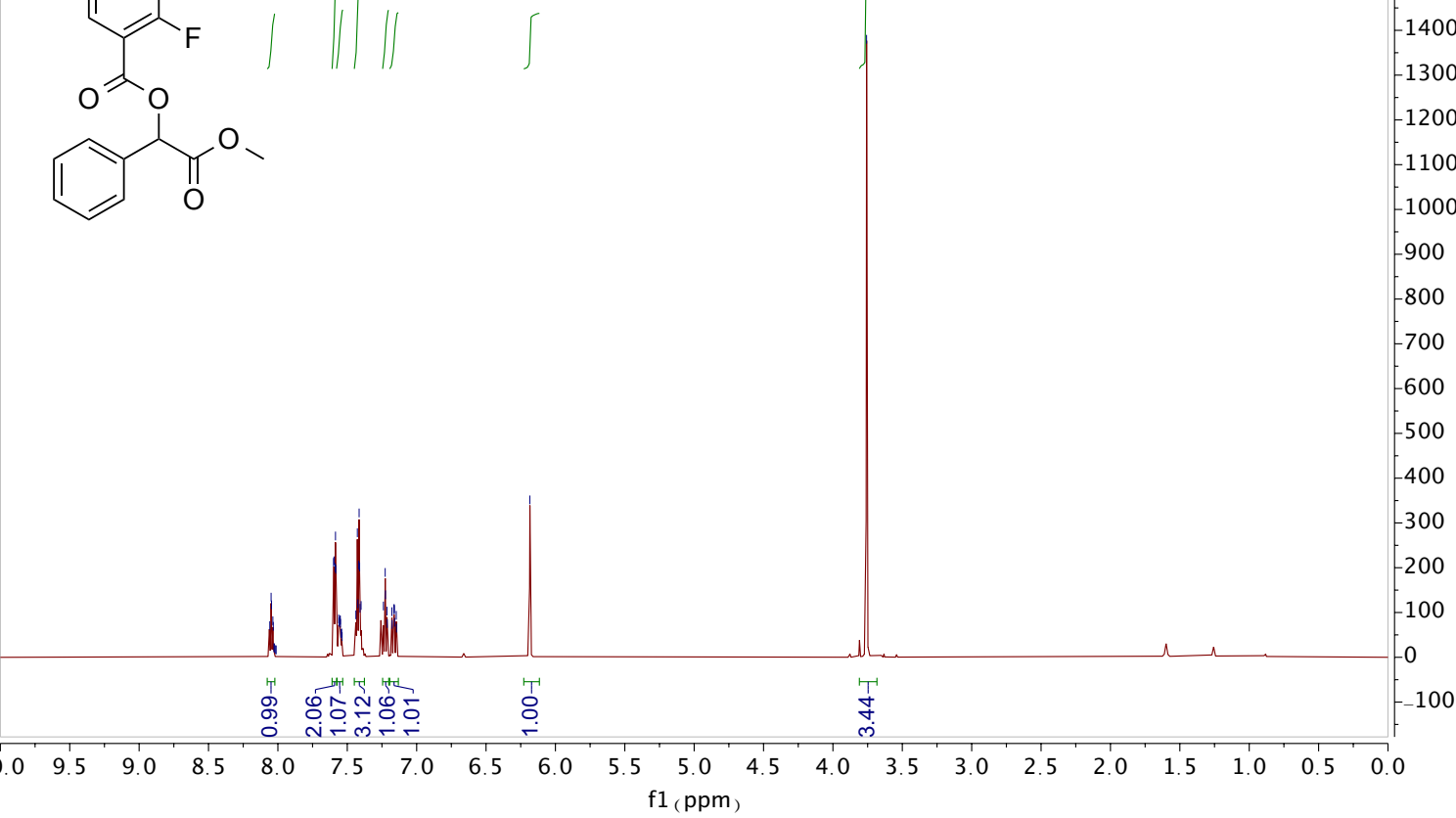

${ }^{13}$ C NMR (151 MHz, Chloroform- $d$ )

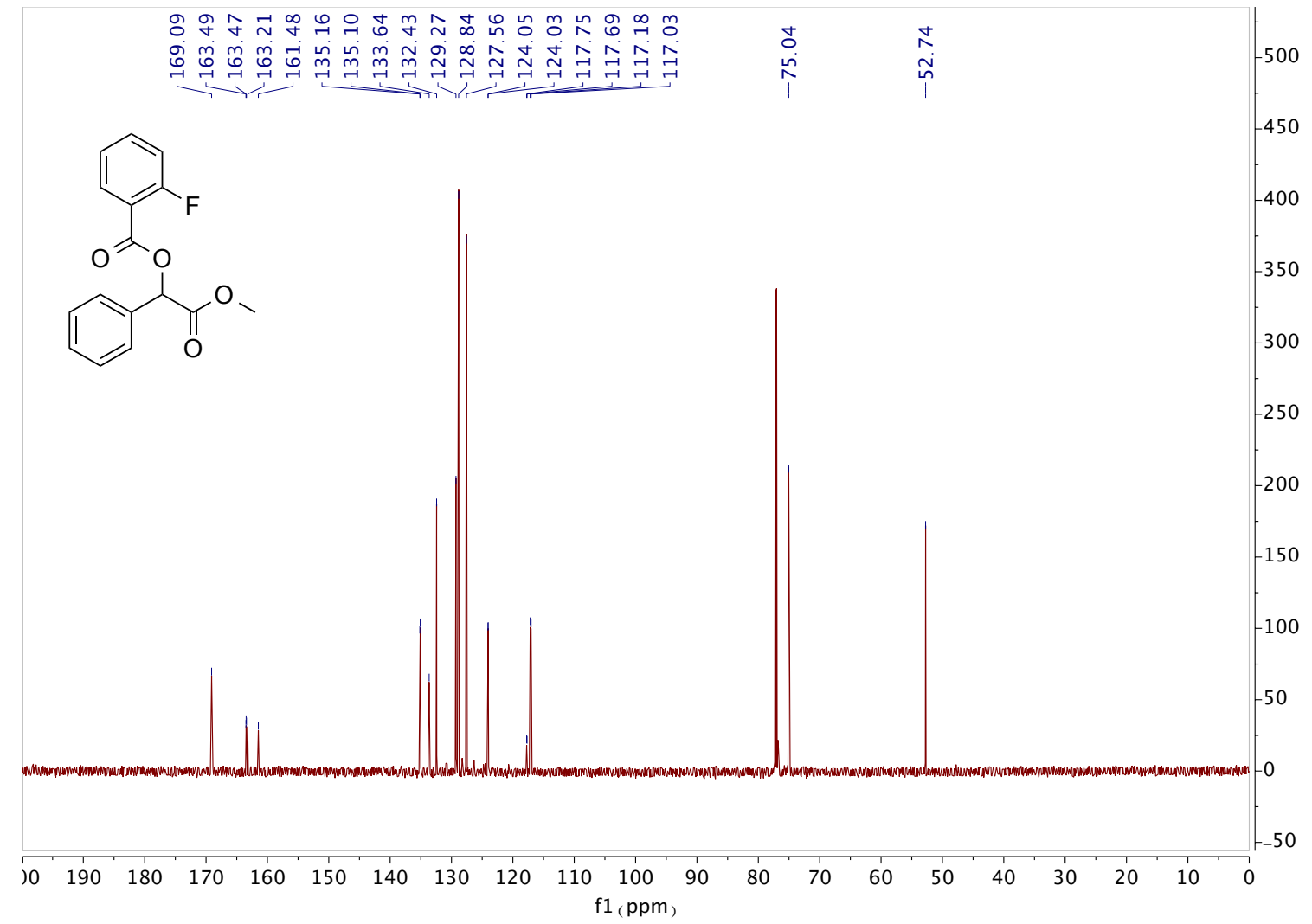


${ }^{19}$ F NMR (565 MHz, Chloroform-d)

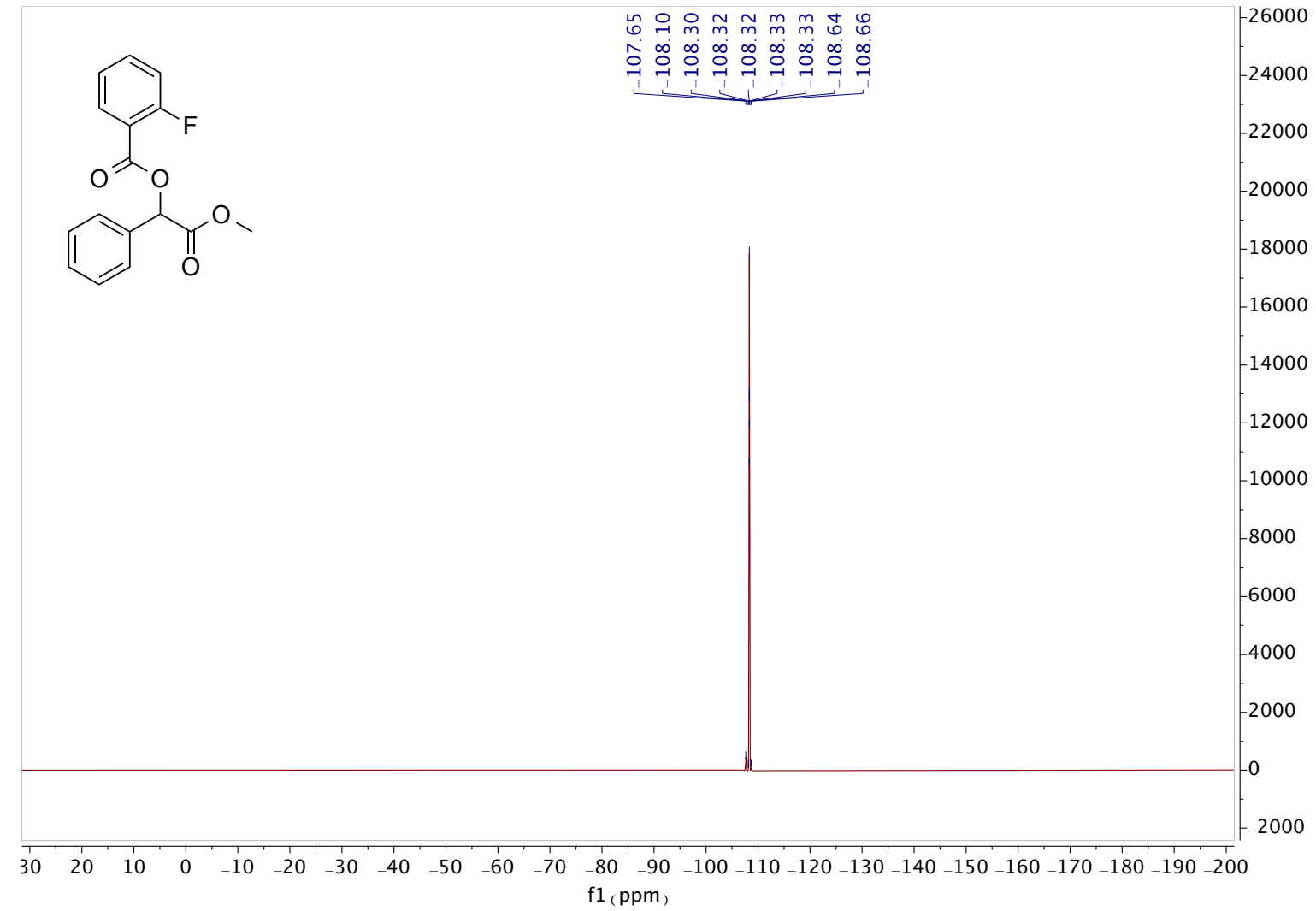


${ }^{1}$ H NMR (600 MHz, Chloroform- $d$ )

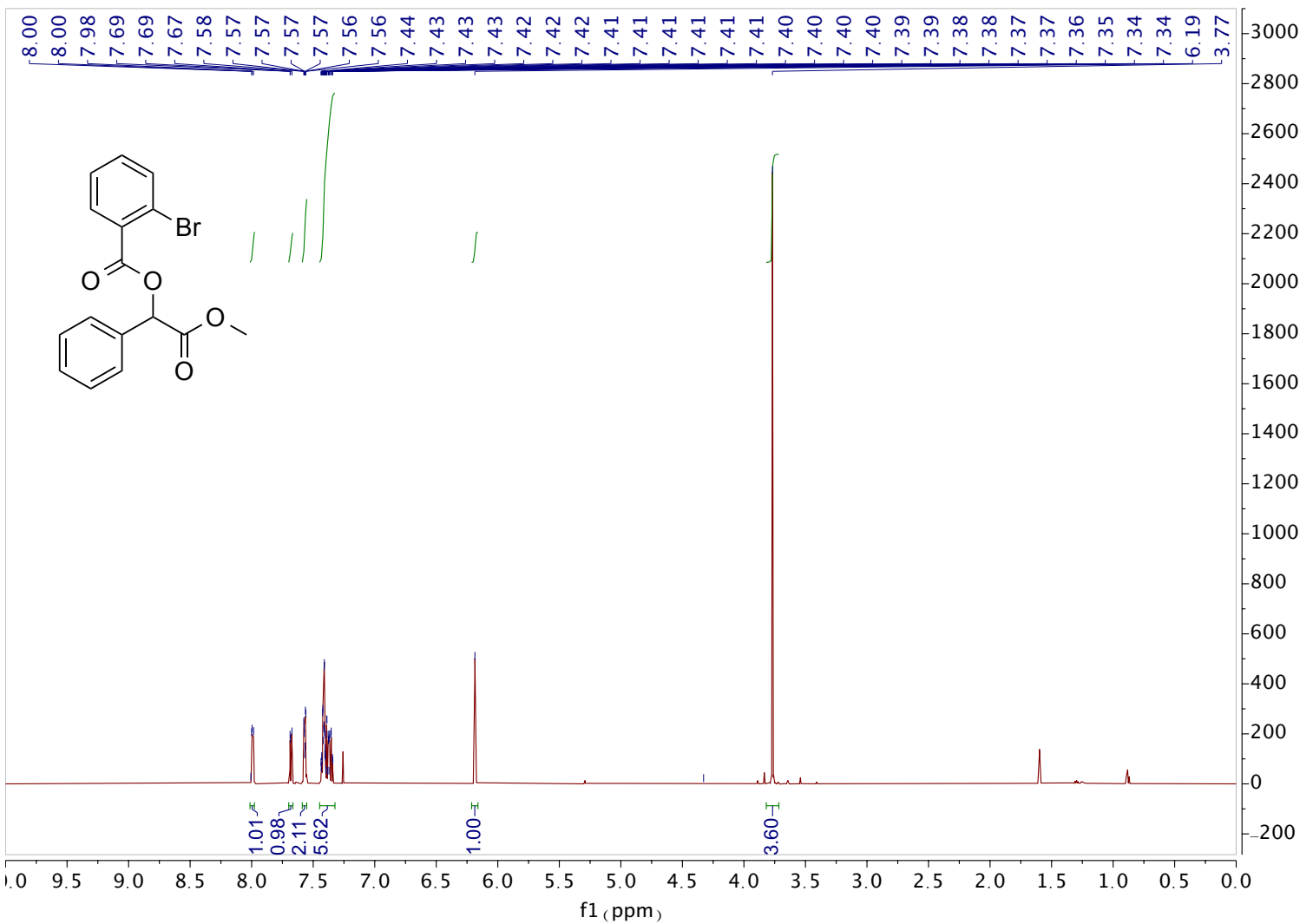

${ }^{13}$ C NMR (151 MHz, Chloroform- $d$ )

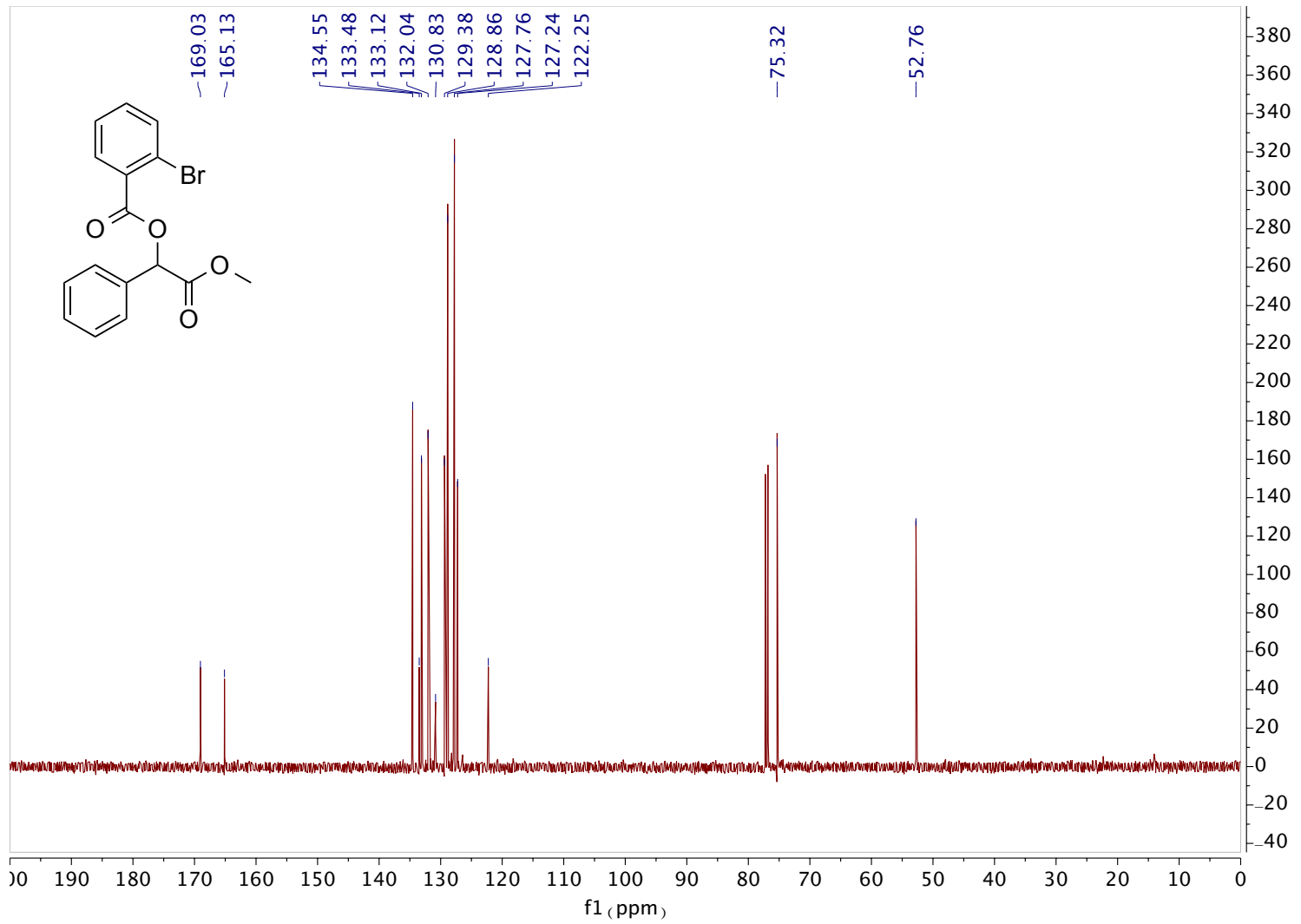


${ }^{1}$ H NMR (600 MHz, Chloroform- $d$ )

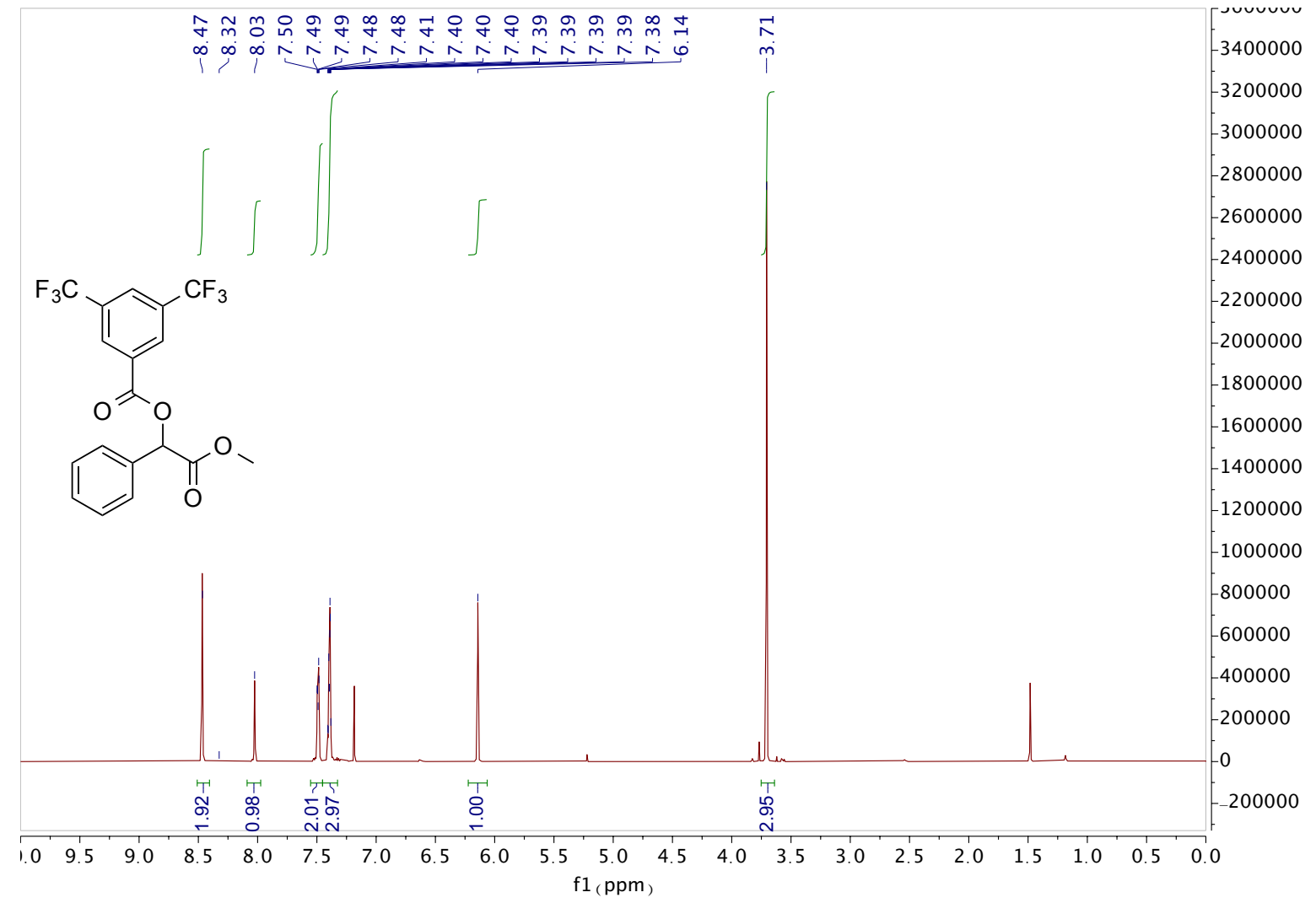

${ }^{13}$ C NMR (151 MHz, Chloroform- $d$ )

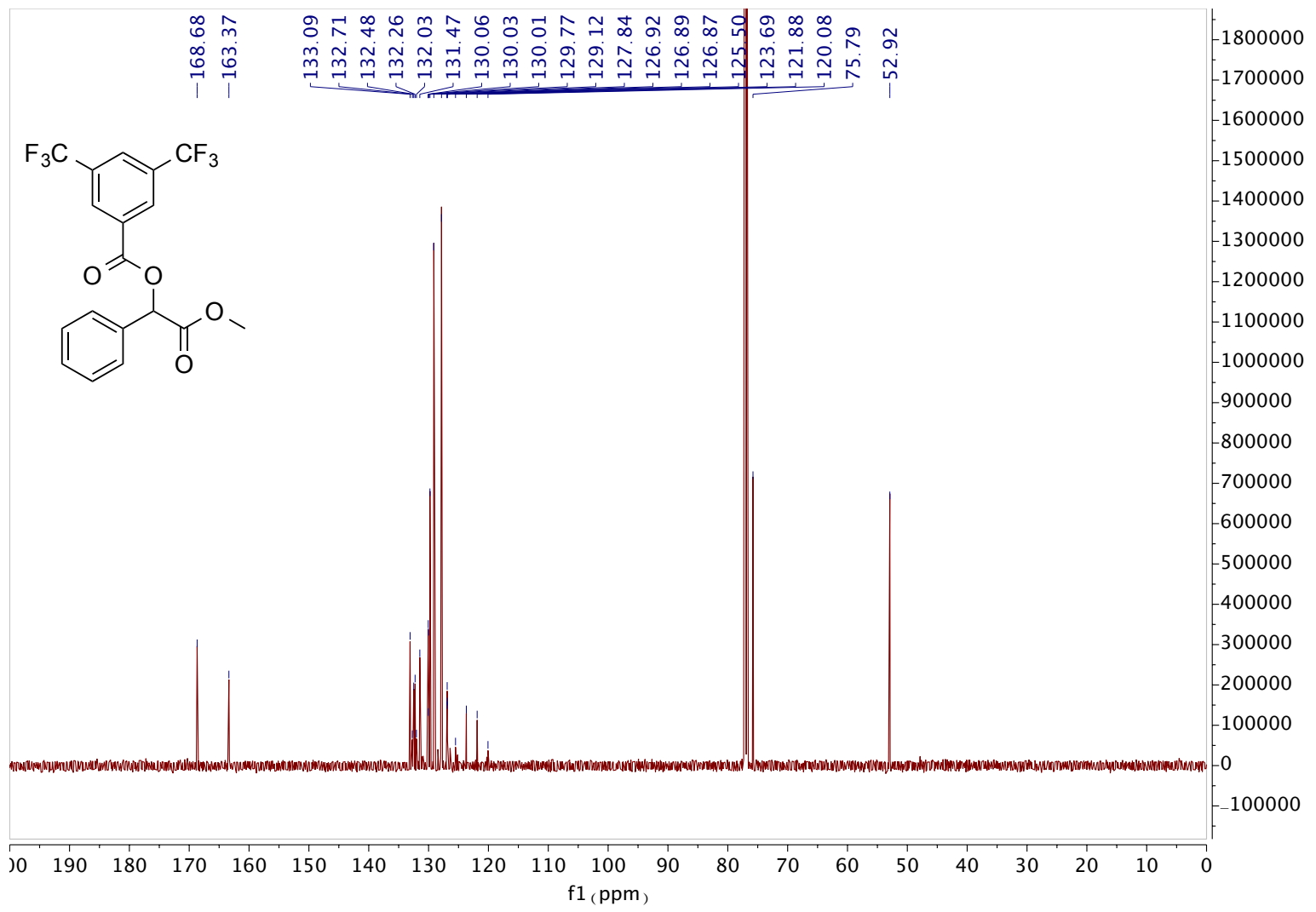


${ }^{19}$ F NMR (565 MHz, Chloroform- $d$ )

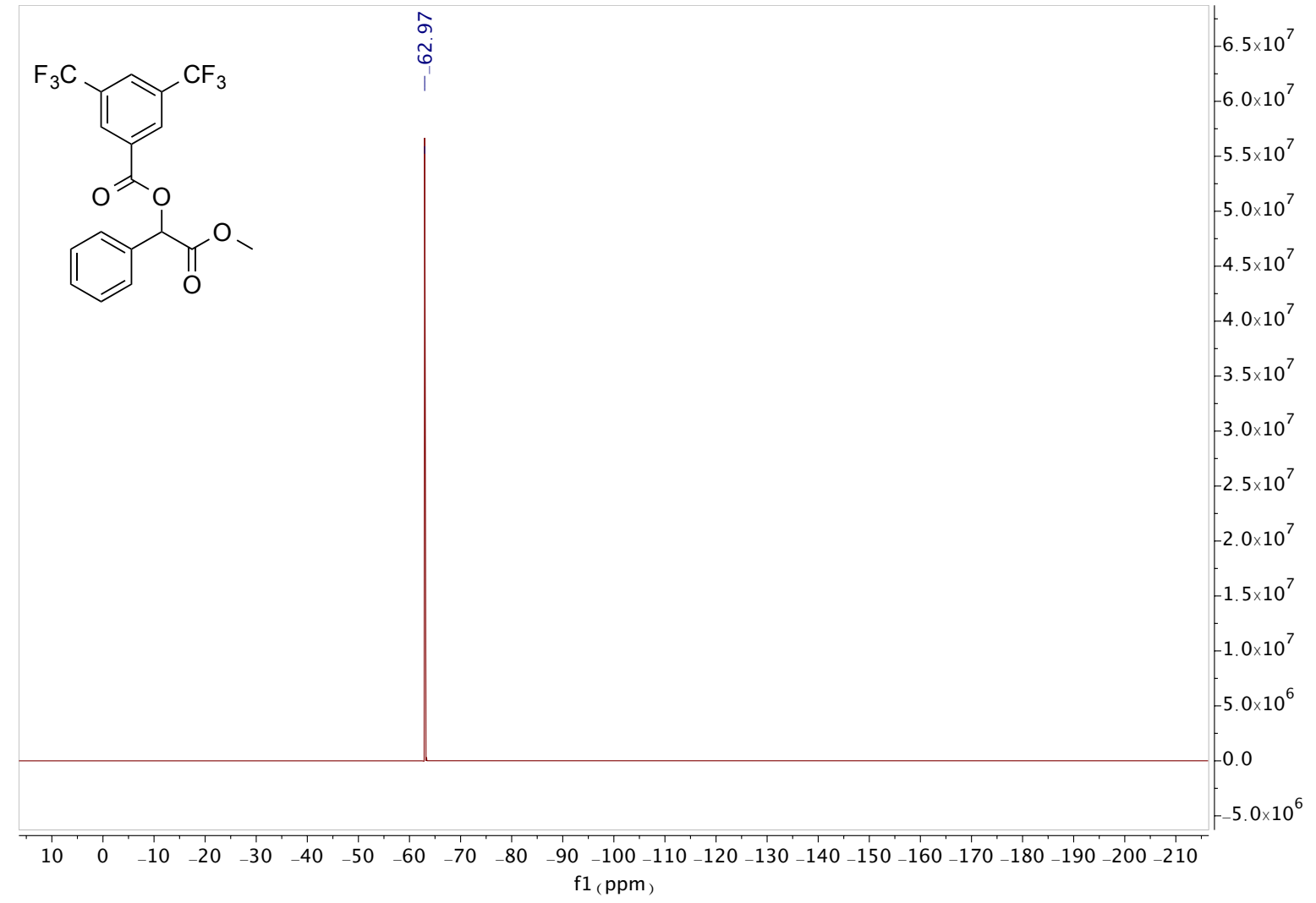




\section{2-Methoxy-2-oxo-1-phenylethyl 2-naphthoate (7x)}

\section{${ }^{1}$ H NMR $(600 \mathrm{MHz}$, Chloroform- $d)$}

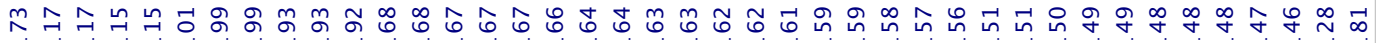

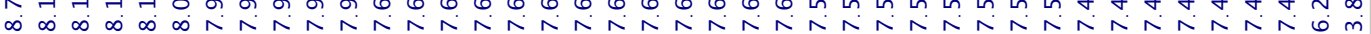

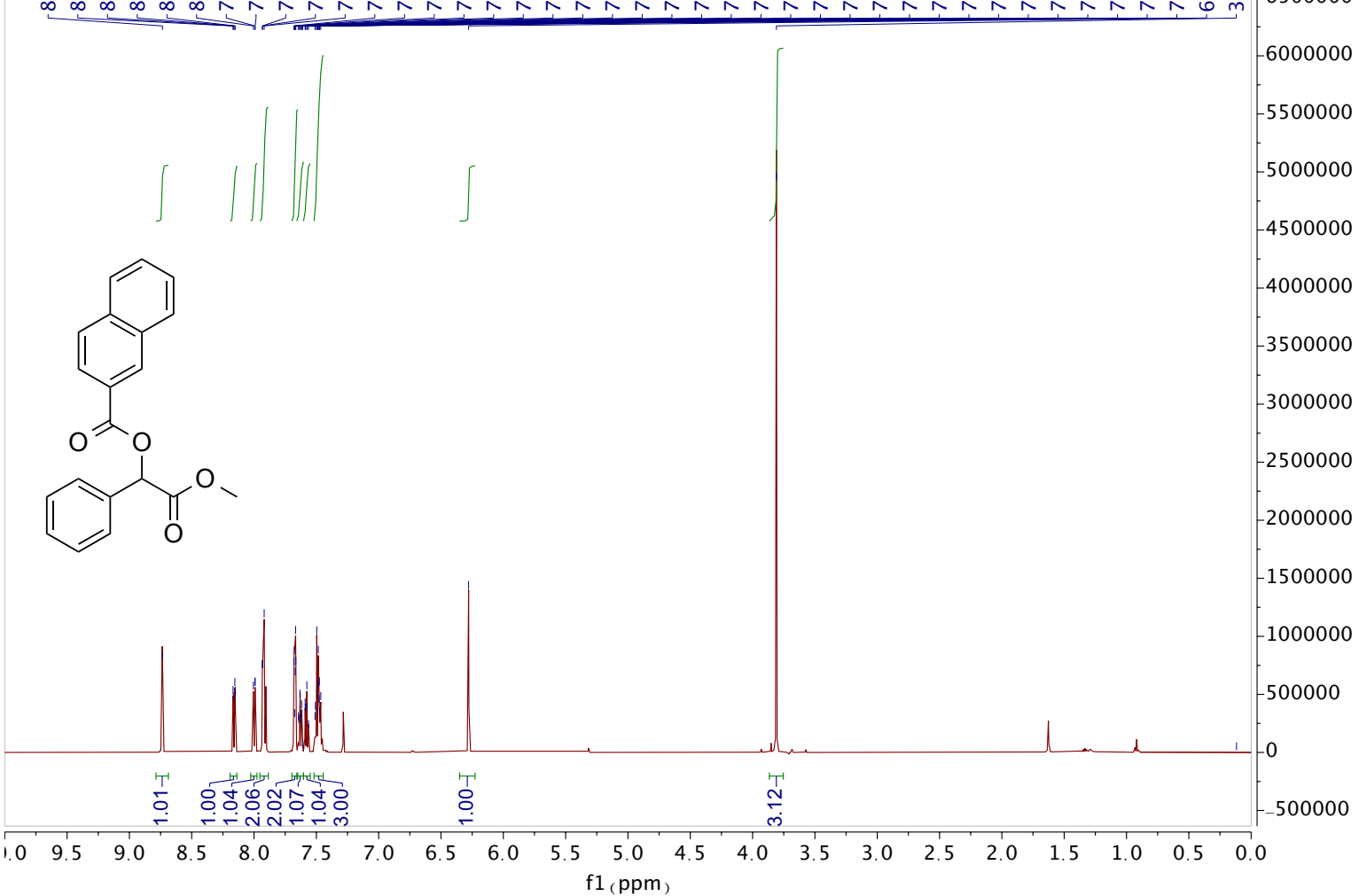

${ }^{13}$ C NMR (151 MHz, Chloroform- $d$ )

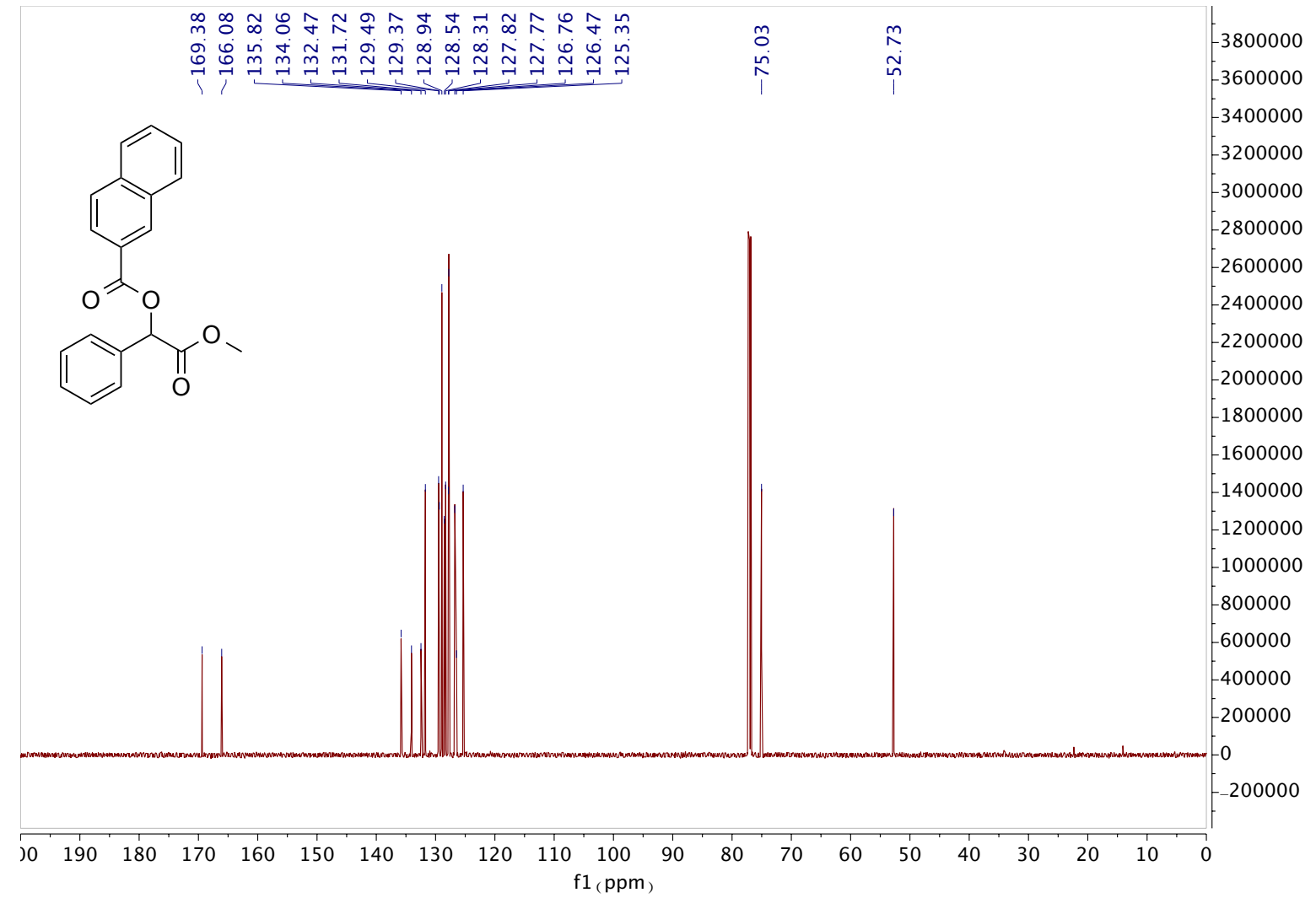


2-Methoxy-2-oxo-1-phenylethyl 1-naphthoate (7y)

${ }^{1} \mathbf{H}$ NMR $(600 \mathrm{MHz}$, Chloroform- $d$ )

Я̆ 乐

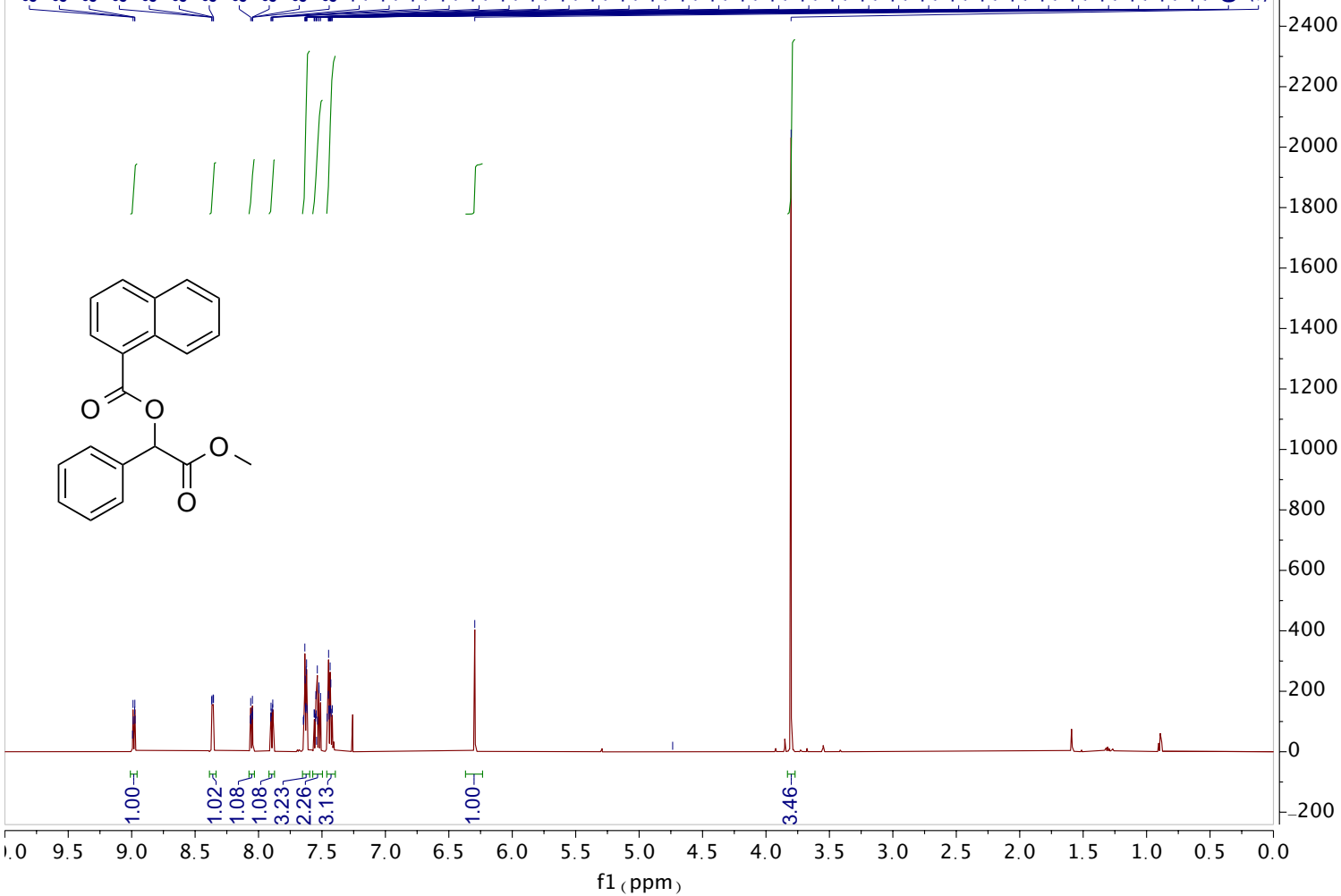

${ }^{13}$ C NMR (151 MHz, Chloroform- $d$ )

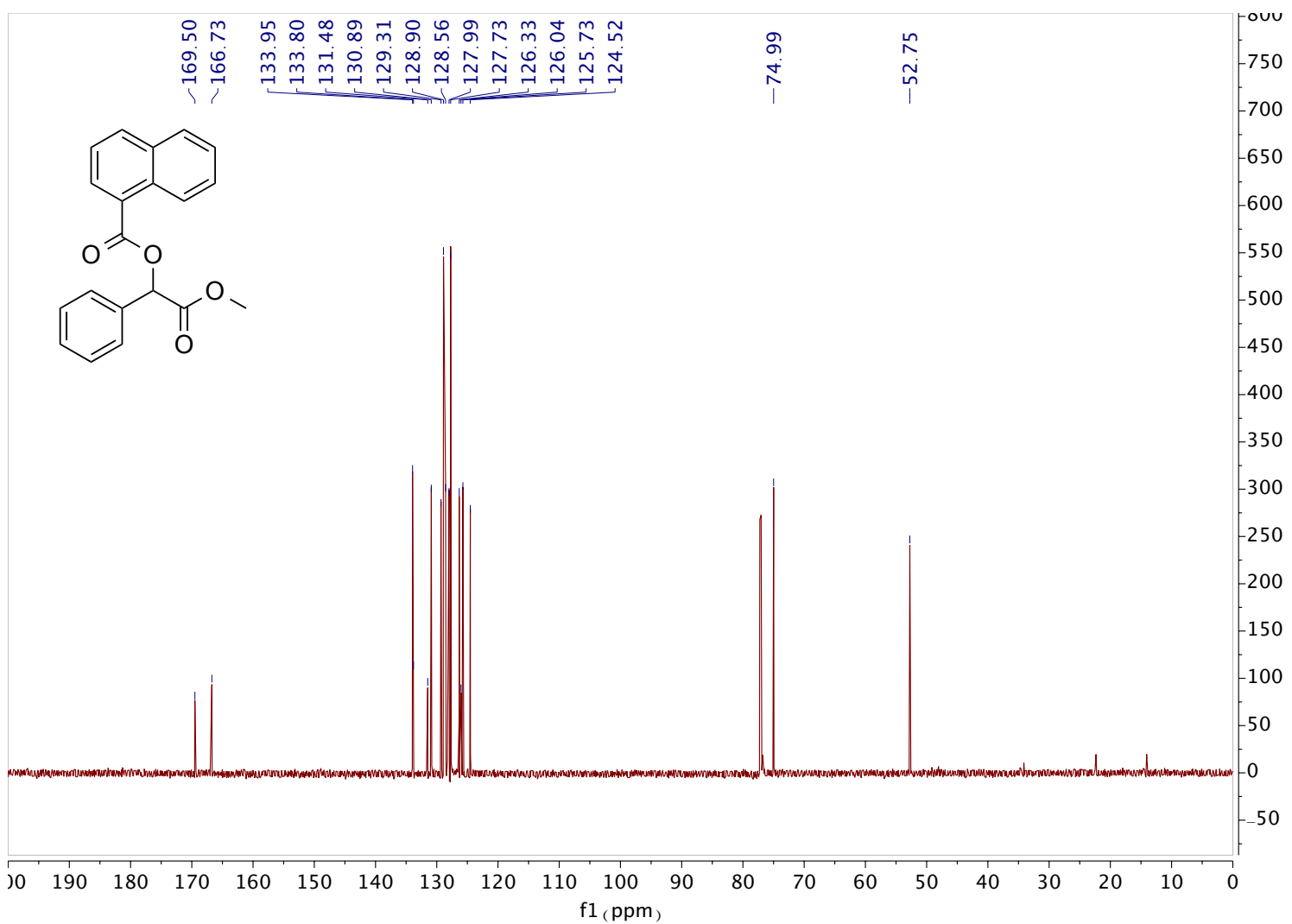


${ }^{1}$ H NMR (600 MHz, Chloroform- $d$ )

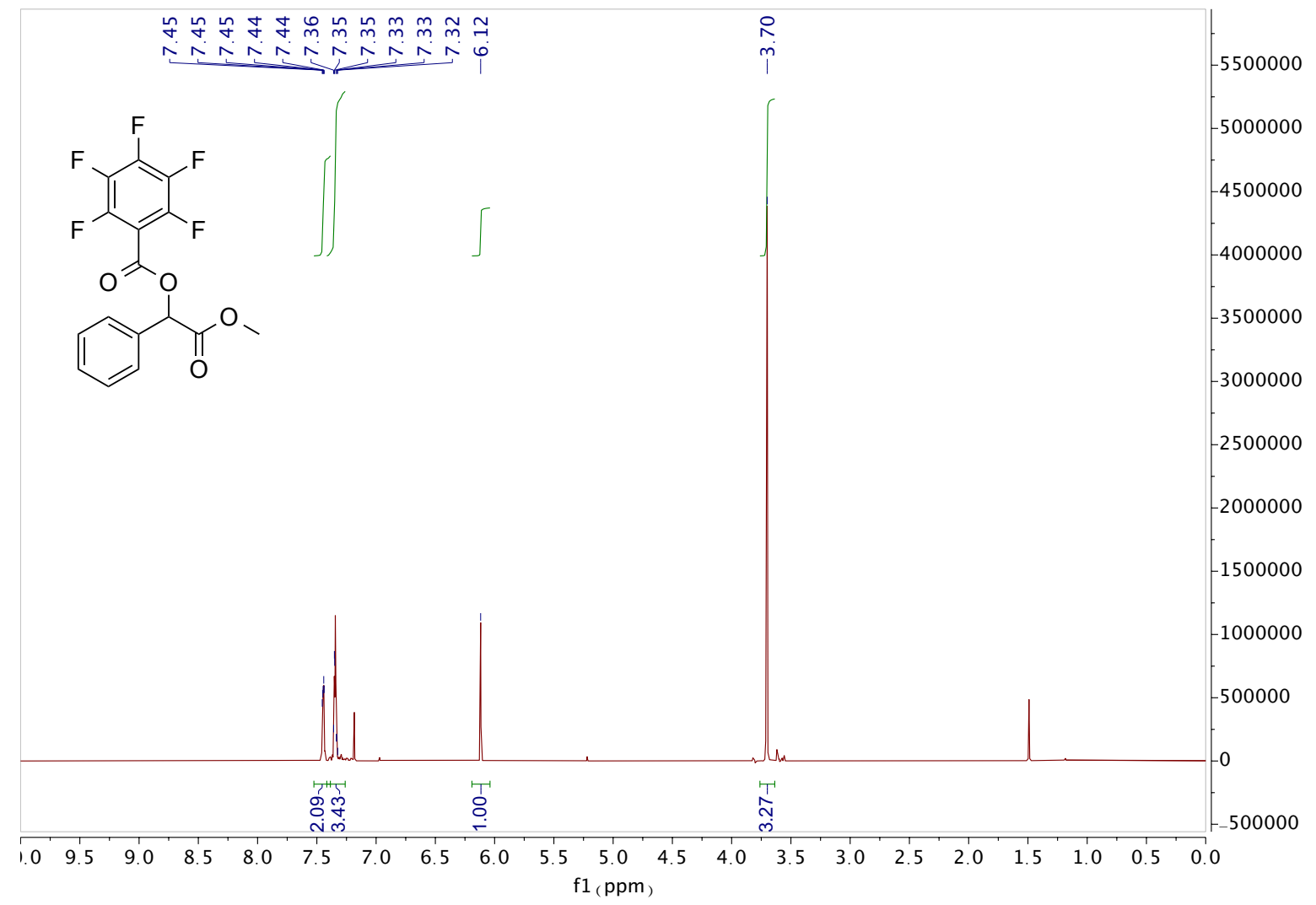

${ }^{13}$ C NMR (151 MHz, Chloroform- $d$ )

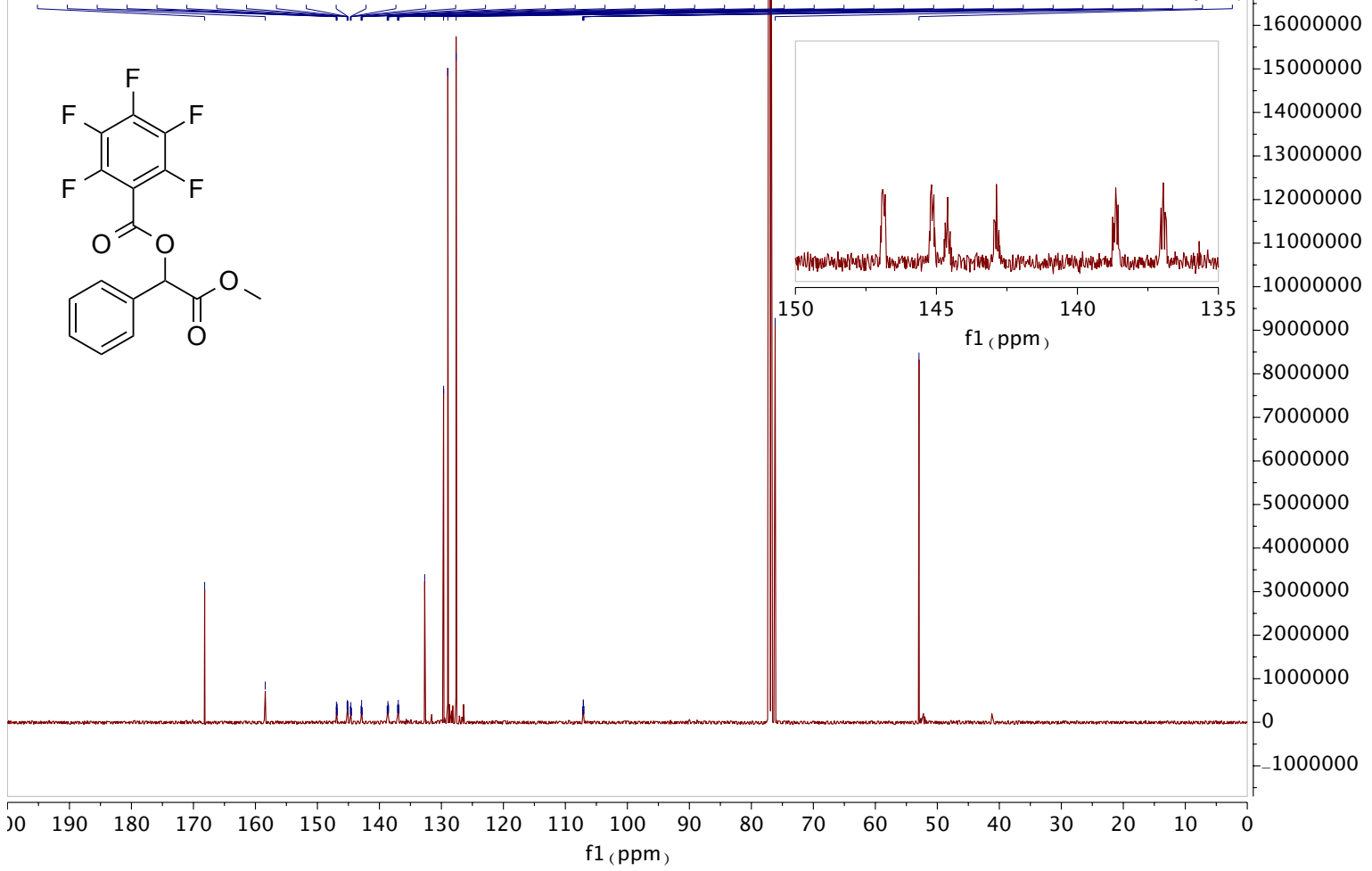


${ }^{19}$ F NMR (565 MHz, Chloroform-d)

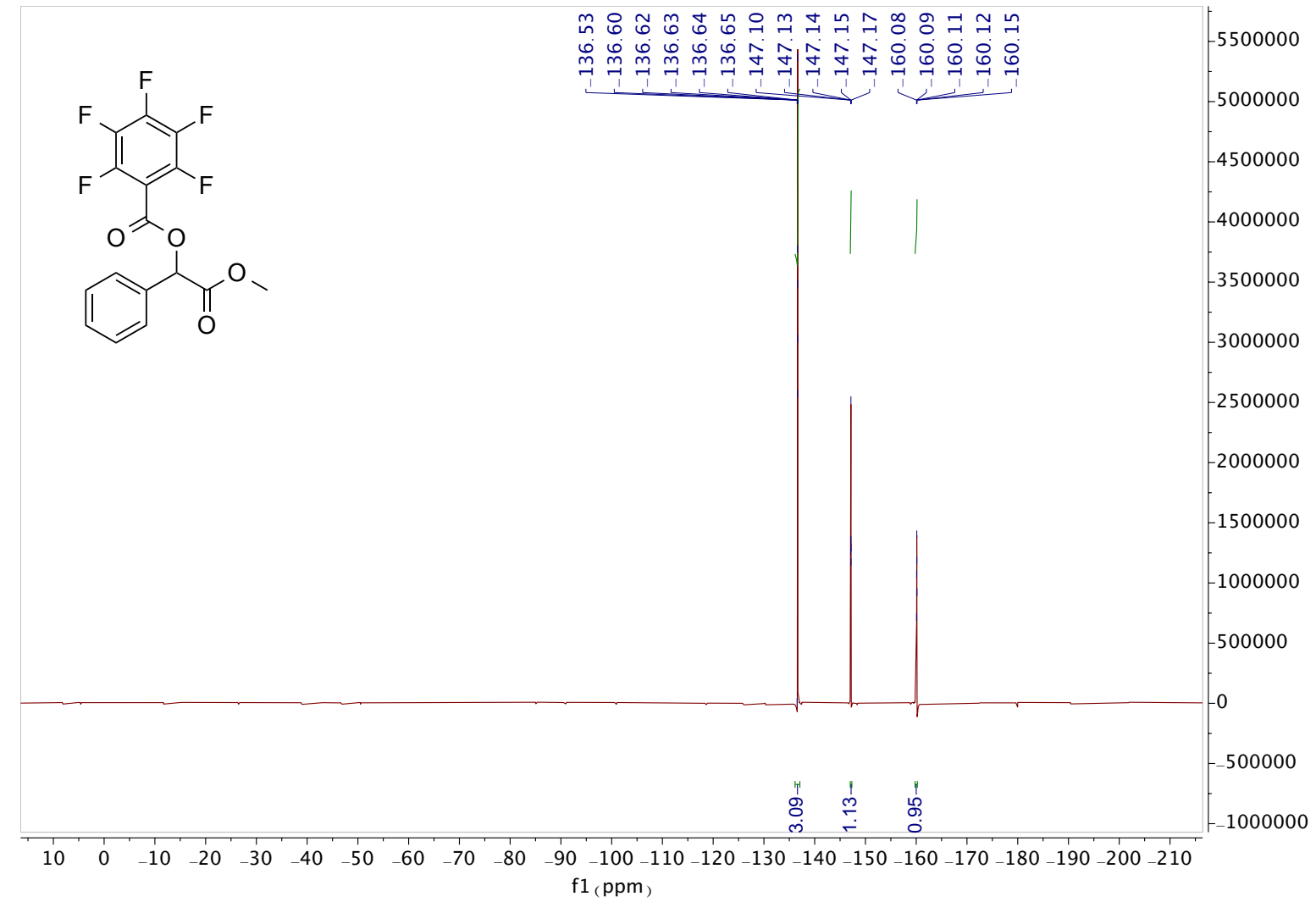


${ }^{1}$ H NMR (600 MHz, Chloroform- $d$ )

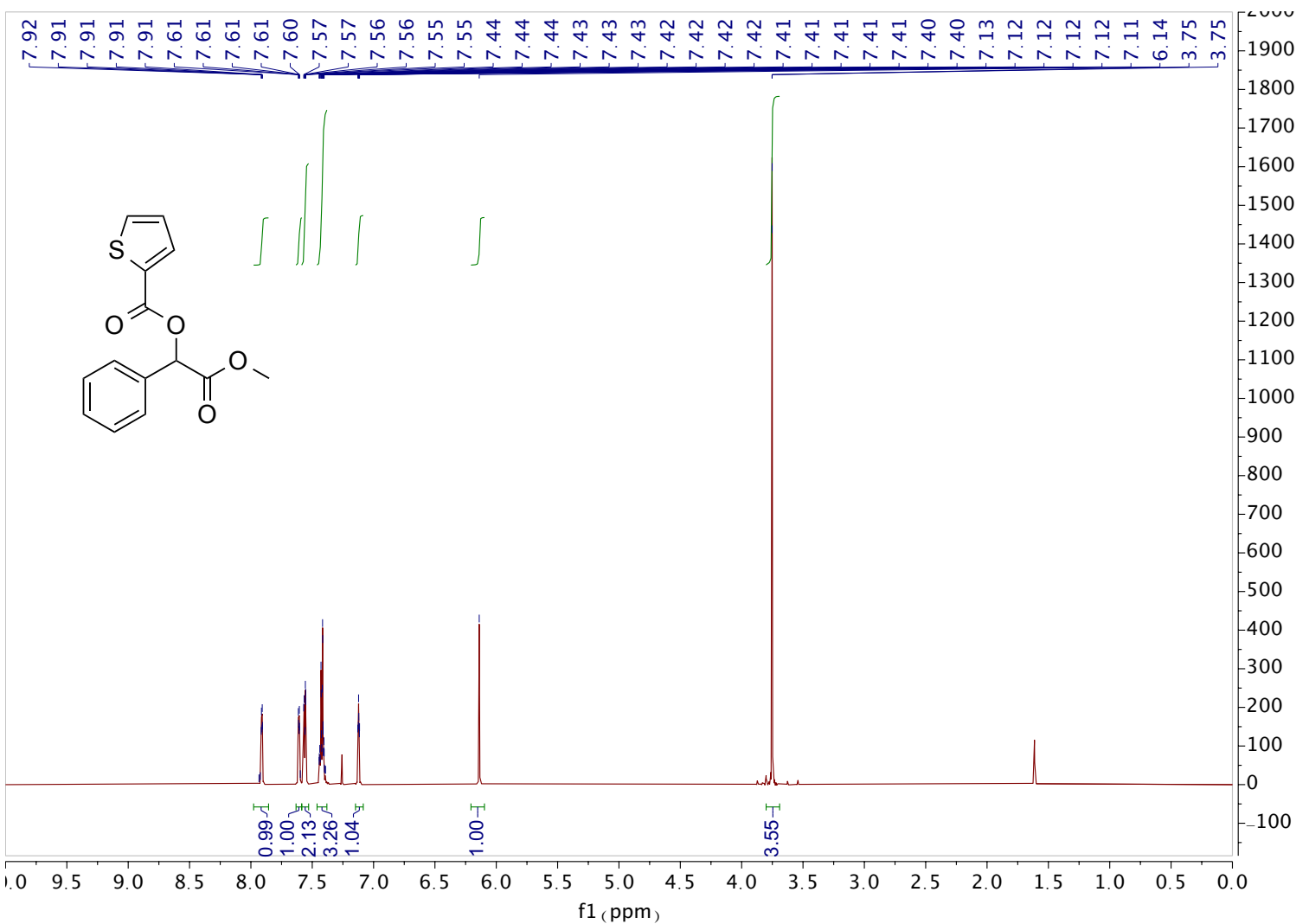

${ }^{13}$ C NMR (151 MHz, Chloroform- $d$ )

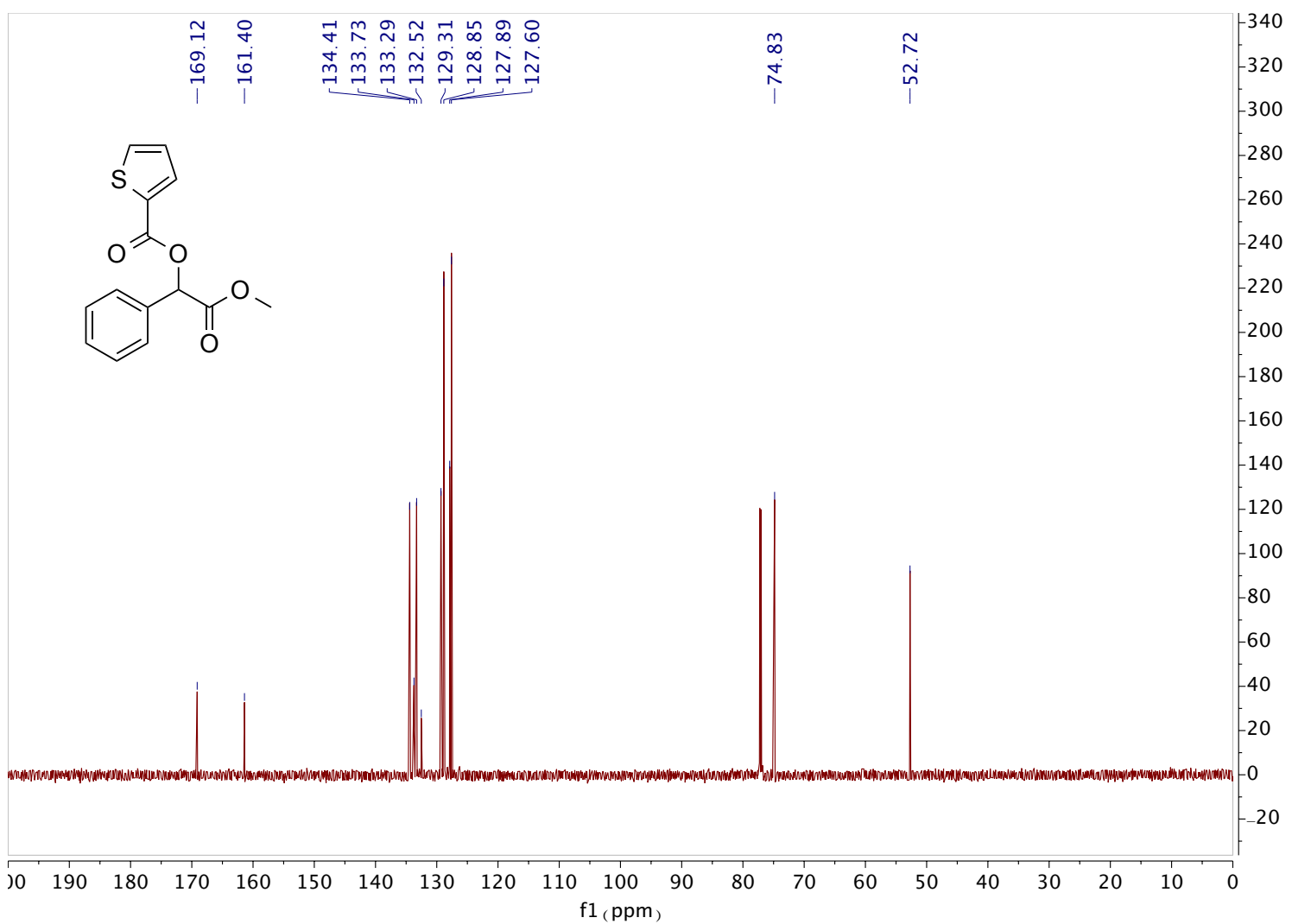




\section{2,2,2-Trifluoro-1-phenylethyl benzoate (8)}

${ }^{1}$ H NMR (600 MHz, Chloroform- $d$ )

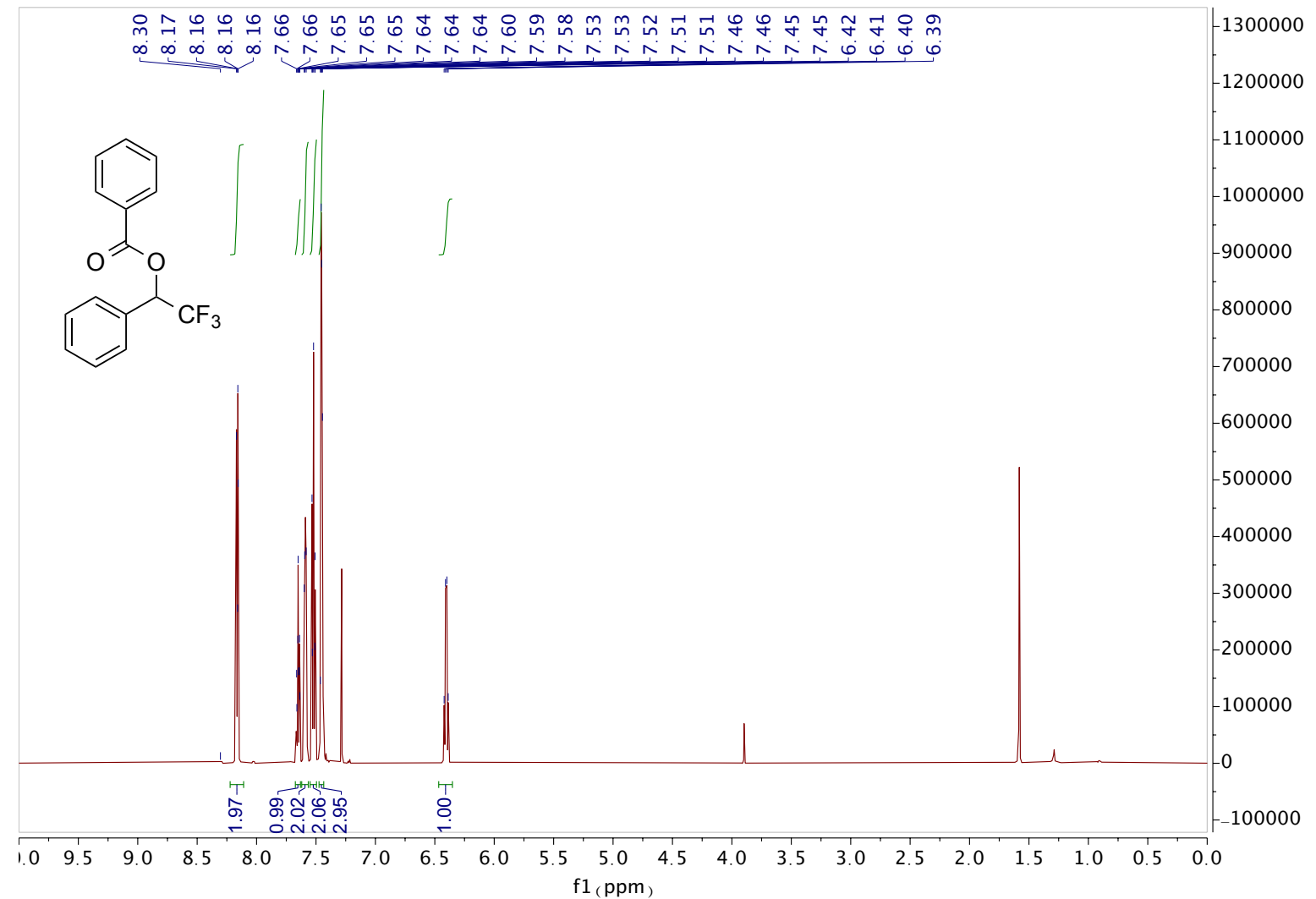

${ }^{13}$ C NMR (151 MHz, Chloroform- $d$ )

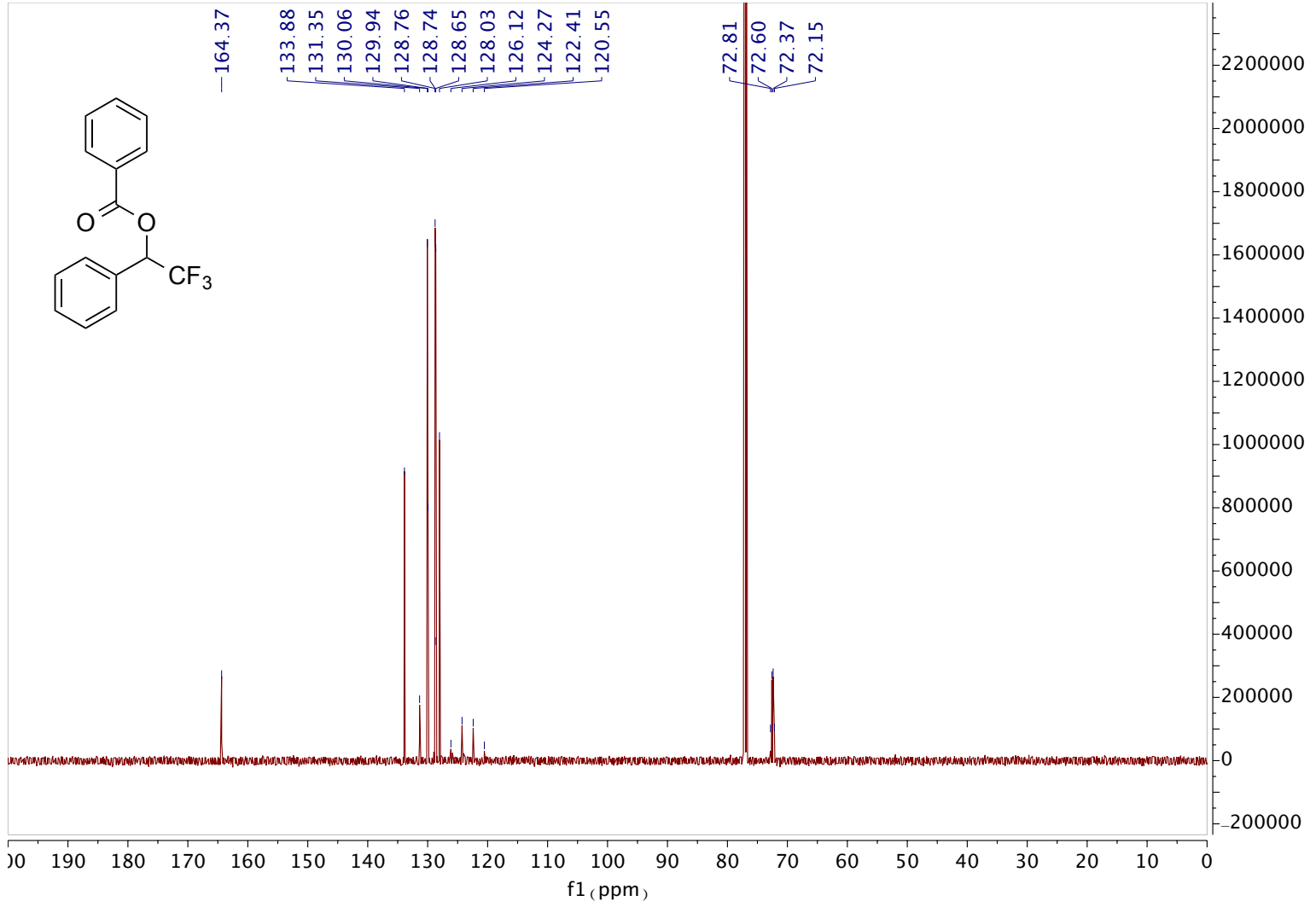


${ }^{19}$ F NMR (565 MHz, Chloroform-d)

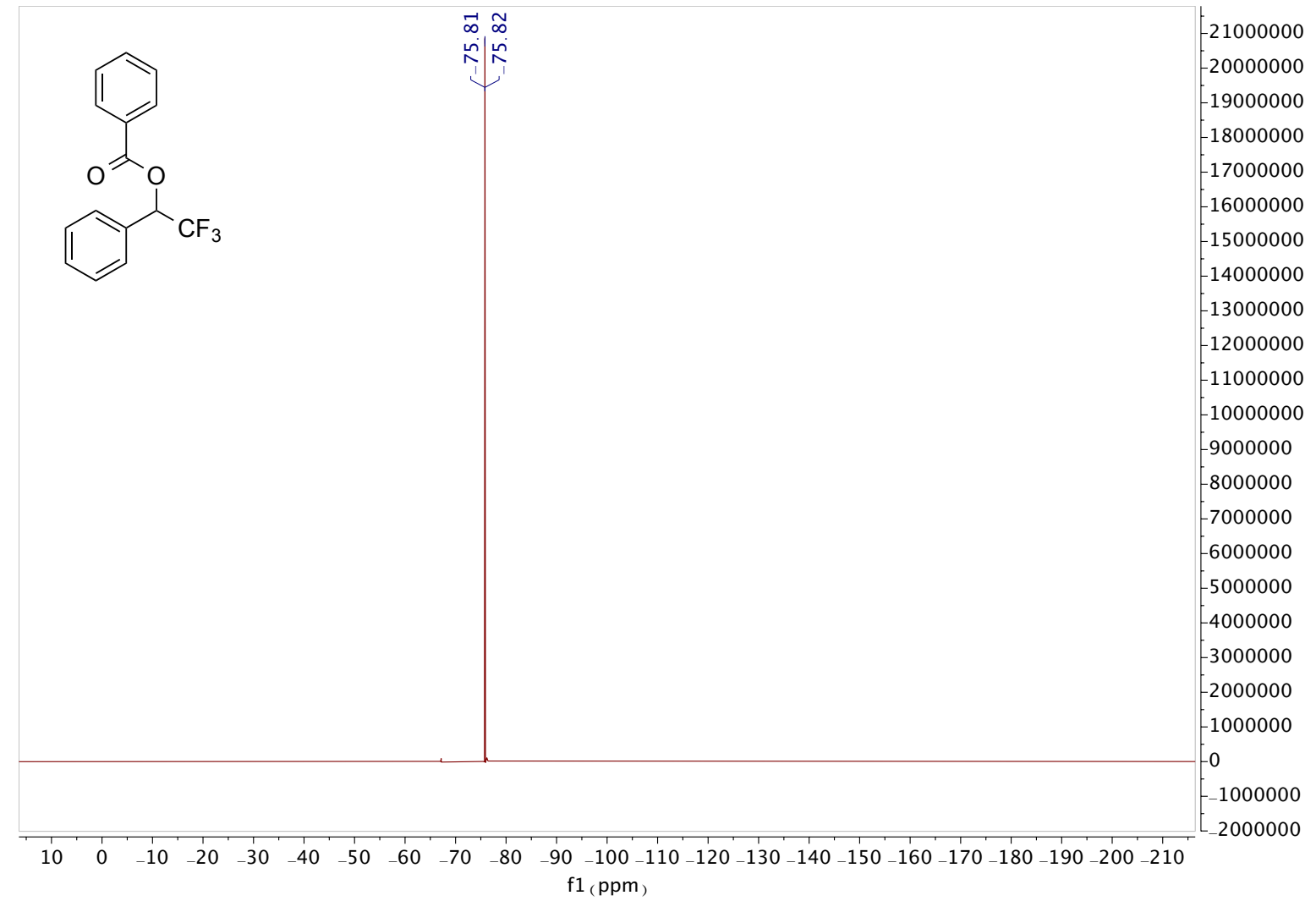




\section{Benzhydryl benzoate (9)}

\section{${ }^{1}$ H NMR (400 MHz, Chloroform- $d$ )}

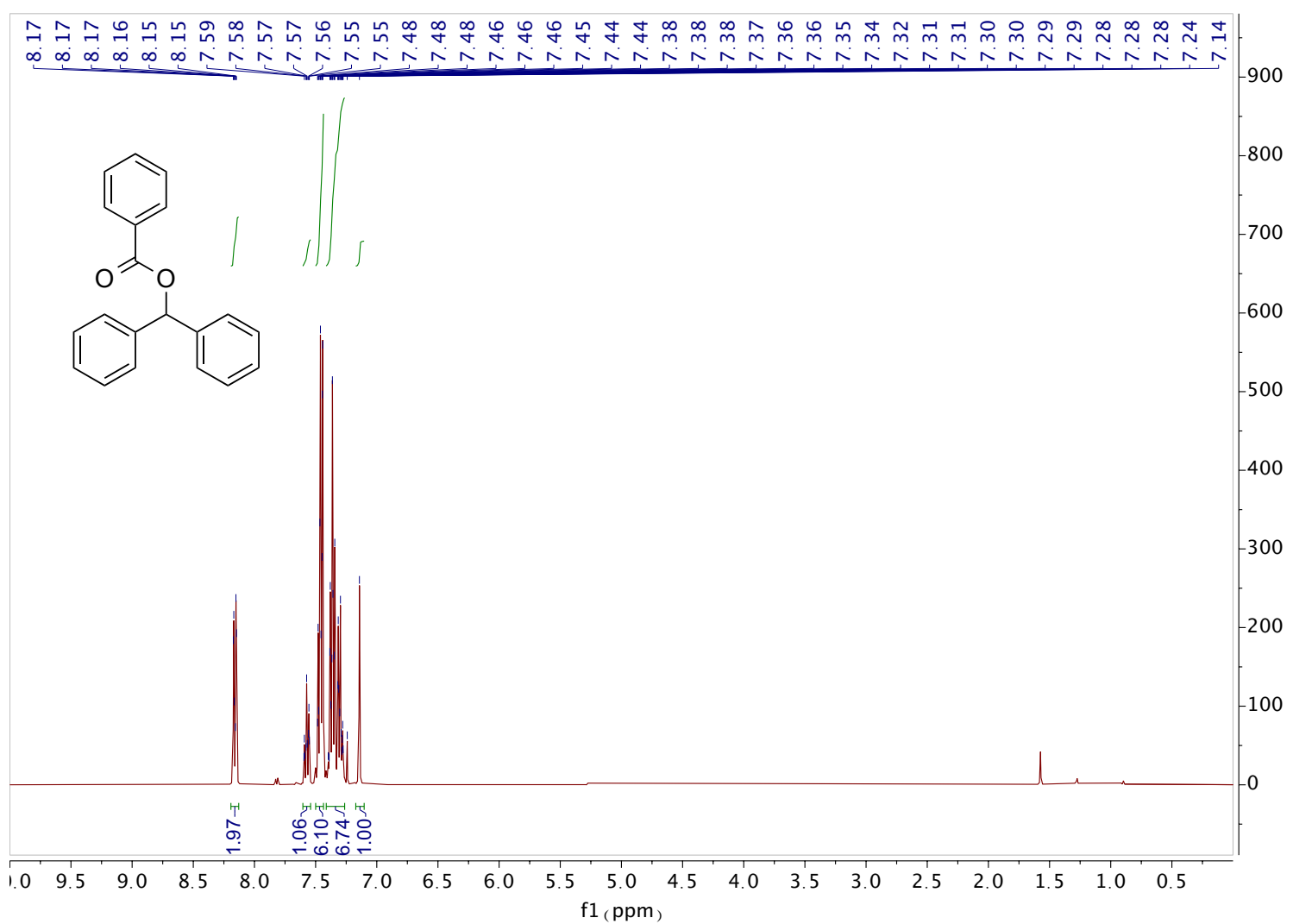

${ }^{13}$ C NMR (101 MHz, Chloroform- $d$ )

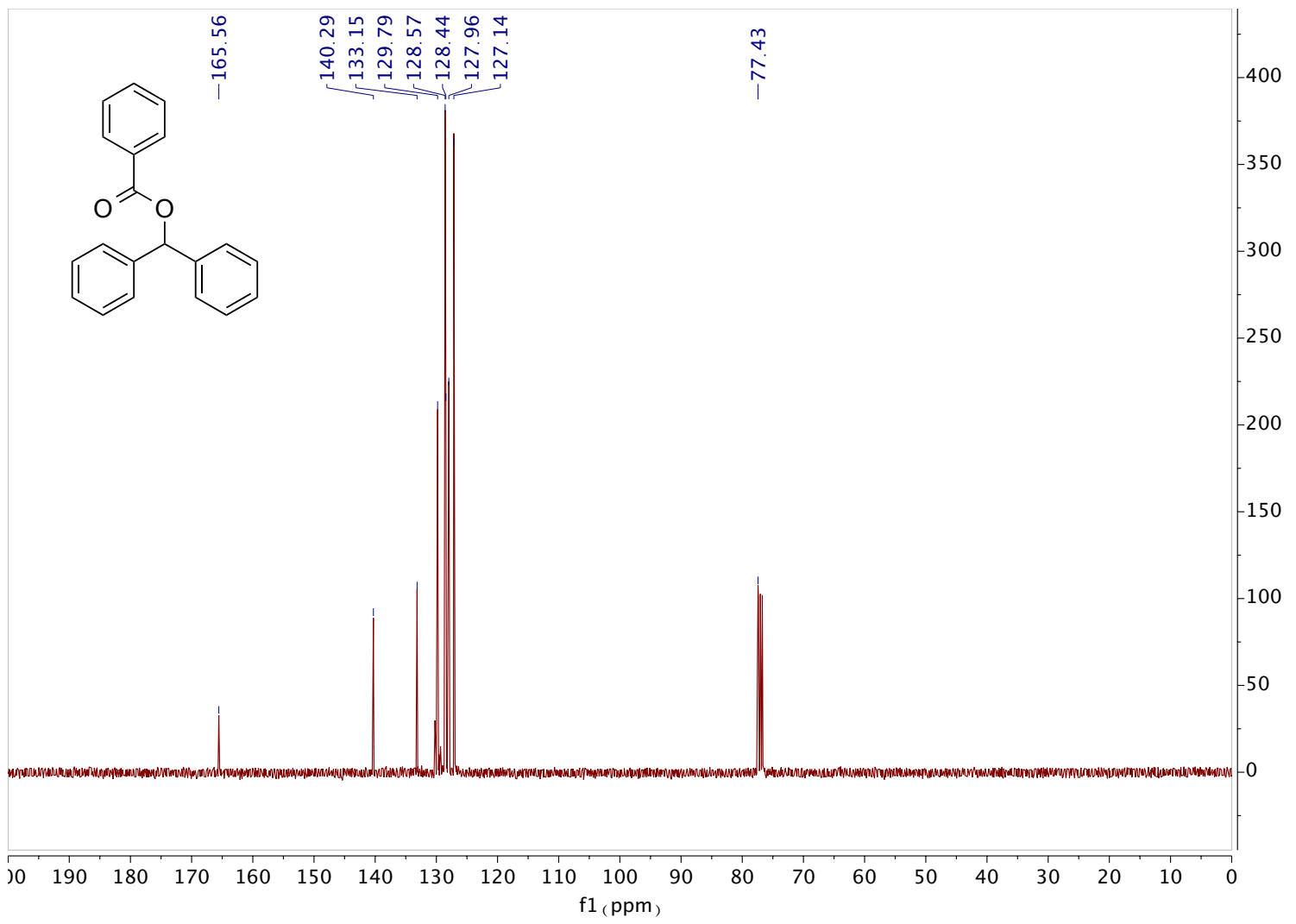


2-Ethoxy-2-oxoethyl benzoate (10)

${ }^{1}$ H NMR (600 MHz, Chloroform- $d$ )

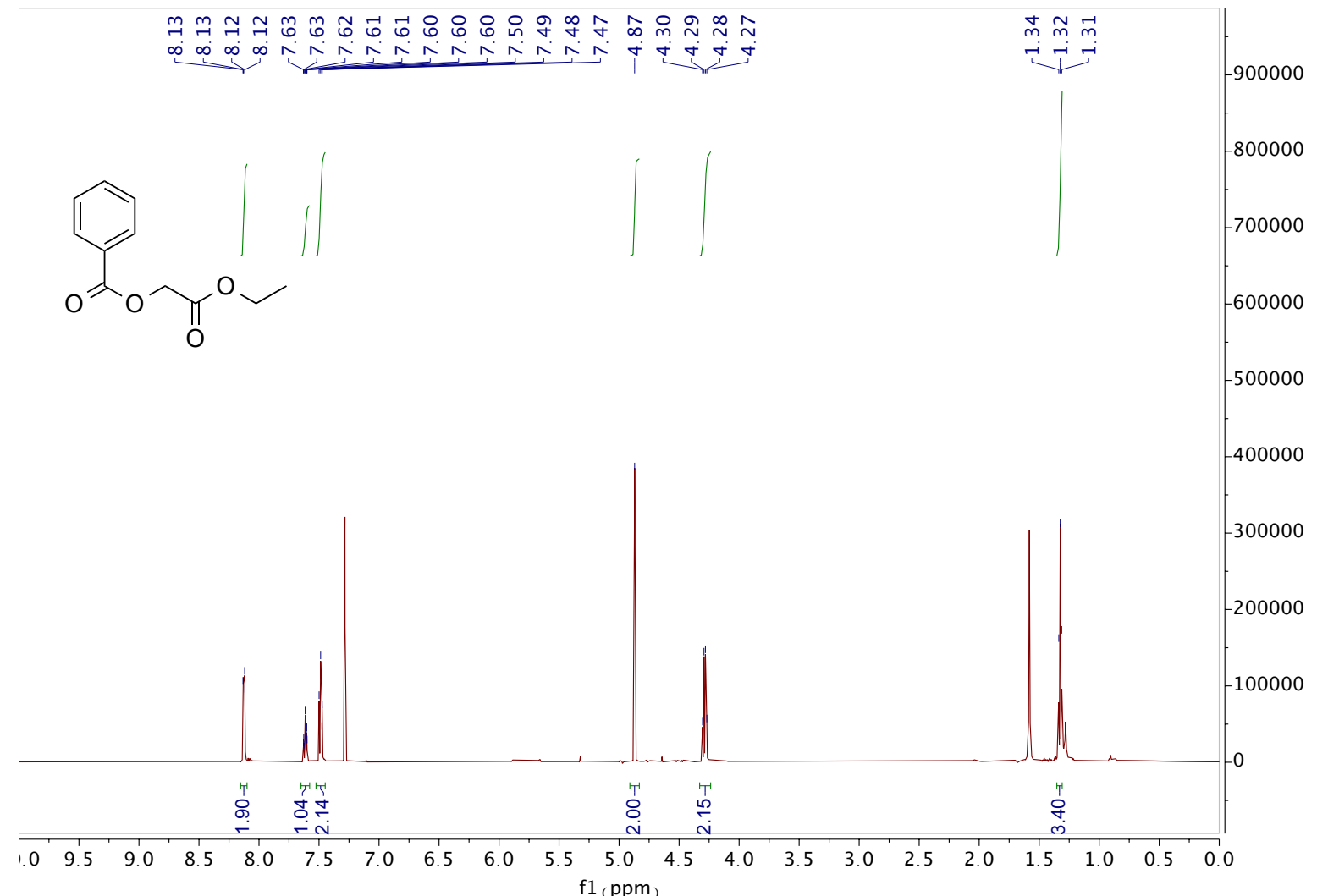

${ }^{13}$ C NMR (151 MHz, Chloroform- $d$ )

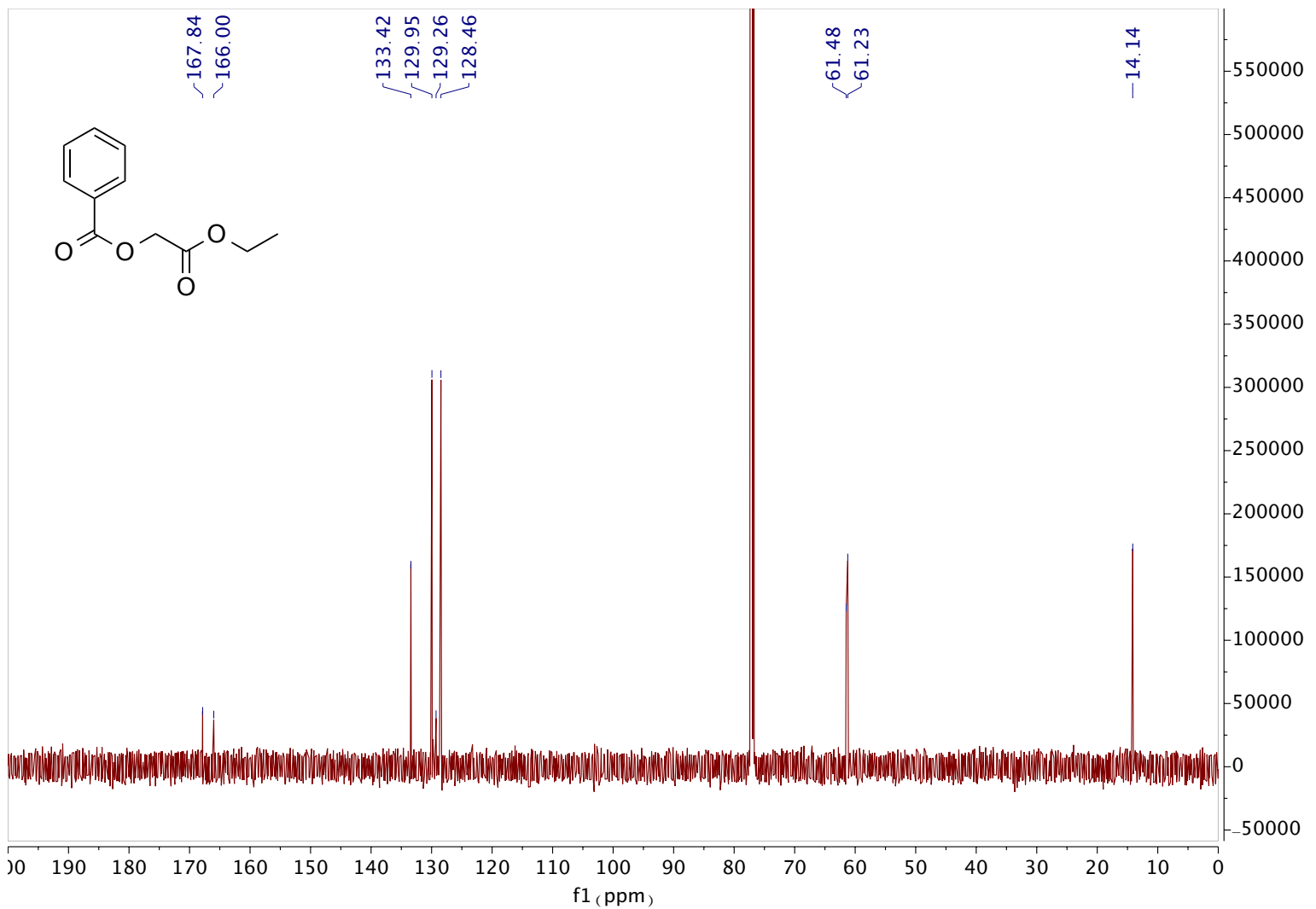




\section{${ }^{1}$ H NMR (600 MHz, Chloroform- $d$ )}

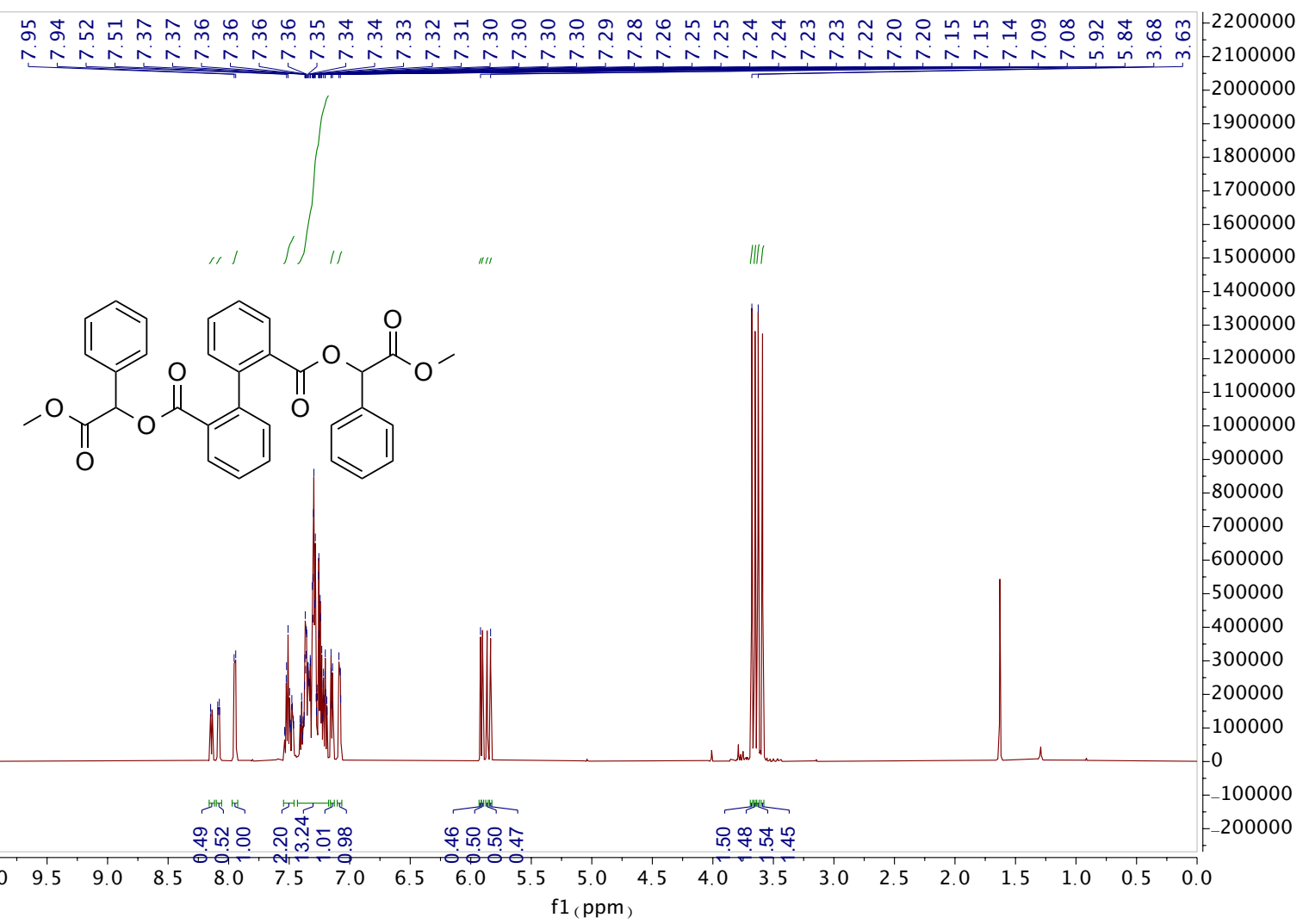

${ }^{13}$ C NMR (151 MHz, Chloroform- $d$ )

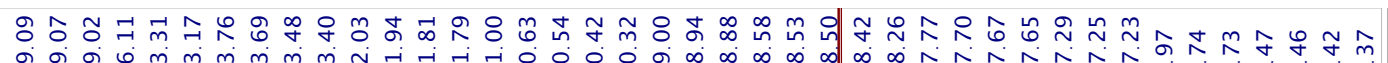

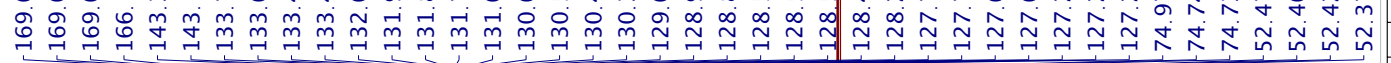

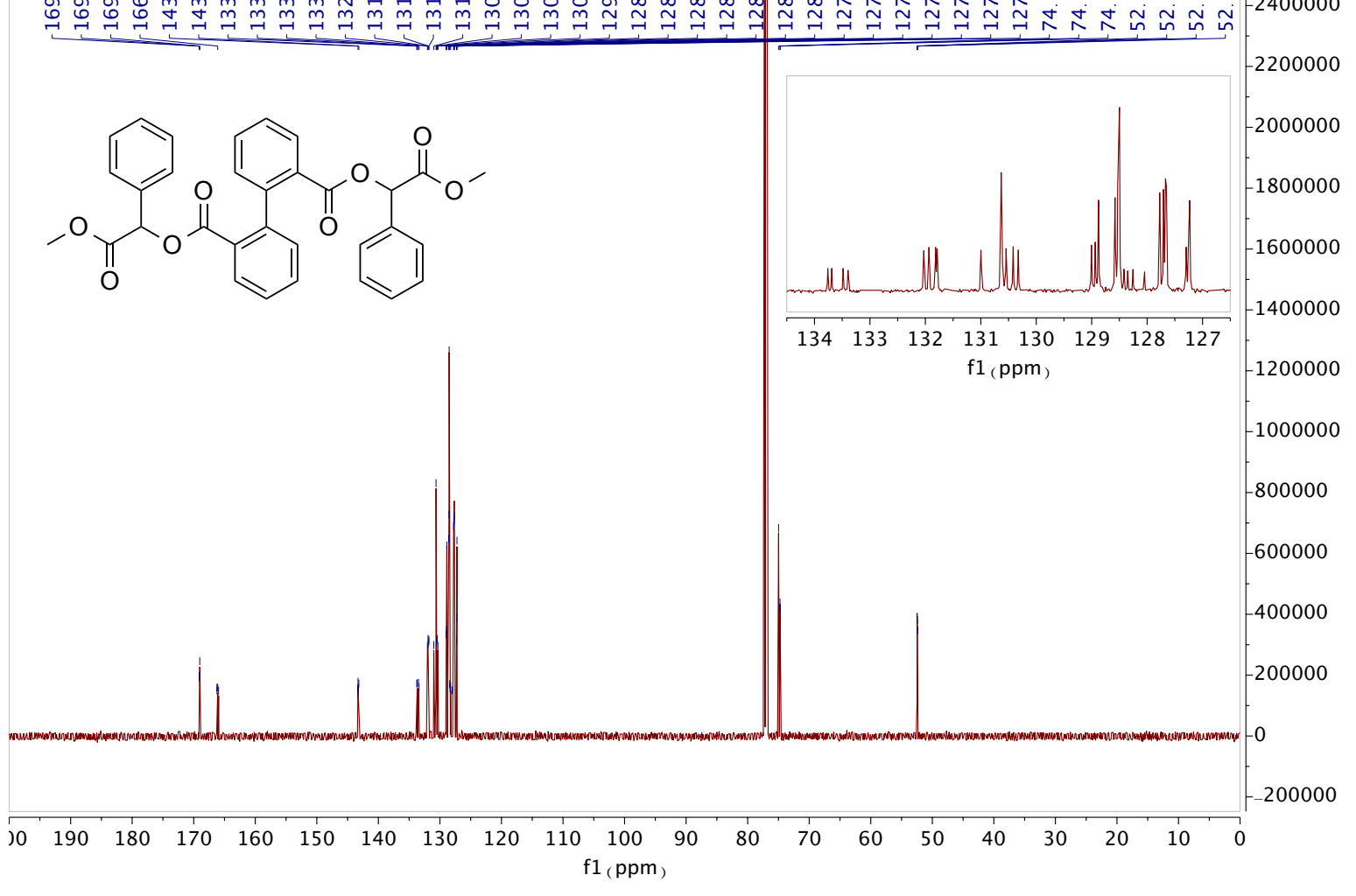


Methyl 2-acetoxy-2-phenylacetate (12a)

${ }^{1}$ H NMR (400 MHz, Chloroform- $d$ )

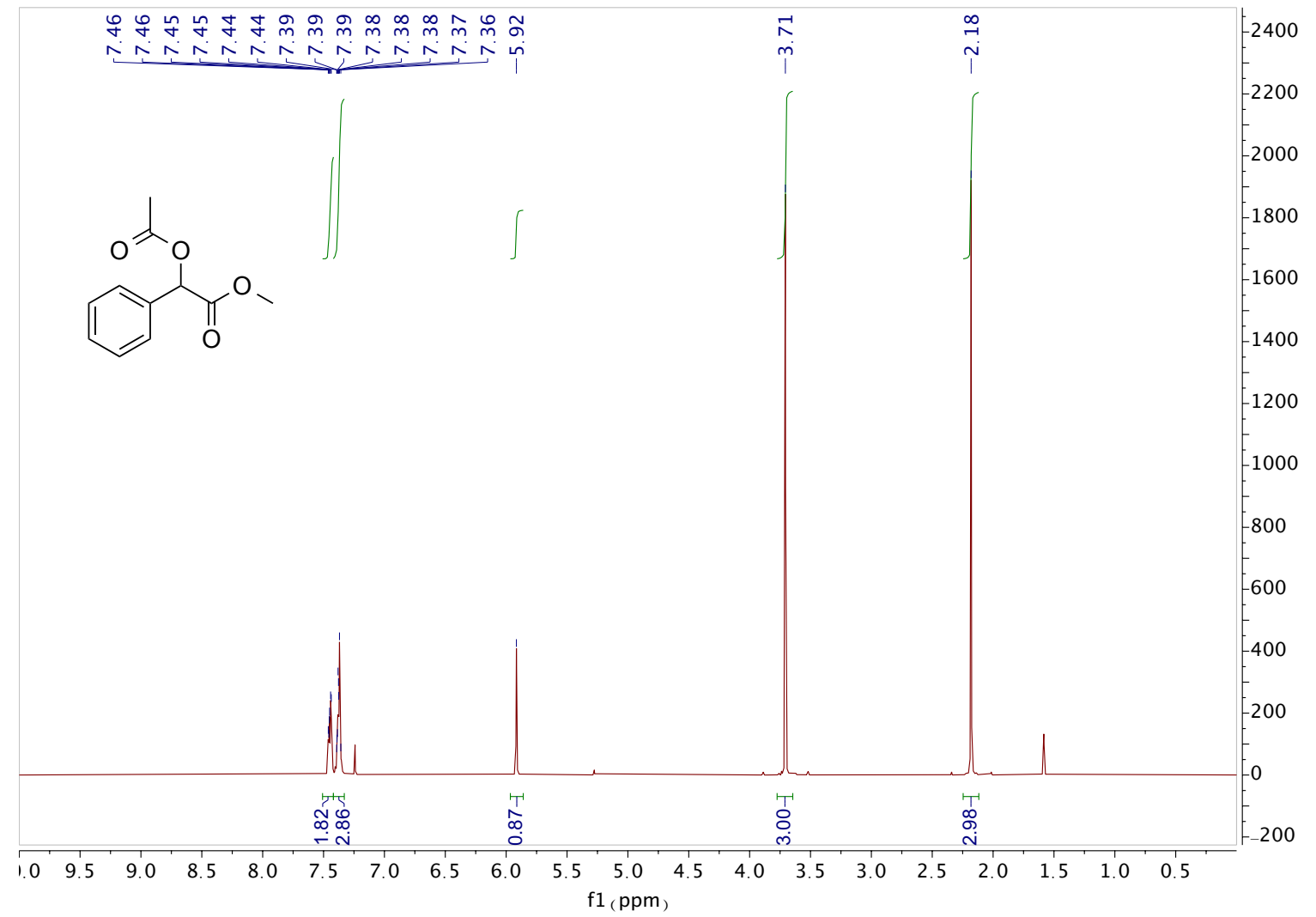

${ }^{13}$ C NMR (101 MHz, Chloroform- $d$ )

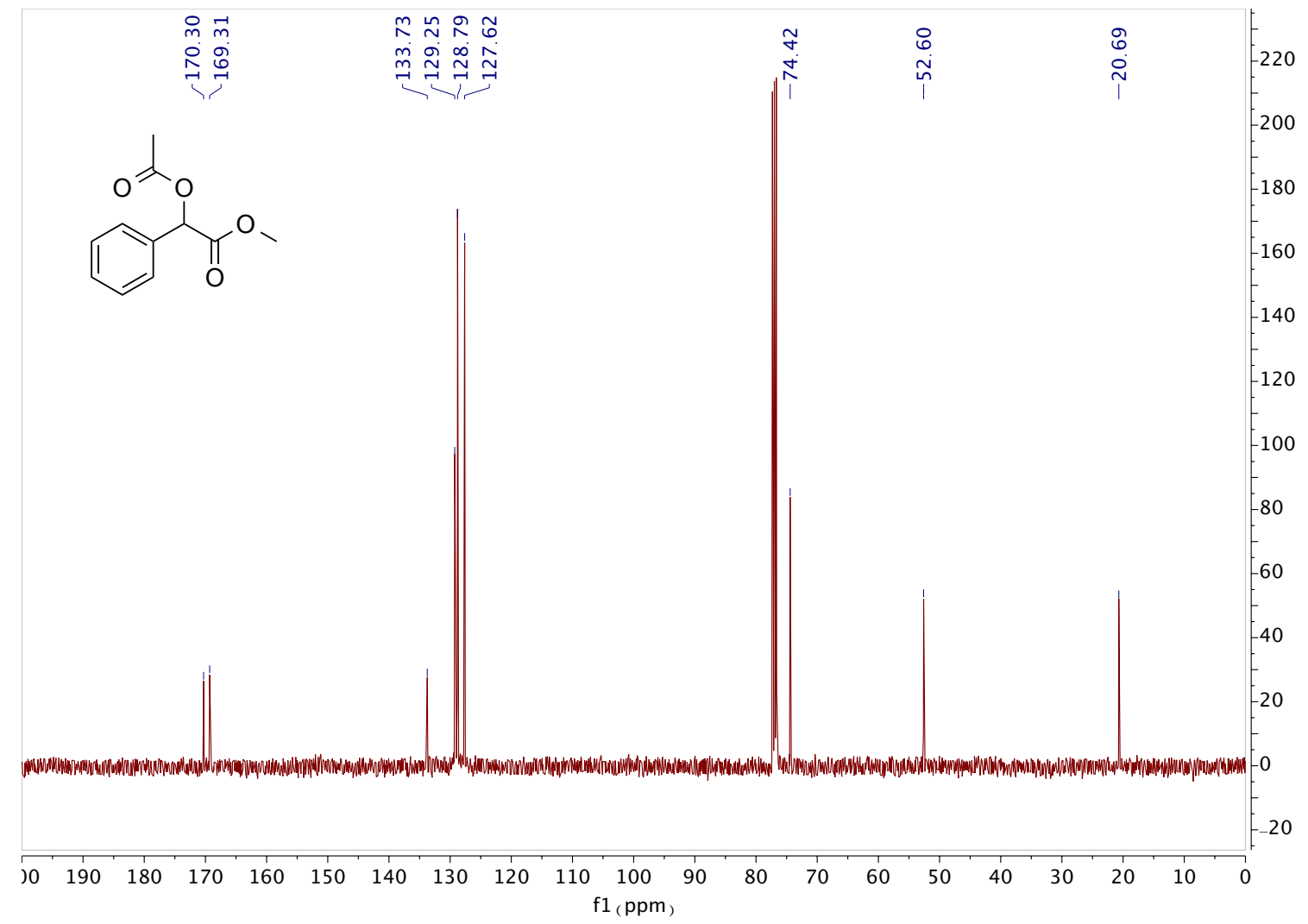


2-Methoxy-2-oxo-1-phenylethyl propionate (12b)

${ }^{1}$ H NMR (400 MHz, Chloroform- $d$ )

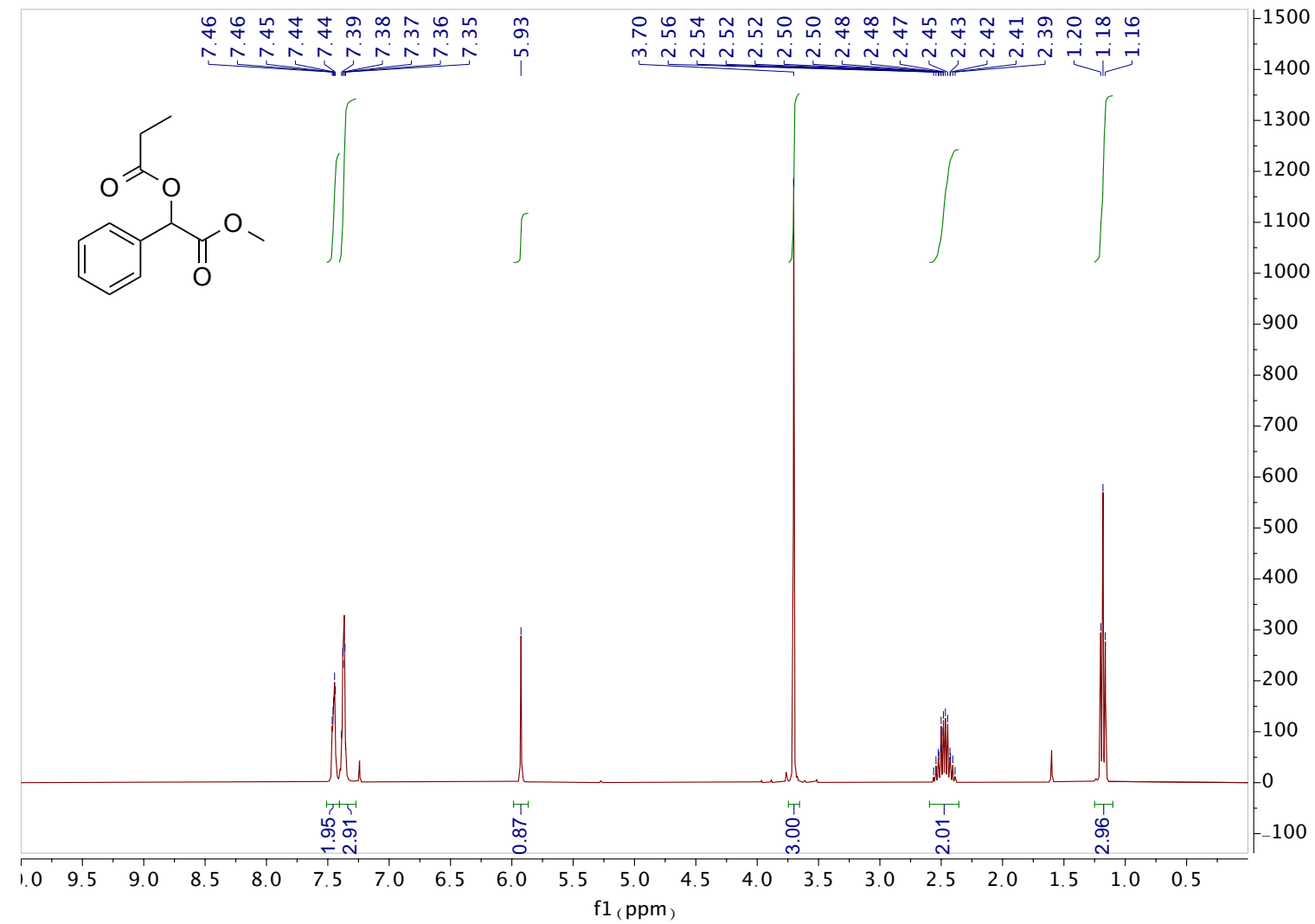

${ }^{13}$ C NMR (101 MHz, Chloroform- $d$ )

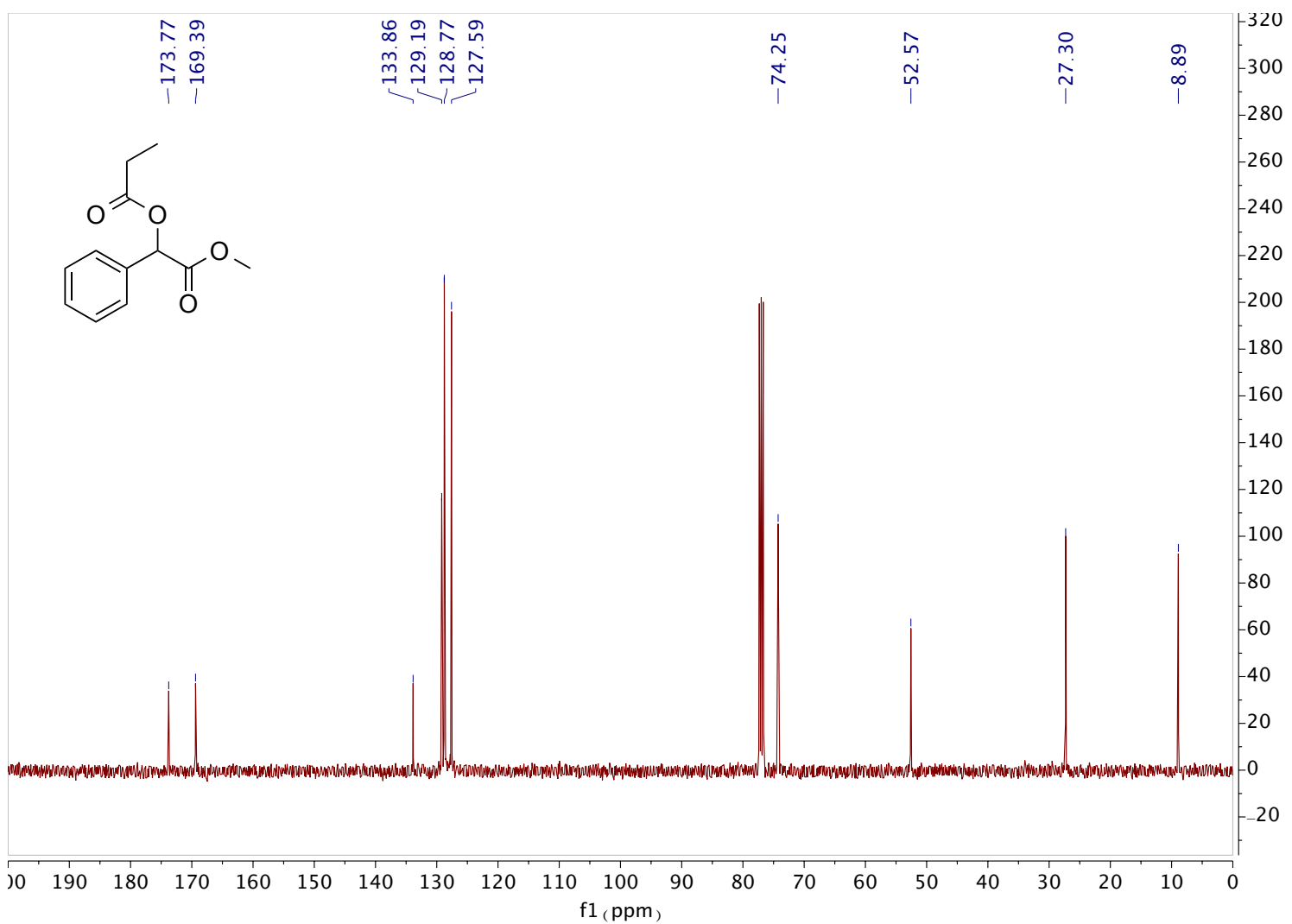


${ }^{1}$ H NMR (400 MHz, Chloroform- $d$ )

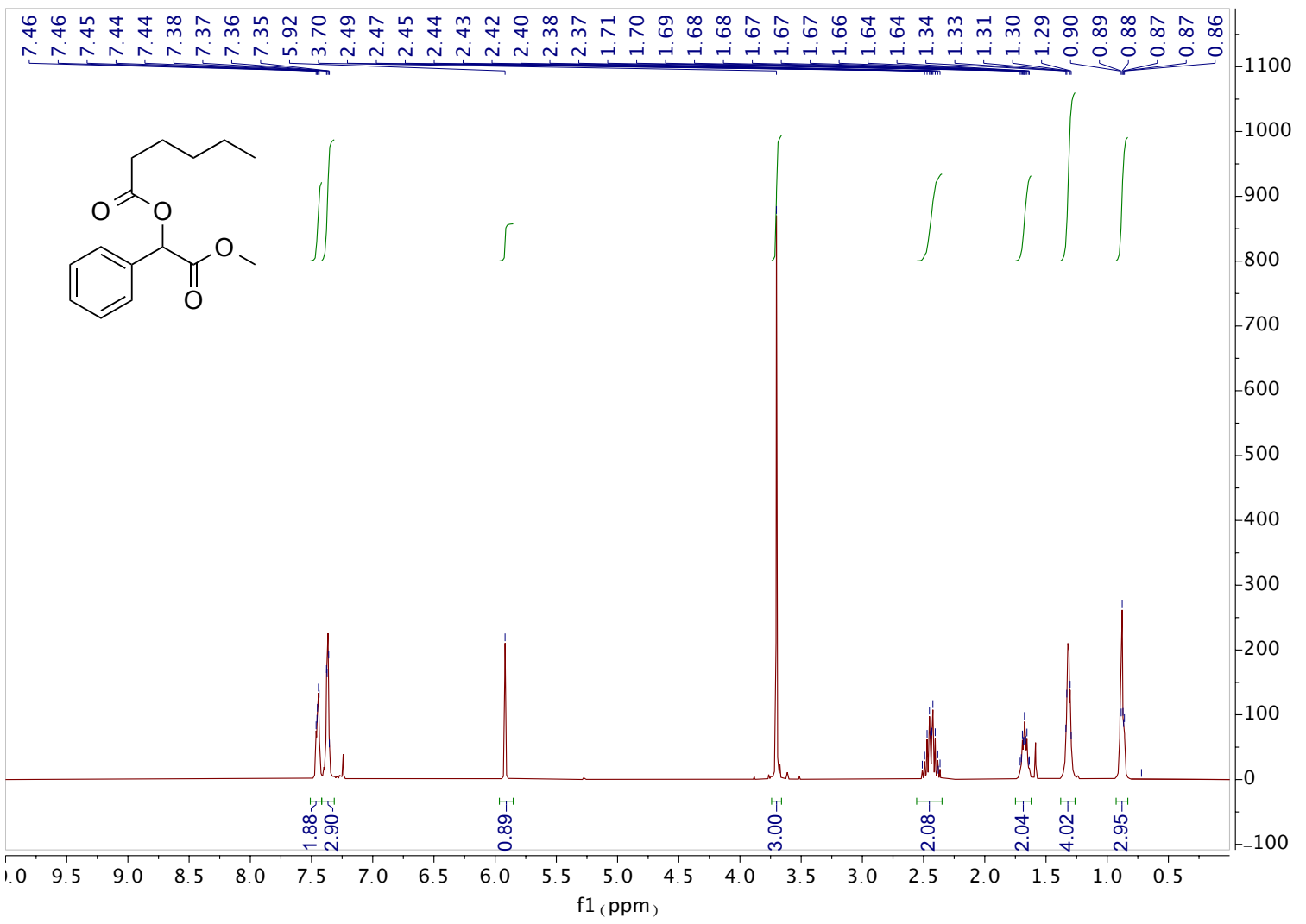

${ }^{13}$ C NMR (101 MHz, Chloroform- $d$ )

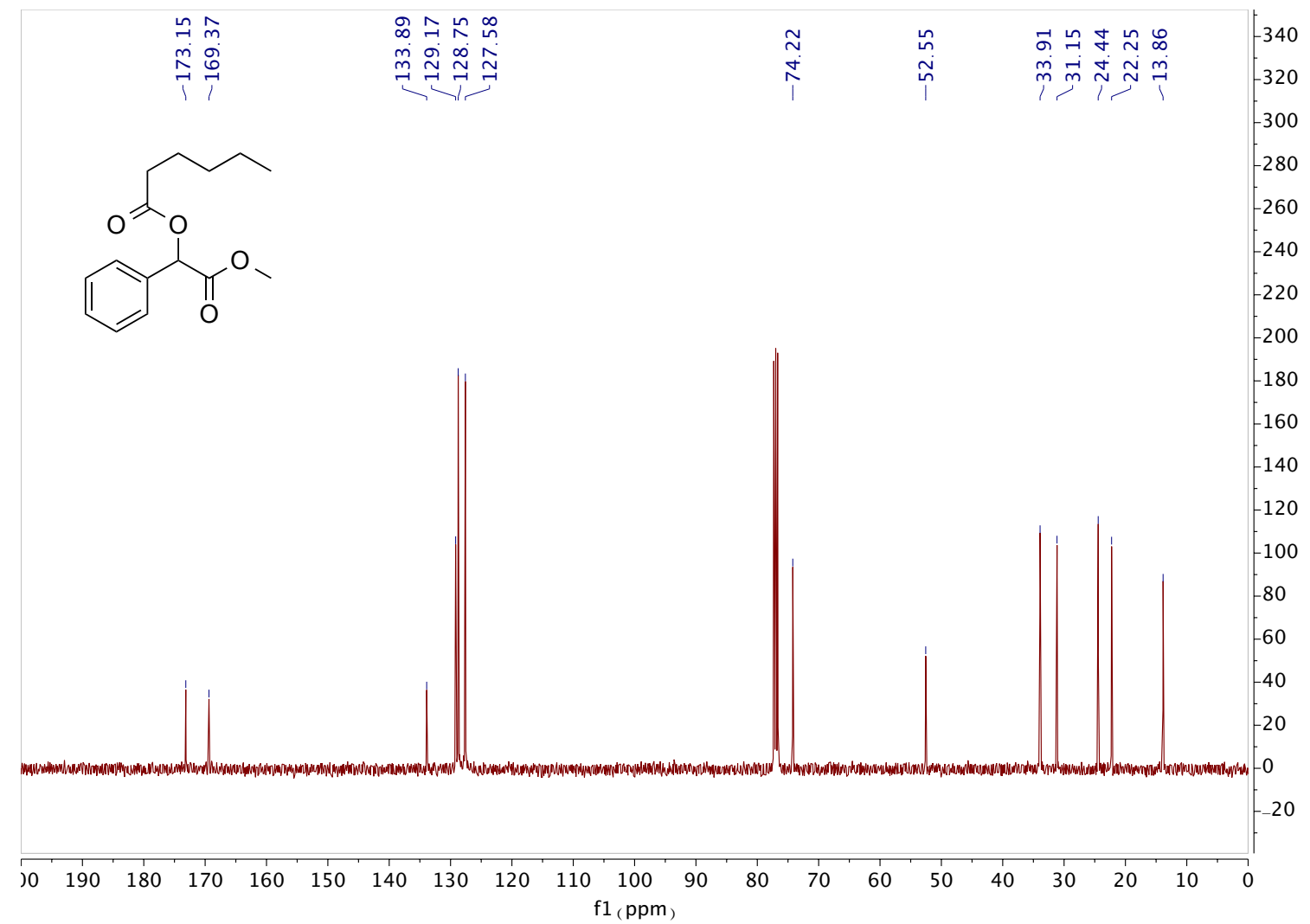


${ }^{1}$ H NMR (400 MHz, Chloroform- $d$ )

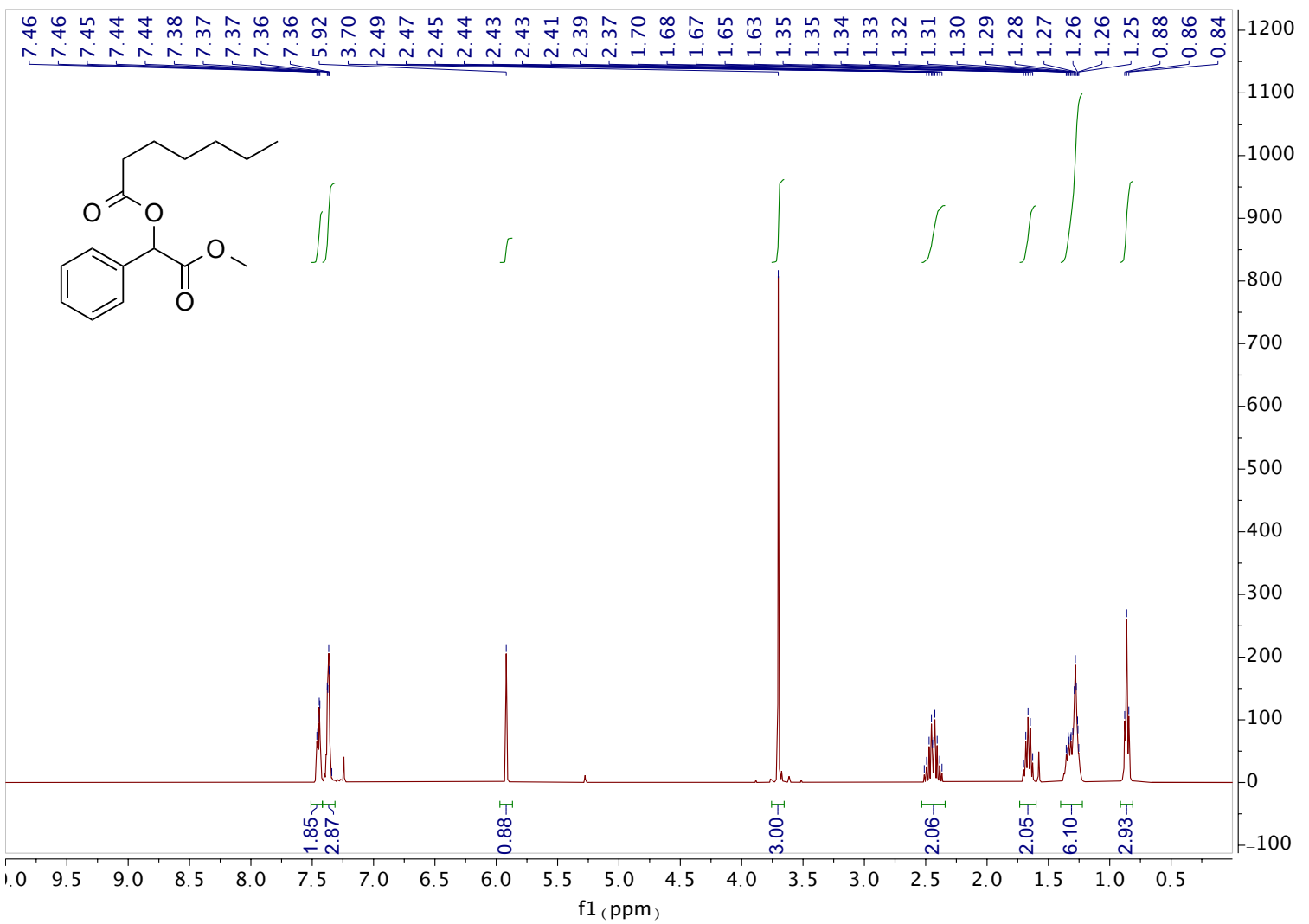

${ }^{13}$ C NMR (101 MHz, Chloroform- $d$ )

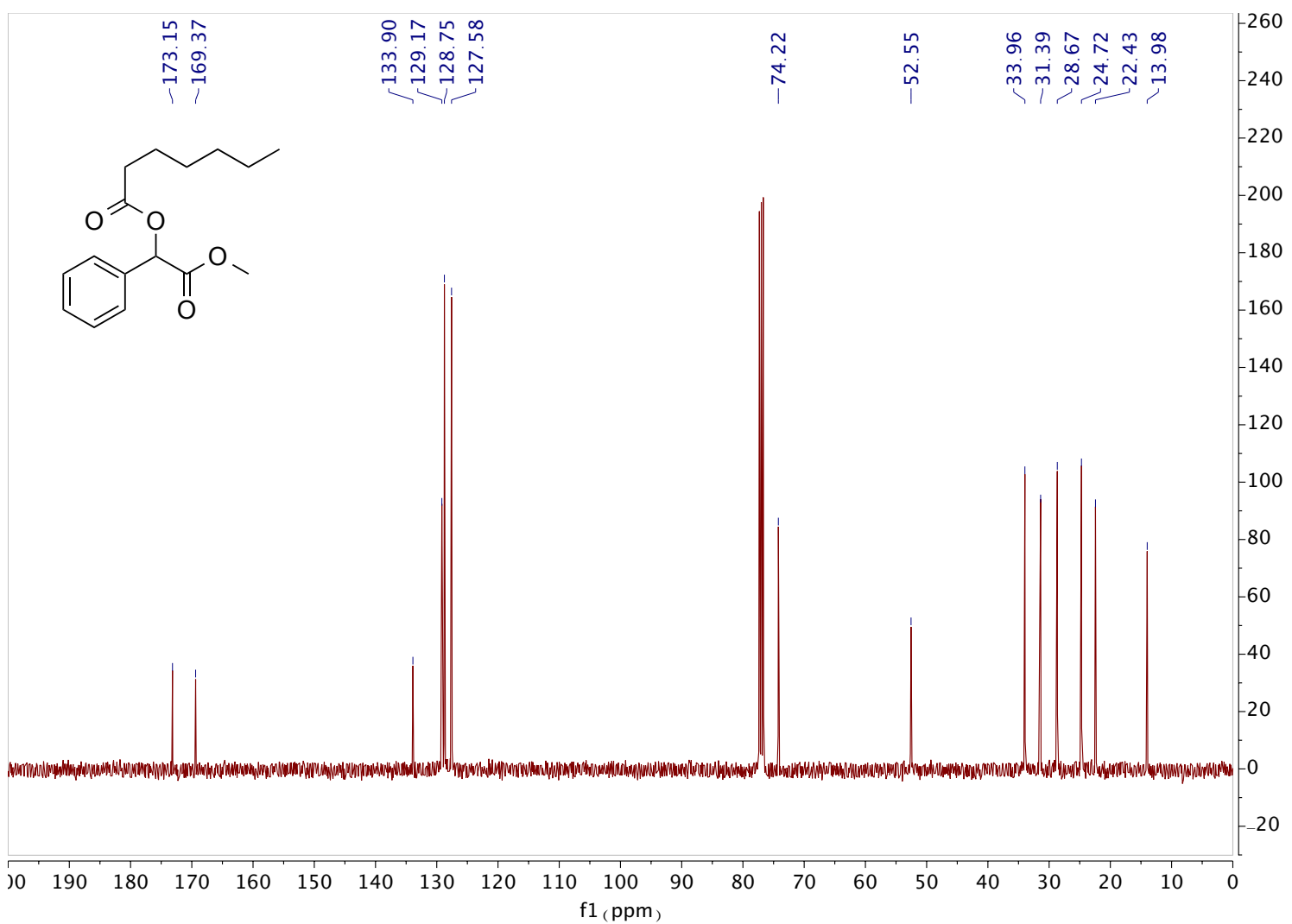


Methyl 2-phenyl-2-(2-phenylacetoxy)acetate (12e)

${ }^{1}$ H NMR (400 MHz, Chloroform- $d$ )

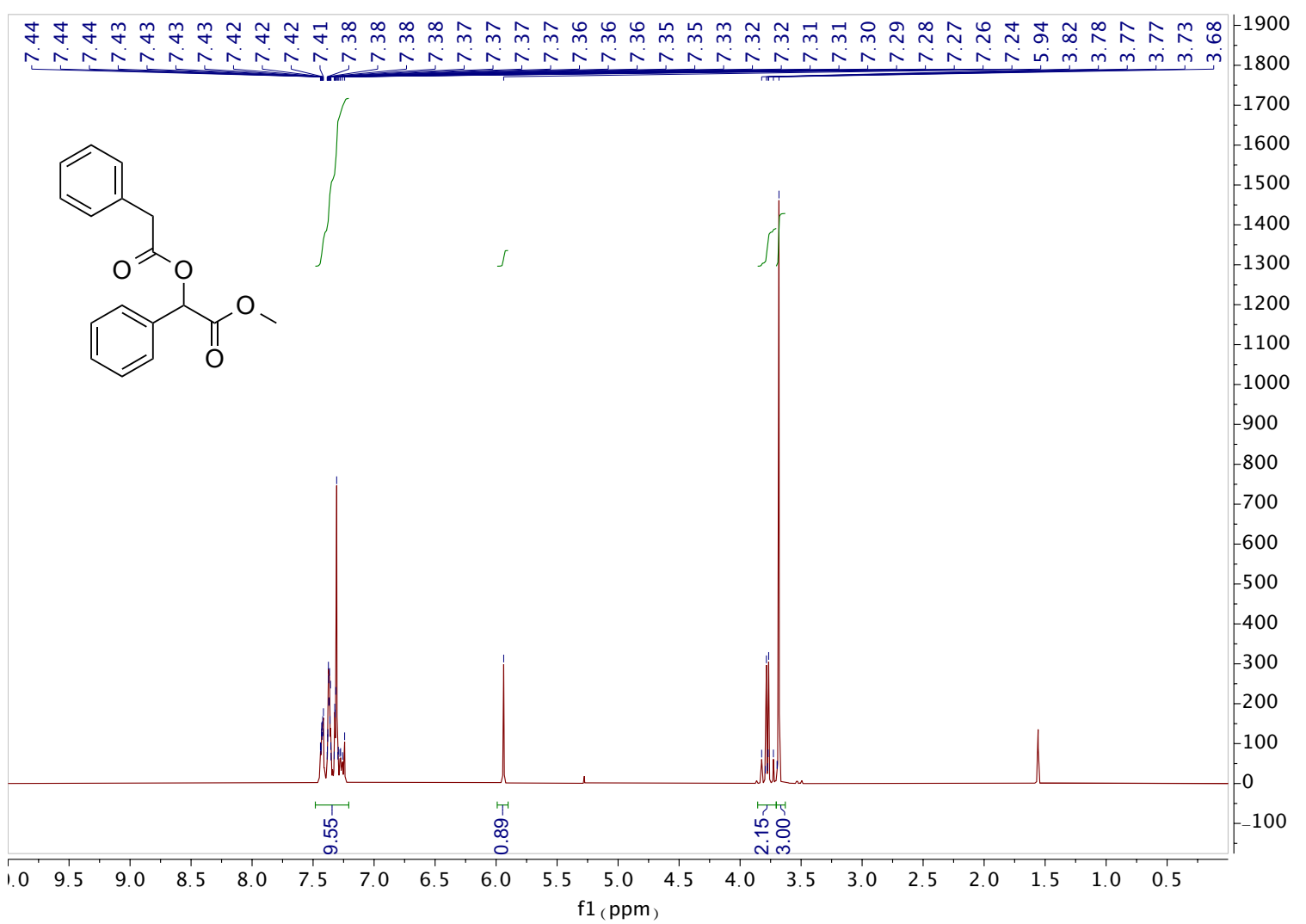

${ }^{13}$ C NMR (101 MHz, Chloroform- $d$ )

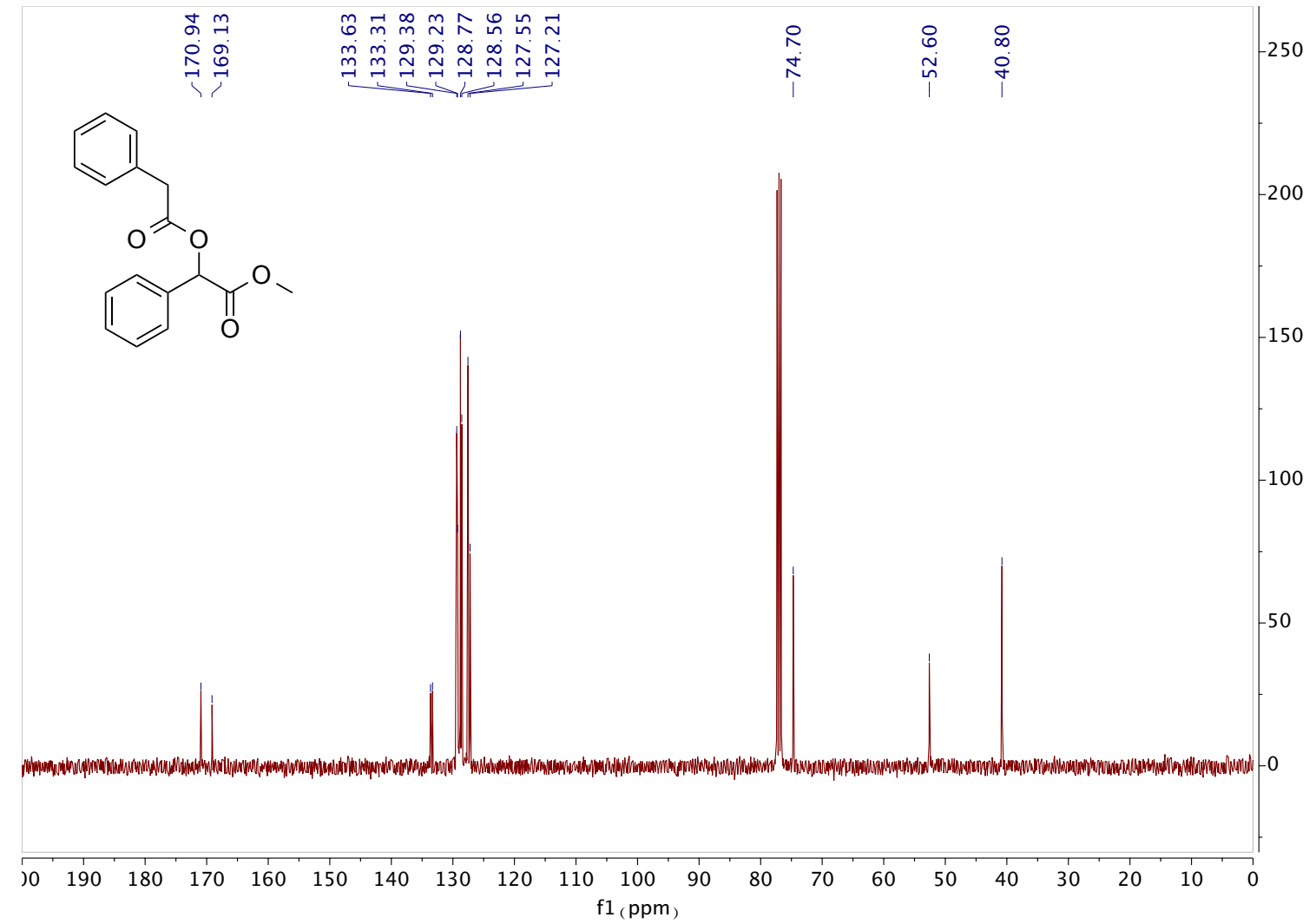


${ }^{1}$ H NMR (400 MHz, Chloroform- $d$ )

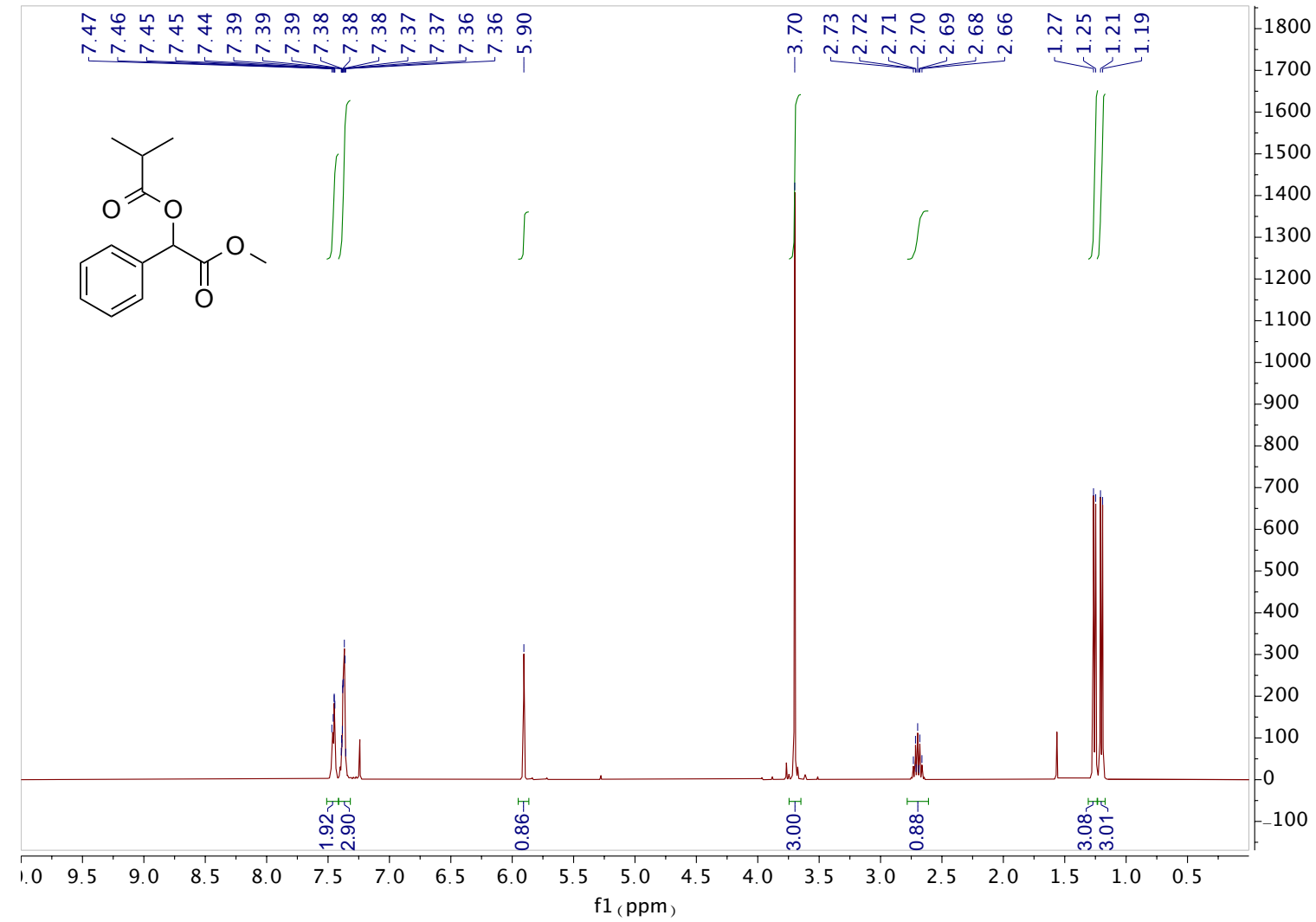

${ }^{13}$ C NMR (101 MHz, Chloroform- $d$ )

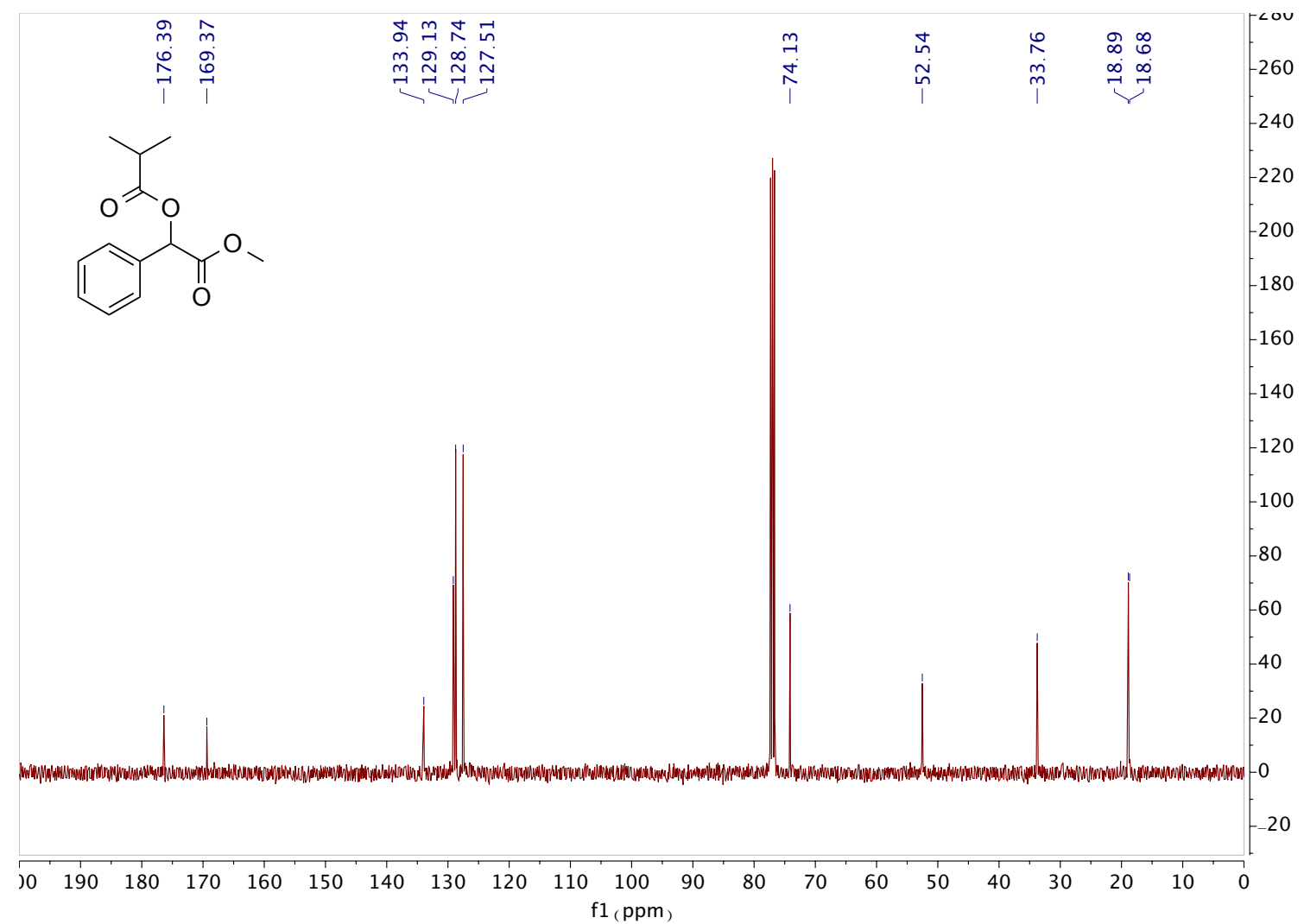


2-Methoxy-2-oxo-1-phenylethyl pivalate (12g)

${ }^{1}$ H NMR (400 MHz, Chloroform- $d$ )

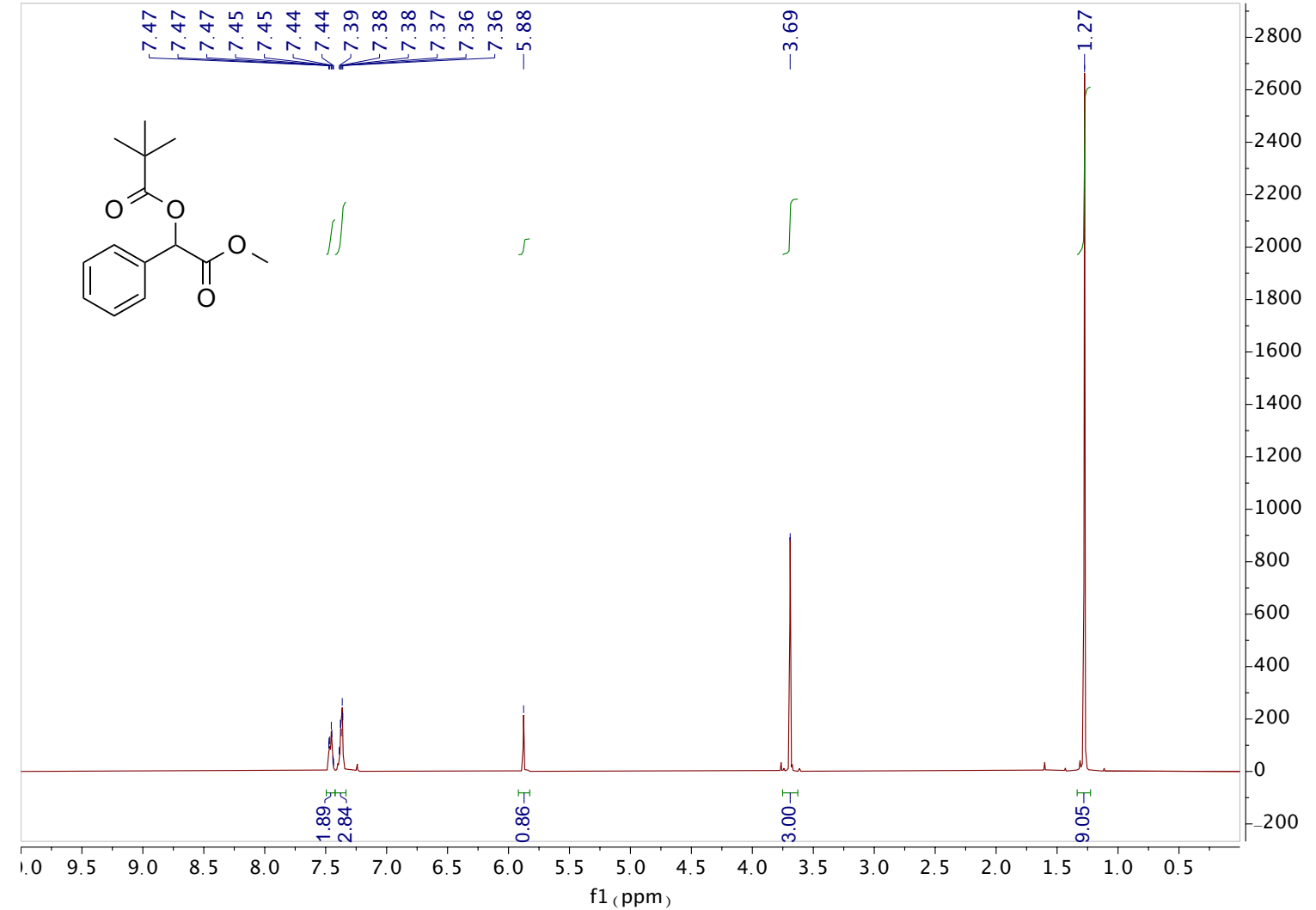

${ }^{13}$ C NMR (101 MHz, Chloroform- $d$ )

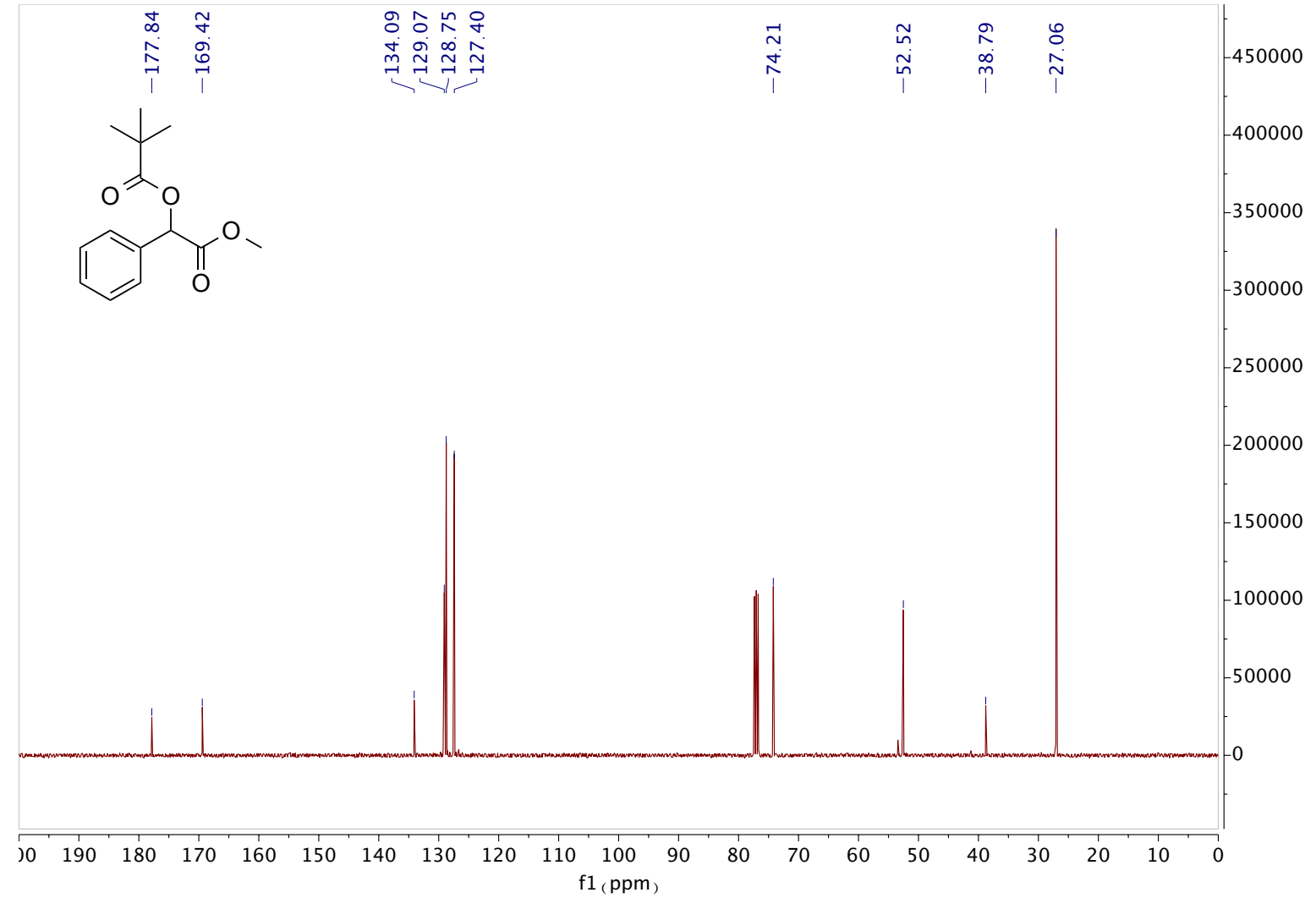


${ }^{1}$ H NMR (400 MHz, Chloroform- $d$ )

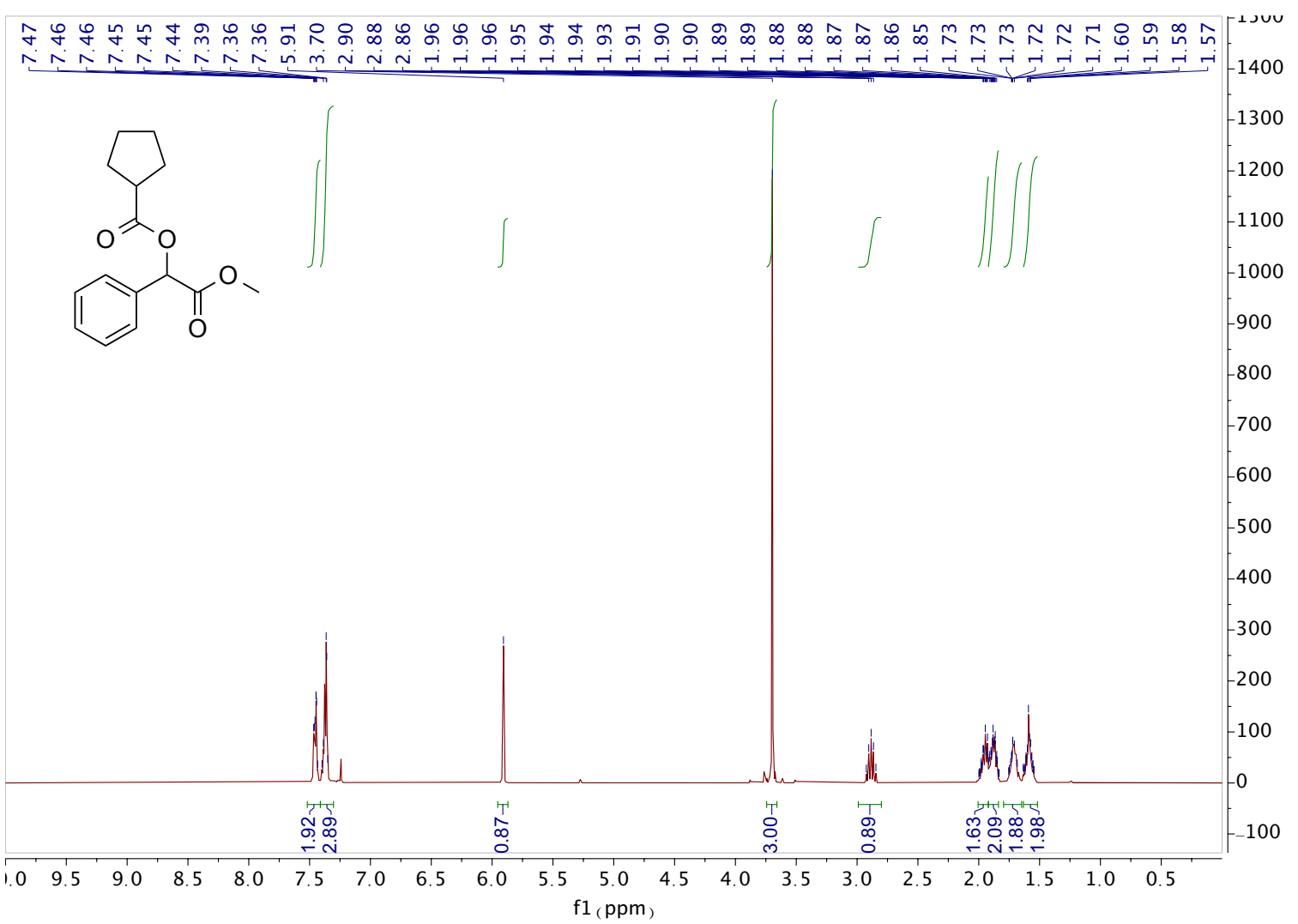

${ }^{13}$ C NMR (101 MHz, Chloroform- $d$ )

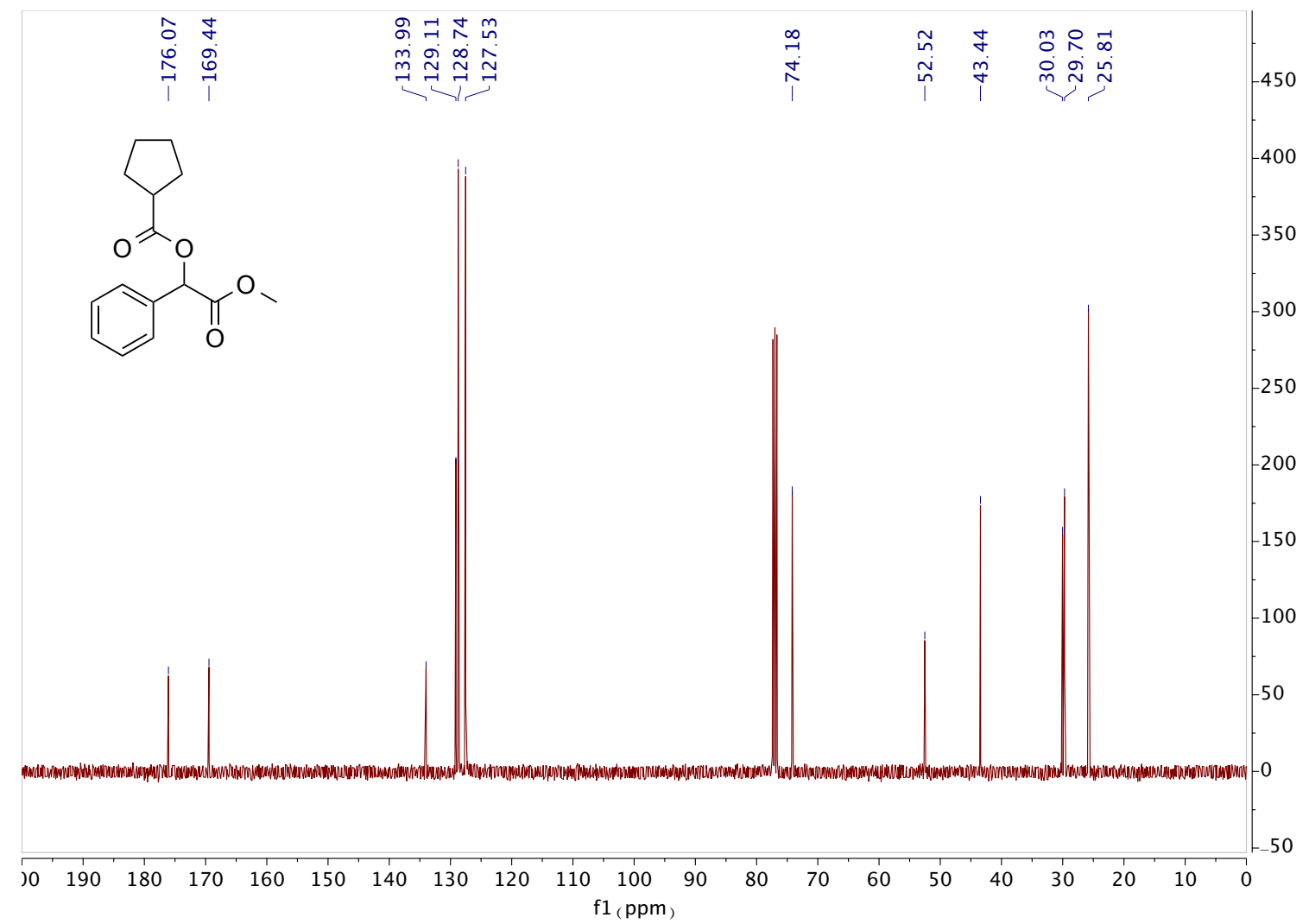


${ }^{1}$ H NMR (400 MHz, Chloroform- $d$ )

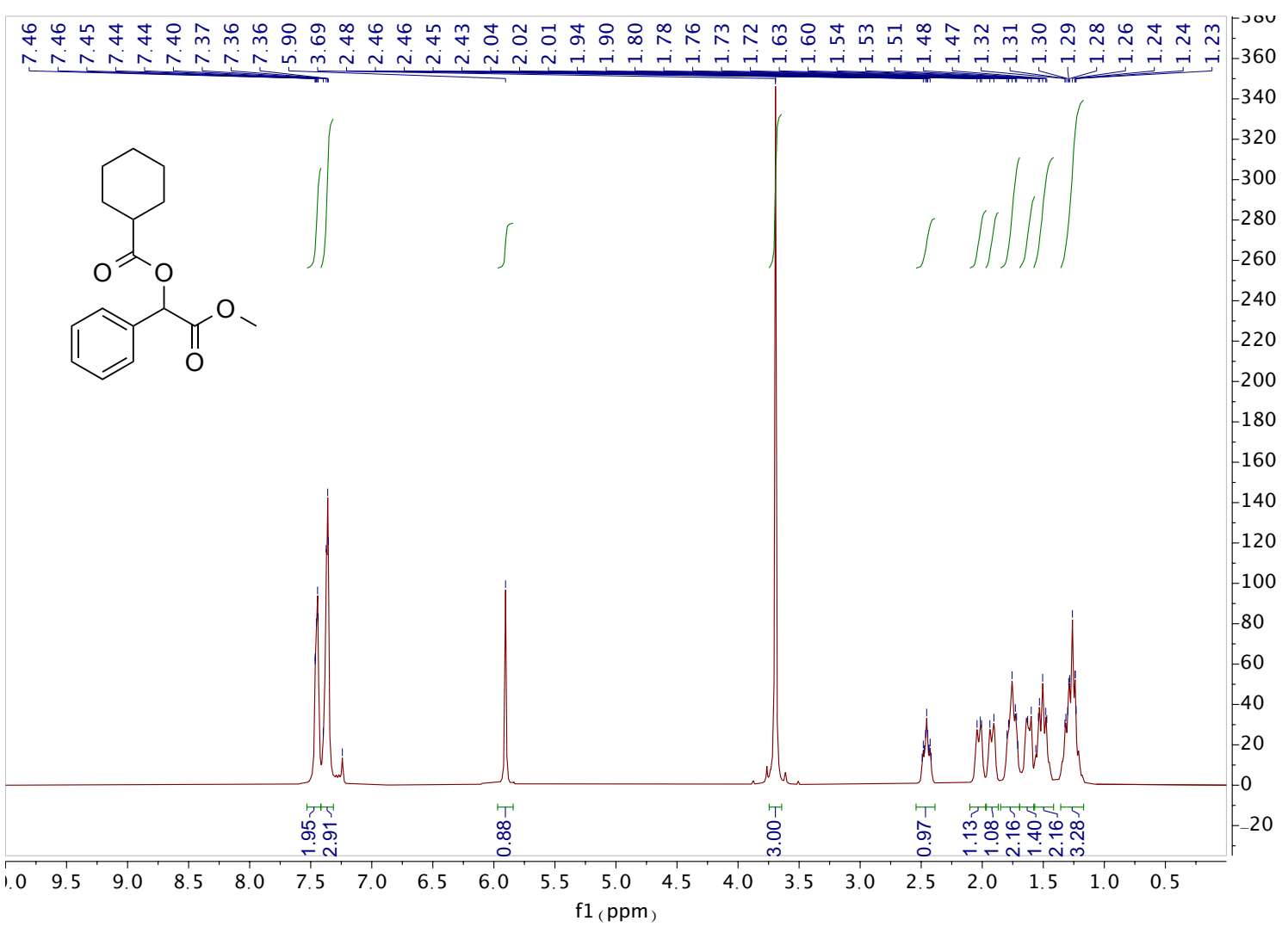

${ }^{13}$ C NMR (101 MHz, Chloroform- $d$ )

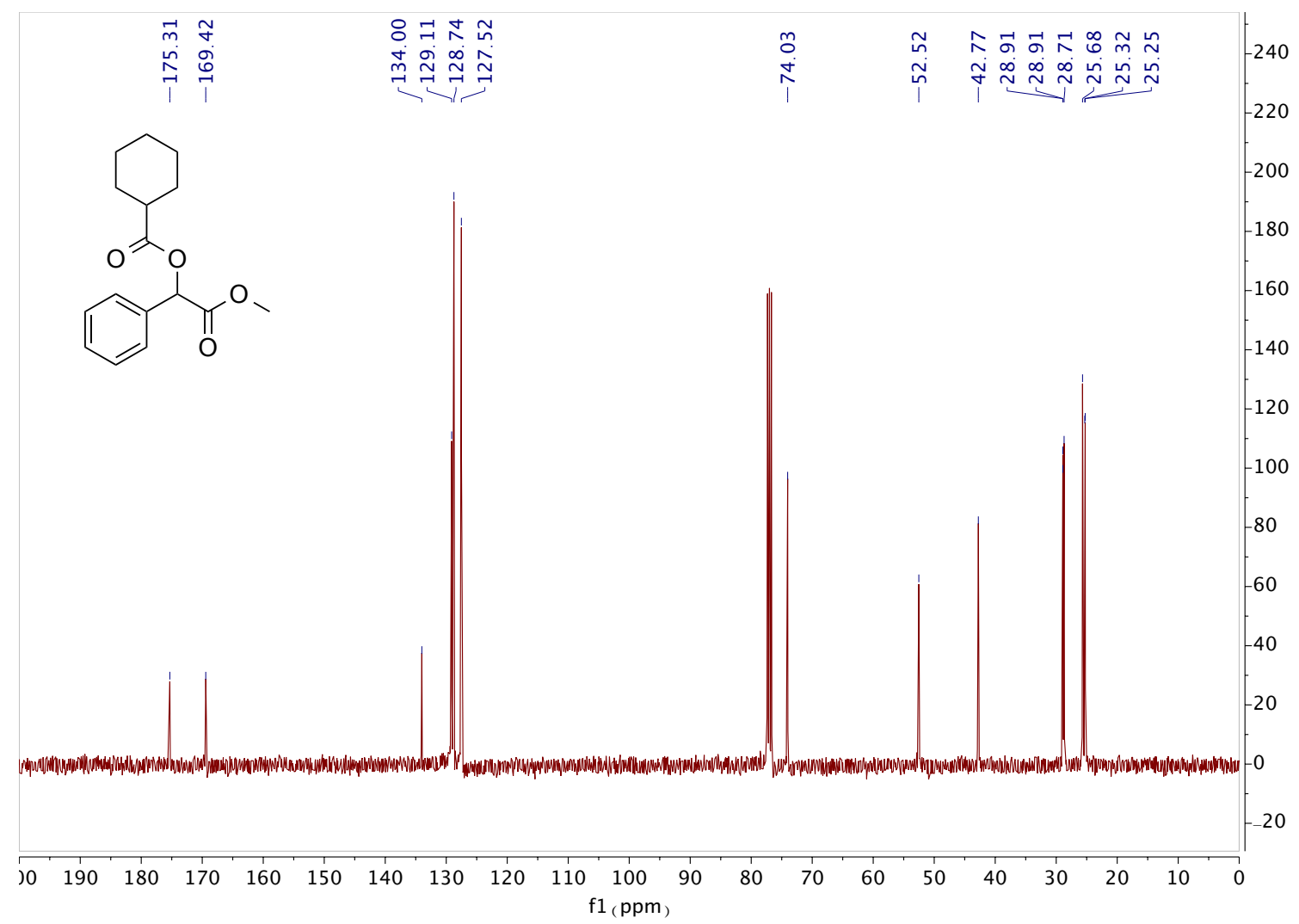


${ }^{1}$ H NMR (400 MHz, Chloroform- $d$ )

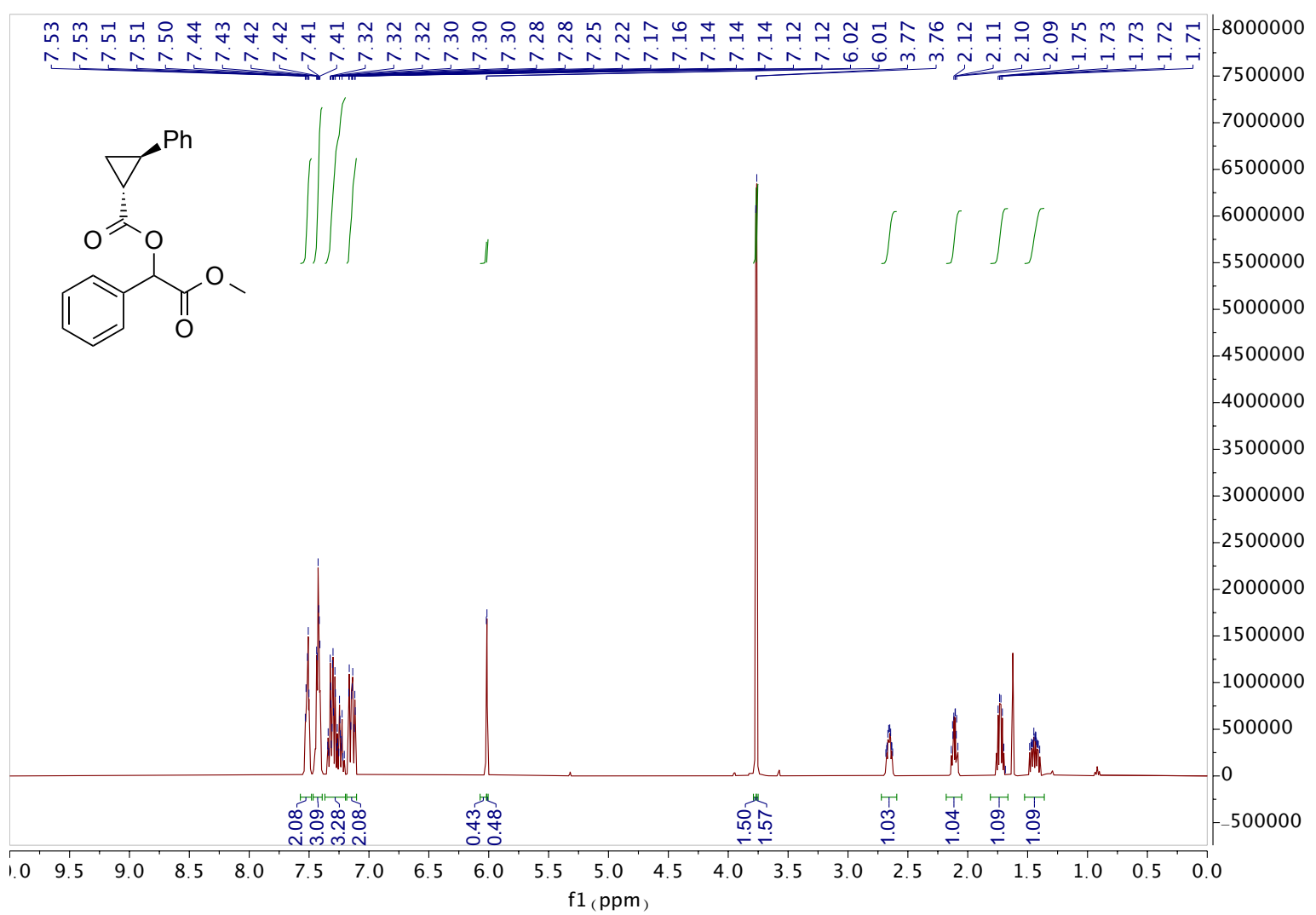

${ }^{13}$ C NMR (101 MHz, Chloroform- $d$ )

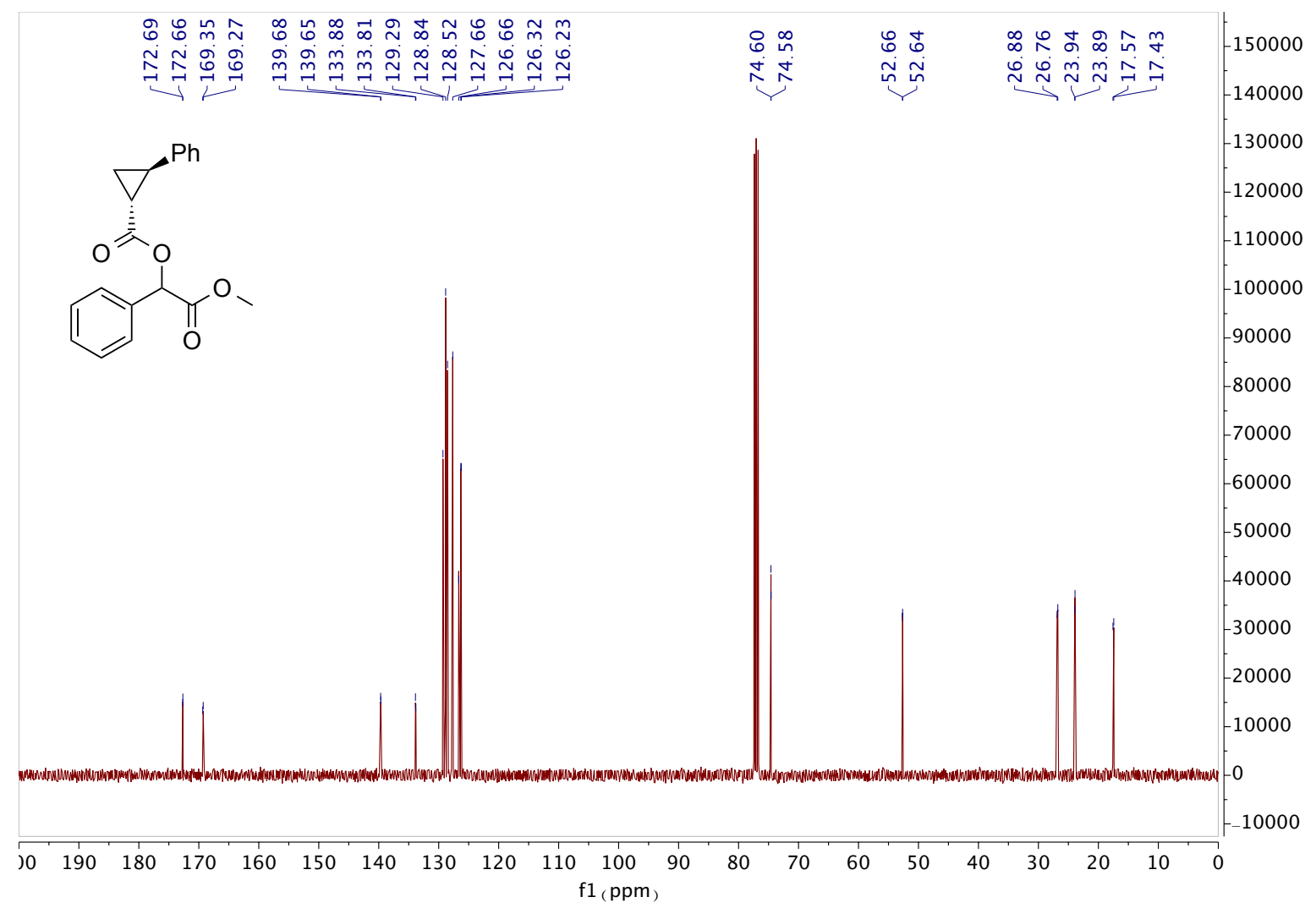




\section{2-Methoxy-2-oxo-1-phenylethyl 5-(1,2-dithiolan-3-yl)pentanoate (12k)}

\section{${ }^{1}$ H NMR (400 MHz, Chloroform- $d$ )}

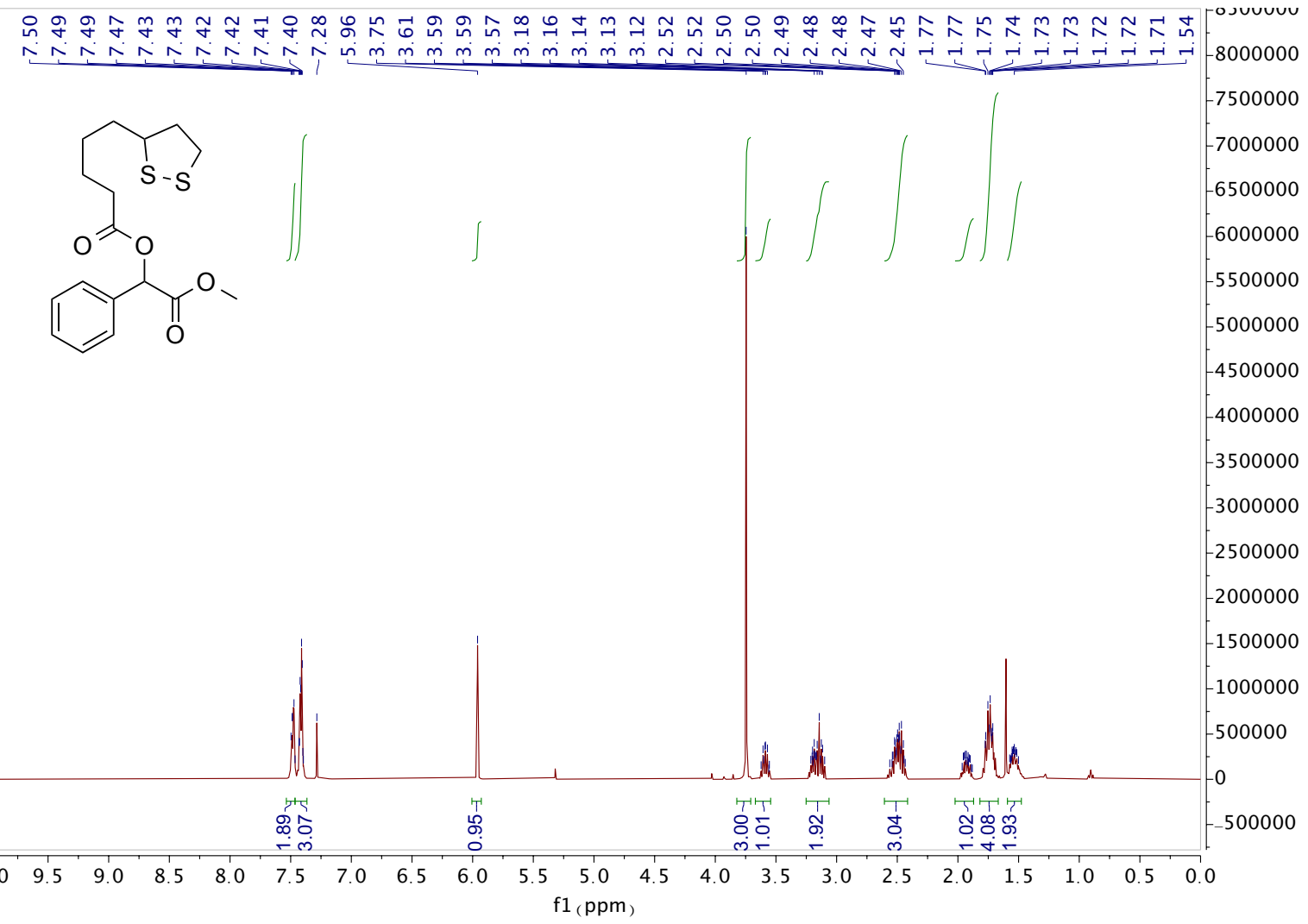

${ }^{13}$ C NMR (101 MHz, Chloroform- $d$ )

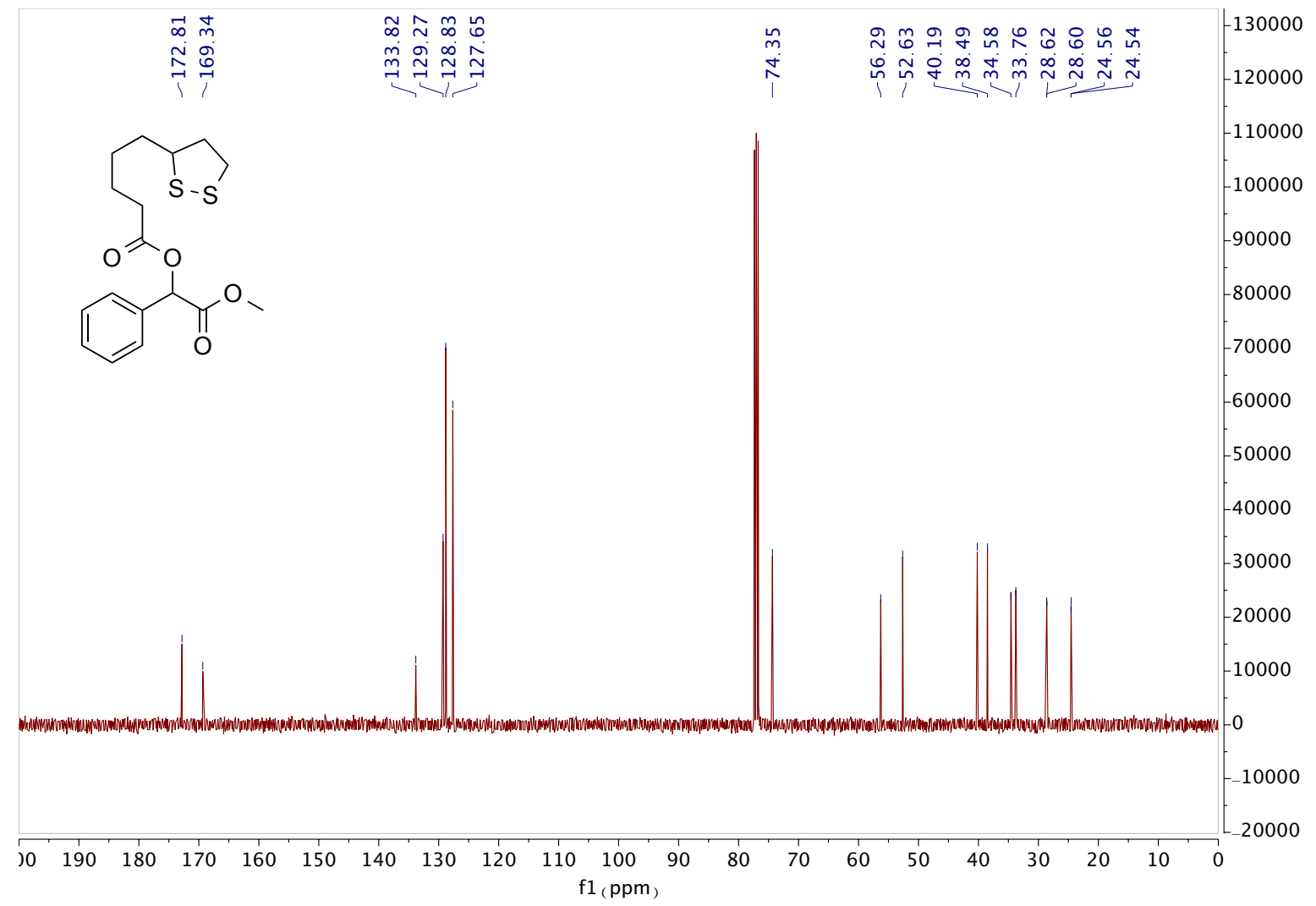


${ }^{1}$ H NMR (400 MHz, Chloroform- $d$ )

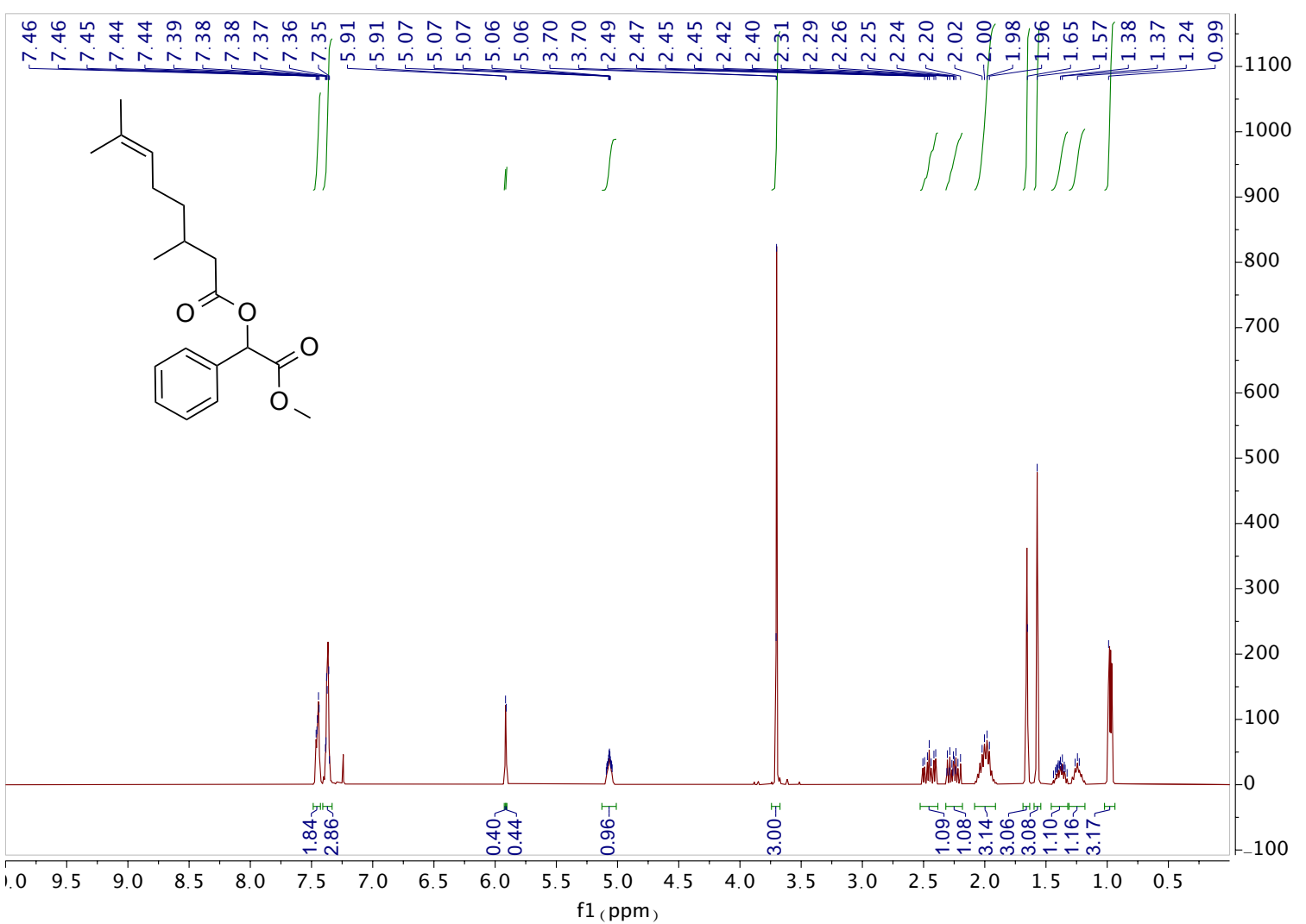

${ }^{13}$ C NMR (101 MHz, Chloroform- $d$ )

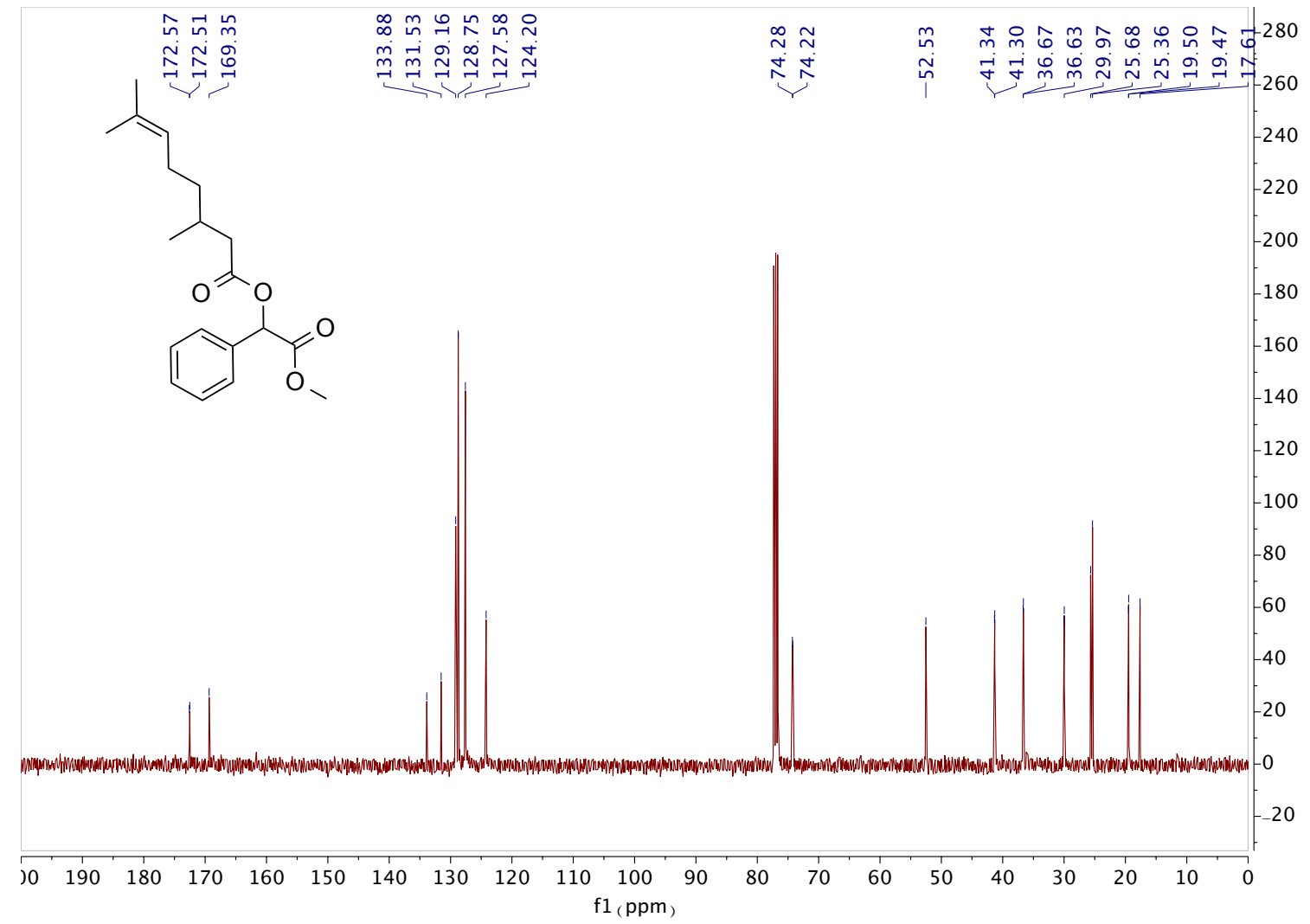

